\title{
Switching from Biaryl Formation to Amidation with Convoluted Polymeric Nickel Catalysis
}

\author{
Abhijit Sen, Raghu N. Dhital, Takuma Sato, Aya Ohno, Yoichi M. A. Yamada* \\ RIKEN, Center for Sustainable Resource Science, Wako, Saitama 351-0198, Japan \\ ymayamada@ riken.jp
}

\section{Contents}

1. General S2

2. Preparation of Catalyst S2

XAFS Analysis $\quad$ S3

Elemental Analysis $\quad$ S5

3. Optimization of Reaction Conditions S5

4. Catalysis

General Procedure for C-N Coupling S6

Characterization of C-N Coupling Products $\quad$ S6

General Procedure for C-C Coupling $\quad$ S10

Characterization of C-C Coupling Products $\quad$ S10

5. Intermolecular Amidation $\quad \mathrm{S} 12$

6. Synthesis of Biologically Active Molecules S13

7. Paracetamol Synthesis S13

8. Natural Product Synthesis S13

9. Large Scale Reaction $\quad$ S14

10. Reaction Mechanism

Identification of Intermediates $\quad \mathrm{S} 14$

Reaction Mechanism for C-N Coupling Reaction S18

11. Examples of Failed Attempts for C-C Coupling S19

12. Chemical Kinetics for C-N Coupling $\quad$ S19

13. Chemical Kinetics for C-C Coupling S25

14. Benzamide Addition on C-C Coupling S30

15. Phenylboronic Ester Addition on C-N Coupling S32

16. Reaction Time Course Measurements $\quad$ S34

17. Catalyst Recovery and Application S36

18. Generation of Ni(I) Catalyst S38

19. ICP-MS Analysis $\quad$ S38

20. References $\quad$ S39 


\section{General}

All chemicals and solvents were used as received without further purification unless otherwise mentioned. All the glasses (glassware) were cleaned by conc. $\mathrm{HCl}$ and rinsed by Milli-Q (18.2 M $\Omega$ ) water before setting up the reactions. ${ }^{1} \mathrm{H}-$ NMR (500 MHz), and ${ }^{13} \mathrm{C}-\mathrm{NMR}(125 \mathrm{MHz})$ spectra were measured with a JEOL JNM ECA-500 spectrometer at room temperature. Chemical shifts $(\delta)$ are expressed relative to the resonances of the residual non-deuterated solvent for ${ }^{1} \mathrm{H}\left[\mathrm{CDCl}_{3}\right]:{ }^{1} \mathrm{H}(\delta)=7.26 \mathrm{ppm}$, acetone- $\left.\mathrm{d}_{6}:{ }^{1} \mathrm{H}(\delta)=2.05 \mathrm{ppm}\right]$ and ${ }^{13} \mathrm{C}\left[\mathrm{CDCl}_{3}:{ }^{13} \mathrm{C}(\delta)=78.0 \mathrm{ppm}\right.$, acetone- $\mathrm{d}_{6}:{ }^{13} \mathrm{C}=29.8$ and $206.3 \mathrm{ppm}$ ]. Absolute values of the coupling constants are given in Hertz (Hz), regardless of their sign. Multiplicities are abbreviated as singlet (s), doublet (d), doublet of doublets (dd), triplet (t), quartet (q), quintet (quint), multiplet (m), and broad (br). Inductively coupled plasma mass spectrometry (ICP-MS) measurement was performed on a Perkin Elmer Nexion 300D. All the chemicals were purchased from commercial sources and used as received. Carbon-nitrogen and carbon-carbon coupling reactions were carried out using an organic synthesizer, ChemiStation (EYELA, PPS-1511).

TLC analysis was performed on Merck silica gel 60 F254. Flash column chromatography was carried out using Wakogel silica C-200 (particle size: 75-150 $\mu \mathrm{m}$ ). A series of combinations of ethyl acetate and hexane was used as an eluent. The GC was measured by an Agilent 6850 equipped with a capillary column (HP-1, column length 30 m, inner diameter 0.32 $\mathrm{mm}$ and film thickness $0.25 \mu \mathrm{m}$ or SLBTM-IL-60, column length $30 \mathrm{~m}$, inner diameter $0.25 \mathrm{~mm}$ and film thickness 0.20 $\mu \mathrm{m}$ or DB-Wax, column length $30 \mathrm{~m}$, inner diameter $0.25 \mathrm{~mm}$ and film thickness $0.25 \mu \mathrm{m})$. The GC-MS was measured by Agilent 7860A/JEOL JMS-T100GC equipped with a capillary column (HP-1, column length $30 \mathrm{~m}$, inner diameter 0.32 $\mathrm{mm}$ and film thickness $0.25 \mu \mathrm{m}$ or SLBTM-IL-60, column length $30 \mathrm{~m}$, inner diameter $0.25 \mathrm{~mm}$ and film thickness 0.20 $\mu \mathrm{m})$. ESI-MS mass was determined by using micrOTOFQIII instrument. Substrates $\mathbf{2} \mathbf{g}^{1}, \mathbf{2} \mathbf{h}^{2}$ and $\mathbf{2} \mathbf{i}^{2}$ were prepared following the literature report.

X-ray absorption fine structure (XAFS) experiment for Ni K-edge $(8.33 \mathrm{keV})$ was performed at BL14B2 beamline in SPring-8 (Japan). All the spectra were measured in transmission mode using ion chambers and Si (111) monochromator. The XAFS data processing was performed using Demeter software package ${ }^{3}$ with FEFF6 code. ${ }^{4}$ Density functional theory (DFT) calculation was carried out using ORCA program package, ${ }^{5}$ BP86 functional and Grimme's D3BJ dispersion correction $^{6,7}$ with def2-TZVP basis set were used.

\subsection{Preparation of Poly(4-vinylpyridine) Supported Nickel Catalyst}

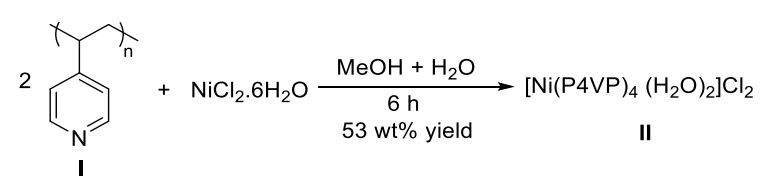

Procedure: Poly(4-vinylpyridine), P4VP (average $\mathrm{M}_{\mathrm{w}}=160000$, Aldrich, $210 \mathrm{mg}$ ) was dissolved in $20 \mathrm{~mL}$ of methanol. An aqueous solution $(20 \mathrm{~mL})$ of $\mathrm{NiCl}_{2} \cdot 6 \mathrm{H}_{2} \mathrm{O}(238 \mathrm{mg})$ was slowly added to the methanol solution. A greenish precipitate was observed during slow addition. The reaction mixture was stirred for more $6 \mathrm{~h}$ at room temperature. After that, the precipitate was filtered and the filtrate was discarded. The residue was washed three times with water $(3 \times 10 \mathrm{~mL})$, methanol $(4 \times 10 \mathrm{~mL})$ and diethyl ether $(5 \times 5 \mathrm{~mL})$. Next, the residue was collected and dried over vacuum for overnight (15 h) to get polymer supported nickel complex with $53 \mathrm{wt} \%$ yield $(233 \mathrm{mg})$.

\subsection{XAFS Analysis}


We proposed that the local structure of $\mathrm{P} 4 \mathrm{VP}-\mathrm{NiCl}_{2}$ is predominantly an ion pair of $\left[\mathrm{NiL}_{4}\left(\mathrm{OH}_{2}\right)_{2}\right]^{2+}(\mathrm{L}=$ pyridine unit in poly(4-vinylpyridine)) and $\mathrm{Cl}^{-}$based on XAFS data and elemental analysis which indicates that the molar ratio of $\mathrm{Ni}: \mathrm{Cl}: \mathrm{N}$ is ca. 1:2:6. Thus, Ni K-edge X-ray absorption near edge structure (XANES) spectra of $\mathrm{P} 4 \mathrm{VP}-\mathrm{NiCl}_{2}$ and reference materials ( $\mathrm{Ni}$ foil, $\mathrm{NiO}$, anhydrous $\mathrm{NiCl}_{2}$, and hydrated $\mathrm{Ni}$ (II) salts) indicate that the oxidation number of the as-prepared catalyst is +2 (Figure S1), and extended X-ray absorption fine structure (EXAFS) suggests that there is no Ni-Cl bond. Next, the optimized structure of $\left[\mathrm{Ni}(\text { pyridine })_{4}\left(\mathrm{OH}_{2}\right)_{2}\right]^{2+}$ was obtained by DFT method (Figure S2), and then the fitting of EXAFS was carried out using the DFT-calculated geometry as a local structure model (Figure S3). The fitting parameters show good agreement ( $\mathrm{R}$ factor of 0.023) with the proposed model, although the other geometrical isomer and the partial substitution of the polymeric ligand with water molecules are also possible due to the accuracy limitation of EXAFS. At least, it is quite clear that there is no $\mathrm{Ni}-\mathrm{Cl}$ bond as indicated in EXAFS. In any case, the above result implies that the $\mathrm{Ni}^{2+}$ center is coordinated by the polymer and water, and the $\mathrm{Cl}^{-}$anions are dissociated from the $\mathrm{Ni}^{2+}$ center to form an ion pair.

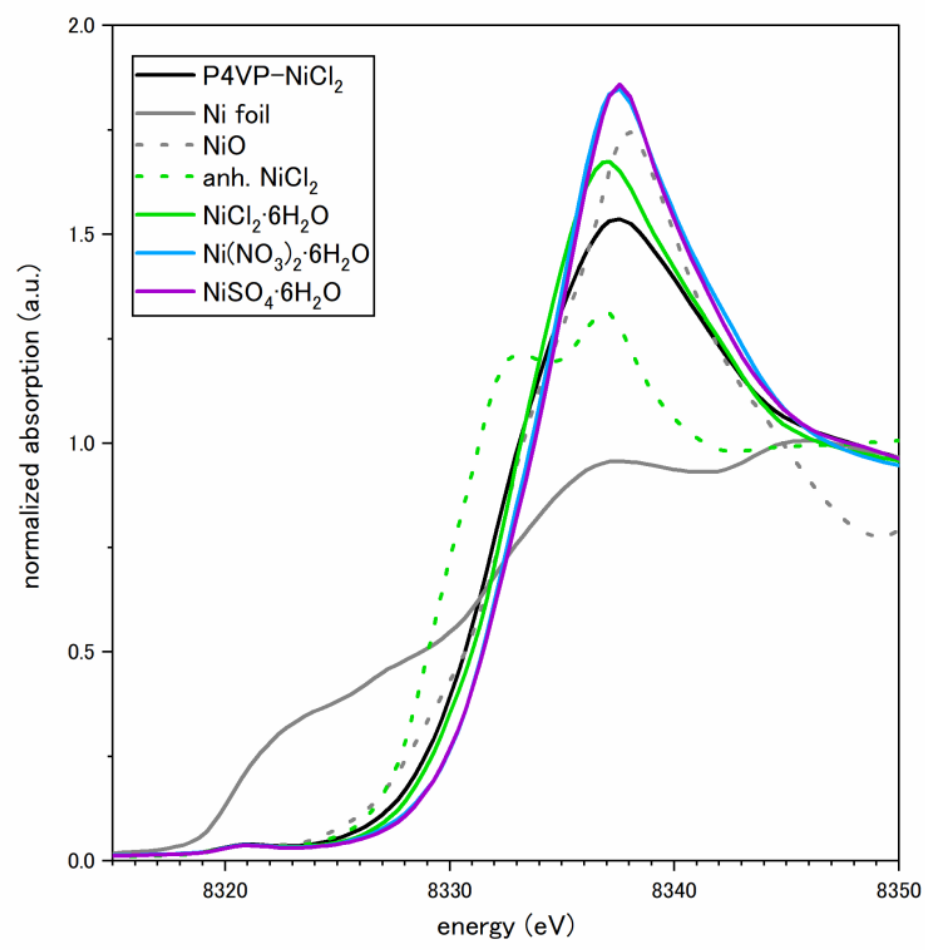

Figure S1. Ni K-edge XANES spectra of P4VP-Ni and reference materials. 


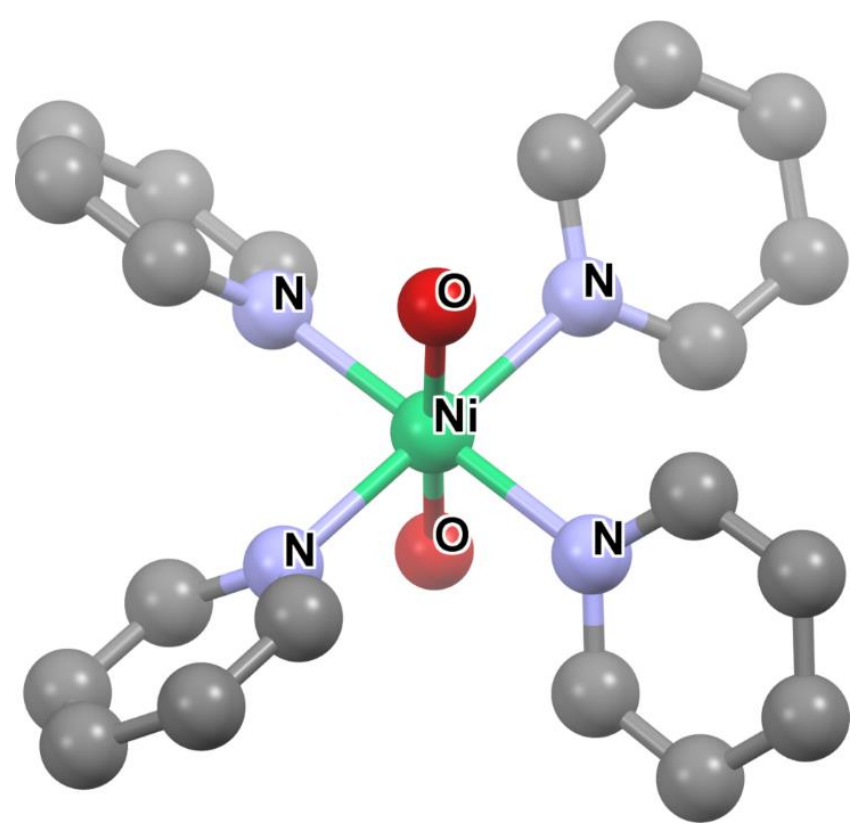

Figure S2. [Ni(pyridine) $\left.)_{4}\left(\mathrm{OH}_{2}\right)_{2}\right]^{2+}$ as a local structure model for EXAFS analysis (UBP86-D3BJ/def2TZVP). Hydrogen atoms are omitted for clarity. Average Ni-N distance: $2.10 \AA$, average Ni-O distance: $2.19 \AA$.

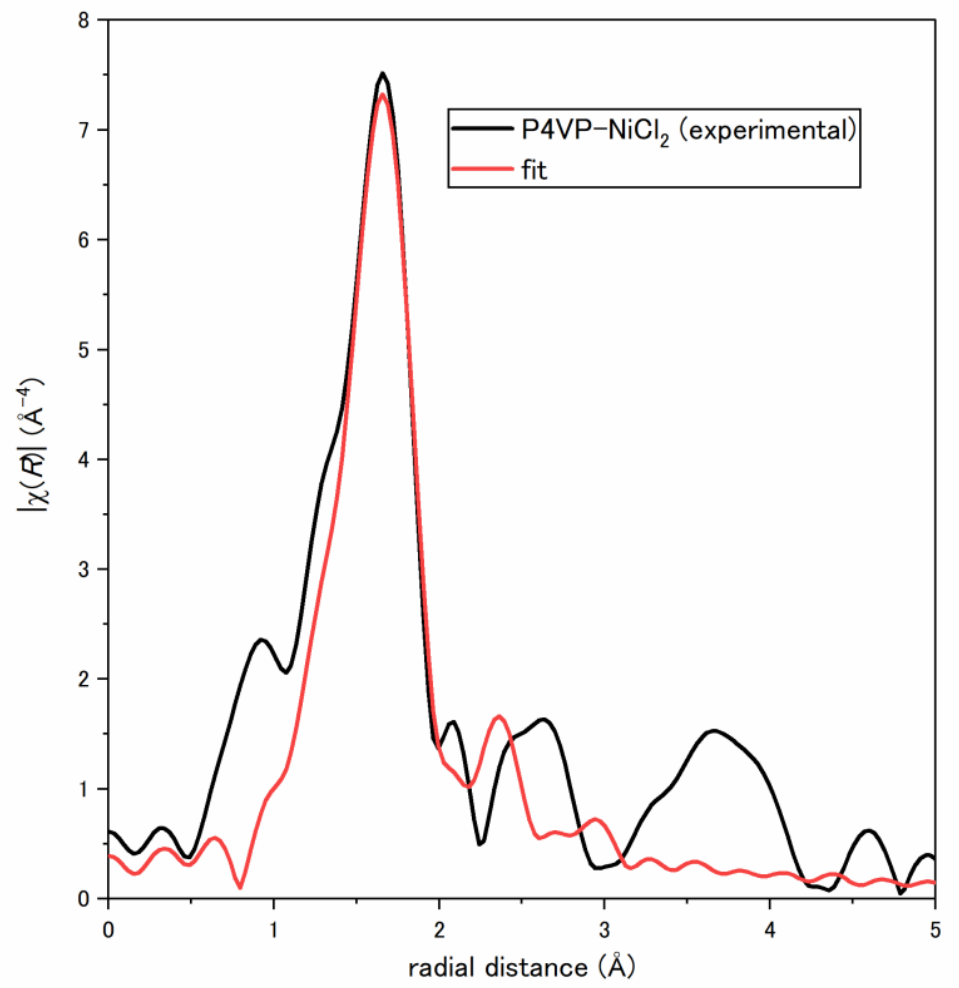




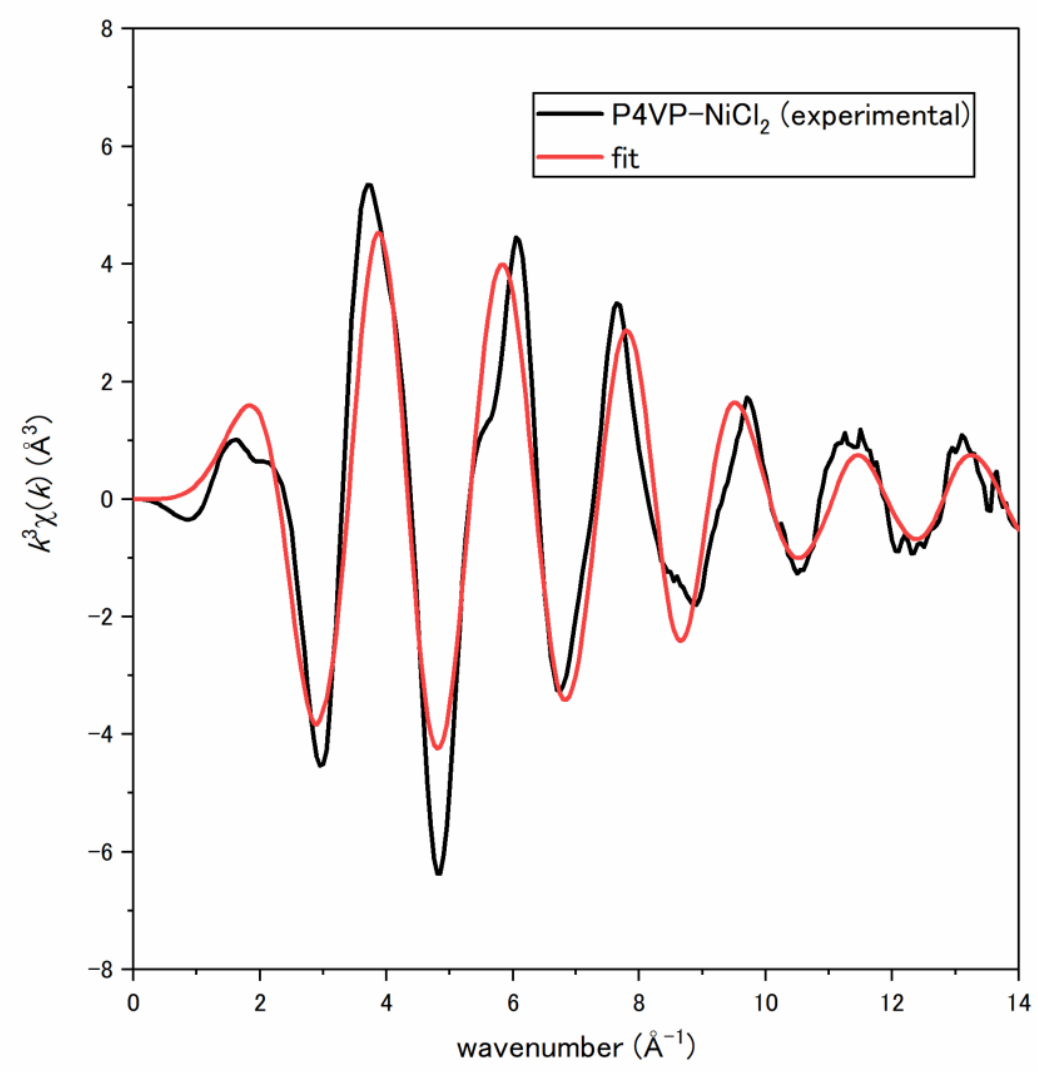

Figure S3. Ni K-edge $k^{3}$-weighted EXAFS of P4VP-Ni and the fit based on the DFT-based model structure (R factor of 0.023, $k$-range: $3-14 \AA^{-1}, R$-range: $1.1-2.1 \AA$ ). $\Delta E=-0.2 \mathrm{eV}, \Delta R \_\mathrm{N}=-0.025 \AA$, $\Delta R_{-} \mathrm{O}=-0.007 \AA, \sigma_{-}^{2} \mathrm{~N}=0.00357 \AA^{2}, \sigma_{-}^{2} \mathrm{O}=0.00318 \AA^{2}$.

\subsection{Elemental Analysis:}

Nickel: 6.94\%, Chloride: 8.21\%, Carbon: 60.62\%, Hydrogen: 6.05\% and Nitrogen: 10.09\%.

\section{Optimization of Reaction Conditions ${ }^{a}$}

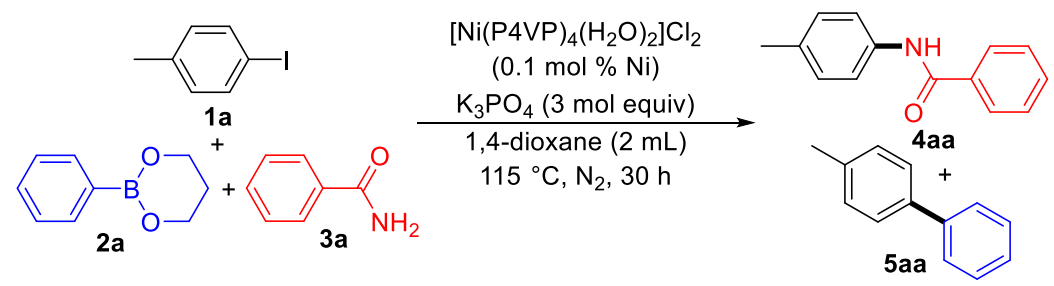

$\begin{array}{cccr}\text { entry } & \text { deviation from standard conditions } & \text { yield } \mathbf{4 a a}(\%) & \text { yield } \mathbf{5 a a}(\%) \\ 1 & \text { none } & 99 & 0 \\ 2 & \text { phenylboronic acid instead of } 2 \mathbf{a} & 98 & 1 \\ 3 & \text { without } 2 \mathbf{a} & 0 & 0 \\ 4 & \text { without }\left[\mathrm{Ni}(\mathrm{P} 4 \mathrm{VP})_{4}\left(\mathrm{H}_{2} \mathrm{O}\right)_{2}\right] \mathrm{Cl}_{2} & 0 & 0 \\ 5 & \mathrm{NiCl}_{2} \cdot 6 \mathrm{H}_{2} \mathrm{O} \text { instead of }\left[\mathrm{Ni}(\mathrm{P} 4 \mathrm{VP})_{4}\left(\mathrm{H}_{2} \mathrm{O}\right)_{2}\right] \mathrm{Cl}_{2} & 82 & 2 \\ 6 & \mathrm{NiCl}_{2}(\text { anhydrous }) \text { instead of }\left[\mathrm{Ni}(\mathrm{P} 4 \mathrm{VP})_{4}\left(\mathrm{H}_{2} \mathrm{O}\right)_{2}\right] \mathrm{Cl}_{2} & 83 & 1 \\ 7 & \mathrm{PdCl}_{2} \text { instead of }\left[\mathrm{Ni}(\mathrm{P} 4 \mathrm{VP})_{4}\left(\mathrm{H}_{2} \mathrm{O}\right)_{2}\right] \mathrm{Cl}_{2} & 0 & 56\end{array}$




\begin{tabular}{|c|c|c|c|}
\hline 8 & without $\mathrm{K}_{3} \mathrm{PO}_{4}$ & 0 & 0 \\
\hline 9 & $\mathrm{Na}_{3} \mathrm{PO}_{4}$ instead of $\mathrm{K}_{3} \mathrm{PO}_{4}$ & 12 & 9 \\
\hline 10 & $\mathrm{Li}_{3} \mathrm{PO}_{4}$ instead of $\mathrm{K}_{3} \mathrm{PO}_{4}$ & 0 & 6 \\
\hline 11 & $\mathrm{KO} t \mathrm{Bu}$ instead of $\mathrm{K}_{3} \mathrm{PO}_{4}$ & 97 & 2 \\
\hline 12 & $\mathrm{NaO} t \mathrm{Bu}$ instead of $\mathrm{K}_{3} \mathrm{PO}_{4}$ & 55 & 33 \\
\hline 13 & toluene instead of 1,4-dioxane & 60 & 0 \\
\hline 14 & without $\mathbf{3 a}$ & 0 & 55 \\
\hline 15 & without $3 \mathbf{a}$ and 3 equiv of $\mathbf{2 a}$ & 0 & 78 \\
\hline 16 & without $3 \mathbf{a}$ and 5 equiv of $\mathbf{2 a}$ & 0 & 91 \\
\hline 17 & air instead of nitrogen & 94 & 0 \\
\hline 18 & $90^{\circ} \mathrm{C}$ instead of $115^{\circ} \mathrm{C}$ & 65 & 0 \\
\hline $19^{b}$ & {$\left[\mathrm{Ni}(\mathrm{P} 4 \mathrm{VP})_{4}\left(\mathrm{H}_{2} \mathrm{O}\right)_{2}\right] \mathrm{Cl}_{2}(0.05 \mathrm{~mol} \% \mathrm{Ni}$ instead of $0.1 \mathrm{~mol} \% \mathrm{Ni})$} & 46 & 0 \\
\hline 20 & $24 \mathrm{~h}$ instead of $30 \mathrm{~h}$ & 94 & 0 \\
\hline 21 & $p$-tolyl triflate instead of $\mathbf{1 a}$ & trace & 0 \\
\hline 22 & $p$-tolyl tosylate instead of $\mathbf{1 a}$ & trace & 0 \\
\hline
\end{tabular}

${ }^{a}$ All reactions were performed with $\mathbf{1 a}$ ( 1 mol equiv, $0.5 \mathrm{mmol}$ ), $\mathbf{2 a}$ ( $1.5 \mathrm{~mol}$ equiv), 3a ( 3 mol equiv), nickel catalyst $(0.1 \mathrm{~mol} \% \mathrm{Ni}), \mathrm{K}_{3} \mathrm{PO}_{4}$ (3 mol equiv) and 1,4-dioxane $(2 \mathrm{~mL})$ at $115{ }^{\circ} \mathrm{C}$ for $30 \mathrm{~h}$ under $\mathrm{N}_{2} .{ }^{b}$ Reaction was performed in 1 mmol scale

\section{Catalysis}

\subsection{General Procedure for Amidation Reaction}

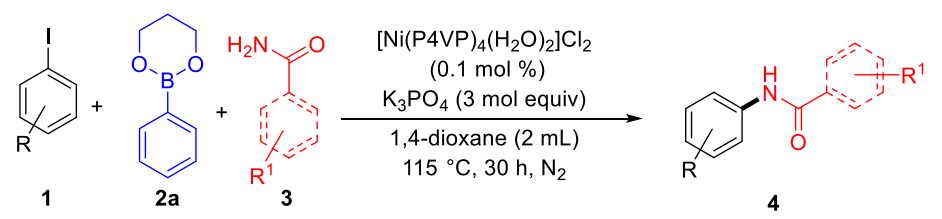

A mixture of $\left[\mathrm{Ni}(\mathrm{P} 4 \mathrm{VP})_{4}\left(\mathrm{H}_{2} \mathrm{O}\right)_{2}\right] \mathrm{Cl}_{2}(1000 \mathrm{~mol} \mathrm{ppm}, 0.42 \mathrm{mg})$, aryl iodide, 1 (1 mol equiv, 0.5 mmol), phenylboronic ester, 2a (1.5 mol equiv, $0.75 \mathrm{mmol}, 243 \mathrm{mg}$ ), amide substrates 3 ( $3 \mathrm{~mol}$ equiv, $1.5 \mathrm{mmol}$ ) and $\mathrm{K}_{3} \mathrm{PO}_{4}(3 \mathrm{~mol}$ equiv, 1.5 mmol, $318 \mathrm{mg}$ ) were added to a reaction tube. The reaction tube was degassed under vacuum and refile with $\mathrm{N}_{2}$ under standard Schlack techniques (3 times). 1,4-dioxane (2 mL) was added and seal the reaction tube with teflon, followed by stirring at $115{ }^{\circ} \mathrm{C}$ (chemiStation) under nitrogen for $30 \mathrm{~h}$. After that the reaction mixture was diluted by EtOAc and extracted with $\mathrm{H}_{2} \mathrm{O}$. The organic layer was collected and dried over $\mathrm{Na}_{2} \mathrm{SO}_{4}$ and filtered. The solvent was evaporated under vacuum and crude mass was purified by column chromatography (hexane/EtOAc $=5 / 1$ ) to give products 4 .

\subsection{Characterization of Products}

$N$-(p-tolyl)butyramide (4ai, Ref. 8)

White solid $(98 \%$ yield, $86 \mathrm{mg}) \cdot \mathrm{m} . \mathrm{p} .72{ }^{\circ} \mathrm{C}-73{ }^{\circ} \mathrm{C} ;{ }^{1} \mathrm{H}-\mathrm{NMR}\left(500 \mathrm{MHz}, \mathrm{CDCl}_{3}\right) \delta 7.39(\mathrm{~d}, J=7.8 \mathrm{~Hz}$,
$2 \mathrm{H}), 7.26(\mathrm{~s}, 1 \mathrm{H}), 7.10(\mathrm{~d}, J=7.8 \mathrm{~Hz}, 2 \mathrm{H}), 2.20-2.45(\mathrm{~m}, 5 \mathrm{H}), 1.72-1.77(\mathrm{~m}, 2 \mathrm{H}), 0.99(\mathrm{t}, J=7.2 \mathrm{~Hz}$, $3 \mathrm{H}) .{ }^{13} \mathrm{C}\left\{{ }^{1} \mathrm{H}\right\}-\mathrm{NMR}\left(125 \mathrm{MHz}, \mathrm{CDCl}_{3}\right) \delta 171.3,135.5,133.9,129.5,120.0,39.7,20.9,19.2,13.8$.

$N$-(p-tolyl)benzamide (4aa, Ref. 9) 
White solid (99\% yield, $104 \mathrm{mg})$. m.p. $148^{\circ} \mathrm{C}-149^{\circ} \mathrm{C} ;{ }^{1} \mathrm{H}-\mathrm{NMR}\left(500 \mathrm{MHz}, \mathrm{CDCl}_{3}\right) \delta 7.84-7.88(\mathrm{~m}, 3 \mathrm{H})$,

7.44-7.53 (m, 6H), 7.15-7.21 (m, 2H), $2.34(\mathrm{~s}, 3 \mathrm{H}) .{ }^{13} \mathrm{C}\left\{{ }^{1} \mathrm{H}\right\}-\mathrm{NMR}\left(125 \mathrm{MHz}, \mathrm{CDCl}_{3}\right) \delta 165.8,135.7$, $135.2,134.4,131.8,129.7,128.8,127.1,120.5,21.0$.

$N$-(p-tolyl)acetamide (4ah, Ref. 10)

White solid (82\% yield, $61 \mathrm{mg})$. m. p. $142^{\circ} \mathrm{C}-143{ }^{\circ} \mathrm{C} ;{ }^{1} \mathrm{H}-\mathrm{NMR}\left(500 \mathrm{MHz}, \mathrm{CDCl}_{3}\right) \delta 7.37(\mathrm{~d}, J=8.0 \mathrm{~Hz}$, $2 \mathrm{H}), 7.11(\mathrm{~d}, J=8.0 \mathrm{~Hz}, 2 \mathrm{H}), 2.30(\mathrm{~s}, 3 \mathrm{H}), 2.15(\mathrm{~s}, 3 \mathrm{H}) .{ }^{13} \mathrm{C}\left\{{ }^{1} \mathrm{H}\right\}-\mathrm{NMR}\left(125 \mathrm{MHz}, \mathrm{CDCl}_{3}\right) \delta 168.3,135.4$, 134.0, 129.5, 120.1, 24.6, 20.9.

$N$-(4-acetylphenyl)benzamide (4na, Ref. 11)

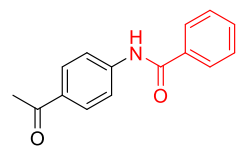

White solid (62\% yield, $74 \mathrm{mg})$. m. p. $203^{\circ} \mathrm{C}-204^{\circ} \mathrm{C} ;{ }^{1} \mathrm{H}-\mathrm{NMR}\left(500 \mathrm{MHz}, \mathrm{CDCl}_{3}\right) \delta 7.98-8.01(\mathrm{~m}$, $3 \mathrm{H})$, 7.87-7.89 (m, 2H), 7.76-7.78 (m, 1H), 7.50-7.56 (m, 3H), 7.25 (d, J = 2.9 Hz, 1H), 2.59 (s, 3H). ${ }^{13} \mathrm{C}\left\{{ }^{1} \mathrm{H}\right\}-\mathrm{NMR}\left(125 \mathrm{MHz}, \mathrm{CDCl}_{3}\right) \delta 197.0,165.8,142.4,134.6,133.3,132.4,129.9,129.0,127.2,119.3$,

26.5.

$N$-(3,5-dichlorophenyl)benzamide (4ka, Ref. 12)

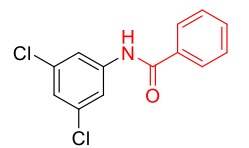

White solid $(82 \%, 109 \mathrm{mg})$. m. p. $149^{\circ} \mathrm{C}-150^{\circ} \mathrm{C} ;{ }^{1} \mathrm{H}-\mathrm{NMR}\left(500 \mathrm{MHz}, \mathrm{CDCl}_{3}\right) \delta 8.13(\mathrm{~s}, 1 \mathrm{H}), 7.81(\mathrm{~d}$, $J=7.4 \mathrm{~Hz}, 2 \mathrm{H}), 7.59(\mathrm{~d}, J=1.7 \mathrm{~Hz}, 2 \mathrm{H}), 7.53(\mathrm{t}, J=7.4 \mathrm{~Hz}, 1 \mathrm{H}), 7.43(\mathrm{t}, J=7.4 \mathrm{~Hz}, 2 \mathrm{H}), 7.10(\mathrm{t}, J=$ $1.7 \mathrm{~Hz}, 1 \mathrm{H}) .{ }^{13} \mathrm{C}\left\{{ }^{1} \mathrm{H}\right\}-\mathrm{NMR}\left(125 \mathrm{MHz}, \mathrm{CDCl}_{3}\right) \delta 166.14,139.79,135.36,134.14,132.43,128.96$,

127.17, 124.60, 118.68 .

3-methyl- $N$-(p-tolyl)benzamide (4ac, Ref. 13)

White solid (94\%, $106 \mathrm{mg})$. m.p. $110^{\circ} \mathrm{C}-111^{\circ} \mathrm{C} ;{ }^{1} \mathrm{H}-\mathrm{NMR}\left(500 \mathrm{MHz}, \mathrm{CDCl}_{3}\right) \delta 7.70-7.91(\mathrm{~m}, 1 \mathrm{H}), 7.62-$ $7.66(\mathrm{~m}, 2 \mathrm{H}), 7.51(\mathrm{~d}, J=7.8 \mathrm{~Hz}, 2 \mathrm{H}), 7.27-7.38(\mathrm{~m}, 2 \mathrm{H}), 7.15(\mathrm{~d}, J=7.8 \mathrm{~Hz}, 2 \mathrm{H}), 2.40$ (s, 3H), 2.33 (s, $3 \mathrm{H}) .{ }^{13} \mathrm{C}\left\{{ }^{1} \mathrm{H}\right\}$-NMR $\left(125 \mathrm{MHz}, \mathrm{CDCl}_{3}\right) \delta 166.0,138.7,135.5,135.2,134.2,132.5,129.7,128.7,127.8$, 124.0, 120.4, 21.5, 21.0.

$N$-(p-tolyl)nicotinamide (3ag, Ref. 9)

White solid $(68 \%, 72 \mathrm{mg})$. m.p. $144^{\circ} \mathrm{C}-145^{\circ} \mathrm{C} ;{ }^{1} \mathrm{H}-\mathrm{NMR}\left(500 \mathrm{MHz}, \mathrm{CDCl}_{3}\right) \delta 8.89-9.09(\mathrm{~m}, 1 \mathrm{H}), 8.64-$ $8.74(\mathrm{~m}, 1 \mathrm{H}), 8.34-8.47(\mathrm{~m}, 1 \mathrm{H}), 8.15$ (brs, $1 \mathrm{H}), 7.49(\mathrm{~d}, J=7.4 \mathrm{~Hz}, 2 \mathrm{H}), 7.28-7.42(\mathrm{~m}, 1 \mathrm{H}), 7.14(\mathrm{~d}, J=$ $7.4 \mathrm{~Hz}, 2 \mathrm{H}), 2.32(\mathrm{~s}, 3 \mathrm{H}) .{ }^{13} \mathrm{C}\left\{{ }^{1} \mathrm{H}\right\}-\mathrm{NMR}\left(125 \mathrm{MHz}, \mathrm{CDCl}_{3}\right) \delta 164.1,152.3,148.0,135.5,135.1,134.9$, 131.0, 129.7, 123.7, 120.8, 21.0.

$N$-(thiophen-3-yl)benzamide (4ta, Ref. 14)

White solid $(69 \%, 71 \mathrm{mg})$. m.p. $148^{\circ} \mathrm{C}-149^{\circ} \mathrm{C} ;{ }^{1} \mathrm{H}-\mathrm{NMR}\left(500 \mathrm{MHz}, \mathrm{CDCl}_{3}\right) \delta 8.12$ (brs, $\left.1 \mathrm{H}\right), 7.86(\mathrm{t}, J=$ $6.6 \mathrm{~Hz}, 2 \mathrm{H}), 7.71-7.74(\mathrm{~m}, 1 \mathrm{H}), 7.54(\mathrm{t}, J=6.6 \mathrm{~Hz}, 1 \mathrm{H}), 7.48(\mathrm{~d}, J=7.4 \mathrm{~Hz}, 2 \mathrm{H}), 7.25-7.29(\mathrm{~m}, 1 \mathrm{H}), 7.12$ $(\mathrm{d}, J=5.2 \mathrm{~Hz}, 1 \mathrm{H}) .{ }^{13} \mathrm{C}\left\{{ }^{1} \mathrm{H}\right\}-\mathrm{NMR}\left(125 \mathrm{MHz}, \mathrm{CDCl}_{3}\right) \delta 165.1,135.7,134.5,132.0,128.9,127.1,124.8$,

121.3, 110.9 .

$N$-(m-tolyl)benzamide (4ba, Ref 15)

White Solid (89\%, $94 \mathrm{mg})$. m.p. $124^{\circ} \mathrm{C}-125^{\circ} \mathrm{C} ;{ }^{1} \mathrm{H}-\mathrm{NMR}\left(500 \mathrm{MHz}, \mathrm{CDl}_{3}\right) \delta 7.86(\mathrm{~d}, J=7.4 \mathrm{~Hz}, 2 \mathrm{H})$, $7.75(\mathrm{~s}, 1 \mathrm{H}), 7.47-7.56(\mathrm{~m}, 4 \mathrm{H}), 7.41(\mathrm{~d}, J=7.4 \mathrm{~Hz}, 1 \mathrm{H}), 6.97(\mathrm{~d}, J=7.4 \mathrm{~Hz}, 1 \mathrm{H}), 2.37(\mathrm{~s}, 3 \mathrm{H}) .{ }^{13} \mathrm{C}\left\{{ }^{1} \mathrm{H}\right\}-$ NMR $\left(125 \mathrm{MHz} \mathrm{CDCl}_{3}\right) \delta 165.8,139.1,137.9,135.2,131.9,129.0,128.9,127.0,125.5,120.9,117.3,21.6$.

$N$-phenylbenzamide (4ga, Ref. 9)

White solid (84\%, $83 \mathrm{mg})$. m.p. $163{ }^{\circ} \mathrm{C}-164^{\circ} \mathrm{C} ;{ }^{1} \mathrm{H}-\mathrm{NMR}\left(500 \mathrm{MHz}, \mathrm{CDCl}_{3}\right) \delta 7.85-7.92(\mathrm{~m}, 3 \mathrm{H}), 7.64(\mathrm{~d}$, $J=8.0 \mathrm{~Hz}, 2 \mathrm{H}), 7.45-7.55(\mathrm{~m}, 3 \mathrm{H}), 7.36(\mathrm{t}, J=8.0 \mathrm{~Hz}, 2 \mathrm{H}), 7.15(\mathrm{t}, J=7.4 \mathrm{~Hz}, 1 \mathrm{H}) .{ }^{13} \mathrm{C}\left\{{ }^{1} \mathrm{H}\right\}-\mathrm{NMR}(125$ $\left.\mathrm{MHz}, \mathrm{CDCl}_{3}\right) \delta 165.9,138.0,134.9,131.9,129.2,128.9,127.1,124.7,120.3$. 
White solid (92\%, $105 \mathrm{mg})$. m.p. $157^{\circ} \mathrm{C}-158^{\circ} \mathrm{C} ;{ }^{1} \mathrm{H}-\mathrm{NMR}\left(500 \mathrm{MHz}, \mathrm{CDCl}_{3}\right) \delta 7.85(\mathrm{~d}, J=7.4 \mathrm{~Hz}$, 2H), 7.77 (brs, 1H), 7.45-7.54 (m, 5H), 6.89-6.91 (m, 2H), 3.81 (s, 3H). ${ }^{13} \mathrm{C}\left\{{ }^{1} \mathrm{H}\right\}-\mathrm{NMR}(125 \mathrm{MHz}$, $\left.\mathrm{CDCl}_{3}\right) \delta 165.7,156.7,135.0,131.8,131.1,128.8,127.1,122.2,114.3,55.6$.

$N$-(naphthalen-2-yl)benzamide (4ya, Ref. 16)

White Solid (77\%, $95 \mathrm{mg})$. m.p. $164^{\circ} \mathrm{C}-165^{\circ} \mathrm{C} ;{ }^{1} \mathrm{H}-\mathrm{NMR}\left(500 \mathrm{MHz}, \mathrm{CDCl}_{3}\right) \delta 8.22-8.41(\mathrm{~m}, 1 \mathrm{H}), 8.03$ (brs, 1H), 7.79-7.92 (m, 5H), 7.41-7.60 (m, 6H). ${ }^{13} \mathrm{C}\left\{{ }^{1} \mathrm{H}\right\}-\mathrm{NMR}\left(125 \mathrm{MHz}, \mathrm{CDCl}_{3}\right) \delta 166.1,135.5$, 135.1, 134.0, 132.0, 130.89, 129.0, 127.8, 127.7, 127.5, 127.1, 126.7, 125.3, 120.2, 117.1.

$N$-(1-methyl-1H-indol-5-yl)benzamide (4wa, Ref. 17)

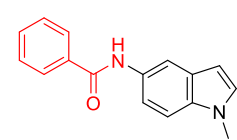

Reddish solid (89\%, $125 \mathrm{mg})$. m. p. $176^{\circ} \mathrm{C}-177^{\circ} \mathrm{C} ;{ }^{1} \mathrm{H}-\mathrm{NMR}\left(500 \mathrm{MHz}, \mathrm{CDCl}_{3}\right) \delta$ 7.87-7.94 (m, 4H), 7.28-7.51 (m, 5H), 7.00-7.15 (m, 1H), 6.39-6.51 (m, 1H), $3.78(\mathrm{~s}, 3 \mathrm{H}) .{ }^{13} \mathrm{C}\left\{{ }^{1} \mathrm{H}\right\}-\mathrm{NMR}(125 \mathrm{MHz}$, $\left.\mathrm{CDCl}_{3}\right) \delta 165.8,134.6,131.6,130.2,129.9,129.0,128.8,128.0,127.1,116.2,113.3,109.5,101.2,33.1$.

2-methyl- $N$-(p-tolyl)benzamide (4ad, Ref. 14)

White solid (98\%, $104 \mathrm{mg})$. m.p. $142^{\circ} \mathrm{C}-143^{\circ} \mathrm{C} ;{ }^{1} \mathrm{H}-\mathrm{NMR}\left(500 \mathrm{MHz}, \mathrm{CDCl}_{3}\right) \delta$ 7.46-7.50 (m, 3H), 7.41 (brs, 1H), $7.35(\mathrm{t}, J=7.2 \mathrm{~Hz}, 1 \mathrm{H}), 7.23-7.26(\mathrm{~m}, 2 \mathrm{H}), 7.16(\mathrm{~d}, J=7.2 \mathrm{~Hz}, 2 \mathrm{H}), 2.50(\mathrm{~s}, 3 \mathrm{H}), 2.34(\mathrm{~s}, 3 \mathrm{H})$. ${ }^{13} \mathrm{C}\left\{{ }^{1} \mathrm{H}\right\}-\mathrm{NMR}\left(125 \mathrm{MHz}, \mathrm{CDCl}_{3}\right) \delta 168.0,136.7,136.5,135.5,134.3,131.3,130.3,129.7,126.7,126.0,120.0,21.0,19.9$. $N$-(pyridin-3-yl)benzamide (4va, Ref. 18)

White solid $(88 \%, 88 \mathrm{mg})$. m.p. $116^{\circ} \mathrm{C}-118^{\circ} \mathrm{C} ;{ }^{1} \mathrm{H}-\mathrm{NMR}\left(500 \mathrm{MHz}, \mathrm{CDCl}_{3}\right) \delta 8.67(\mathrm{~d}, J=2.3 \mathrm{~Hz}, 1 \mathrm{H})$, 8.28-8.35 (m, 2H), $7.88(\mathrm{~d}, J=7.4 \mathrm{~Hz}, 2 \mathrm{H}), 7.44-7.57(\mathrm{~m}, 3 \mathrm{H}), 7.25-7.32(\mathrm{~m}, 1 \mathrm{H}) .{ }^{13} \mathrm{C}\left\{{ }^{1} \mathrm{H}\right\}-\mathrm{NMR}(125$ $\left.\mathrm{MHz}, \mathrm{CDCl}_{3}\right) \delta 166.5,145.6,141.7,134.3,132.3,129.0,128.7,127.8,127.3,123.7$.

4-methoxy- $N$-(p-tolyl)benzamide (4ae, Ref. 14)

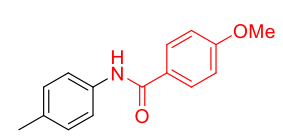

White solid (99\%, $119 \mathrm{mg})$. m.p. $149^{\circ} \mathrm{C}-150{ }^{\circ} \mathrm{C} ;{ }^{1} \mathrm{H}-\mathrm{NMR}\left(500 \mathrm{MHz}, \mathrm{CDCl}_{3}\right) \delta 7.83-7.81$ (m, 3H), $7.50(\mathrm{~d}, J=8.6 \mathrm{~Hz}, 2 \mathrm{H}), 7.15(\mathrm{~d}, J=8.6 \mathrm{~Hz}, 2 \mathrm{H}), 6.94(\mathrm{~d}, J=8.6 \mathrm{~Hz}, 2 \mathrm{H}), 3.85(\mathrm{~s}, 3 \mathrm{H}), 2.33(\mathrm{~s}, 3 \mathrm{H})$. ${ }^{13} \mathrm{C}\left\{{ }^{1} \mathrm{H}\right\}-\mathrm{NMR}(125 \mathrm{MHz}, \mathrm{CDCl} 3) \delta 165.6,162.5,135.6,134.1,129.6,129.0,127.3,120.4,114.0$,

$55.5,21.0$.

$N$-(3-(trifluoromethyl)phenyl)benzamide (4ma, Ref. 19)

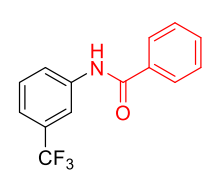

White solid (86\%, $114 \mathrm{mg})$. m.p. $111^{\circ} \mathrm{C}-112^{\circ} \mathrm{C}$; ${ }^{1} \mathrm{H}-\mathrm{NMR}\left(500 \mathrm{MHz}, \mathrm{CDCl}_{3}\right) \delta 8.03$ (brs, 1H), 7.89-7.94 $(\mathrm{m}, 1 \mathrm{H}), 7.87-7.85(\mathrm{~m}, 3 \mathrm{H}), 7.56(\mathrm{dd}, J=6.3,2.6 \mathrm{~Hz}, 1 \mathrm{H}), 7.39-7.49(\mathrm{~m}, 4 \mathrm{H}) .{ }^{13} \mathrm{C}\left\{{ }^{1} \mathrm{H}\right\}-\mathrm{NMR}(125 \mathrm{MHz}$, $\left.\mathrm{CDCl}_{3}\right) \delta 166.0,138.5,134.4,132.3,131.8\left(\mathrm{q}, J^{F}=32.6 \mathrm{~Hz}\right), 129.7,129.0,127.1,123.3\left(\mathrm{q}, J^{F}=272.9\right.$ $\mathrm{Hz}), 121.2\left(\mathrm{q}, J^{F}=3.2 \mathrm{~Hz}\right), 117.0\left(\mathrm{q}, J^{F}=3.6 \mathrm{~Hz}\right) .{ }^{19} \mathrm{~F}-\mathrm{NMR}\left(470 \mathrm{MHz}, \mathrm{CDCl}_{3}\right): \delta-62.60$.

$N$-(4-(trifluoromethyl)phenyl)benzamide (4la, Ref. 9)

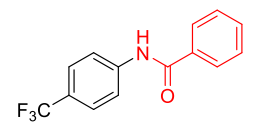

White solid (56\%, $74 \mathrm{mg})$. m.p. $205^{\circ} \mathrm{C}-206^{\circ} \mathrm{C} ;{ }^{1} \mathrm{H}-\mathrm{NMR}\left(500 \mathrm{MHz}, \mathrm{CDCl}_{3}\right) \delta 7.97$ (brs, 1H), $7.88(\mathrm{~d}$, $J=7.4 \mathrm{~Hz}, 2 \mathrm{H}), 7.79(\mathrm{~d}, J=8.0 \mathrm{~Hz}, 2 \mathrm{H}), 7.63(\mathrm{~d}, J=8.0 \mathrm{~Hz}, 2 \mathrm{H}), 7.59(\mathrm{td}, J=7.4,1.3 \mathrm{~Hz}, 1 \mathrm{H}), 7.51$ $(\mathrm{t}, J=7.4 \mathrm{~Hz}, 2 \mathrm{H}) .{ }^{13} \mathrm{C}\left\{{ }^{1} \mathrm{H}\right\}-\mathrm{NMR}\left(125 \mathrm{MHz}, \mathrm{CDCl}_{3}\right) \delta 166.0,141.0,134.5,132.4,129.0,127.2,126.8$ $\left(\mathrm{q}, J^{F}=3.8 \mathrm{~Hz}\right), 126.6\left(\mathrm{~d}, J^{F}=224.5 \mathrm{~Hz}\right), 126.5\left(\mathrm{~d}, J^{F}=12.3 \mathrm{~Hz}\right), 119.7 .{ }^{19} \mathrm{~F}-\mathrm{NMR}\left(470 \mathrm{MHz}, \mathrm{CDCl}_{3}\right): \delta-62.01$.

$N$-(3-chlorophenyl)benzamide (4ja, Ref. 9)

White solid (81\%, $95 \mathrm{mg})$. m.p. $120^{\circ} \mathrm{C}-121^{\circ} \mathrm{C} ;{ }^{1} \mathrm{H}-\mathrm{NMR}\left(500 \mathrm{MHz}, \mathrm{CDCl}_{3}\right) \delta$ 7.86-7.84 (m, 3H), $7.77(\mathrm{t}$, $J=2.0 \mathrm{~Hz}, 1 \mathrm{H}), 7.56(\mathrm{t}, J=8.0 \mathrm{~Hz}, 1 \mathrm{H}), 7.49(\mathrm{t}, J=6.6 \mathrm{~Hz}, 3 \mathrm{H}), 7.28(\mathrm{t}, J=8.0 \mathrm{~Hz}, 1 \mathrm{H}), 7.13(\mathrm{~d}, J=8.0$ $\mathrm{Hz}, 1 \mathrm{H}) .{ }^{13} \mathrm{C}\left\{{ }^{1} \mathrm{H}\right\}-\mathrm{NMR}\left(125 \mathrm{MHz}, \mathrm{CDCl}_{3}\right) \delta 165.8,139.1,134.9,134.6,132.2,130.2,129.0,127.1,124.7$,

120.4, 118.2. 
White Solid (99\%, $112 \mathrm{mg})$. m.p. $160^{\circ} \mathrm{C}-161^{\circ} \mathrm{C} ;{ }^{1} \mathrm{H}-\mathrm{NMR}\left(500 \mathrm{MHz}, \mathrm{CDCl}_{3}\right) \delta 7.81$ (brs, $\left.1 \mathrm{H}\right), 7.75(\mathrm{~d}$, $J=8.0 \mathrm{~Hz}, 2 \mathrm{H}), 7.51(\mathrm{~d}, J=8.0 \mathrm{~Hz}, 2 \mathrm{H}), 7.26(\mathrm{~d}, J=8.0 \mathrm{~Hz}, 2 \mathrm{H}), 7.15(\mathrm{~d}, J=8.0 \mathrm{~Hz}, 2 \mathrm{H}), 2.41(\mathrm{~s}$, $3 \mathrm{H}), 2.33$ (s, 3H). ${ }^{13} \mathrm{C}\left\{{ }^{1} \mathrm{H}\right\}-\mathrm{NMR}\left(125 \mathrm{MHz}, \mathrm{CDCl}_{3}\right) \delta 165.7,142.3,135.6,134.2,132.3,129.7,129.5,127.1,120.4,21.6$, 21.00 .

\section{$N$-(naphthalen-1-yl)benzamide (4xa, Ref. 18)}

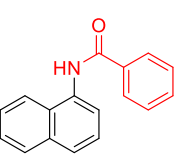

White solid $(42 \%, 52 \mathrm{mg})$. m.p. $159^{\circ} \mathrm{C}-160^{\circ} \mathrm{C} ;{ }^{1} \mathrm{H}-\mathrm{NMR}\left(500 \mathrm{MHz}, \mathrm{CDCl}_{3}\right) \delta 8.19$ (brs, $\left.1 \mathrm{H}\right), 8.06(\mathrm{~d}, J=$ $5.6 \mathrm{~Hz}, 1 \mathrm{H}), 8.00(\mathrm{~d}, J=6.9 \mathrm{~Hz}, 2 \mathrm{H}), 7.91(\mathrm{t}, J=6.9 \mathrm{~Hz}, 2 \mathrm{H}), 7.76(\mathrm{~d}, J=8.0 \mathrm{~Hz}, 1 \mathrm{H}), 7.52-7.60(\mathrm{~m}, 6 \mathrm{H})$.

${ }^{13} \mathrm{C}\left\{{ }^{1} \mathrm{H}\right\}$-NMR $(125 \mathrm{MHz}, \mathrm{CDCl} 3) \delta 166.4,135.7,135.0,134.3,132.5,132.1,129.0,128.1,127.3,126.5$, $126.3,126.2,125.9,121.4,120.8$.

$N$-(4-chlorophenyl)benzamide (4ia, Ref. 12)

white solid (66\%, $77 \mathrm{mg})$. m.p. $187^{\circ} \mathrm{C}-188^{\circ} \mathrm{C} ;{ }^{1} \mathrm{H}-\mathrm{NMR}\left(500 \mathrm{MHz}, \mathrm{CDCl}_{3}\right) \delta 7.82-7.86(\mathrm{~m}, 3 \mathrm{H}), 7.49$ $7.61(\mathrm{~m}, 5 \mathrm{H}), 7.25-7.34(\mathrm{~m}, 2 \mathrm{H}) .{ }^{13} \mathrm{C}\left\{{ }^{1} \mathrm{H}\right\}-\mathrm{NMR}\left(125 \mathrm{MHz}, \mathrm{CDCl}_{3}\right) \delta 165.8,136.6,134.7,132.2,129.6$, 129.2, 129.0, 127.1, 121.5.

$N$-(4-bromophenyl)benzamide (4ha, Ref. 11)

White solid (72\%, $99 \mathrm{mg})$. m.p. $205^{\circ} \mathrm{C}-206^{\circ} \mathrm{C} ;{ }^{1} \mathrm{H}-\mathrm{NMR}\left(500 \mathrm{MHz}, \mathrm{CDCl}_{3}\right) \delta 7.85(\mathrm{~d}, \mathrm{~J}=8.6 \mathrm{~Hz}, 2 \mathrm{H})$, 7.78 (brs, $1 \mathrm{H}), 7.47-7.57(\mathrm{~m}, 7 \mathrm{H}) .{ }^{13} \mathrm{C}\left\{{ }^{1} \mathrm{H}\right\}-\mathrm{NMR}\left(125 \mathrm{MHz}, \mathrm{CDCl}_{3}\right.$ with 2 drops methanol-d $\left.\mathrm{d}_{3}\right) \delta 171.1$, $141.55,138.6,135.9,135.7,132.5,131.3,126.3,121.0$.

$\mathrm{N}$-(4-methoxyphenyl)acetamide (4eh, Ref. 20)

White solid (89\%, $74 \mathrm{mg})$. m.p. $127^{\circ} \mathrm{C}-129^{\circ} \mathrm{C} ;{ }^{1} \mathrm{H}-\mathrm{NMR}\left(500 \mathrm{MHz}, \mathrm{CDCl}_{3}\right) \delta 7.38(\mathrm{dd}, J=8.6,2.0 \mathrm{~Hz}$, 2H), 7.06 (brs, 1H), 6.85 (d, $J=8.6 \mathrm{~Hz}, 2 \mathrm{H}), 3.78(\mathrm{~s}, 3 \mathrm{H}), 2.15(\mathrm{~s}, 3 \mathrm{H}) .{ }^{13} \mathrm{C}\left\{{ }^{1} \mathrm{H}\right\}-\mathrm{NMR}\left(125 \mathrm{MHz}, \mathrm{CDCl}_{3}\right)$ $\delta 168.4,156.5,131.0,122.0,114.2,55.6,24.4$.

$N$-(4-butylphenyl)benzamide (4da, Ref. 21)

White solid (71\%, $90 \mathrm{mg})$. m.p. $111^{\circ} \mathrm{C}-112{ }^{\circ} \mathrm{C} ;{ }^{1} \mathrm{H}-\mathrm{NMR}\left(500 \mathrm{MHz}, \mathrm{CDCl}_{3}\right) \delta 7.85(\mathrm{~d}, J=7.4 \mathrm{~Hz}$, 2H), 7.77 (brs, 1H), 7.46-7.54 (m, 5H), $7.18(\mathrm{~d}, J=7.4 \mathrm{~Hz}, 2 \mathrm{H}), 2.59$ (t, $J=7.4 \mathrm{~Hz}, 2 \mathrm{H}), 1.60(\mathrm{t}, J$ $=7.4 \mathrm{~Hz}, 2 \mathrm{H}$ ), $1.33-1.39$ (quint, $J=7.4 \mathrm{~Hz}, 2 \mathrm{H}), 0.93(\mathrm{t}, J=7.4 \mathrm{~Hz}, 3 \mathrm{H}) .{ }^{13} \mathrm{C}\left\{{ }^{1} \mathrm{H}\right\}-\mathrm{NMR}\left(125 \mathrm{MHz}, \mathrm{CDCl}_{3}\right) \delta 165.8,139.5$, $135.7,135.6,131.8,129.1,128.8,127.1,120.4,35.2,33.7,22.4,14.0$.

ethyl 4-benzamidobenzoate (4oa, Ref. 11)

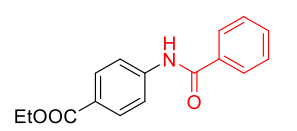

White solid $(61 \%, 83 \mathrm{mg})$. m.p. $149^{\circ} \mathrm{C}-150^{\circ} \mathrm{C} ;{ }^{1} \mathrm{H}-\mathrm{NMR}\left(500 \mathrm{MHz}, \mathrm{CDCl}_{3}\right) \delta 8.00-8.07$ (m, 3H), $7.86(\mathrm{t}, J=5.7 \mathrm{~Hz}, 2 \mathrm{H}), 7.73-7.75(\mathrm{~m}, 2 \mathrm{H}), 7.48-7.59(\mathrm{~m}, 3 \mathrm{H}), 4.36(\mathrm{q}, J=6.4 \mathrm{~Hz}, 2 \mathrm{H}), 1.39(\mathrm{t}, J=$ $6.4 \mathrm{~Hz}, 3 \mathrm{H}) .{ }^{13} \mathrm{C}\left\{{ }^{1} \mathrm{H}\right\}-\mathrm{NMR}\left(125 \mathrm{MHz}, \mathrm{CDCl}_{3}\right) \delta 166.1,165.7,142.1,134.5,132.3,131.0,129.0$,

127.2, 126.2, 119.2, 61.0, 14.4 .

$N$-(p-tolyl)hex-5-enamide (4ak, Ref. 22)

White solid $(87 \%, 89 \mathrm{mg})$. m.p. $55^{\circ} \mathrm{C}-57^{\circ} \mathrm{C} ;{ }^{1} \mathrm{H}-\mathrm{NMR}\left(500 \mathrm{MHz}, \mathrm{CDCl}_{3}\right) \delta 7.37$ (d, $\left.J=8.5 \mathrm{~Hz}, 2 \mathrm{H}\right)$, $7.24(\mathrm{brs}, 1 \mathrm{H}), 7.10(\mathrm{~d}, J=8.5 \mathrm{~Hz}, 2 \mathrm{H}), 5.75-5.84(\mathrm{~m}, 1 \mathrm{H}), 4.99-5.06(\mathrm{~m}, 2 \mathrm{H}), 2.30-2.35(\mathrm{~m}, 5 \mathrm{H})$, $2.14(\mathrm{q}, J=7.2 \mathrm{~Hz}, 2 \mathrm{H}), 1.79-1.85$ (quint, $J=7.2,2 \mathrm{H}) .{ }^{13} \mathrm{C}\left\{{ }^{1} \mathrm{H}\right\}-\mathrm{NMR}\left(125 \mathrm{MHz}, \mathrm{CDCl}_{3}\right) \delta 171.2,138.0,135.4,133.9$, $129.6,120.0,115.6,36.9,33.2,24.7,21.0$.

$N$-(p-tolyl)hexanamide (4aj, Ref. 23)

White solid (84\%, $87 \mathrm{mg})$. m.p. $73^{\circ} \mathrm{C}-74{ }^{\circ} \mathrm{C} ;{ }^{1} \mathrm{H}-\mathrm{NMR}\left(500 \mathrm{MHz}, \mathrm{CDCl}_{3}\right) \delta 7.39$ (d, J=7.2 Hz, 2H), $7.22(\mathrm{~s}, 1 \mathrm{H}), 7.08(\mathrm{~d}, J=7.2 \mathrm{~Hz}, 2 \mathrm{H}), 2.21-2.43(\mathrm{~m}, 5 \mathrm{H}), 1.59-1.81(\mathrm{~m}, 2 \mathrm{H}), 1.20-1.45(\mathrm{~m}, 4 \mathrm{H}), 0.89$ 
$(\mathrm{t}, J=1.7 \mathrm{~Hz}, 3 \mathrm{H}) .{ }^{13} \mathrm{C}\left\{{ }^{1} \mathrm{H}\right\}-\mathrm{NMR}\left(125 \mathrm{MHz}, \mathrm{CDCl}_{3}\right) \delta 171.5,135.2,133.7,129.5,120.0,37.8,31.5,25.5,22.5,21.0$, 14.0 .

$N$-(4-ethoxyphenyl)benzamide (4fa, Ref. 15)

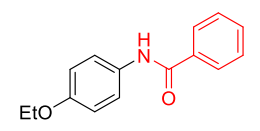

White solid (94\%, $113 \mathrm{mg})$. m.p. $171^{\circ} \mathrm{C}-172{ }^{\circ} \mathrm{C} ;{ }^{1} \mathrm{H}-\mathrm{NMR}\left(500 \mathrm{MHz}, \mathrm{CDCl}_{3}\right) \delta 7.85(\mathrm{~d}, J=4.6 \mathrm{~Hz}$, 2H), 7.70 (brs, 1H), 7.41-7.58 (m, 5H), 6.89-6.91 (m, 2H), 4.03 (q, J=6.6 Hz, 2H), $1.41(\mathrm{t}, J=6.6 \mathrm{~Hz}$,

$3 \mathrm{H}) .{ }^{13} \mathrm{C}\left\{{ }^{1} \mathrm{H}\right\}-\mathrm{NMR}\left(125 \mathrm{MHz}, \mathrm{CDCl}_{3}\right) \delta 165.7,155.9,135.0,131.7,131.0,128.8,127.1,122.2,114.9$,

$63.8,14.9$.

$N$-(o-tolyl)benzamide (4ca, Ref. 14)

White solid (93\%, $98 \mathrm{mg})$. m.p. $145^{\circ} \mathrm{C}-146^{\circ} \mathrm{C} ;{ }^{1} \mathrm{H}-\mathrm{NMR}\left(500 \mathrm{MHz}, \mathrm{CDCl}_{3}\right) \delta$ 7.77-7.96 (m, 3H), 7.68 (brs, 1H), 7.43-7.62 (m, 3H), 7.22-7.26 (m, 2H), $7.13(\mathrm{~d}, J=6.9 \mathrm{~Hz}, 1 \mathrm{H}), 2.34(\mathrm{~s}, 3 \mathrm{H}) .{ }^{13} \mathrm{C}\left\{{ }^{1} \mathrm{H}\right\}-\mathrm{NMR}(125$ $\left.\mathrm{MHz}, \mathrm{CDCl}_{3}\right) \delta 165.6,135.9,135.0,131.9,130.7,129.3,128.9,127.1,127.0,125.5,123.2,17.9$.

$N$-(4-(trifluoromethyl)phenyl)acetamide (4lh, Ref. 24)

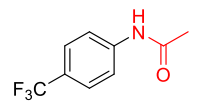

White solid (75\%, $76 \mathrm{mg})$. m.p. $152^{\circ} \mathrm{C}-153^{\circ} \mathrm{C} ;{ }^{1} \mathrm{H}-\mathrm{NMR}\left(500 \mathrm{MHz}, \mathrm{CDCl}_{3}\right) \delta 7.63(\mathrm{~d}, J=8.0 \mathrm{~Hz}, 2 \mathrm{H})$, $7.57(\mathrm{~d}, J=8.0 \mathrm{~Hz}, 2 \mathrm{H}), 2.21(\mathrm{~s}, 3 \mathrm{H}) .{ }^{13} \mathrm{C}\left\{{ }^{1} \mathrm{H}\right\}-\mathrm{NMR}\left(125 \mathrm{MHz}, \mathrm{CDCl}_{3}\right) \delta 168.6,141.0,126.4\left(\mathrm{q}, J_{\mathrm{C}-\mathrm{F}}=\right.$ $4.0 \mathrm{~Hz}), 119.4,24.8 .{ }^{19} \mathrm{~F}-\mathrm{NMR}\left(470 \mathrm{MHz}, \mathrm{CDCl}_{3}\right): \delta-62.04$.

$N$-(4-(hydroxymethyl)phenyl)benzamide (4ra, Ref. 25)

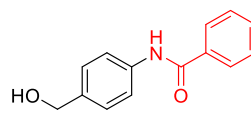

White solid (55\%, $63 \mathrm{mg})$. m.p. $104^{\circ} \mathrm{C}-106^{\circ} \mathrm{C} ;{ }^{1} \mathrm{H}-\mathrm{NMR}\left(500 \mathrm{MHz}, \mathrm{METHANOL}-d_{3}\right) \delta 7.88(\mathrm{dd}, J$ $=8.2 \mathrm{~Hz}, 2.4 \mathrm{~Hz}, 2 \mathrm{H}), 7.66(\mathrm{~d}, J=8.6 \mathrm{~Hz}, 2 \mathrm{H}), 7.42-7.56(\mathrm{~m}, 3 \mathrm{H}), 7.34(\mathrm{~d}, J=8.2 \mathrm{~Hz}, 2 \mathrm{H}), 4.57(\mathrm{~s}$, $2 \mathrm{H}) .{ }^{13} \mathrm{C}\left\{{ }^{1} \mathrm{H}\right\}$-NMR $\left(125 \mathrm{MHz}, \mathrm{METHANOL}-d_{3}\right) \delta 169.1,139.2,136.5,135.2,133.1,129.7,128.8,128.8,122.4,65.1$.

$N$-(4-(dimethylamino)phenyl)benzamide (4sa, Ref. 26)

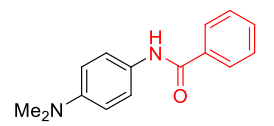

White solid (43\%, $52 \mathrm{mg})$. m.p. $226^{\circ} \mathrm{C}-227^{\circ} \mathrm{C} ;{ }^{1} \mathrm{H}-\mathrm{NMR}\left(500 \mathrm{MHz}, \mathrm{METHANOL}-d_{3}\right) \delta 7.89(\mathrm{~d}, J=$ $6.9 \mathrm{~Hz}, 2 \mathrm{H}), 7.46-7.59(\mathrm{~m}, 5 \mathrm{H}), 6.78(\mathrm{~d}, J=9.2 \mathrm{~Hz}, 2 \mathrm{H}), 3.29(\mathrm{~s}, 6 \mathrm{H}) .{ }^{13} \mathrm{C}\left\{{ }^{1} \mathrm{H}\right\}-\mathrm{NMR}(125 \mathrm{MHz}$, DMSO- $\left.d_{6}\right) \delta 165.8,148.2,131.9,129.0,128.6,127.9,122.7,113.1,40.9$.

\subsection{General Procedure for Suzuki-Miyaura-Type Cross-Coupling Reaction}

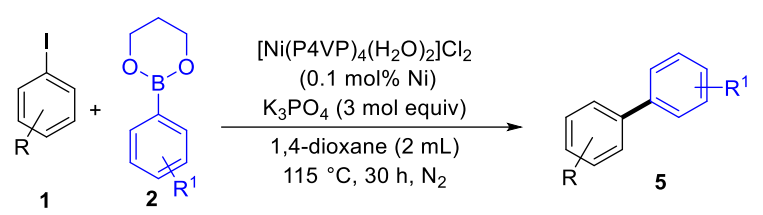

A mixture of $\left[\mathrm{Ni}(\mathrm{P} 4 \mathrm{VP})_{4}\left(\mathrm{H}_{2} \mathrm{O}\right)_{2}\right] \mathrm{Cl}_{2}(1000 \mathrm{~mol} \mathrm{ppm}, 0.42 \mathrm{mg})$, aryl iodide, 1 (1 mol equiv, $\left.0.5 \mathrm{mmol}\right)$, arylboronic ester, 2 (5 mol equiv, $2.5 \mathrm{mmol}$ ), and $\mathrm{K}_{3} \mathrm{PO}_{4}(3 \mathrm{~mol}$ equiv, $1.5 \mathrm{mmol}, 318 \mathrm{mg}$ ) were added to a reaction tube. The reaction tube was degassed under vacuum and refile with $\mathrm{N}_{2}$ under standard Schlack techniques (3 times). 1,4-dioxane (2 mL) was added and seal the reaction tube with teflon, followed by stirring at $115^{\circ} \mathrm{C}$ (chemiStation) under nitrogen for $30 \mathrm{~h}$. After that the reaction mixture was diluted by EtOAc and extracted with $\mathrm{H}_{2} \mathrm{O}$. The organic layer was collected and dried over $\mathrm{Na}_{2} \mathrm{SO}_{4}$ and filtered. The solvent was evaporated under vacuum and crude mass was purified by column chromatography to give products 5 .

\subsection{Characterization of Products}

4-methyl-1,1'-biphenyl (5aa, Ref. 27) 
White solid (91\%, $76 \mathrm{mg})$. m.p. $47^{\circ} \mathrm{C}-48^{\circ} \mathrm{C} ;{ }^{1} \mathrm{H}-\mathrm{NMR}\left(500 \mathrm{MHz}, \mathrm{CDCl}_{3}\right) \delta 7.62(\mathrm{~d}, J=7.4 \mathrm{~Hz}, 2 \mathrm{H}), 7.54(\mathrm{~d}$, $J=7.4 \mathrm{~Hz}, 2 \mathrm{H}), 7.46(\mathrm{t}, J=7.4 \mathrm{~Hz}, 2 \mathrm{H}), 7.36(\mathrm{t}, J=6.6 \mathrm{~Hz}, 1 \mathrm{H}), 7.29(\mathrm{~d}, J=8.0 \mathrm{~Hz}, 2 \mathrm{H}), 2.44(\mathrm{~s}, 3 \mathrm{H})$. ${ }^{13} \mathrm{C}\left\{{ }^{1} \mathrm{H}\right\}-\mathrm{NMR}\left(125 \mathrm{MHz}, \mathrm{CDCl}_{3}\right) \delta 141.3,138.5,137.1,129.6,128.8,127.1,21.2$.

4-methoxy-1,1'-biphenyl (5ea, Ref. 27)

White solid (92\%, $85 \mathrm{mg})$. m.p $89^{\circ} \mathrm{C}-90^{\circ} \mathrm{C} ;{ }^{1} \mathrm{H}-\mathrm{NMR}\left(500 \mathrm{MHz}, \mathrm{CDCl}_{3}\right) \delta 7.53-7.57(\mathrm{~m}, 4 \mathrm{H}), 7.43(\mathrm{t}, J=$ $7.2 \mathrm{~Hz}, 2 \mathrm{H}), 7.31(\mathrm{t}, J=7.2 \mathrm{~Hz}, 1 \mathrm{H}), 6.99$ (d, $J=8.6 \mathrm{~Hz}, 2 \mathrm{H}), 3.86(\mathrm{~s}, 3 \mathrm{H}) .{ }^{13} \mathrm{C}\left\{{ }^{1} \mathrm{H}\right\}-\mathrm{NMR}(125 \mathrm{MHz}$, $\left.\mathrm{CDCl}_{3}\right) \delta 159.0,141.1,133.4,128.8,128.3,126.8,126.8,114.3,55.6$.

\section{1-([1,1'-biphenyl]-4-yl)ethan-1-one (5na, Ref. 27)}

White solid $(63 \%, 62 \mathrm{mg})$. m.p. $116^{\circ} \mathrm{C}-117^{\circ} \mathrm{C} ;{ }^{1} \mathrm{H}-\mathrm{NMR}\left(500 \mathrm{MHz}, \mathrm{CDCl}_{3}\right) \delta 8.03(\mathrm{~d}, J=8.6 \mathrm{~Hz}, 2 \mathrm{H})$, $7.68(\mathrm{~d}, J=8.6 \mathrm{~Hz}, 2 \mathrm{H}), 7.63(\mathrm{~d}, J=8.0 \mathrm{~Hz}, 2 \mathrm{H}), 7.47(\mathrm{t}, J=7.8 \mathrm{~Hz}, 2 \mathrm{H}), 7.40(\mathrm{t}, J=7.8 \mathrm{~Hz}, 1 \mathrm{H}), 2.64(\mathrm{~s}$, $3 \mathrm{H}) .{ }^{13} \mathrm{C}\left\{{ }^{1} \mathrm{H}\right\}-\mathrm{NMR}\left(125 \mathrm{MHz}, \mathrm{CDCl}_{3}\right) \delta 197.9,145.9,134.0,135.9,129.1,129.0,128.3,127.4,127.3$,

26.8 .

\section{4-ethoxy-1,1'-biphenyl (5fa, Ref. 27)}

White solid (94\%, $93 \mathrm{mg})$. m.p. $72{ }^{\circ} \mathrm{C}-73^{\circ} \mathrm{C} ;{ }^{1} \mathrm{H}-\mathrm{NMR}\left(500 \mathrm{MHz}, \mathrm{CDCl}_{3}\right) \delta 7.51-7.58(\mathrm{~m}, 4 \mathrm{H}), 7.42$ (q, $J$ $=7.4 \mathrm{~Hz}, 2 \mathrm{H}), 7.28(\mathrm{t}, J=7.4 \mathrm{~Hz}, 1 \mathrm{H}), 6.98(\mathrm{t}, J=7.4 \mathrm{~Hz}, 2 \mathrm{H}), 4.06-4.12(\mathrm{~m}, 2 \mathrm{H}), 1.45(\mathrm{q}, J=7.4 \mathrm{~Hz}$, $3 \mathrm{H}) .{ }^{13} \mathrm{C}\left\{{ }^{1} \mathrm{H}\right\}-\mathrm{NMR}\left(125 \mathrm{MHz}, \mathrm{CDCl}_{3}\right) \delta 158.4,141.0,133.8,128.8,128.2,126.8,126.7,114.8,63.6,15.0$.

4-chloro-1,1'-biphenyl (5ia, Ref. 27)

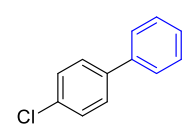

White solid (86\%, $81 \mathrm{mg})$. m.p. $72{ }^{\circ} \mathrm{C}-73{ }^{\circ} \mathrm{C} ;{ }^{1} \mathrm{H}-\mathrm{NMR}\left(500 \mathrm{MHz}, \mathrm{CDCl}_{3}\right) \delta 7.59(\mathrm{~d}, J=6.9 \mathrm{~Hz}, 3 \mathrm{H}), 7.44$ $(\mathrm{t}, J=7.6 \mathrm{~Hz}, 4 \mathrm{H}), 7.34(\mathrm{t}, J=7.6 \mathrm{~Hz}, 2 \mathrm{H}) .{ }^{13} \mathrm{C}\left\{{ }^{1} \mathrm{H}\right\}-\mathrm{NMR}\left(125 \mathrm{MHz}, \mathrm{CDCl}_{3}\right) \delta 140.1,139.8,133.5,129.0$, $128.5,127.7,127.1$.

\section{3-bromo-1,1'-biphenyl (5za, Ref. 27)}

White solid (77\%, $90 \mathrm{mg})$. m.p. $89^{\circ} \mathrm{C}-90^{\circ} \mathrm{C} ;{ }^{1} \mathrm{H}-\mathrm{NMR}\left(500 \mathrm{MHz}, \mathrm{CDCl}_{3}\right) \delta 7.77(\mathrm{~d}, J=1.7 \mathrm{~Hz}, 1 \mathrm{H}), 7.38-7.59$ $(\mathrm{m}, 7 \mathrm{H}), 7.32(\mathrm{t}, J=8.0 \mathrm{~Hz}, 1 \mathrm{H}) .{ }^{13} \mathrm{C}\left\{{ }^{1} \mathrm{H}\right\}-\mathrm{NMR}\left(125 \mathrm{MHz}, \mathrm{CDCl}_{3}\right) \delta 143.5,139.8,130.4,130.3,130.3,129.0$, 128.0, 127.3, 125.9, 123.0 .

\section{3,5-dichloro-1,1'-biphenyl (5ka, Ref. 28)}

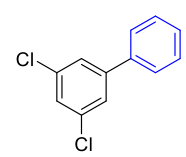

Colorless liquid (68\%, $76 \mathrm{mg}) .{ }^{1} \mathrm{H}-\mathrm{NMR}\left(500 \mathrm{MHz}, \mathrm{CDCl}_{3}\right) \delta 7.80(\mathrm{~s}, 1 \mathrm{H}), 7.72(\mathrm{~d}, J=8.0 \mathrm{~Hz}, 2 \mathrm{H}), 7.49$ $(\mathrm{t}, J=7.8 \mathrm{~Hz}, 2 \mathrm{H}), 7.40(\mathrm{t}, J=7.8 \mathrm{~Hz}, 1 \mathrm{H}), 6.96(\mathrm{~d}, J=1.7 \mathrm{~Hz}, 1 \mathrm{H}), 6.81(\mathrm{~s}, 2 \mathrm{H}) .{ }^{13} \mathrm{C}\left\{{ }^{1} \mathrm{H}\right\}-\mathrm{NMR}(125 \mathrm{MHz}$, $\left.\mathrm{CDCl}_{3}\right) \delta 144.3,138.6,135.3,129.1,128.5,127.2,127.2,125.8$.

\section{2-phenylnaphthalene (5ya, Ref. 27)}

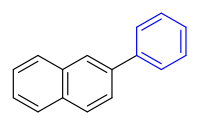

White solid (55\%, $56 \mathrm{mg})$. m.p. $102^{\circ} \mathrm{C}-103^{\circ} \mathrm{C} ;{ }^{1} \mathrm{H}-\mathrm{NMR}\left(500 \mathrm{MHz}, \mathrm{CDCl}_{3}\right) \delta 7.73-8.04(\mathrm{~m}, 6 \mathrm{H}), 7.26-$ $7.70(\mathrm{~m}, 6 \mathrm{H}) .{ }^{13} \mathrm{C}\left\{{ }^{1} \mathrm{H}\right\}-\mathrm{NMR}\left(125 \mathrm{MHz}, \mathrm{CDCl}_{3}\right) \delta 141.2,138.7,133.8,132.7,128.9,128.5,128.3,127.7$, $127.5,127.4,126.4,126.0,125.9,125.7$.

\section{3-phenylpyridine (5va, Ref. 29)}

Colorless liquid (89\%, $69 \mathrm{mg}) .{ }^{1} \mathrm{H}-\mathrm{NMR}\left(500 \mathrm{MHz}, \mathrm{CDCl}_{3}\right) \delta$ 9.00-8.71 (m, 1H), 8.71-8.39 (m, 1H), 7.95$7.75(\mathrm{~m}, 1 \mathrm{H}), 7.38-7.59(\mathrm{~m}, 5 \mathrm{H}), 7.25-7.27(\mathrm{~m}, 1 \mathrm{H}) .{ }^{13} \mathrm{C}\left\{{ }^{1} \mathrm{H}\right\}-\mathrm{NMR}\left(125 \mathrm{MHz}, \mathrm{CDCl}_{3}\right) \delta 148.5,148.4,138.1$, $134.2,129.2,128.9,128.8,128.2,127.3$.

\section{1,1'-biphenyl (5ga, Ref. 27)}

White solid (90\%, $69 \mathrm{mg})$. m.p. $68^{\circ} \mathrm{C}-69^{\circ} \mathrm{C} ;{ }^{1} \mathrm{H}-\mathrm{NMR}\left(500 \mathrm{MHz}, \mathrm{CDCl}_{3}\right) \delta 7.59(\mathrm{~d}, J=7.4 \mathrm{~Hz}, 2 \mathrm{H}), 7.44(\mathrm{t}$, $J=7.7 \mathrm{~Hz}, 2 \mathrm{H}), 7.34(\mathrm{t}, J=7.2 \mathrm{~Hz}, 1 \mathrm{H}) .{ }^{13} \mathrm{C}\left\{{ }^{1} \mathrm{H}\right\}-\mathrm{NMR}\left(125 \mathrm{MHz}, \mathrm{CDCl}_{3}\right) \delta 141.4,128.8,127.4,127.3$.

\section{2-methyl-1,1'-biphenyl (5ca, Ref. 27)}


Colorless liquid (84\%, $65 \mathrm{mg}) .{ }^{1} \mathrm{H}-\mathrm{NMR}\left(500 \mathrm{MHz}^{\mathrm{C}} \mathrm{CDCl}_{3}\right) \delta$ 7.47-7.50 (m, 2H), 7.39-7.42 (m, 3H), 7.32$7.34(\mathrm{~m}, 4 \mathrm{H}), 2.36(\mathrm{~s}, 3 \mathrm{H}) .{ }^{13} \mathrm{C}\left\{{ }^{1} \mathrm{H}\right\}-\mathrm{NMR}\left(125 \mathrm{MHz}, \mathrm{CDCl}_{3}\right) \delta 142.2,142.1,135.5,130.5,130.0,129.4,128.2$, 127.4, 126.9, 125.9, 20.6.

3-methyl-1,1'-biphenyl (5ba, Ref. 27)

Colorless liquid (87\%, $73 \mathrm{mg}) .{ }^{1} \mathrm{H}-\mathrm{NMR}\left(500 \mathrm{MHz} \mathrm{CDCl}_{3}\right) \delta 7.62(\mathrm{~d}, J=7.4 \mathrm{~Hz}, 2 \mathrm{H}), 7.42-7.48(\mathrm{~m}, 4 \mathrm{H})$, $7.36(\mathrm{t}, J=7.4 \mathrm{~Hz}, 2 \mathrm{H}), 7.20(\mathrm{~d}, J=7.4 \mathrm{~Hz}, 1 \mathrm{H}), 2.46(\mathrm{~s}, 3 \mathrm{H}) .{ }^{13} \mathrm{C}\left\{{ }^{1} \mathrm{H}\right\}-\mathrm{NMR}\left(125 \mathrm{MHz}, \mathrm{CDCl}_{3}\right) \delta 141.5$, $141.4,141.4,138.4,128.9,128.8,128.8,128.1,127.3,124.4,21.6$.

4-butyl-1,1'-biphenyl (5da, Ref. 30)

Colorless liquid (74\%, $78 \mathrm{mg}) .{ }^{1} \mathrm{H}-\mathrm{NMR}\left(500 \mathrm{MHz}, \mathrm{CDCl}_{3}\right) \delta 7.58(\mathrm{~d}, J=7.4 \mathrm{~Hz}, 2 \mathrm{H}), 7.51(\mathrm{~d}, J=8.0$ $\mathrm{Hz}, 2 \mathrm{H}), 7.43(\mathrm{t}, J=7.4 \mathrm{~Hz}, 2 \mathrm{H}), 7.32(\mathrm{t}, J=7.4 \mathrm{~Hz}, 1 \mathrm{H}), 7.26(\mathrm{~d}, J=8.0 \mathrm{~Hz}, 2 \mathrm{H}), 2.66(\mathrm{t}, J=7.6 \mathrm{~Hz}$, $2 \mathrm{H}), 1.61-1.67(\mathrm{~m}, 2 \mathrm{H}), 1.40(\mathrm{td}, J=14.7,7.6 \mathrm{~Hz}, 2 \mathrm{H}), 0.95(\mathrm{t}, J=7.6 \mathrm{~Hz}, 3 \mathrm{H}) .{ }^{13} \mathrm{C}\left\{{ }^{1} \mathrm{H}\right\}-\mathrm{NMR}\left(125 \mathrm{MHz}, \mathrm{CDCl}_{3}\right) \delta 142.2$, $141.3,138.7,128.9,128.8,127.1,127.0,35.4,33.7,22.5,14.1$.

\section{4-bromo-1,1'-biphenyl (5ha, Ref. 27)}

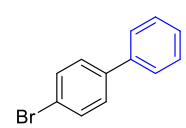

White solid (72\%, $84 \mathrm{mg})$. m.p. $91^{\circ} \mathrm{C}-92{ }^{\circ} \mathrm{C} ;{ }^{1} \mathrm{H}-\mathrm{NMR}\left(500 \mathrm{MHz}, \mathrm{CDCl}_{3}\right) \delta$ 7.54-7.57 (m, 4H), 7.42-7.47 (m, 4H), 7.35-7.38 (m, 1H). ${ }^{13} \mathrm{C}\left\{{ }^{1} \mathrm{H}\right\}-\mathrm{NMR}\left(125 \mathrm{MHz}, \mathrm{CDCl}_{3}\right) \delta 140.2,140.1,132.0,129.0,128.8,127.8$, 127.0, 121.6.

\section{4,4'-dimethyl-1,1'-biphenyl (5ag, Ref. 27)}

White solid (79\%, $72 \mathrm{mg})$. m.p. $121^{\circ} \mathrm{C}-122^{\circ} \mathrm{C} ;{ }^{1} \mathrm{H}-\mathrm{NMR}\left(500 \mathrm{MHz}, \mathrm{CDCl}_{3}\right) \delta 7.48(\mathrm{~d}, J=8.0 \mathrm{~Hz}, 2 \mathrm{H})$, $7.23(\mathrm{~d}, J=8.0 \mathrm{~Hz}, 2 \mathrm{H}), 2.39(\mathrm{~s}, 3 \mathrm{H}) .{ }^{13} \mathrm{C}\left\{{ }^{1} \mathrm{H}\right\}-\mathrm{NMR}\left(125 \mathrm{MHz}, \mathrm{CDCl}_{3}\right) \delta 138.4,136.8,129.5,126.9,21.2$.

4-methoxy-4'-methyl-1,1'-biphenyl (5ah, Ref. 27)

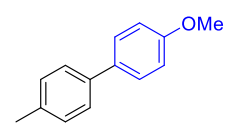

White solid (87\%, $86 \mathrm{mg})$. m.p. $104^{\circ} \mathrm{C}-105^{\circ} \mathrm{C} ;{ }^{1} \mathrm{H}-\mathrm{NMR}\left(500 \mathrm{MHz}, \mathrm{CDCl}_{3}\right) \delta$ 7.44-7.52 (m, 4H), 7.24 $(\mathrm{dd}, J=11.5,6.9 \mathrm{~Hz}, 2 \mathrm{H}), 6.97(\mathrm{dd}, J=6.9,2.3 \mathrm{~Hz}, 2 \mathrm{H}), 3.85(\mathrm{~s}, 3 \mathrm{H}), 2.38(\mathrm{~s}, 3 \mathrm{H}) .{ }^{13} \mathrm{C}\left\{{ }^{1} \mathrm{H}\right\}-\mathrm{NMR}(125$ $\left.\mathrm{MHz}, \mathrm{CDCl}_{3}\right) \delta 159.0,138.05,136.4,133.8,129.5,128.0,126.7,114.2,55.4,21.2$.

4-methyl-4' -(trifluoromethyl)-1,1'-biphenyl (5ai, Ref 27)

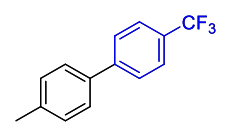

White solid (73\%, $86 \mathrm{mg})$. m.p. $125^{\circ} \mathrm{C}-126^{\circ} \mathrm{C} ;{ }^{1} \mathrm{H}-\mathrm{NMR}\left(500 \mathrm{MHz}, \mathrm{CDCl}_{3}\right) \delta$ 7.64-7.69 (m, 4H), 7.50 $(\mathrm{d}, J=8.0 \mathrm{~Hz}, 2 \mathrm{H}), 7.28(\mathrm{~d}, J=8.0 \mathrm{~Hz}, 2 \mathrm{H}), 2.41(\mathrm{~s}, 3 \mathrm{H}) .{ }^{13} \mathrm{C}\left\{{ }^{1} \mathrm{H}\right\}-\mathrm{NMR}\left(125 \mathrm{MHz}, \mathrm{CDCl}_{3}\right) \delta 144.7$, 138.23, 136.9, 129.8, $129.2\left(\mathrm{q}, J^{F}=32.5\right), 127.3,127.2,125.8\left(\mathrm{q}, J^{F}=4.0 \mathrm{~Hz}\right), 125.7,21.2 .{ }^{19} \mathrm{~F}-\mathrm{NMR}$ $\left(470 \mathrm{MHz}, \mathrm{CDCl}_{3}\right): \delta-62.23$.

\section{Reaction Procedure for Intermolcular Amidation}

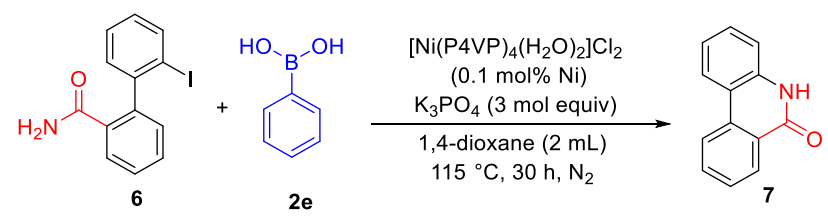

A mixture of $\left[\mathrm{Ni}(\mathrm{P} 4 \mathrm{VP})_{4}\left(\mathrm{H}_{2} \mathrm{O}\right)_{2}\right] \mathrm{Cl}_{2}(1000 \mathrm{~mol}$ ppm, $0.28 \mathrm{mg}), 2^{\prime}$-iodo-[1,1'-biphenyl]-2-carboxamide, 6 (1 mol equiv, $0.31 \mathrm{mmol}, 100 \mathrm{mg})$, phenylboronic acid, $2 \mathrm{e}(1.5 \mathrm{~mol}$ equiv, $0.46 \mathrm{mmol}, 57 \mathrm{mg})$ and $\mathrm{K}_{3} \mathrm{PO}_{4}(3 \mathrm{~mol}$ equiv, $0.93 \mathrm{mmol}, 197$ $\mathrm{mg}$ ) were added to a reaction tube. The reaction tube was degassed under vacuum and refile with $\mathrm{N}_{2}$ under standard Schlack techniques ( 3 times). 1,4-dioxane $(2 \mathrm{~mL})$ was added and seal the reaction tube with teflon, followed by stirring at $115{ }^{\circ} \mathrm{C}$ (chemiStation) under nitrogen for $30 \mathrm{~h}$. After that the reaction mixture was diluted by EtOAc and extracted with $\mathrm{H}_{2} \mathrm{O}$. The organic layer was collected and dried over $\mathrm{Na}_{2} \mathrm{SO}_{4}$ and filtered. The solvent was evaporated under vacuum and crude mass was purified by column chromatography to give products 7 . 
White solid (97\%, $59 \mathrm{mg})$. m.p. $293^{\circ} \mathrm{C}-294^{\circ} \mathrm{C}$; ${ }^{1} \mathrm{H}-\mathrm{NMR}\left(500 \mathrm{MHz}, \mathrm{DMSO}-\mathrm{d}_{6}\right) \delta 11.66$ (brs, $\left.1 \mathrm{H}\right), 8.48(\mathrm{~d}, J=$ $8.0 \mathrm{~Hz}, 1 \mathrm{H}), 8.36(\mathrm{~d}, J=8.0 \mathrm{~Hz}, 1 \mathrm{H}), 8.29(\mathrm{~d}, J=8.0 \mathrm{~Hz}, 1 \mathrm{H}), 7.82(\mathrm{t}, J=7.4 \mathrm{~Hz}, 1 \mathrm{H}), 7.62(\mathrm{t}, J=7.4 \mathrm{~Hz}, 1 \mathrm{H})$,

$7.46(\mathrm{t}, J=7.4 \mathrm{~Hz}, 1 \mathrm{H}), 7.34(\mathrm{~d}, J=8.0 \mathrm{~Hz}, 1 \mathrm{H}), 7.23(\mathrm{t}, J=7.4 \mathrm{~Hz}, 1 \mathrm{H}) .{ }^{13} \mathrm{C}\left\{{ }^{1} \mathrm{H}\right\}-\mathrm{NMR}(125 \mathrm{MHz}, \mathrm{DMSO}-$

$\left.\mathrm{d}_{6}\right) \delta 161.4,137.1,134.8,133.4,130.1,128.5,128.0,126.2,123.8,123.2,122.8,118.1,116.6$.

\section{Synthesis of Biologically Active Molecules}

a)
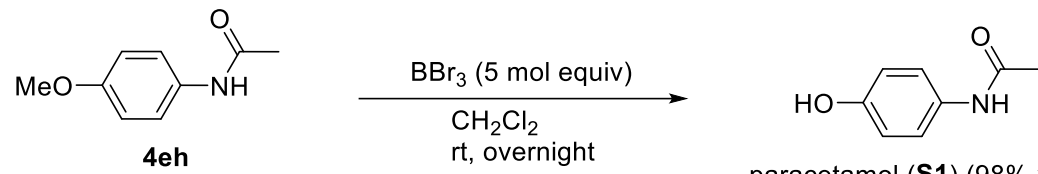

paracetamol (S1) (98\% yield)

b)

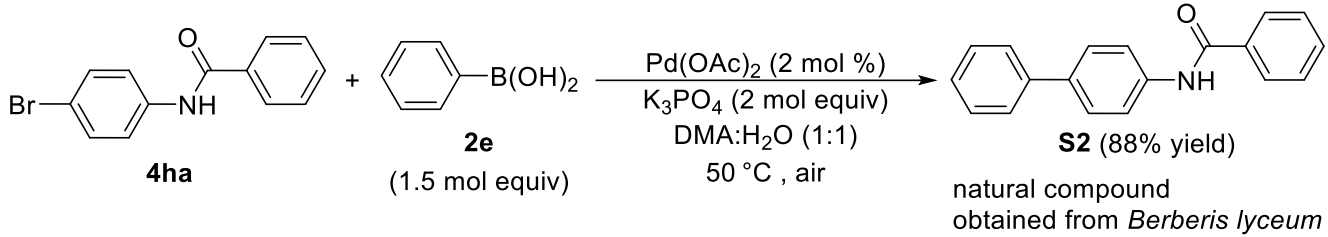

\section{Reaction Procedure for Paracetamol Synthesis}

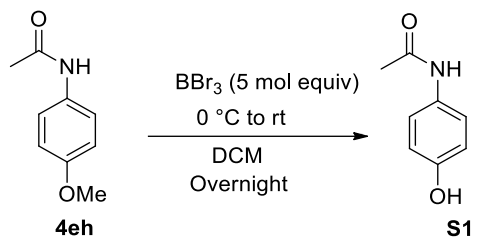

$\mathrm{N}$-(4-methoxyphenyl)acetamide, 4eh (1 mol equiv, $0.98 \mathrm{mmol}, 160 \mathrm{mg}$ ) was dissolved in $5 \mathrm{ml} \mathrm{DCM}$ in $25 \mathrm{ml}$ roundbottomed flask. The reaction flask was placed over an ice bath. After that $1 \mathrm{M}$ DCM solution of $\mathrm{BBr}_{3} \mathrm{was}_{\text {added dropwise }}$ to the reaction mixture and stirred for 1 hour. The ice bath was removed and the reaction was continued for overnight (14 h) at room temperature. All volatiles were evaporated under vacuum and the crude mass was purified by column chromatography with ethyl acetate as eluent to afford desired paracetamol S1. We examined the nickel contamination on paracetamol. To our delight nickel contamination was not detected by ICP-mass analysis.

$N$-(4-hydroxyphenyl)acetamide, paracetamol (S1, Ref. 32)<smiles>CCC(=O)Nc1ccc(O)cc1</smiles>

White solid (96\%, $141 \mathrm{mg})$. m.p. $155^{\circ} \mathrm{C}-156^{\circ} \mathrm{C} ;{ }^{1} \mathrm{H}-\mathrm{NMR}\left(500 \mathrm{MHz}\right.$, methanol- $\left.d_{3}\right) \delta 7.28(\mathrm{~d}, J=8.8 \mathrm{~Hz}$, $2 \mathrm{H}), 6.70(\mathrm{~d}, J=8.8 \mathrm{~Hz}, 2 \mathrm{H}), 2.06(\mathrm{~s}, 3 \mathrm{H}) .{ }^{13} \mathrm{C}\left\{{ }^{1} \mathrm{H}\right\}-\mathrm{NMR}\left(125 \mathrm{MHz}\right.$, methanol- $\left.d_{3}\right) \delta 170.1,153.9,130.4$,

$122.02,114.8,22.2$.

\section{Procedure for Natural Product S2 Synthesis:}
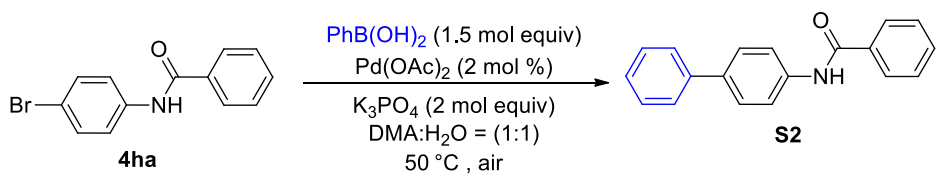

A mixture of $N$-(4-bromophenyl)benzamide, 4 ha ( $1 \mathrm{~mol}$ equiv, $1 \mathrm{mmol}, 296 \mathrm{mg}$ ), phenylboronic acid $2 \mathrm{e}$ ( $1.5 \mathrm{~mol}$ equiv, $183 \mathrm{mg}), \mathrm{Pd}(\mathrm{OAc})_{2}(2 \mathrm{~mol} \%, 4 \mathrm{mg})$ and $\mathrm{K}_{3} \mathrm{PO}_{4}(2 \mathrm{~mol}$ equiv, $424 \mathrm{mg})$ were taken in a reaction tube. In the reaction mixture, $1 \mathrm{ml} \mathrm{H}_{2} \mathrm{O}$ and $1 \mathrm{ml} \mathrm{DMA}$ was added at a time. The reaction mixture was placed over a chemiStation at $50{ }^{\circ} \mathrm{C}$ for 
overnight. After the reaction, the reaction mixture was diluted by EtOAc and extracted with $\mathrm{H}_{2} \mathrm{O}$. The organic layer was collected and dried over $\mathrm{Na}_{2} \mathrm{SO}_{4}$ and filtered. The organic volatiles were evaporated under vacuum and crude mass was purified by column chromatography to afford $\mathbf{S 2}$.

$N$-([1,1'-biphenyl]-4-yl)benzamide (S2, Ref. 33)

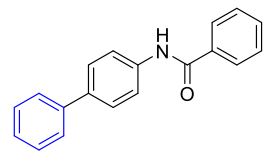

White solid (88\%, $240 \mathrm{mg})$. m.p. $234^{\circ} \mathrm{C}-235^{\circ} \mathrm{C} ;{ }^{1} \mathrm{H}-\mathrm{NMR}\left(500 \mathrm{MHz}, \mathrm{CDCl}_{3}\right) \delta 7.85-7.90(\mathrm{~m}, 3 \mathrm{H})$, $7.72(\mathrm{dd}, J=8.6,2.3 \mathrm{~Hz}, 2 \mathrm{H}), 7.49-7.63(\mathrm{~m}, 7 \mathrm{H}), 7.42-7.45(\mathrm{~m}, 2 \mathrm{H}), 7.32-7.35(\mathrm{~m}, 1 \mathrm{H}) .{ }^{13} \mathrm{C}\left\{{ }^{1} \mathrm{H}\right\}-$ $\operatorname{NMR}\left(125 \mathrm{MHz}, \mathrm{CDCl}_{3}\right) \delta 165.7,140.6,137.5,137.3,135.0,132.0,129.0,128.9,127.8,127.3,127.1$,

127.0, 120.5.

\section{Procedure for Large Scale Reaction and ICP-Mass Analysis}

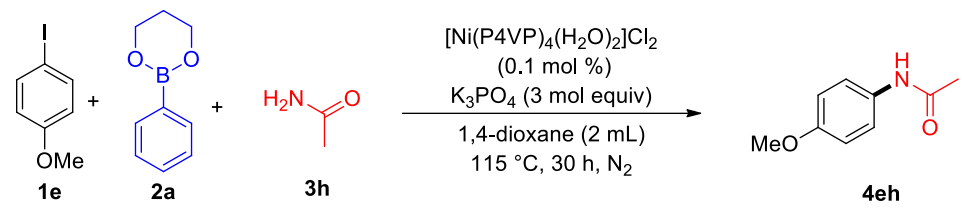

A mixture of $\left[\mathrm{Ni}(\mathrm{P} 4 \mathrm{VP})_{4}\left(\mathrm{H}_{2} \mathrm{O}\right)_{2}\right] \mathrm{Cl}_{2}(1000 \mathrm{~mol} \mathrm{ppm}, 7.7 \mathrm{mg})$, aryl iodide, $\mathbf{1}$ (1 mol equiv, $\left.8.5 \mathrm{mmol}, 2 \mathrm{~g}\right)$, phenylboronic ester, 2 (1.5 mol equiv, 12.7 mmol, $2 \mathrm{~g}$ ), acetamide $3 \mathrm{~h}$ ( 3 mol equiv, $26 \mathrm{mmol}, 1.5 \mathrm{~g})$ and $\mathrm{K}_{3} \mathrm{PO}_{4}(3 \mathrm{~mol}$ equiv, $26 \mathrm{mmol}$, $5.5 \mathrm{~g}$ ) were added to a round bottomed flask. The flask was degassed under vacuum and refile with $\mathrm{N}_{2}$ under standard Schlack techniques (3 times). 1,4-dioxane (30 mL) was added and seal the reaction flask with teflon, followed by stirring at reflux (oil bath temperature $115^{\circ} \mathrm{C}$ ) under nitrogen for $30 \mathrm{~h}$. After the reaction, the reaction mixture was diluted by EtOAc and extracted with $\mathrm{H}_{2} \mathrm{O}$. The organic layer was collected and dried over $\mathrm{Na}_{2} \mathrm{SO}_{4}$ and filtered. The solvent was evaporated under vacuum and crude mass was purified by column chromatography (hexane/EtOAc $=5 / 1)$ to give products 4. The amount of metal contamination on the product was determined by ICP-Mass analysis. Only 1.7 ppm nickel contamination was observed.

\section{Reaction Mechanism}

\subsection{Identification of Reaction Intermediates:}




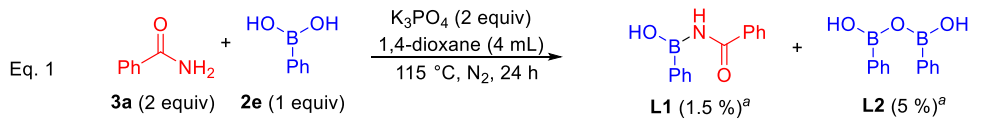

$$
\begin{aligned}
& \text { Eq. } 2 \text { 3a (2 equiv) }+2 \mathbf{e} \text { (1 equiv) } \frac{\text { 1,4-dioxane }(4 \mathrm{~mL})}{115^{\circ} \mathrm{C}, \mathrm{N}_{2}, 24 \mathrm{~h}} \quad \mathbf{L 1}(20 \%)^{\mathrm{a}}
\end{aligned}
$$

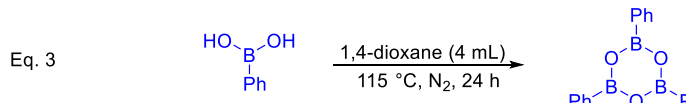

$$
\begin{aligned}
& \text { 2e } \quad 115^{\circ} \mathrm{C}, \mathrm{N}_{2}, 24 \mathrm{~h} \quad \mathrm{Ph}^{-\mathrm{B}_{-}} \mathrm{O}^{-\mathrm{B}_{-} \mathrm{Ph}}
\end{aligned}
$$

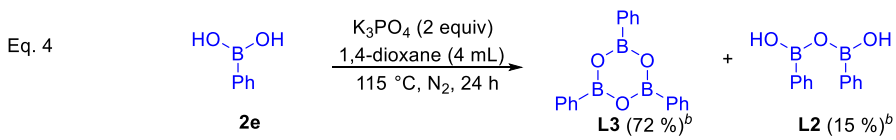

$$
\begin{aligned}
& \text { Eq.5 }
\end{aligned}
$$

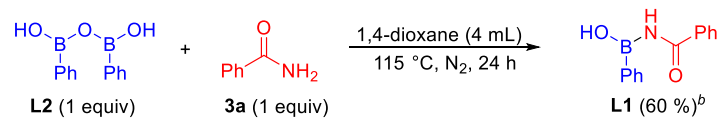

$$
\begin{aligned}
& \text { Eq. } 6
\end{aligned}
$$

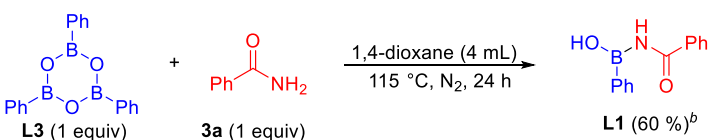

$$
\begin{aligned}
& \mathrm{L1} \text { (1 equiv) + }
\end{aligned}
$$

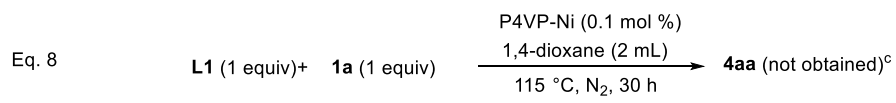

\section{$N$-(hydroxy(phenyl)boraneyl)benzamide, L1}

$\mathrm{HO}_{\mathrm{B}^{\mathrm{B}^{-}}} \mathrm{M}_{\mathrm{Ph}}^{\mathrm{H}} \mathrm{O}^{\mathrm{Ph}}$

White solid (20\%, $44 \mathrm{mg}) .{ }^{1} \mathrm{H}-\mathrm{NMR}\left(500 \mathrm{MHz}, \mathrm{CDCl}_{3}\right) \delta 8.87$ (brs, 1H), 8.15-8.32 (m, 1H), 7.68-7.98 (m, $2 \mathrm{H})$, 7.35-7.64 (m, 5H), 7.23-2.28 (m, 2H), 4.64 (brs, $1 \mathrm{H}) .{ }^{13} \mathrm{C}\left\{{ }^{1} \mathrm{H}\right\}-\mathrm{NMR}\left(125 \mathrm{MHz}, \mathrm{CDCl}_{3}\right) \delta 166.4$,

135.7, 133.7, 133.4, 133.3, 132.8, 129.0, 128.1, 128.0. ESI-MS: calcd for $\mathrm{C}_{13} \mathrm{H}_{12} \mathrm{BNO}_{2}: \mathrm{m} / \mathrm{z} 248.0853$ $\left([\mathrm{M}+\mathrm{Na}]^{+}\right)$, found: $m / z 248.0886$.

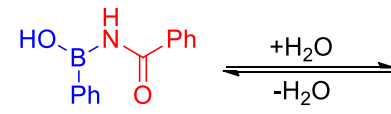

L1

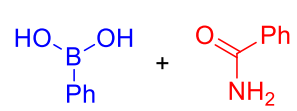

$3 \mathbf{a}$ 


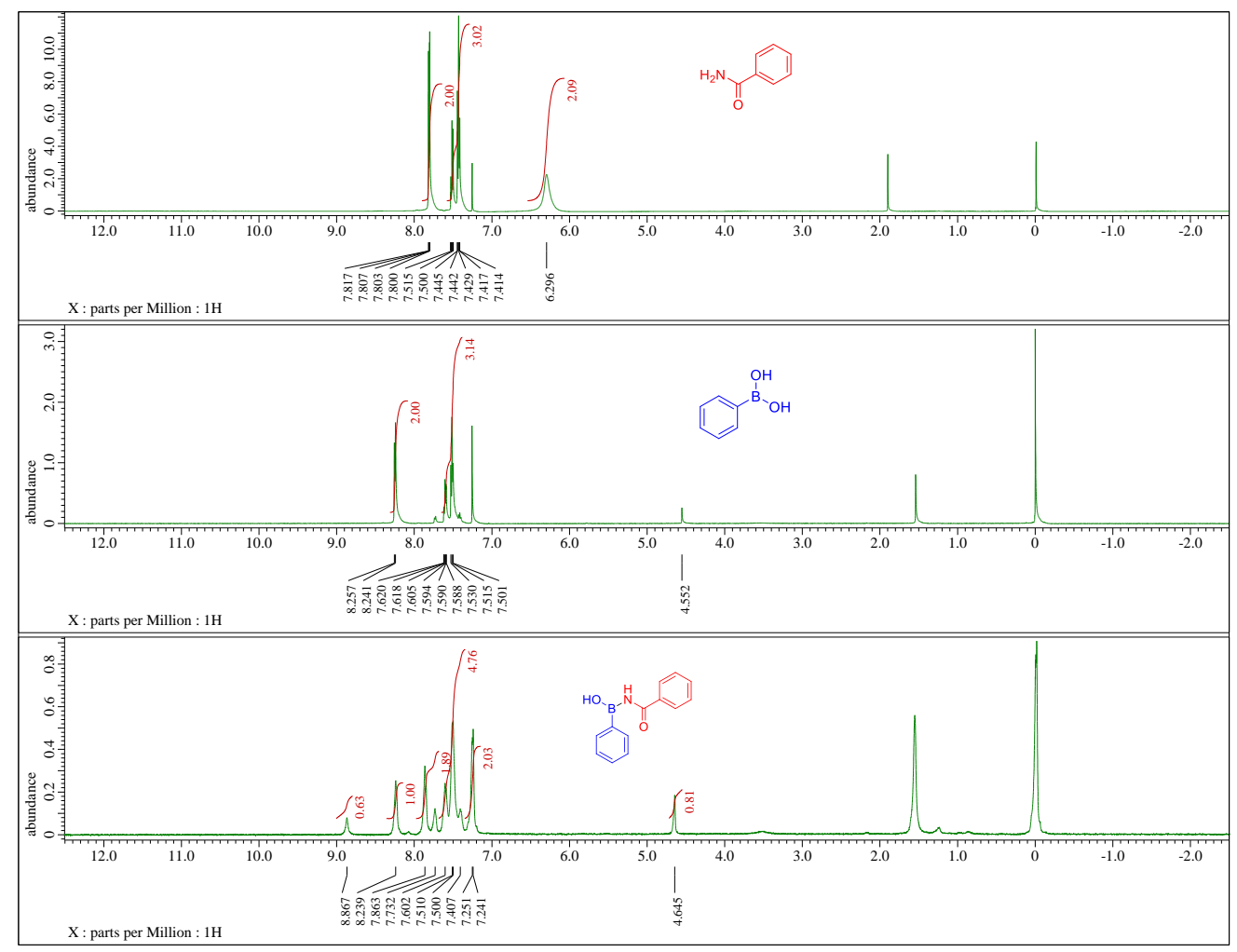

Figure S4-1. ${ }^{1} \mathrm{H}$ NMR spectrum of Benzamide (top), Phenylboronic acid (middle), and $\mathbf{L 1}\left(500 \mathrm{MHz}, \mathrm{CDCl}_{3}\right.$ )

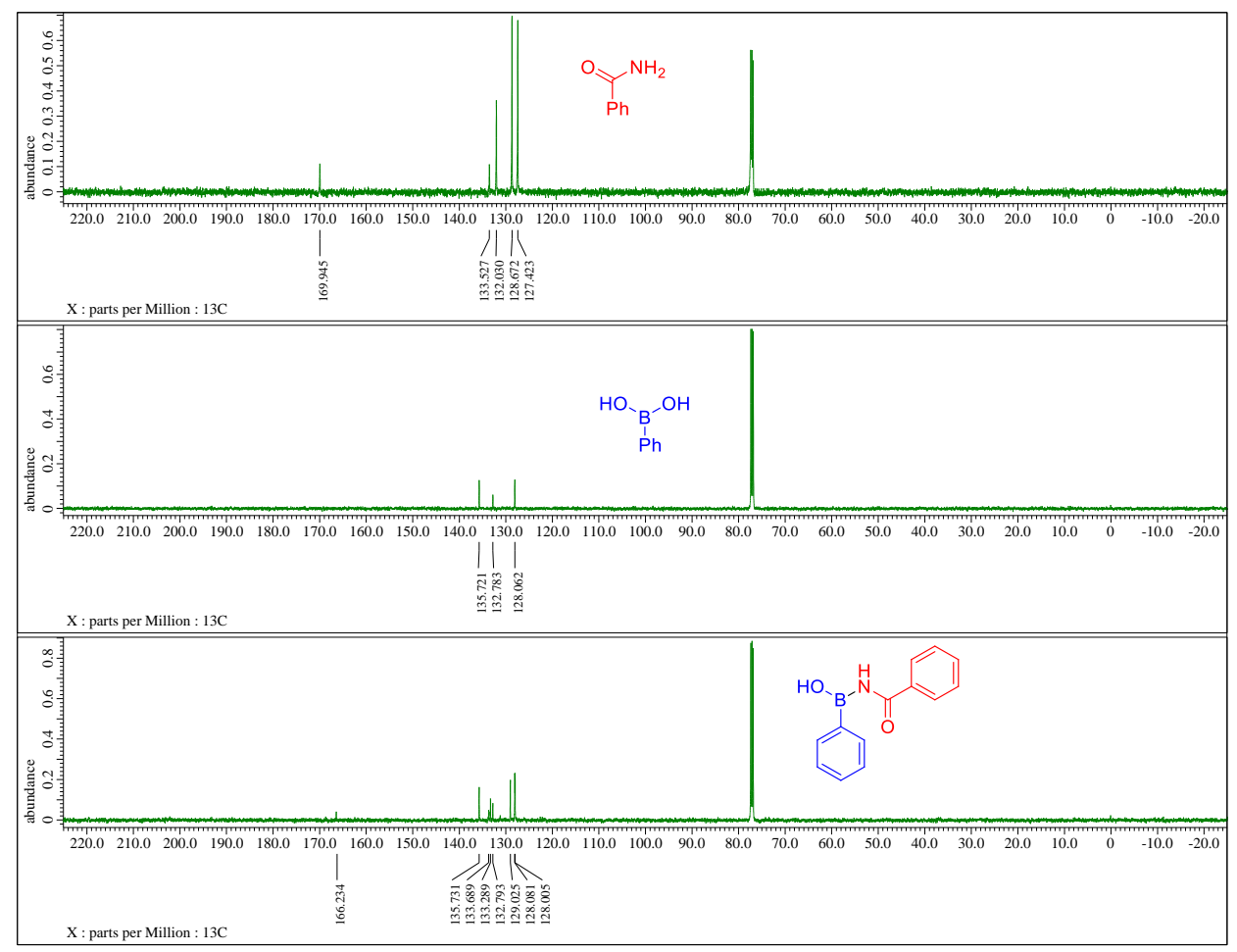

Figure S4-2. ${ }^{13} \mathrm{C}$ NMR spectrum of Benzamide (top), Phenylboronic acid (middle), and L1 (125 MHz, $\left.\mathrm{CDCl}_{3}\right)$

From the ${ }^{1} \mathrm{H}-\mathrm{NMR}$ we clearly see two new broad signals at 4.65 and $8.87 \mathrm{ppm}$ which might correspond to O-H and N-H of $\mathbf{L 1}$. The compound $\mathbf{L 1}$ is very unstable especially in solution and undergo decomposition to provide 
benzamide and phenylboronic acid. ${ }^{13} \mathrm{C}$ NMR suggest the presence of $\mathrm{CONH}$ bond by providing signal at 166.2 ppm. Since the ${ }^{1} \mathrm{H}$-NMR signals are broad in nature (might be due to equilibrium) we are not able to confirm the structure but here we proposed $\mathbf{L} \mathbf{1}$ as the putative reaction intermediate. We tried to obtain single crystals of $\mathbf{L 1}$ unfortunately, we ended up with phenylboronic acid $\mathbf{2 e}$, phenylboronic acid trimer (triphenylboroxine) L3, and benzamide 3a. DFT calculation showed L1 as the structure not the L1'.

DFT Calculation Details: Since from the NMR studies it is not clear whether the L1 is connected via NH or via $\mathrm{O}(\mathbf{L 1}$ '), We performed single point calculations on the optimized structure of L1_BN (amide form, N-bound) and L1_BO (imidic acid form, O-bound) at M06-2X/6-31G(d) level of theory with and without SMD solvation model for 1,4dioxane. In either case, N-bound L1_BN is more thermodynamically favourable than O-bound L1_BO (G(L1_BN)$\mathrm{G}\left(\mathrm{L} 1 \_\mathrm{BO}\right)=-26.7 \mathrm{~kJ} / \mathrm{mol}$ in gas phase; $\mathrm{G}\left(\mathrm{L} 1 \_\mathrm{BN}\right)-\mathrm{G}\left(\mathrm{L} 1 \_\mathrm{BO}\right)=-32.3 \mathrm{~kJ} / \mathrm{mol}$ in 1,4-dioxane), suggesting that L1_BN is the predominant structure of $\mathrm{L} 1$.

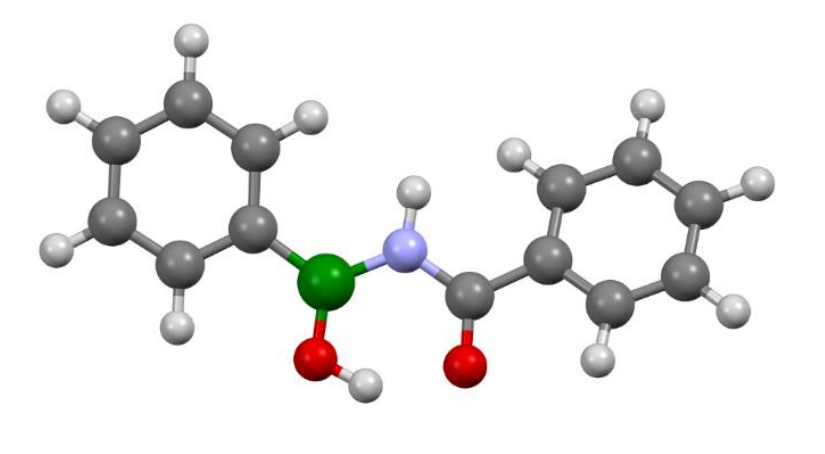

B-N intermediate (L1)

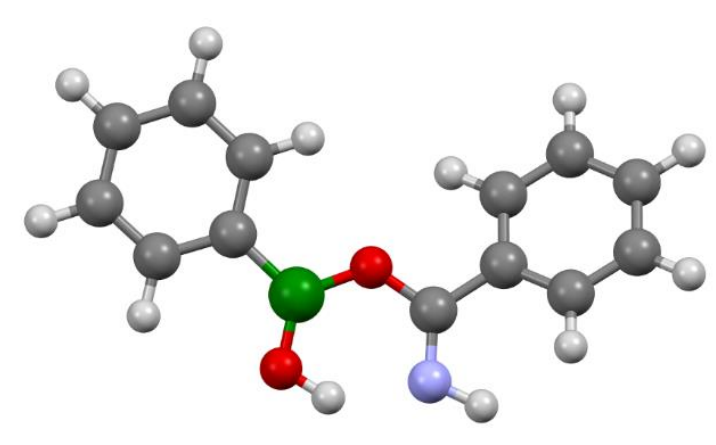

B-O intermediate (L1')

$\begin{array}{llllll} & & \mathrm{G}(\mathrm{au}) & \Delta \mathrm{G}(\mathrm{au}) & \Delta \mathrm{G}(\mathrm{kcal} / \mathrm{mol}) & \Delta \mathrm{G}(\mathrm{kJ} / \mathrm{mol}) \\ \mathrm{B}-\mathrm{N}(\mathbf{L 1}) & \text { gas } & -732.282 & - & - & - \\ \mathrm{B}-\mathrm{O}\left(\mathbf{L 1}{ }^{\prime}\right) & \text { gas } & -732.2718 & 0.0101737 & 6.4 & 26.7 \\ \text { B-N (L1) } & \text { dioxane } & -732.2952 & - & - & - \\ \text { BO (L1') } & \text { dioxane } & -732.2829 & 0.0123168 & 7.7 & 32.3\end{array}$

\section{diphenyldiboronic acid, $\mathrm{L} 2$}

$\mathrm{HO}_{\mathrm{B}^{-}} \mathrm{O}_{\mathrm{B}^{-}} \mathrm{OH} \quad$ White solid $(4.9 \%, 11 \mathrm{mg}) .{ }^{1} \mathrm{H}-\mathrm{NMR}\left(500 \mathrm{MHz}, \mathrm{CDCl}_{3}\right) \delta$ 7.80-7.82 (m, 2H), 7.44-7.53 (m, 3H), 5.85 (s, $\stackrel{\mathrm{Ph}}{\mathrm{Ph}} \quad 1 \mathrm{H}) .{ }^{13} \mathrm{C}\left\{{ }^{1} \mathrm{H}\right\}-\mathrm{NMR}\left(125 \mathrm{MHz}, \mathrm{CDCl}_{3}\right) \delta 134.8,131.2,128.0$. HRMS-MS: data not obtained (unstable)

\subsection{Comparison Between Phosphate-Borate-Amide Complex vs Direct Deprotonation (DFT)}




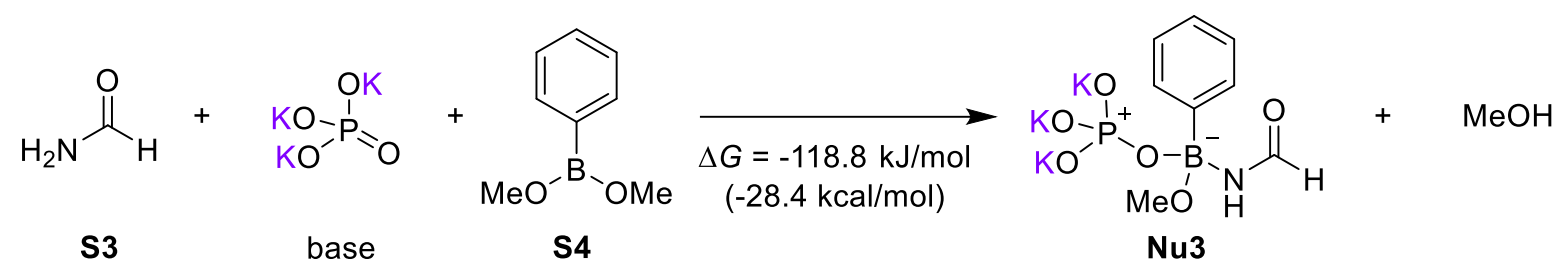<smiles>[R10]O[R20]#N</smiles>

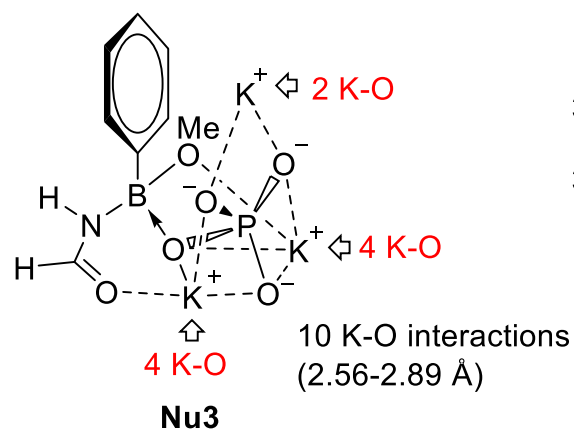<smiles>CN[Pb](OC)(O[Na])c1ccccc1</smiles>

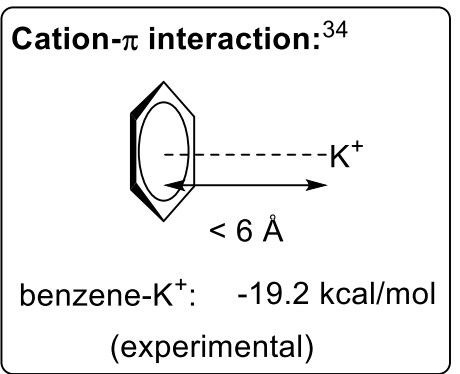

10.4. Conclusion Based on the Computational Analysis and Experimental Observation

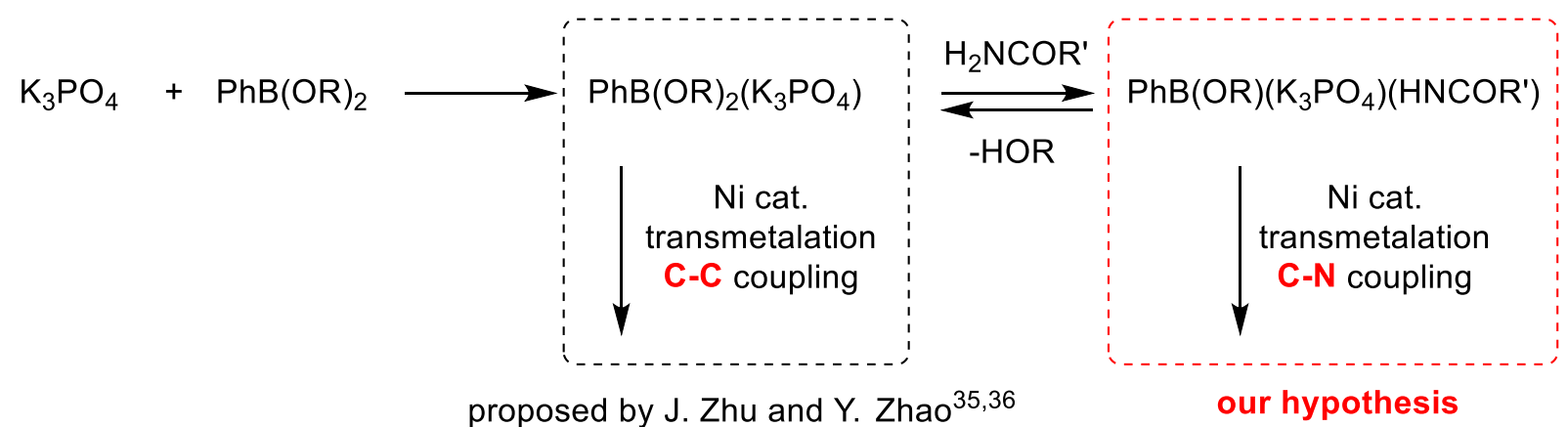

10.5. Proposed Reaction Mechanism for C-N Bond Forming Amidation Reaction 


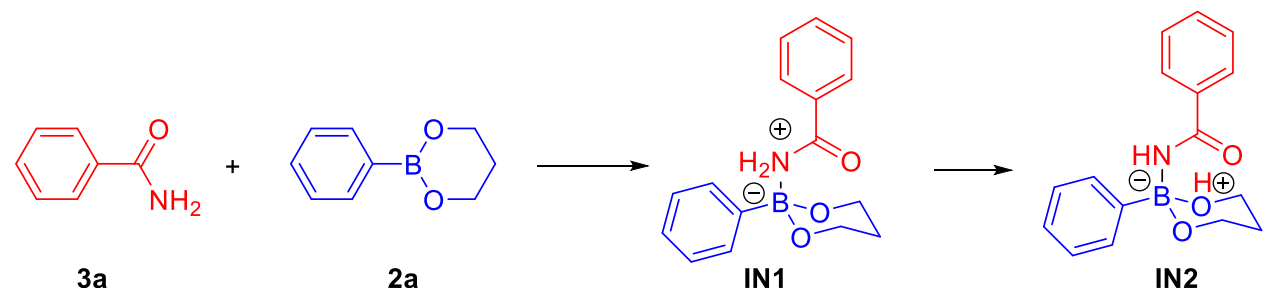


A mixture of $\left[\mathrm{Ni}(\mathrm{P} 4 \mathrm{VP})_{4}\left(\mathrm{H}_{2} \mathrm{O}\right)_{2}\right] \mathrm{Cl}_{2}(\mathrm{X}$ mol \%), aryl iodide, $\mathbf{1 a}(1 \mathrm{~mol}$ equiv, $0.5 \mathrm{mmol})$, benzamide, $3 \mathbf{a}$ (3 mol equiv, 1.5 equiv), arylboronic ester, $2 \mathrm{a}(1.5 \mathrm{~mol}$ equiv, $0.75 \mathrm{mmol})$, and $\mathrm{K}_{3} \mathrm{PO}_{4}(3 \mathrm{~mol}$ equiv, $1.5 \mathrm{mmol}, 318 \mathrm{mg})$ were added to a reaction tube. The reaction tube was degassed under vacuum and refile with $\mathrm{N}_{2}$ under standard Schlack techniques (3 times). Mesitylene (100 $\mu \mathrm{L})$ and 1,4-dioxane $(2 \mathrm{~mL})$ was added and seal the reaction tube with teflon, followed by stirring at $115{ }^{\circ} \mathrm{C}$ (chemiStation) for $4 \mathrm{~h}$. After $3 \mathrm{~h}$ the reaction tube was opened inside a glove box to take a small amount of reaction mixture and the reaction tube was resealed and further placed over the chemiStation for an additional hour. The collected reaction mixture was filtered and the filtrate was analyzed by GC. After $4 \mathrm{~h}$ the reaction mixture opened and filtered. The filtrate was analyzed by GC.

\begin{tabular}{|c|c|c|c|c|c|c|}
\hline \multirow[t]{2}{*}[\text{Cat}]{} & \multicolumn{2}{|c|}{$[\mathbf{1 a}] \times 10^{6}$} & \multirow{2}{*}{$\begin{array}{c}\mathrm{v}=\text { initial rate } \\
\times 10^{6}\end{array}$} & \multirow[t]{2}{*}{$\operatorname{lnv}$} & \multirow[t]{2}{*}[\mathbf{cat}]{$^{*}=10 \times[\mathbf{c a t}]$} & \multirow[t]{2}{*}{$\ln [\text { cat }]^{*}$} \\
\hline & $3 \mathrm{~h}$ & $4 \mathrm{~h}$ & & & & \\
\hline $0.1 \mathrm{~mol} \%$ & 0.4769 & 0.5042 & 0.0273 & -3.6 & 1 & 0 \\
\hline $0.2 \mathrm{~mol} \%$ & 0.4600 & 0.4873 & 0.0273 & -3.6 & 2 & 0.69 \\
\hline $0.3 \mathrm{~mol} \%$ & 0.4988 & 0.5322 & 0.0334 & -3.4 & 3 & 1.1 \\
\hline $0.4 \mathrm{~mol} \%$ & 0.5169 & 0.5503 & 0.0334 & -3.4 & 4 & 1.4 \\
\hline $0.5 \mathrm{~mol} \%$ & 0.5585 & 0.5954 & 0.0369 & -3.3 & 5 & 1.6 \\
\hline
\end{tabular}

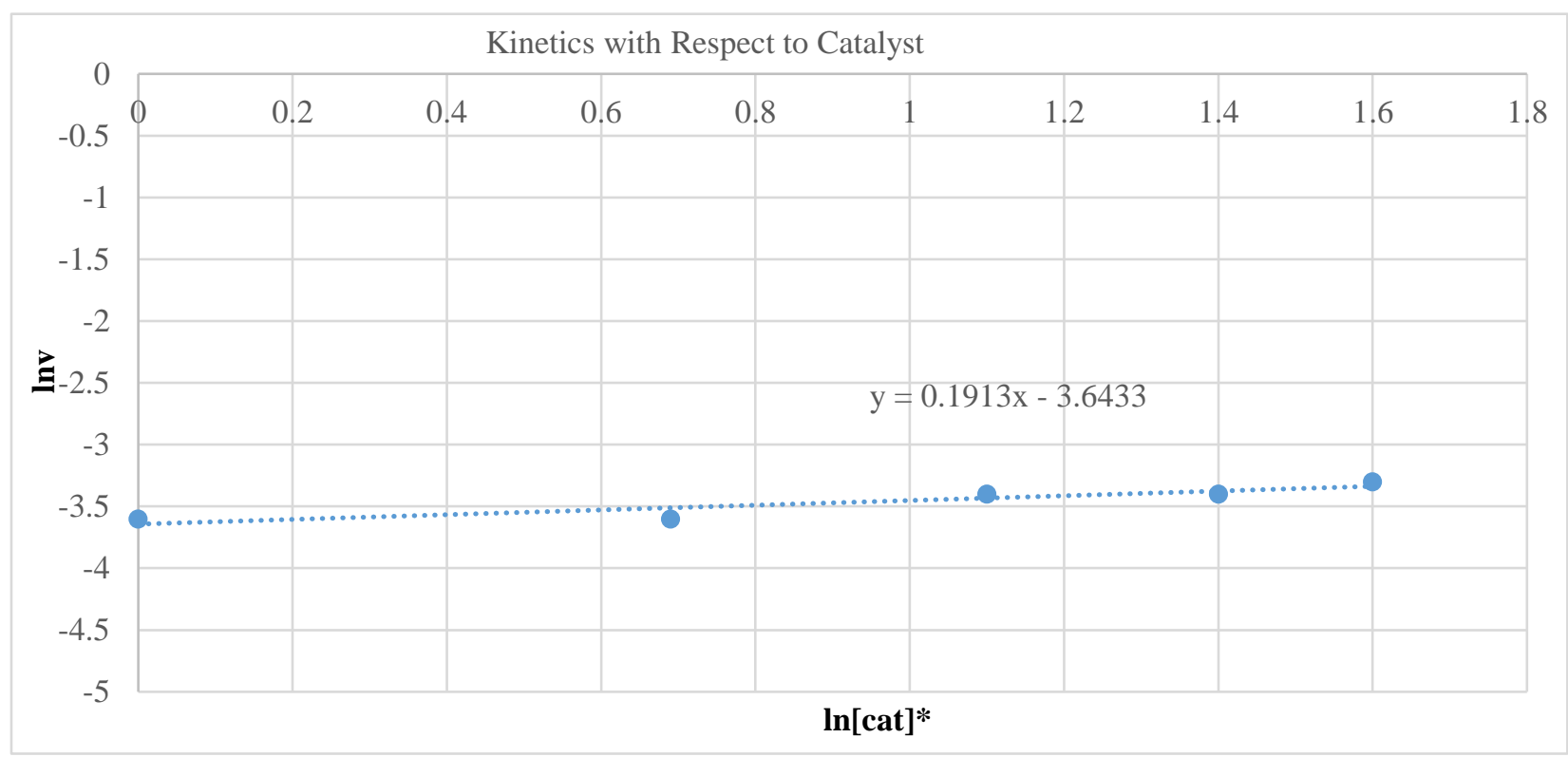

Figure S5: Graphical plot lnv vs $\ln [\text { Cat }]^{*}$

If $\mathrm{v}$ is the rate of reaction, $\mathrm{n}$ is the order of the reaction, [Cat $]^{*}$ is the concentration of the catalyst then

$$
\begin{gathered}
\text { Rate }=\mathrm{v}=\mathrm{k}[\mathbf{C a t}]^{* \mathrm{n}} \text { where } \mathrm{k} \text { is the rate constant. } \\
\operatorname{lnv}=\mathrm{n} \ln [\mathbf{C a t}]^{*}+\mathrm{C} \text { where } \mathrm{C} \text { is a constant }
\end{gathered}
$$




\subsection{Kinetics with Respect to Aryl Iodide for C-N Coupling}

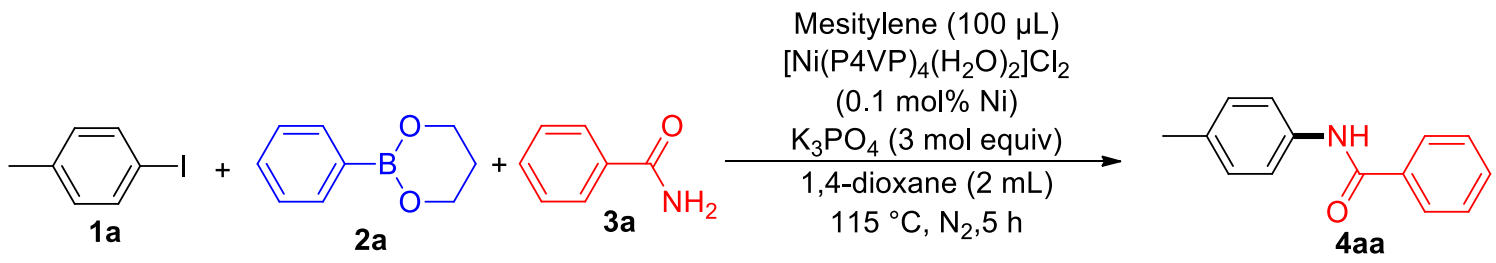

A mixture of $\left[\mathrm{Ni}(\mathrm{P} 4 \mathrm{VP})_{4}\left(\mathrm{H}_{2} \mathrm{O}\right)_{2}\right] \mathrm{Cl}_{2}(0.1 \mathrm{~mol} \%)$, aryl iodide, $1 \mathbf{a}(\mathrm{X}$ mol equiv, $0.5 \mathrm{mmol})$, benzamide, $3 \mathbf{a}$ (3 mol equiv, 1.5 equiv), arylboronic ester, $2 \mathbf{a}(1.5 \mathrm{~mol}$ equiv, $0.75 \mathrm{mmol})$, and $\mathrm{K}_{3} \mathrm{PO}_{4}(3 \mathrm{~mol}$ equiv, $1.5 \mathrm{mmol}, 318 \mathrm{mg})$ were added to a reaction tube. The reaction tube was degassed under vacuum and refile with $\mathrm{N}_{2}$ under standard Schlack techniques (3 times). Mesitylene $(100 \mu \mathrm{L})$ and 1,4-dioxane $(2 \mathrm{~mL})$ was added and seal the reaction tube with teflon, followed by stirring at $115^{\circ} \mathrm{C}$ (chemiStation) for $5 \mathrm{~h}$. After $4 \mathrm{~h}$ the reaction tube was opened inside a glove box to take a small amount of reaction mixture and the reaction tube was resealed and further placed over the chemiStation for an additional hour. The collected reaction mixture was filtered and the filtrate was analyzed by GC. After $5 \mathrm{~h}$ the reaction mixture opened and filtered. The filtrate was analyzed by GC.

\begin{tabular}{|c|c|c|c|c|c|}
\hline \multirow[t]{2}{*}{ 1a } & \multicolumn{2}{|c|}{$[\mathbf{1 a}] \times 10^{6}$} & \multirow[t]{2}{*}{$\mathrm{v}=$ initial rate $\times 10^{6}$} & \multirow[t]{2}{*}{$\operatorname{lnv}$} & \multirow[t]{2}{*}{$\ln [\mathbf{1 a}]$} \\
\hline & $4 \mathrm{~h}$ & $5 \mathrm{~h}$ & & & \\
\hline 1 equiv & 0.2401 & 0.2171 & 0.023 & -3.8 & 0 \\
\hline 2 equiv & 0.6350 & 0.6099 & 0.025 & -3.7 & 0.69 \\
\hline 3 equiv & 1.0603 & 1.0323 & 0.028 & -3.6 & 1.1 \\
\hline 4 equiv & 1.5107 & 1.4827 & 0.028 & -3.6 & 1.4 \\
\hline 5 equiv & 2.0298 & 2.0019 & 0.028 & -3.6 & 1.6 \\
\hline
\end{tabular}




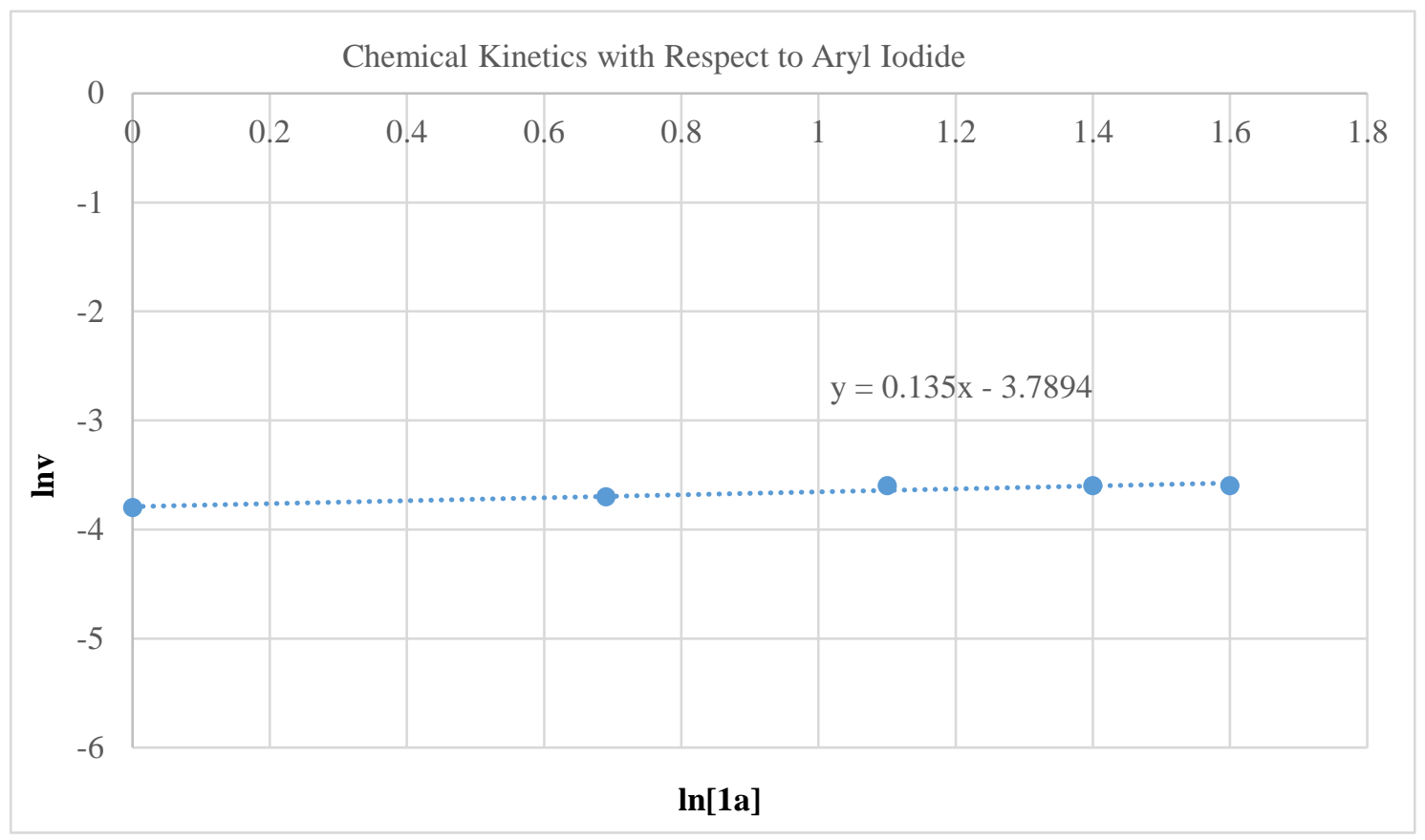

Figure S6. Graphical plot of $\operatorname{lnv}$ vs $\ln [\mathbf{1 a}]$

If $\mathrm{v}$ is the rate of reaction, $\mathrm{n}$ is the order of the reaction, $[\mathbf{1 a}]$ is the concentration of the $\mathbf{1 a}$ then

Rate $=\mathrm{v}=\mathrm{k}[\mathbf{1} \mathbf{a}]^{\mathrm{n}}$ where $\mathrm{k}$ is the rate constant.

$\operatorname{lnv}=n \ln [\mathbf{1} \mathbf{a}]+C$ where $C$ is a constant

therefore, the order of the reaction $n=0.135 \approx 0$

\subsection{Kinetics with Respect to Phenylboronic Ester for C-N Coupling Reaction}

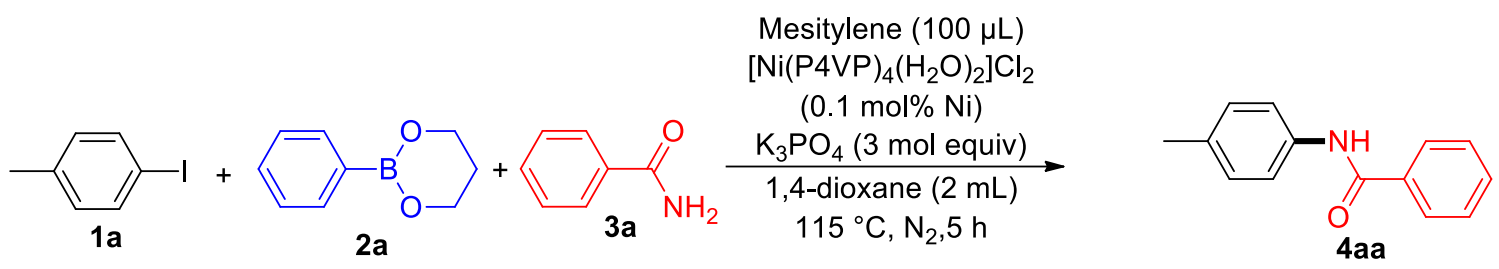

A mixture of $\left[\mathrm{Ni}(\mathrm{P} 4 \mathrm{VP})_{4}\left(\mathrm{H}_{2} \mathrm{O}\right)_{2}\right] \mathrm{Cl}_{2}(0.1 \mathrm{~mol} \%)$, aryl iodide, $\mathbf{1 a}(1 \mathrm{~mol}$ equiv, $0.5 \mathrm{mmol})$, benzamide, $3 \mathbf{a}$ (3 mol equiv, 1.5 equiv), arylboronic ester, $2 \mathrm{a}$ ( $\mathrm{X}$ mol equiv), and $\mathrm{K}_{3} \mathrm{PO}_{4}$ (3 mol equiv, $1.5 \mathrm{mmol}, 318 \mathrm{mg}$ ) were added to a reaction tube. The reaction tube was degassed under vacuum and refile with $\mathrm{N}_{2}$ under standard Schlack techniques (3 times). Mesitylene $(100 \mu \mathrm{L})$ and 1,4-dioxane $(2 \mathrm{~mL})$ was added and seal the reaction tube with teflon, followed by stirring at $115^{\circ} \mathrm{C}$ (chemiStation) for $5 \mathrm{~h}$. After $4 \mathrm{~h}$ the reaction tube was opened inside a glove box to take a small amount of reaction mixture and the reaction tube was resealed and further placed over the chemiStation for an additional hour. The collected reaction mixture was filtered and the filtrate was analyzed by GC. After $5 \mathrm{~h}$ the reaction mixture was opened and filtered. The filtrate was analyzed by GC for $5 \mathrm{~h}$ data.

\begin{tabular}{cccccc}
\hline 2a & \multicolumn{2}{c}{$[\mathbf{4 a a}] \times 10^{6}$} & $\mathrm{v}=$ initial rate $\times 10^{6}$ & $\ln$ & $\ln [\mathbf{2 a}]$ \\
& $4 \mathrm{~h}$ & $5 \mathrm{~h}$ & & & \\
\hline 1 equiv & 0.2371 & 0.2481 & 0.0111 & -4.5 & 0
\end{tabular}




\begin{tabular}{lccccc}
2 equiv & 0.5395 & 0.5577 & 0.0183 & -4.0 & 0.69 \\
3 equiv & 0.4849 & 0.5122 & 0.0273 & -3.6 & 1.1 \\
4 equiv & 0.6224 & 0.6632 & 0.0408 & -3.2 & 1.4 \\
5 equiv & 0.6452 & 0.6971 & 0.0519 & -3.0 & 1.6 \\
\hline
\end{tabular}

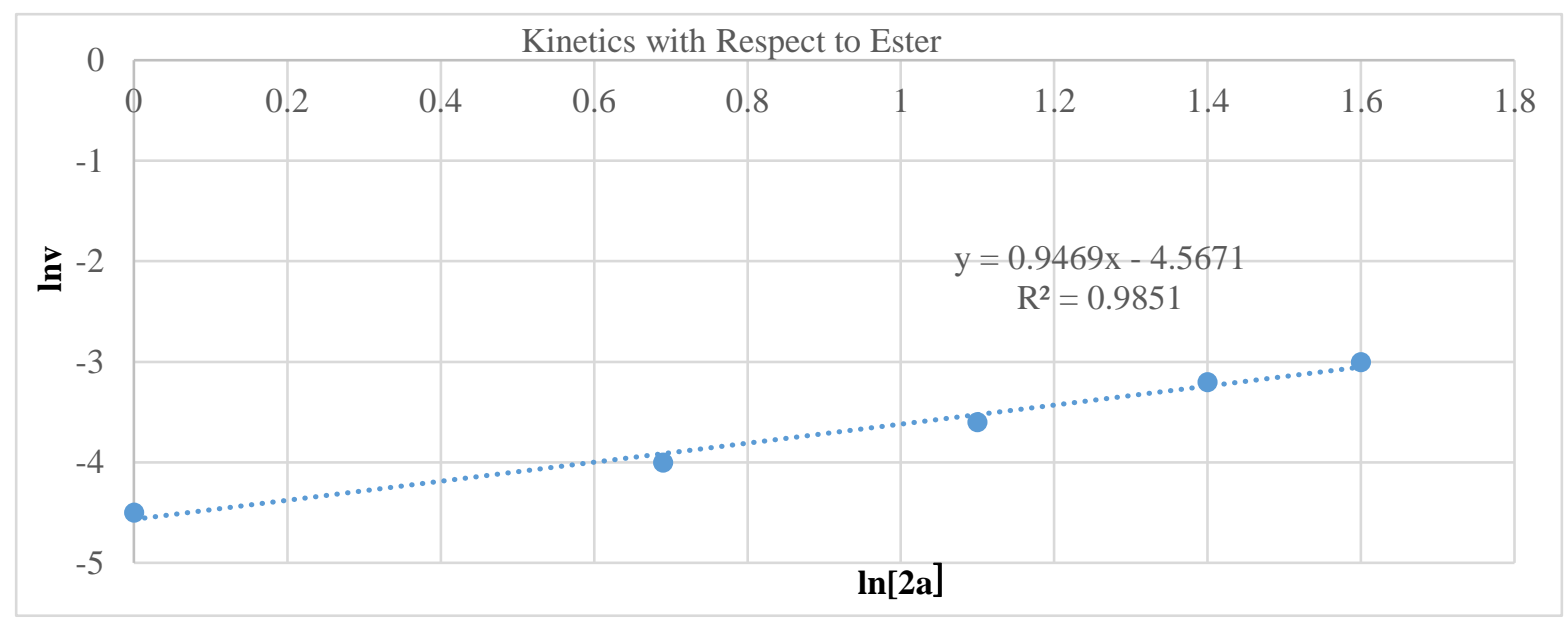

Figure S7. Graphical plot of $\operatorname{lnv}$ vs $\ln [\mathbf{2 a}]$

$\operatorname{lnv}=n \ln [\mathbf{2} \mathbf{a}]+\mathrm{C}$ where $\mathrm{n}$ is the order of the reaction and $\mathrm{C}$ is a constant.

Observed $\mathrm{n}=0.9469 \approx 1.0$

Therefore, the observed rate of reaction is 1 with respect to the phenylboronic ester

\subsection{Kinetics with Respect to Benzamide for C-N Coupling Reaction}

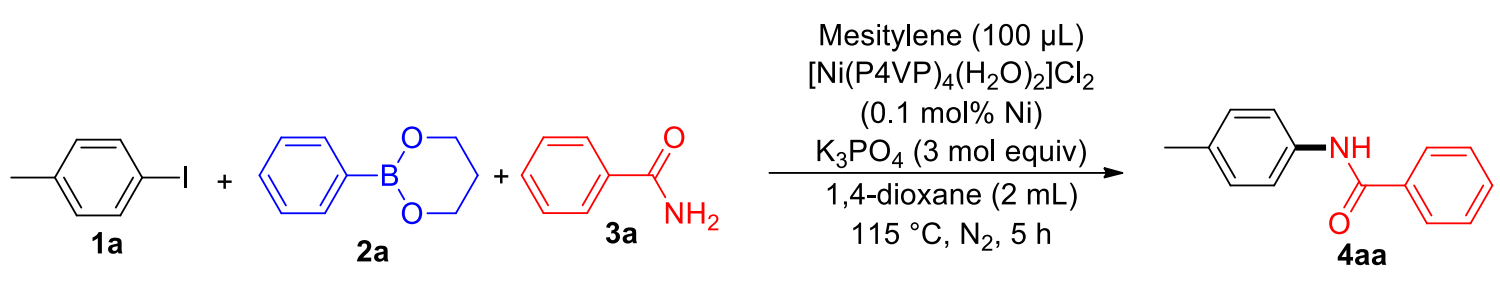

A mixture of $\left[\mathrm{Ni}(\mathrm{P} 4 \mathrm{VP})_{4}\left(\mathrm{H}_{2} \mathrm{O}\right)_{2}\right] \mathrm{Cl}_{2}(0.1 \mathrm{~mol} \%)$, aryl iodide, $1 \mathbf{a}(1 \mathrm{~mol}$ equiv, $0.5 \mathrm{mmol})$, benzamide, 3a (X mol equiv), arylboronic ester, $2 \mathbf{a}(1.5 \mathrm{~mol}$ equiv, $0.75 \mathrm{mmol})$, and $\mathrm{K}_{3} \mathrm{PO}_{4}(3 \mathrm{~mol}$ equiv, $1.5 \mathrm{mmol}, 318 \mathrm{mg})$ were added to a reaction tube. The reaction tube was degassed under vacuum and refile with $\mathrm{N}_{2}$ under standard Schlack techniques (3 times). Mesitylene (100 $\mu \mathrm{L})$ and 1,4-dioxane $(2 \mathrm{~mL})$ was added and seal the reaction tube with teflon, followed by stirring at $115{ }^{\circ} \mathrm{C}$ (chemiStation) for $5 \mathrm{~h}$. After $4 \mathrm{~h}$ the reaction tube was opened inside a glove box to take a small amount of reaction mixture and the reaction tube was resealed and further placed over the chemiStation for an additional hour. The collected reaction mixture was filtered and the filtrate was analyzed by GC. After $5 \mathrm{~h}$ the reaction mixture was opened and filtered. The filtrate was analyzed by GC for $5 \mathrm{~h}$ data. 


\begin{tabular}{cccccc}
\hline 3a & \multicolumn{2}{c}{$[\mathbf{3 a}] \times 10^{6}$} & $\mathrm{v}=$ initial rate $\times 10^{6}$ & $\operatorname{lnv}$ & $\ln [\mathbf{3 a}]$ \\
& $4 \mathrm{~h}$ & $5 \mathrm{~h}$ & & & \\
\hline 1 equiv & 0.5227 & 0.5157 & 0.0070 & -4.9 & 0 \\
& & & & & \\
2 equiv & 0.8706 & 0.8523 & 0.0183 & -4.0 & 0.69 \\
& & & & & 1.1 \\
3 equiv & 1.4798 & 1.4525 & 0.0273 & -3.6 & 1.4 \\
& & & & -2.9 & 1.6 \\
\hline equiv & 2.5831 & 2.5281 & 0.0550 & -2.6 & \\
\hline
\end{tabular}

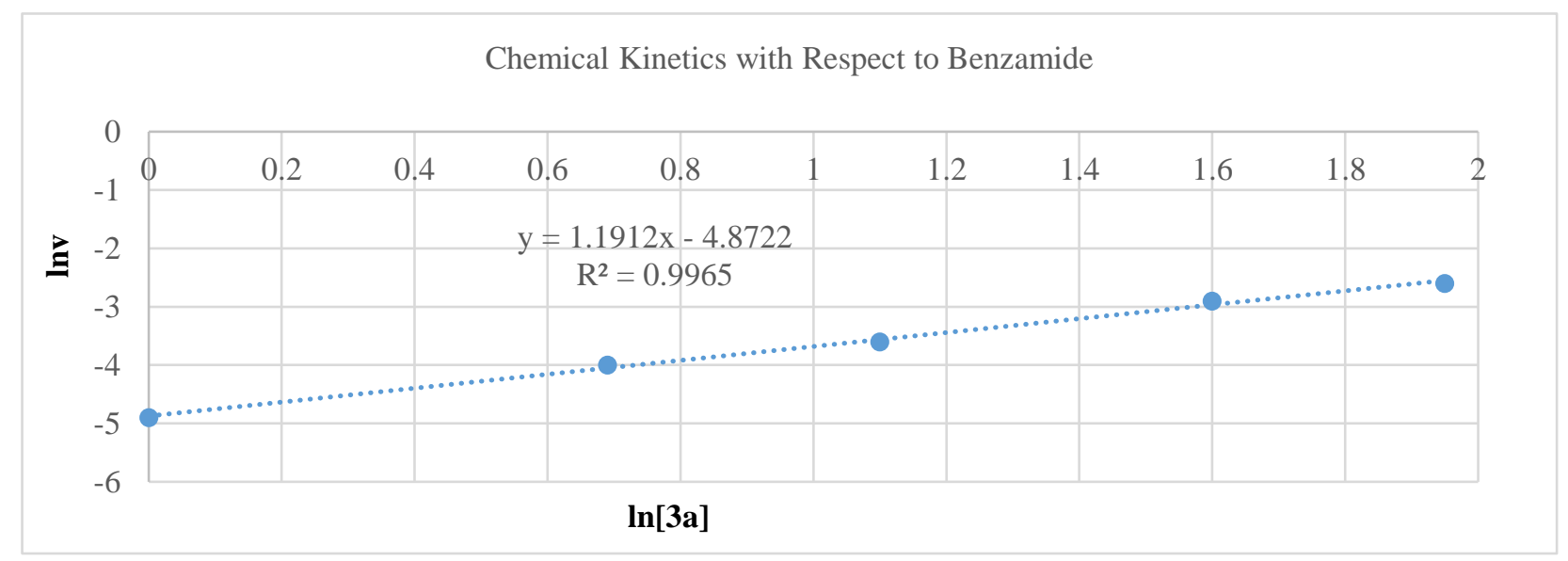

Figure S8. Graphical plot of $\ln (v)$ vs $\ln [\mathbf{3 a}]$

$\ln (v)=n \ln [3 \mathbf{a}]+C$ where $n$ is the order of the reaction and $\mathrm{C}$ is a constant

Observed $\mathrm{n}=1.1912 \approx 1.0$

Therefore, the observed rate of reaction is 1 with respect to the benzamide

\subsection{Kinetics with Respect to Base for $\mathrm{C}-\mathrm{N}$ Coupling Reaction}

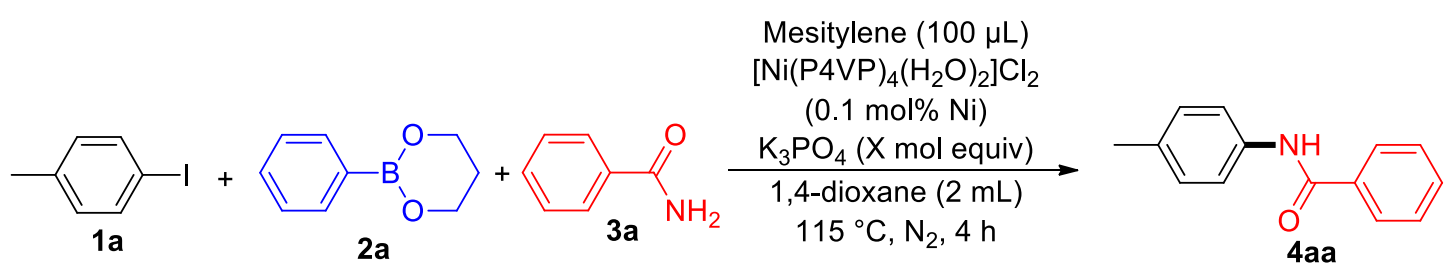

A mixture of $\left[\mathrm{Ni}(\mathrm{P} 4 \mathrm{VP})_{4}\left(\mathrm{H}_{2} \mathrm{O}\right)_{2}\right] \mathrm{Cl}_{2}(0.1 \mathrm{~mol} \% \mathrm{Ni})$, aryl iodide, $1 \mathbf{a}(1 \mathrm{~mol}$ equiv, $0.5 \mathrm{mmol})$, benzamide, 3a (3 mol equiv, $1.5 \mathrm{mmol}$ ), arylboronic ester, $2 \mathrm{a}(1.5 \mathrm{~mol}$ equiv, $0.75 \mathrm{mmol})$, and $\mathrm{K}_{3} \mathrm{PO}_{4}(\mathrm{X}$ mol equiv) were added to a reaction tube. The reaction tube was degassed under vacuum and refile with $\mathrm{N}_{2}$ under standard Schlack techniques (3 times). Mesitylene $(100 \mu \mathrm{L})$ and 1,4-dioxane $(2 \mathrm{~mL})$ was added and seal the reaction tube with teflon, followed by stirring at $115^{\circ} \mathrm{C}$ (chemiStation) for $4 \mathrm{~h}$. After $3 \mathrm{~h}$ the reaction tube was opened inside a glove box to take a small amount of reaction mixture and the reaction tube was resealed and further placed over the chemiStation for an additional hour. 
The collected reaction mixture was filtered and the filtrate was analyzed by GC. After $4 \mathrm{~h}$ the reaction mixture was opened and filtered. The filtrate was analyzed by GC for $4 \mathrm{~h}$ data.

\begin{tabular}{|c|c|c|c|c|c|}
\hline \multirow[t]{2}{*}{$\mathrm{K}_{3} \mathrm{PO}_{4}$} & \multicolumn{2}{|c|}{$[\mathbf{4 a a}] \times 10^{6}$} & \multirow[t]{2}{*}{$\mathrm{v}=$ initial rate $\times 10^{6}$} & \multirow[t]{2}{*}{$\operatorname{lnv}$} & \multirow[t]{2}{*}{$\ln \left[\mathbf{K}_{3} \mathbf{P} \mathbf{O}_{4}\right]$} \\
\hline & $3 \mathrm{~h}$ & $4 \mathrm{~h}$ & & & \\
\hline 1 equiv & 0.0144 & 0.0327 & 0.0183 & -4.0 & 0 \\
\hline 2 equiv & 0.1889 & 0.2449 & 0.0560 & -2.9 & 0.69 \\
\hline 3 equiv & 0.4909 & 0.6406 & 0.1496 & -1.9 & 1.1 \\
\hline 4 equiv & 0.5459 & 0.9135 & 0.3676 & -1.0 & 1.4 \\
\hline 5 equiv & 0.5561 & 1.0054 & 0.4493 & -0.8 & 1.6 \\
\hline
\end{tabular}

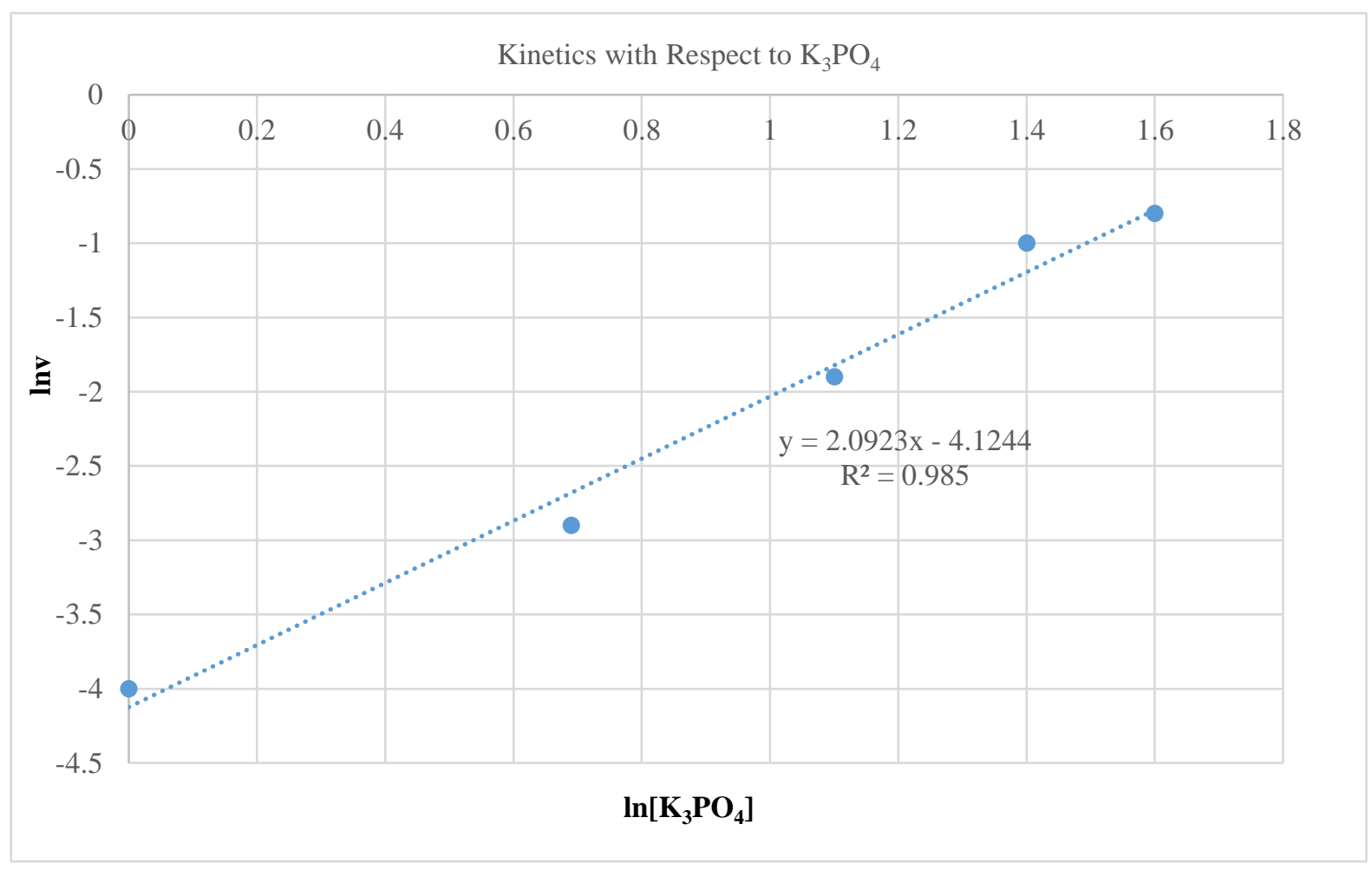

Figure S9. Graphical plot of $\operatorname{lnv}$ vs $\ln \left[\mathrm{K}_{3} \mathrm{PO}_{4}\right]$

$\operatorname{lnv}=\mathrm{n} \ln [\mathbf{b a s e}]+\mathrm{C}$ where $\mathrm{n}$ is the order of the reaction and $\mathrm{C}$ is a constant

Observed $\mathrm{n}=2.0923 \approx 2.0$

Therefore, the observed rate of reaction is 2 with respect to the $\mathrm{K}_{3} \mathrm{PO}_{4}$

\section{Chemical Kinetics for Suzuki-Miyaura-type Reaction}

\subsection{Kinetics with Respect to Catalyst for C-N Coupling Reaction}




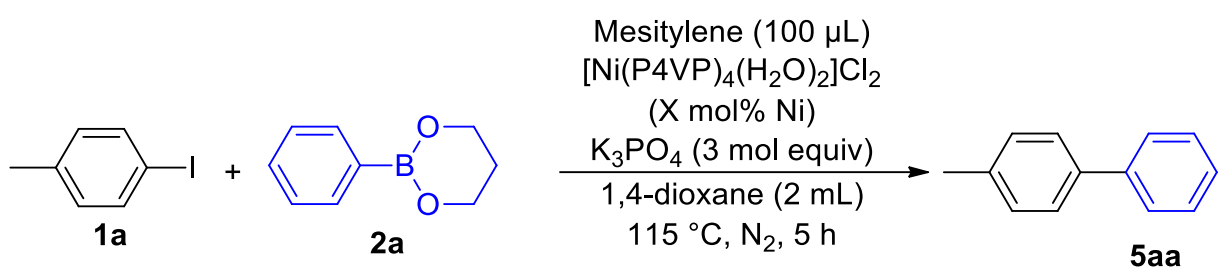

A mixture of $\left[\mathrm{Ni}(\mathrm{P} 4 \mathrm{VP})_{4}\left(\mathrm{H}_{2} \mathrm{O}\right)_{2}\right] \mathrm{Cl}_{2}(\mathrm{X}$ mol \% Ni), aryl iodide, $1 \mathbf{a}(1 \mathrm{~mol}$ equiv, $0.5 \mathrm{mmol})$, arylboronic ester, 2a (5 mol equiv, $2.5 \mathrm{mmol})$, and $\mathrm{K}_{3} \mathrm{PO}_{4}(3 \mathrm{~mol}$ equiv, $1.5 \mathrm{mmol}, 318 \mathrm{mg}$ ) were added to a reaction tube. The reaction tube was degassed under vacuum and refile with $\mathrm{N}_{2}$ under standard Schlack techniques (3 times). Mesitylene (100 $\left.\mu \mathrm{L}\right)$ and 1,4-dioxane $(2 \mathrm{~mL})$ was added and seal the reaction tube with teflon, followed by stirring at $115{ }^{\circ} \mathrm{C}$ (chemiStation) for $5 \mathrm{~h}$. After $4 \mathrm{~h}$ the reaction tube was opened inside a glove box to take a small amount of reaction mixture and the reaction tube was resealed and further placed over the chemiStation for an additional hour. The collected reaction mixture was filtered and the filtrate was analyzed by GC. After $5 \mathrm{~h}$ the reaction mixture opened and filtered. The filtrate was analyzed by GC.

\begin{tabular}{|c|c|c|c|c|c|c|}
\hline \multirow[t]{2}{*}{ [Cat $]$} & \multicolumn{2}{|c|}{$[\mathbf{1 a}] \times 10^{6}$} & \multirow{2}{*}{$\begin{array}{c}\mathrm{v}=\text { initial rate } \\
\times 10^{6}\end{array}$} & \multirow[t]{2}{*}{$\operatorname{lnv}$} & \multirow[t]{2}{*}[\text{cat}]{$^{*}=10 \times[$ cat $]$} & \multirow[t]{2}{*}{$\ln [\text { cat }]^{*}$} \\
\hline & $4 \mathrm{~h}$ & $5 \mathrm{~h}$ & & & & \\
\hline $0.1 \mathrm{~mol} \%$ & 0.3501 & 0.3167 & 0.0334 & -3.4 & 1 & 0 \\
\hline $0.2 \mathrm{~mol} \%$ & 0.1934 & 0.1526 & 0.0408 & -3.2 & 2 & 0.69 \\
\hline $0.3 \mathrm{~mol} \%$ & 0.2195 & 0.1826 & 0.0369 & -3.3 & 3 & 1.1 \\
\hline $0.4 \mathrm{~mol} \%$ & 0.0417 & 0.0083 & 0.0334 & -3.4 & 4 & 1.4 \\
\hline $0.5 \mathrm{~mol} \%$ & 0.2149 & 0.1780 & 0.0369 & -3.3 & 5 & 1.6 \\
\hline
\end{tabular}

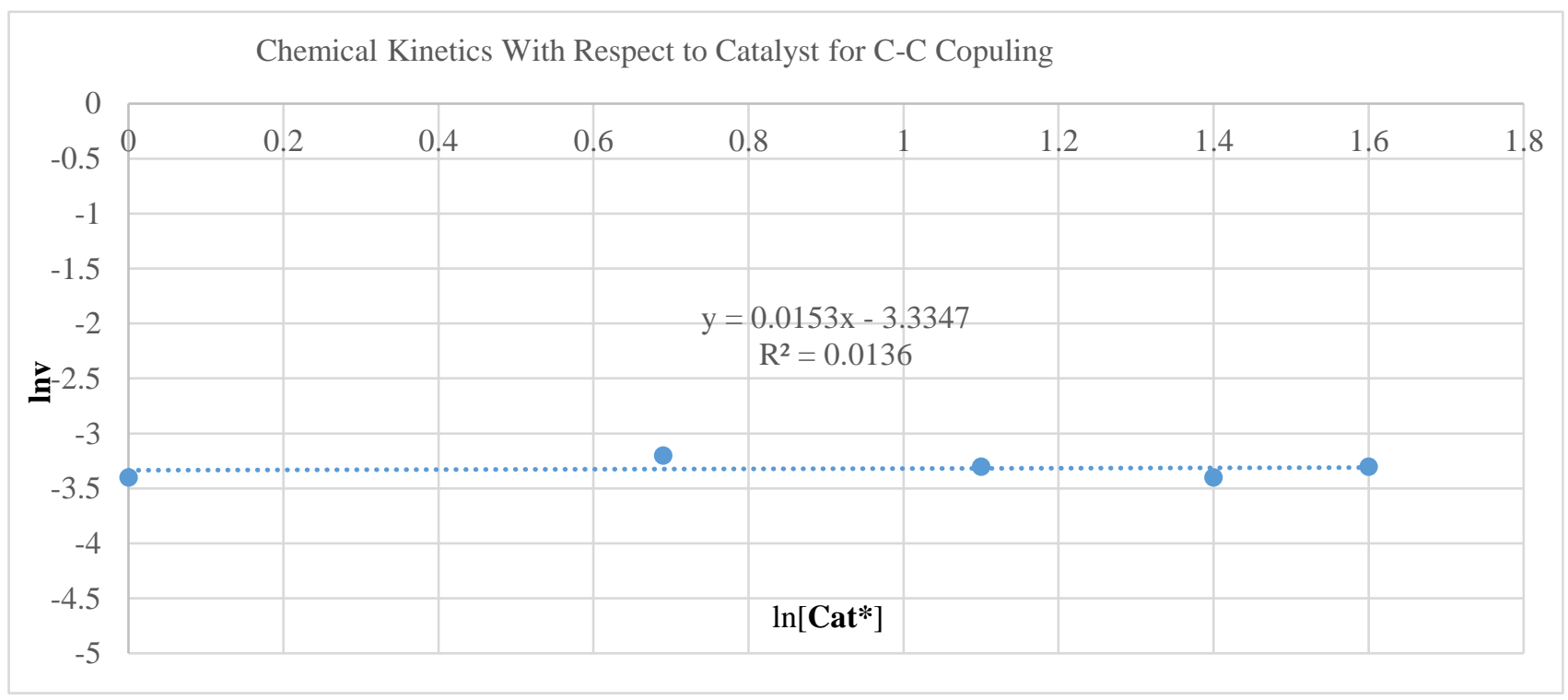

Figure S10. Graphical plot of $\operatorname{lnv}$ vs $\ln \left[\right.$ Cat*$\left.^{*}\right]$ 
$\operatorname{lnv}=n \ln \left[\mathbf{C a t}^{*}\right]+\mathrm{C}$ where $\mathrm{n}$ is the order of the reaction and $\mathrm{C}$ is a constant

$$
\text { Observed } \mathrm{n}=0.0253 \approx 0.0
$$

Therefore, the observed rate of reaction is 0 with respect to the catalyst

\subsection{Kinetics with Respect to Aryl Iodide for C-C Coupling}
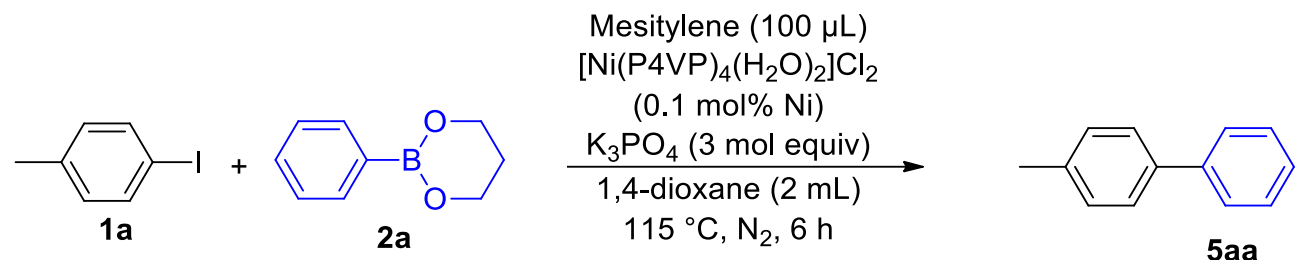

A mixture of $\left[\mathrm{Ni}(\mathrm{P} 4 \mathrm{VP})_{4}\left(\mathrm{H}_{2} \mathrm{O}\right)_{2}\right] \mathrm{Cl}_{2}(0.1 \mathrm{~mol} \% \mathrm{Ni})$, aryl iodide, 1a (X mol equiv), phenylboronic ester, $2 \mathbf{a}(5 \mathrm{~mol}$ equiv, $2.5 \mathrm{mmol}$ ), and $\mathrm{K}_{3} \mathrm{PO}_{4}(3 \mathrm{~mol}$ equiv, $1.5 \mathrm{mmol}, 318 \mathrm{mg})$ were added to a reaction tube. The reaction tube was degassed under vacuum and refile with $\mathrm{N}_{2}$ under standard Schlack techniques (3 times). Mesitylene (100 $\left.\mu \mathrm{L}\right)$ and 1,4dioxane $(2 \mathrm{~mL})$ was added and seal the reaction tube with teflon, followed by stirring at $115{ }^{\circ} \mathrm{C}$ (chemiStation) for 6 $\mathrm{h}$. After $5 \mathrm{~h}$ the reaction tube was opened inside a glove box to take a small amount of reaction mixture and the reaction tube was resealed and further placed over the chemiStation for an additional hour. The collected reaction mixture was filtered and the filtrate was analyzed by GC. After $6 \mathrm{~h}$ the reaction mixture opened and filtered. The filtrate was analyzed by GC.

\begin{tabular}{cccccc}
\hline 1a & \multicolumn{2}{c}{$[\mathbf{1 a}] \times 10^{6}$} & $\mathrm{v}=$ initial rate $\times 10^{6}$ & $\operatorname{lnv}$ & $\ln [\mathbf{1 a}]$ \\
& $5 \mathrm{~h}$ & $6 \mathrm{~h}$ & & & \\
\hline 1 equiv & 0.4158 & 0.2875 & 0.1283 & -2.1 & 0 \\
& & & & & \\
2 equiv & 0.8341 & 0.6728 & 0.1613 & -2.0 & 0.69 \\
& & & & & 1.1 \\
3 equiv & 1.2826 & 1.1653 & 0.1173 & -2.1 & 1.4 \\
4 equiv & 1.6430 & 1.5339 & 0.1091 & -2.2 & 1.6 \\
\hline 5 equiv & 2.2629 & 2.1445 & 0.1184 & -2.1 & \\
\hline
\end{tabular}




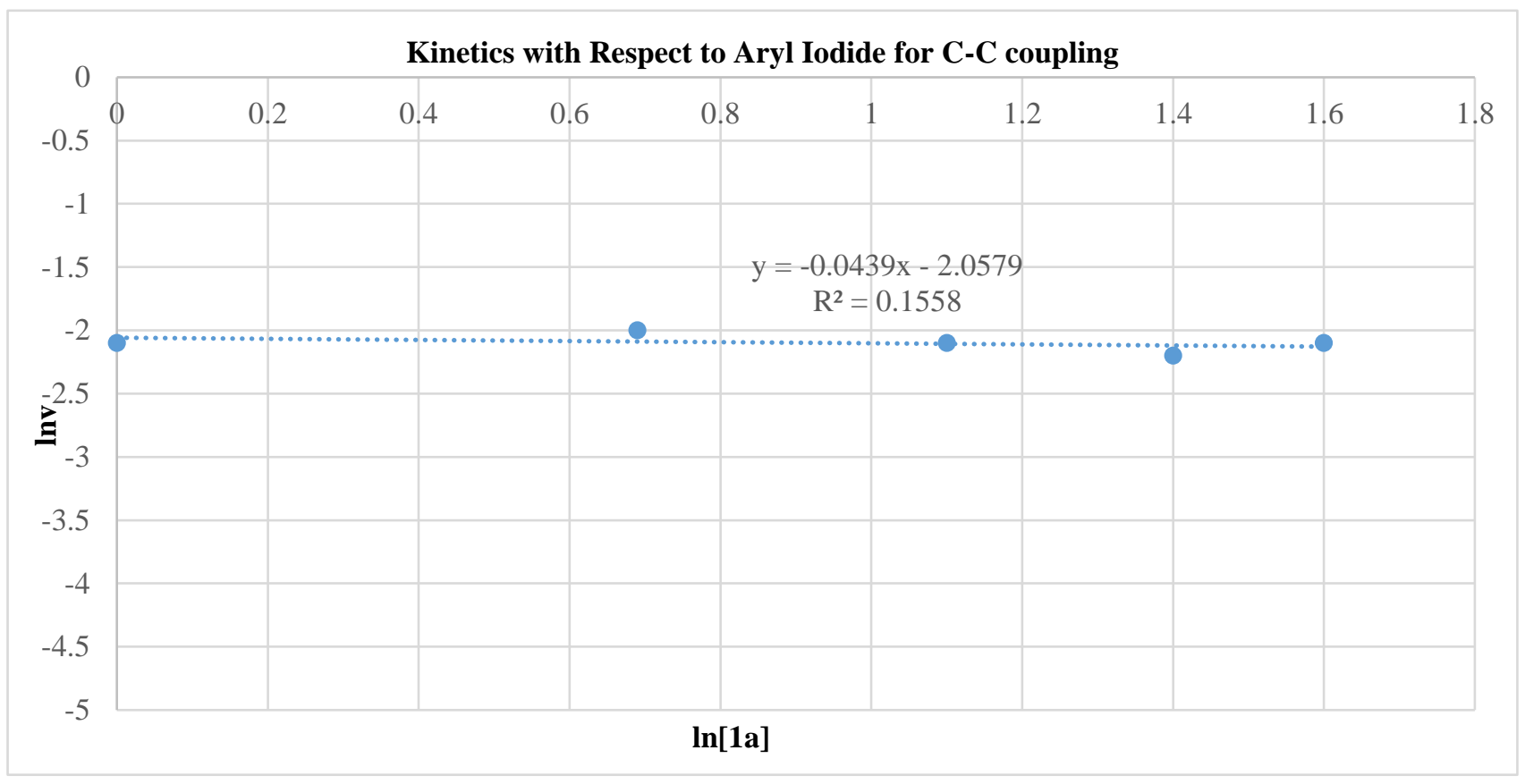

Figure S11. Graphical plot of $\operatorname{lnv}$ vs $\ln [\mathbf{1 a}]$

$\operatorname{lnv}=n \ln [\mathbf{1} \mathbf{a}]+\mathrm{C}$ where $\mathrm{n}$ is the order of the reaction and $\mathrm{C}$ is a constant

Observed $\mathrm{n}=0.0439 \approx 0.0$

Therefore, the observed rate of reaction is 0 with respect to the aryliodide

\subsection{Kinetics with Respect to Phenylboronic Acid for C-C Coupling}

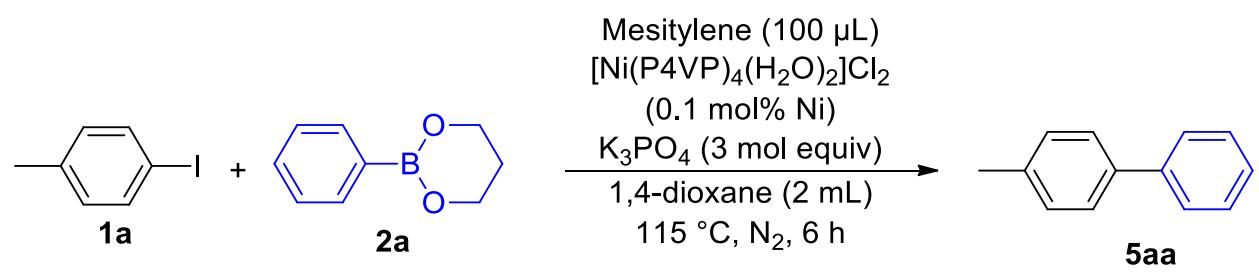

A mixture of $\left[\mathrm{Ni}(\mathrm{P} 4 \mathrm{VP})_{4}\left(\mathrm{H}_{2} \mathrm{O}\right)_{2}\right] \mathrm{Cl}_{2}(0.1 \mathrm{~mol} \% \mathrm{Ni})$, aryl iodide, $\mathbf{1 a}(1 \mathrm{~mol}$ equiv, $0.5 \mathrm{mmol})$, phenylboronic ester, 2a (X mol equiv), and $\mathrm{K}_{3} \mathrm{PO}_{4}(3 \mathrm{~mol}$ equiv, $1.5 \mathrm{mmol}, 318 \mathrm{mg}$ ) were added to a reaction tube. The reaction tube was degassed under vacuum and refile with $\mathrm{N}_{2}$ under standard Schlack techniques (3 times). Mesitylene (100 $\left.\mu \mathrm{L}\right)$ and 1,4dioxane $(2 \mathrm{~mL})$ was added and seal the reaction tube with teflon, followed by stirring at $115{ }^{\circ} \mathrm{C}$ (chemiStation) for 6 $\mathrm{h}$. After $5 \mathrm{~h}$ the reaction tube was opened inside a glove box to take a small amount of reaction mixture and the reaction tube was resealed and further placed over the chemiStation for an additional hour. The collected reaction mixture was filtered and the filtrate was analyzed by GC. After $6 \mathrm{~h}$ the reaction mixture opened and filtered. The filtrate was analyzed by GC.

\begin{tabular}{cccccc}
\hline $\mathbf{2 a}$ & \multicolumn{2}{c}{$[\mathbf{1 a}] \times 10^{6}$} & $\mathrm{v}=$ initial rate $\times 10^{6}$ & $\ln { }^{2}$ & $\ln [\mathbf{2 a}]$ \\
& $5 \mathrm{~h}$ & $6 \mathrm{~h}$ & & & \\
\hline 1 equiv & 0.3939 & 0.3857 & 0.0082 & -4.8 & 0
\end{tabular}




\begin{tabular}{lccccc}
2 equiv & 0.2305 & 0.2169 & 0.0136 & -4.3 & 0.69 \\
3 equiv & 0.2593 & 0.2369 & 0.0224 & -3.8 & 1.1 \\
4 equiv & 0.1425 & 0.1091 & 0.0334 & -3.4 & 1.4 \\
5 equiv & 0.3332 & 0.2782 & 0.0550 & -2.9 & 1.6 \\
\hline
\end{tabular}

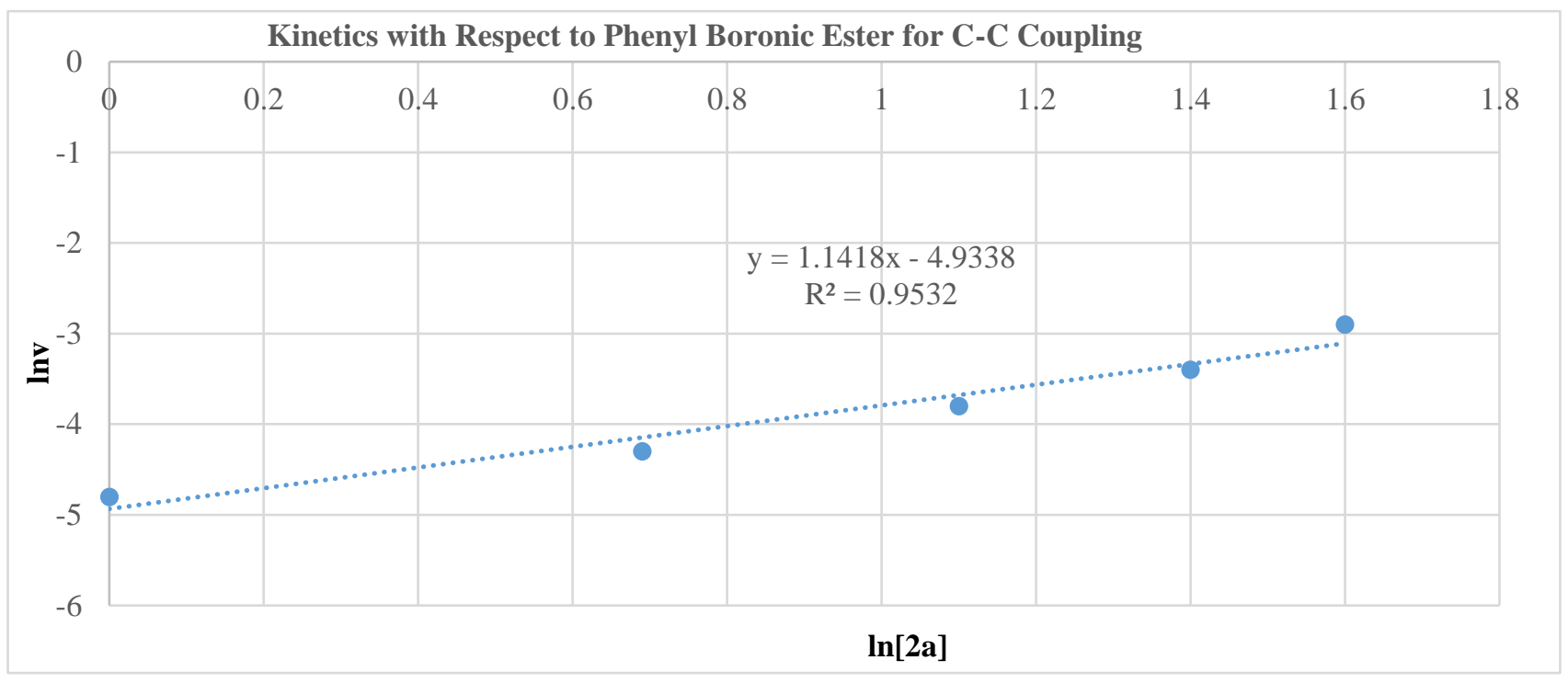

Figure S12. Graphical plot of $\operatorname{lnv}$ vs $\ln [\mathbf{2 a}]$

$\operatorname{lnv}=n \ln [\mathbf{2} \mathbf{a}]+\mathrm{C}$ where $\mathrm{n}$ is the order of the reaction and $\mathrm{C}$ is a constant

$$
\text { Observed } \mathrm{n}=1.1418 \approx 1.0
$$

Therefore, the observed rate of reaction is 1 with respect to the phenylboronic acid

\subsection{Kinetics with Respect to $\mathrm{K}_{3} \mathrm{PO}_{4}$ for C-C Coupling}

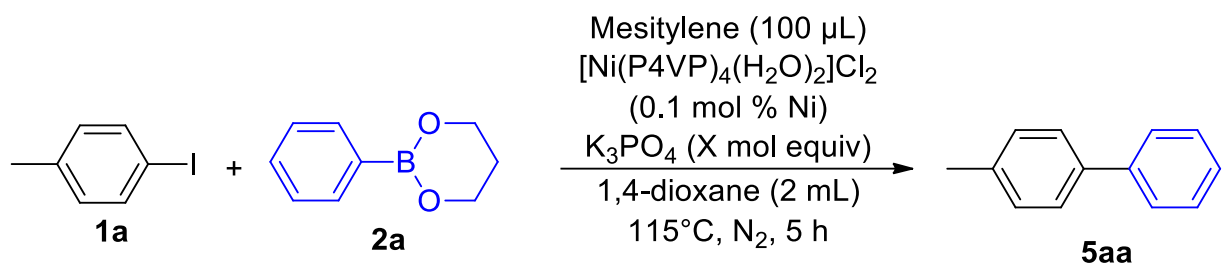

A mixture of $\left[\mathrm{Ni}(\mathrm{P} 4 \mathrm{VP})_{4}\left(\mathrm{H}_{2} \mathrm{O}\right)_{2}\right] \mathrm{Cl}_{2}(0.1 \mathrm{~mol} \% \mathrm{Ni})$, aryl iodide, $\mathbf{1 a}(1 \mathrm{~mol}$ equiv, $0.5 \mathrm{mmol})$, phenylboronic ester, 2a ( 5 mol equiv, $2.5 \mathrm{mmol}$ ), and $\mathrm{K}_{3} \mathrm{PO}_{4}$ (X mol equiv) were added to a reaction tube. The reaction tube was degassed under vacuum and refile with $\mathrm{N}_{2}$ under standard Schlack techniques (3 times). Mesitylene (100 $\left.\mu \mathrm{L}\right)$ and 1,4-dioxane $\left(2 \mathrm{~mL}\right.$ ) was added and seal the reaction tube with teflon, followed by stirring at $115^{\circ} \mathrm{C}$ (chemiStation) for $5 \mathrm{~h}$. After $4 \mathrm{~h}$ the reaction tube was opened inside a glove box to take a small amount of reaction mixture and the reaction tube was resealed and further placed over the chemiStation for an additional hour. The collected reaction mixture was filtered and the filtrate was analyzed by GC. After $5 \mathrm{~h}$ the reaction mixture opened and filtered. The filtrate was analyzed by GC. 


\begin{tabular}{|c|c|c|c|c|c|}
\hline \multirow[t]{2}{*}{$\mathrm{K}_{3} \mathrm{PO}_{4}$} & \multicolumn{2}{|c|}{$[\mathbf{1 a}] \times 10^{6}$} & \multirow[t]{2}{*}{$\mathrm{v}=$ initial rate $\times 10^{6}$} & \multirow[t]{2}{*}{$\operatorname{lnv}$} & \multirow[t]{2}{*}{$\ln \left[\mathbf{K}_{3} \mathbf{P O}_{4}\right]$} \\
\hline & $4 \mathrm{~h}$ & $5 \mathrm{~h}$ & & & \\
\hline 1 equiv & 0.3481 & 0.3286 & 0.0195 & -3.9 & 0 \\
\hline 2 equiv & 0.2360 & 0.2026 & 0.0334 & -3.4 & 0.69 \\
\hline 3 equiv & 0.3440 & 0.2953 & 0.0487 & -3.0 & 1.1 \\
\hline 4 equiv & 0.4768 & 0.3947 & 0.0821 & -2.5 & 1.4 \\
\hline 5 equiv & 0.1602 & 0.0432 & 0.1170 & -2.1 & 1.6 \\
\hline
\end{tabular}

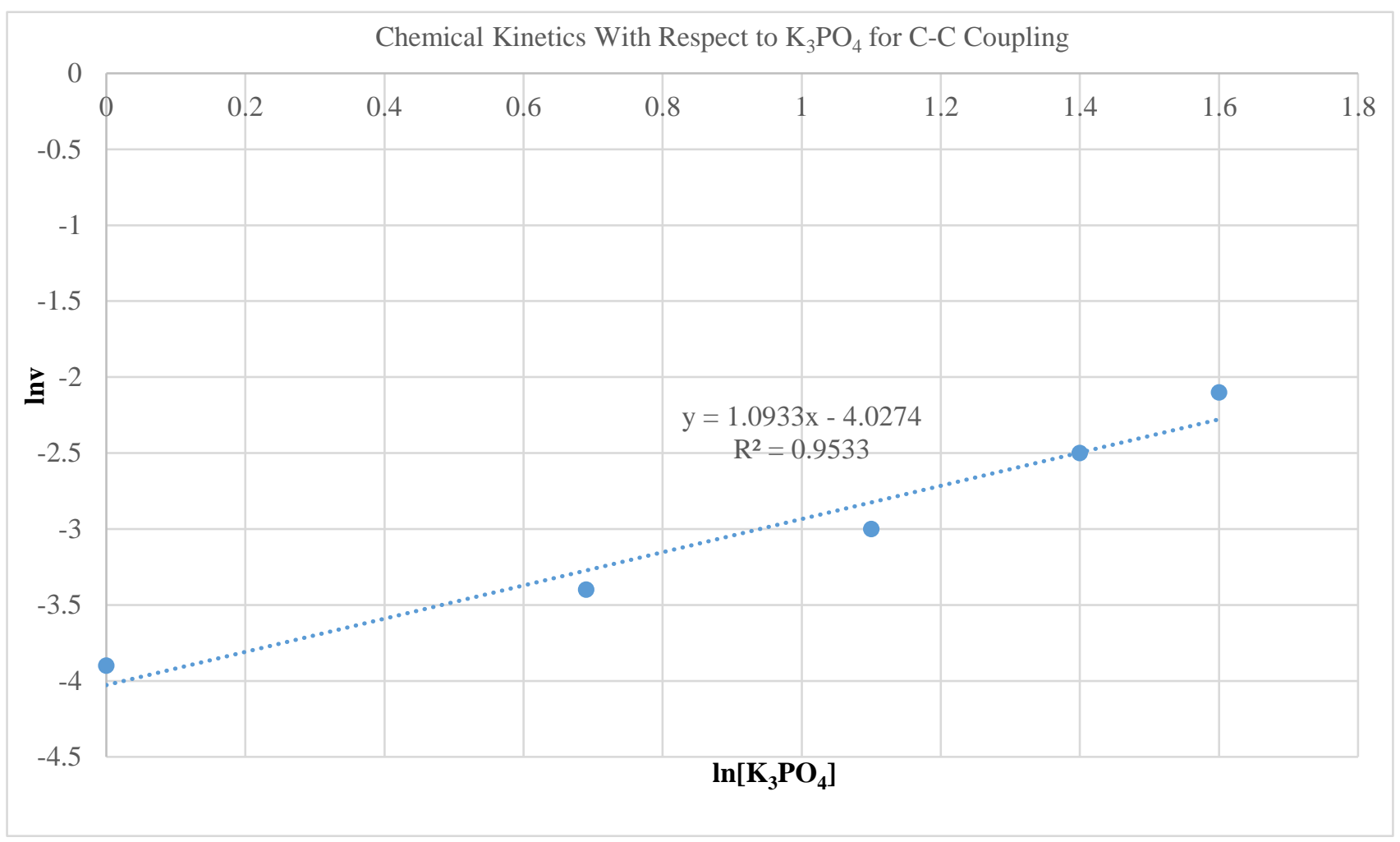

Figure S13. Graphical plot of $\operatorname{lnv}$ vs $\ln \left[\mathrm{K}_{3} \mathbf{P O}_{4}\right]$

$\operatorname{lnv}=n \ln \left[\mathbf{K}_{3} \mathbf{P O}_{4}\right]+\mathrm{C}$ where $\mathrm{n}$ is the order of the reaction and $\mathrm{C}$ is a constant

Observed $\mathrm{n}=1.0933 \approx 1.0$

Therefore, the observed rate of reaction is 1 with respect to the $\mathrm{K}_{3} \mathrm{PO}_{4}$

\section{Effect of Benzamide Addition in Suzuki-Miyaura Coupling Reaction}

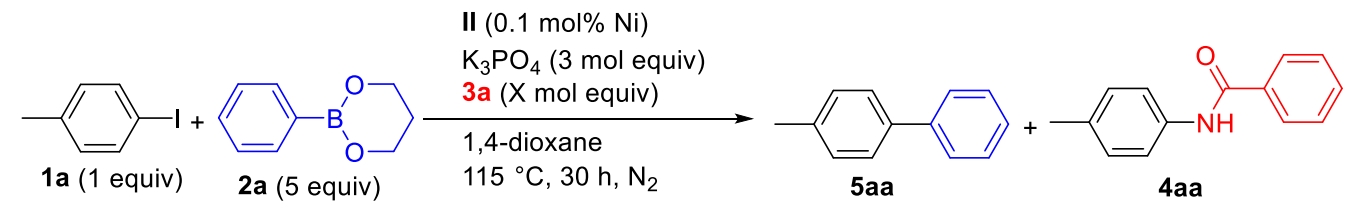


A mixture of $\left[\mathrm{Ni}(\mathrm{P} 4 \mathrm{VP})_{4}\left(\mathrm{H}_{2} \mathrm{O}\right)_{2}\right] \mathrm{Cl}_{2}(1000 \mathrm{~mol} \mathrm{ppm}, 0.42 \mathrm{mg})$, aryl iodide, 1 (1 mol equiv, $\left.0.5 \mathrm{mmol}\right)$, arylboronic ester, 2 (5 mol equiv, $2.5 \mathrm{mmol}$ ), Benzamide 3a (X mol equiv, 10 reactions) and $\mathrm{K}_{3} \mathrm{PO}_{4}(3 \mathrm{~mol}$ equiv, $1.5 \mathrm{mmol}, 318 \mathrm{mg}$ ) were added to a reaction tube. The reaction tubes were degassed under vacuum and refile with $\mathrm{N}_{2}$ under standard Schlack techniques ( 3 times). 1,4-dioxane $(2 \mathrm{~mL})$ was added and seal the reaction tube with teflon, followed by stirring at $115{ }^{\circ} \mathrm{C}$ (chemiStation) under nitrogen for $30 \mathrm{~h}$. After that, the reaction mixture was diluted with EtOAc and checked with GC with mesitylene as an internal standard.

\begin{tabular}{cccc}
\hline entry & amount of benzamide added (X equiv) & yield & yield \\
& & 5aa $(\%)$ & 4aa (\%) \\
\hline 1 & 0 & 91 & 0 \\
2 & 0.1 & 90 & 18 \\
3 & 0.2 & 79 & 30 \\
4 & 0.4 & 68 & 57 \\
5 & 0.6 & 43 & 68 \\
6 & 0.8 & 29 & 75 \\
7 & 1 & 18 & 82 \\
9 & 3 & 15 & 91 \\
10 & 5 & 12 & \\
\hline
\end{tabular}




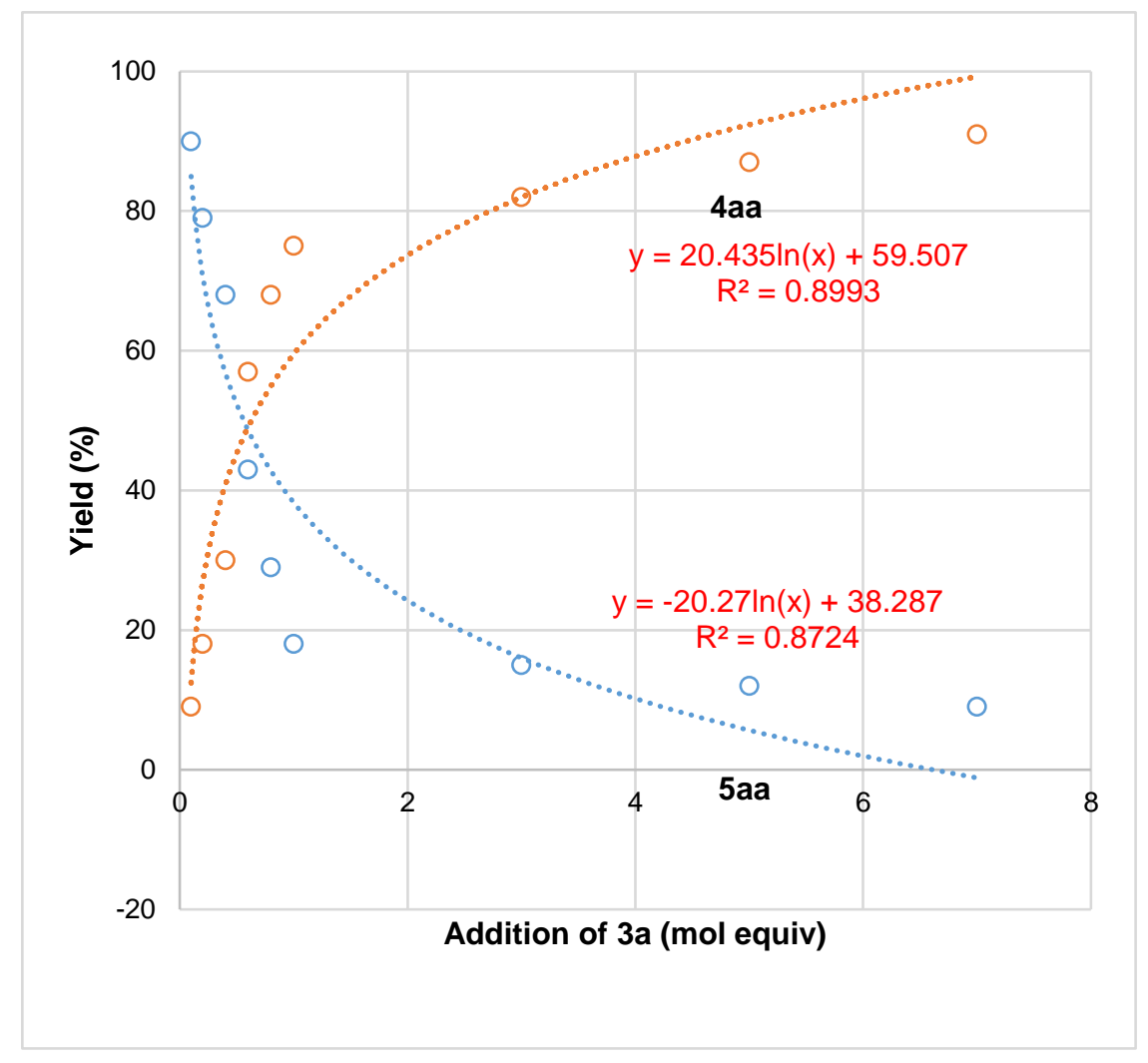

Figure S14. Effect of Benzamide Addition

As the amount of benzamide increases the yield of Suzuki-Miyaura coupling decrease whereas the yield of amidation reaction increases. So by controlling the amount of amide the product switch can also be controlled.

\section{Effect of Phenylboronic Acid Addition in Amidation Reaction}

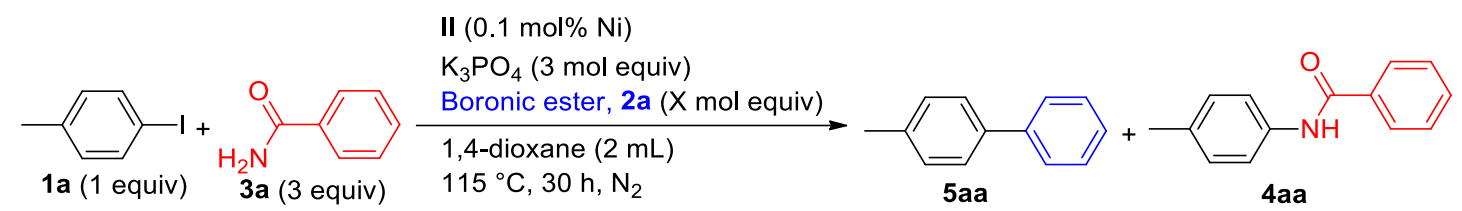

A mixture of $\left[\mathrm{Ni}(\mathrm{P} 4 \mathrm{VP})_{4}\left(\mathrm{H}_{2} \mathrm{O}\right)_{2}\right] \mathrm{Cl}_{2}(1000 \mathrm{~mol} \mathrm{ppm}, 0.42 \mathrm{mg})$, aryl iodide, 1a (1 mol equiv, $\left.0.5 \mathrm{mmol}, 109 \mathrm{mg}\right)$, phenylboronic ester, $2 \mathbf{a}$ (X mol equiv, 10 reactions), amide substrate $3 \mathbf{a}$ ( 3 mol equiv, 1.5 mmol, $184 \mathrm{mg}$ ) and $\mathrm{K}_{3} \mathrm{PO}_{4}(3$ mol equiv, $1.5 \mathrm{mmol}, 318 \mathrm{mg}$ ) were added to a reaction tube. The reaction tube was degassed under vacuum and refile with $\mathrm{N}_{2}$ under standard Schlack techniques (3 times). 1,4-dioxane ( $2 \mathrm{~mL}$ ) was added and seal the reaction tube with teflon, followed by stirring at $115^{\circ} \mathrm{C}$ (chemiStation) under nitrogen for $30 \mathrm{~h}$. After the reaction, the reaction mixture was diluted by EtOAc and analyzed by GC using mesitylene as an internal standard.

\begin{tabular}{cccc}
\hline entry & amount of boronic ester added (X equiv) & yield & yield \\
& 4aa (\%) & 5aa $(\%)$ \\
\hline 1 & 0 & 0 & 0
\end{tabular}




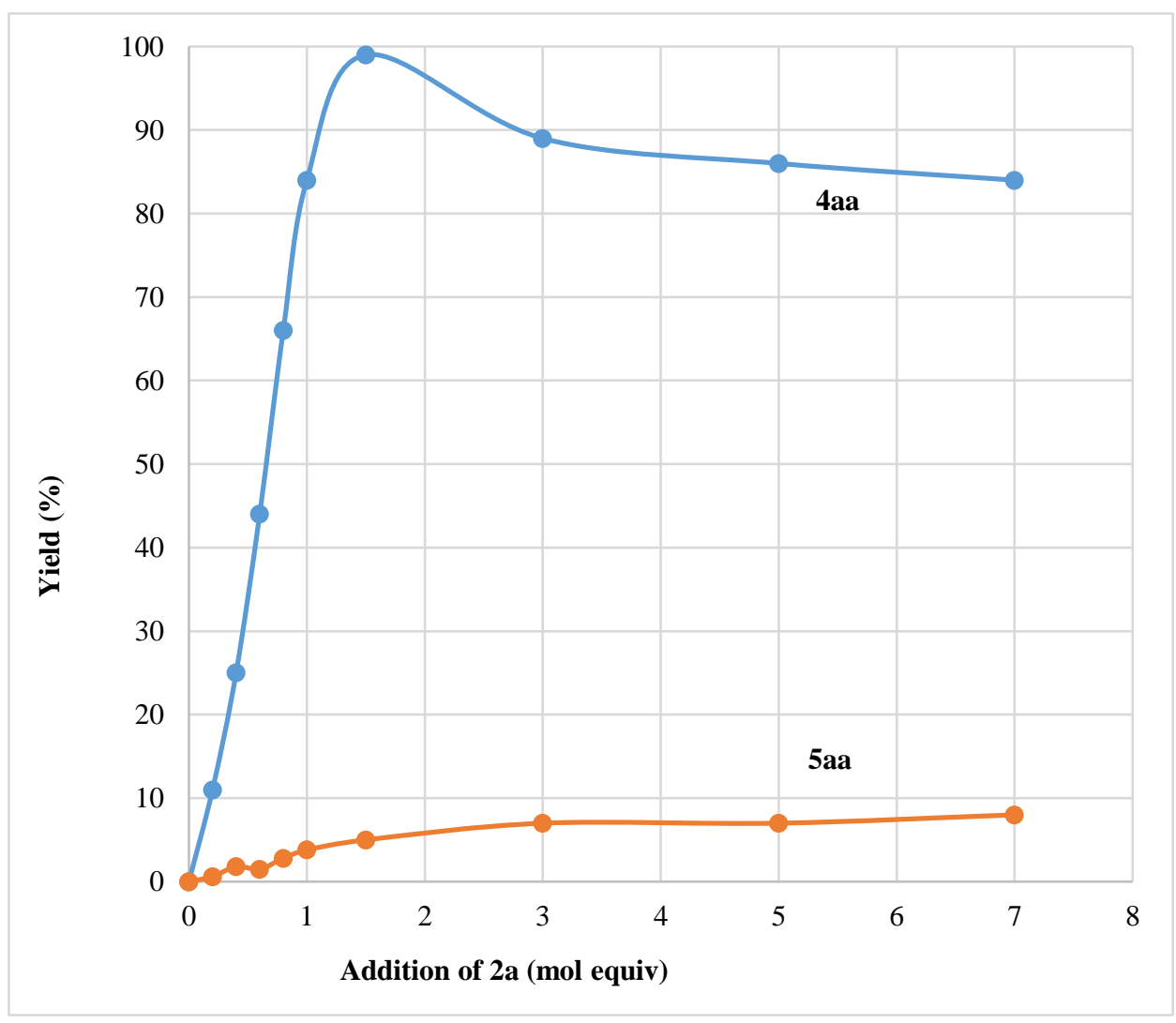

Figure S15. Effect of phenylboronic ester addition

As the amount of phenylboronic ester increases the yield of $\mathrm{C}-\mathrm{N}$ coupling as well as $\mathrm{C}-\mathrm{C}$ coupling increases until it reaches the optimum conditions for $\mathrm{C}-\mathrm{N}$ coupling reactions. After that, there was a decreasing trend for amidation reaction whereas an increasing trend in $\mathrm{C}-\mathrm{C}$ bond forming reactions although the overall yield for the $\mathrm{C}-\mathrm{C}$ bond 
forming reaction is very less. The amidation reaction is very fast compared to the $\mathrm{C}-\mathrm{C}$ bond formation reaction under P4VP-Ni catalysis as we observed from reaction time course measurements.

\section{Reaction Time Course Measurements}

\subsection{Time Course for Amidation Reaction}

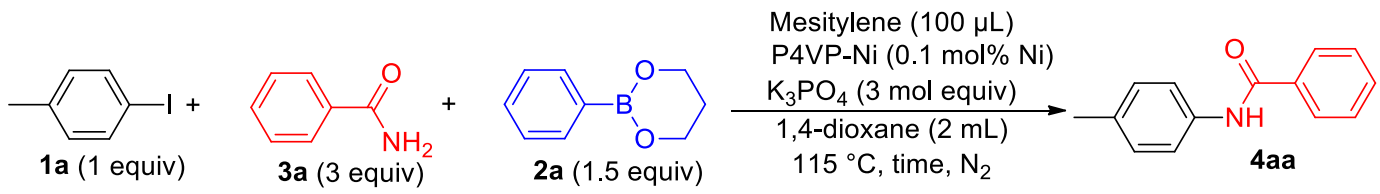

A set of 11 different reaction systems was prepared according to the general procedure for the C-N coupling reaction for $0.5 \mathrm{~h}$ to $30 \mathrm{~h}$. After the end of the desired time, the reaction mixture was diluted with EtOAc and filter. The filtrate was collected and analyzed by GC.

\begin{tabular}{ccc}
\hline entry & reaction time $(\mathrm{h})$ & 4aa \\
\hline 1 & 0.5 & 0 \\
2 & 1 & 3 \\
3 & 1.5 & 7 \\
4 & 2 & 14 \\
5 & 3 & 27 \\
6 & 4 & 36 \\
7 & 5 & 40 \\
8 & 6 & 56 \\
9 & 7 & 65 \\
10 & 24 & 96 \\
11 & 30 & 99 \\
\hline
\end{tabular}

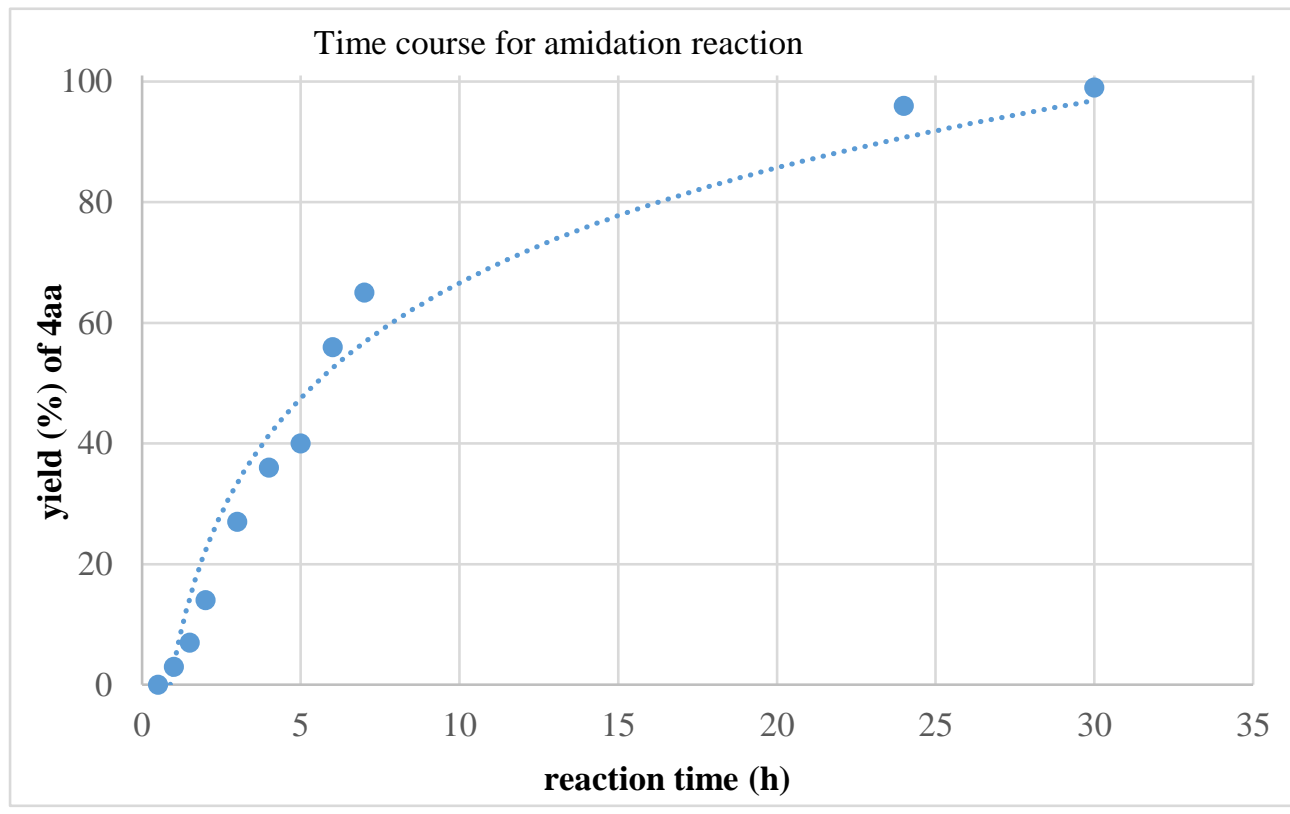

Figure S16. Reaction time course for amidation reaction 


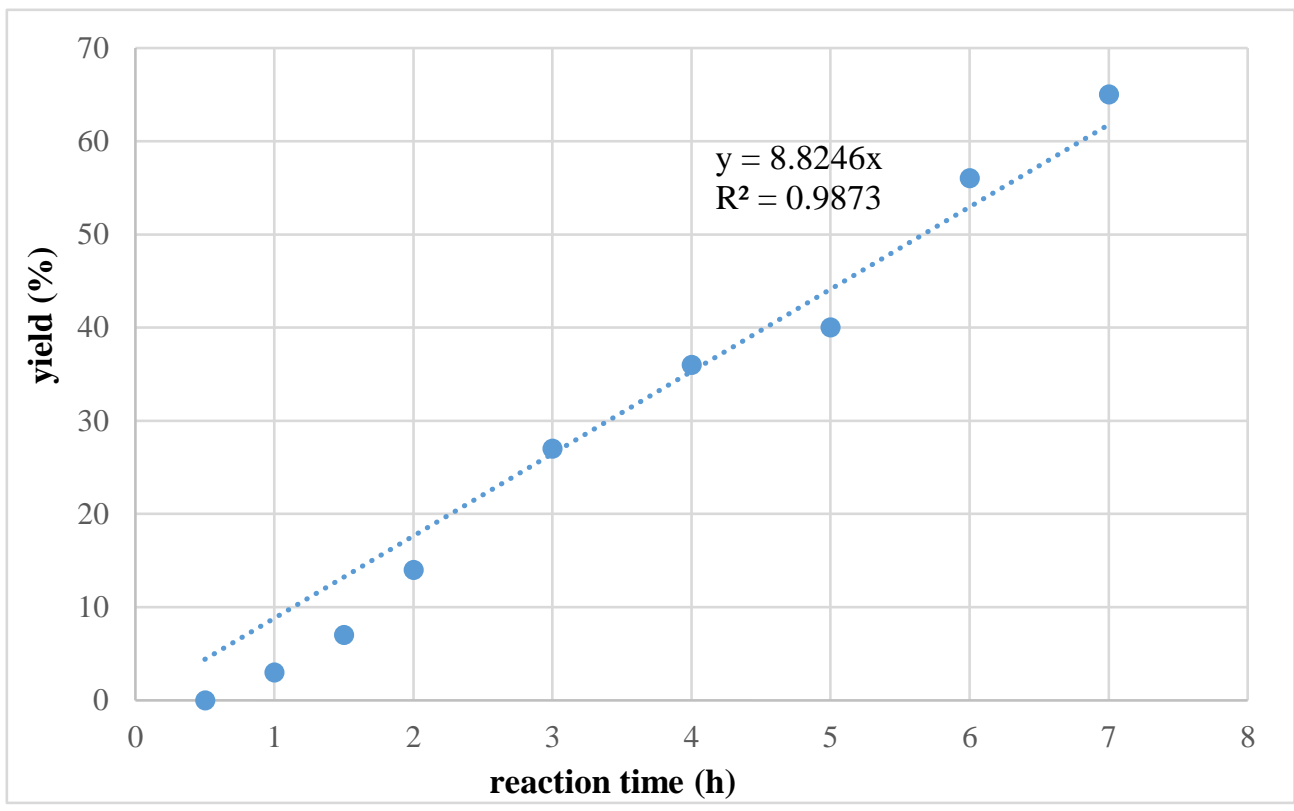

Figure S17. Initial time course for amidation reaction

\subsection{Time Course for C-C Coupling Reaction}

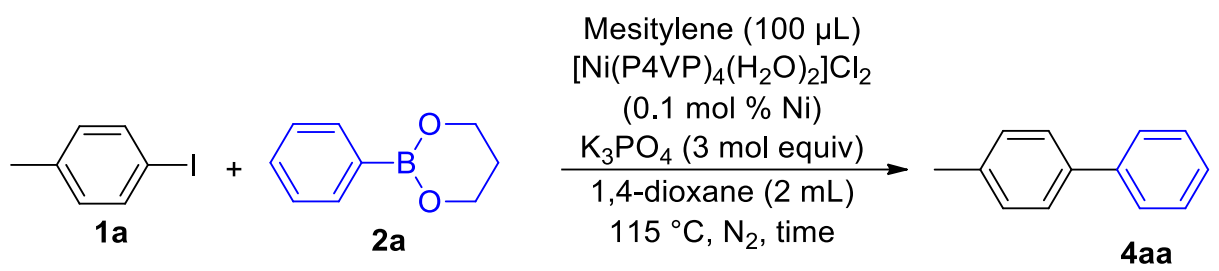

A set of 9 different reactions were prepared according to the general procedure for the $\mathrm{C}$-C coupling reaction for $1 \mathrm{~h}$ to $30 \mathrm{~h}$. After the end of the desired time, the reaction mixture was diluted with EtOAc and filter. The filtrate was collected and analyzed by GC.

\begin{tabular}{ccc}
\hline entry & reaction time $(\mathrm{h})$ & 5aa \\
\hline 1 & 1 & 1 \\
2 & 2 & 4 \\
3 & 3 & 8 \\
4 & 4 & 12 \\
5 & 5 & 15 \\
6 & 6 & 17 \\
7 & 7 & 22 \\
8 & 24 & 89 \\
9 & 30 & 91 \\
\hline
\end{tabular}




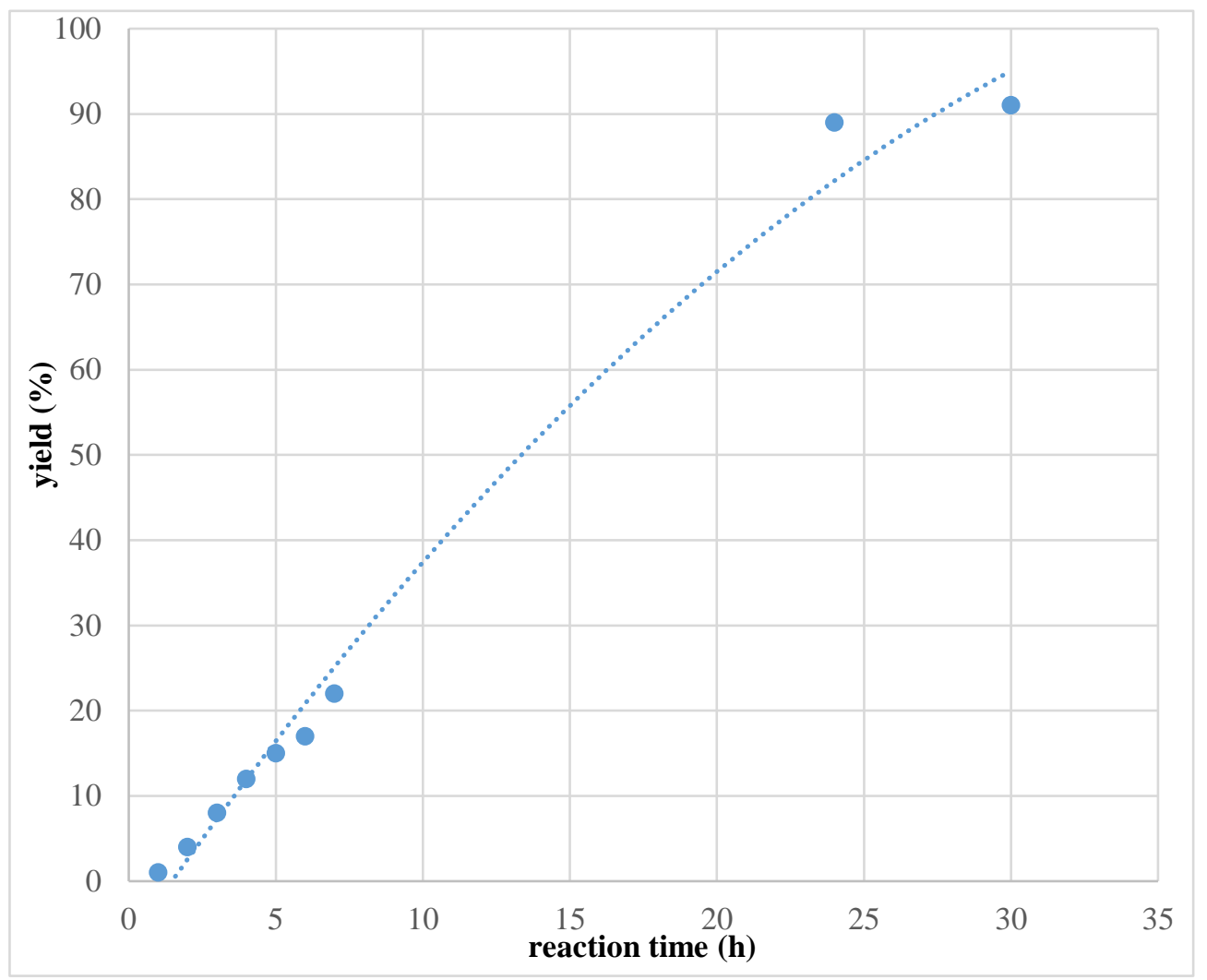

Figure S18. Time course for the C-C coupling reaction

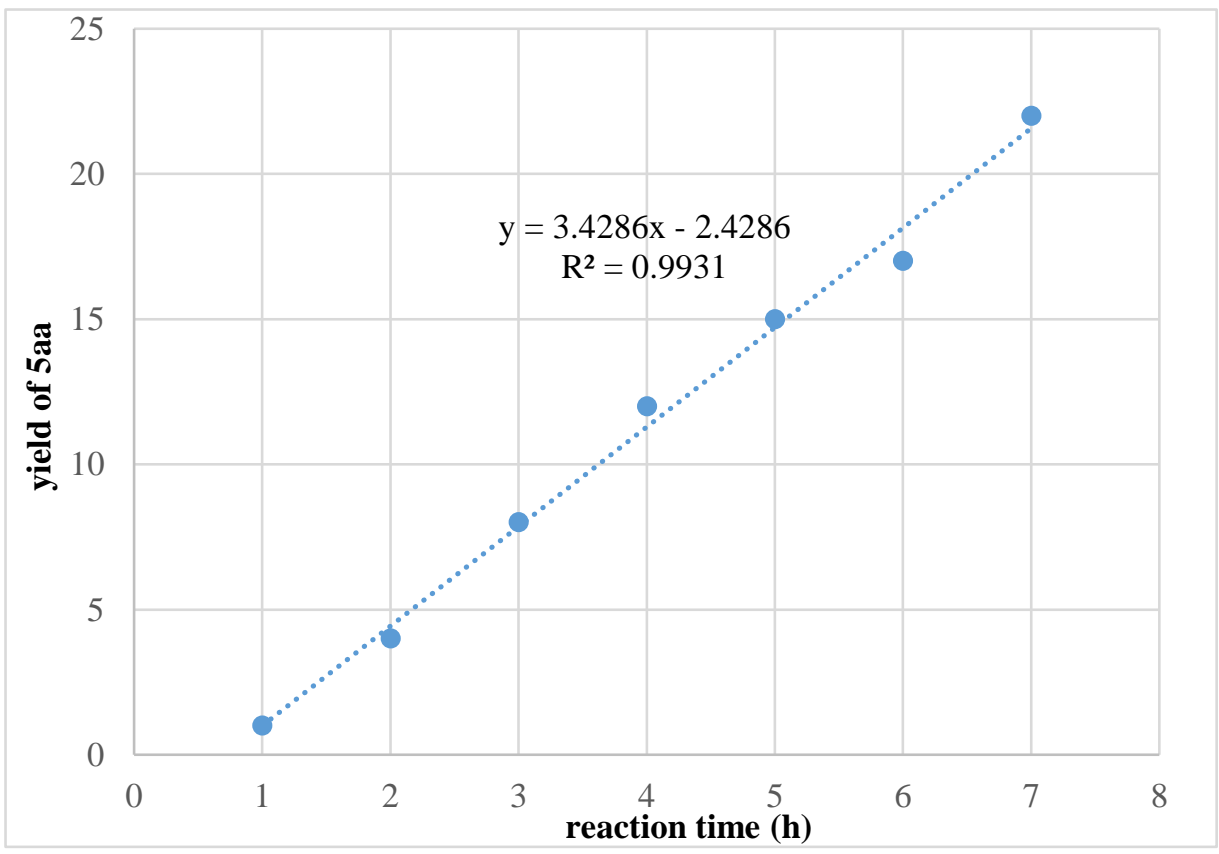

Figure S19. Initial time course for the C-C coupling reaction

\section{Catalyst Recovery and Application of Recovered Catalyst}

\subsection{Hot Filtration:}

Hot filtration test was performed to confirm the heterogeneous nature of the reaction. Initially we performed the reaction under the standard reaction conditions. After the 50\% (approximately) of starting material (1a) to the amidation product we performed the hot filtration (Omnipore Membrane Filters, $0.2 \mu \mathrm{m}$ ) and the filtrate was collected. The residue was 
washed with hot 1,4-dioxane. When we performed the reaction with the collected filtrate in presence or absence of additional 1 equivalent of $\mathrm{K}_{3} \mathrm{PO}_{4}$ the reaction didn't proceed further even after additional 24 hours.

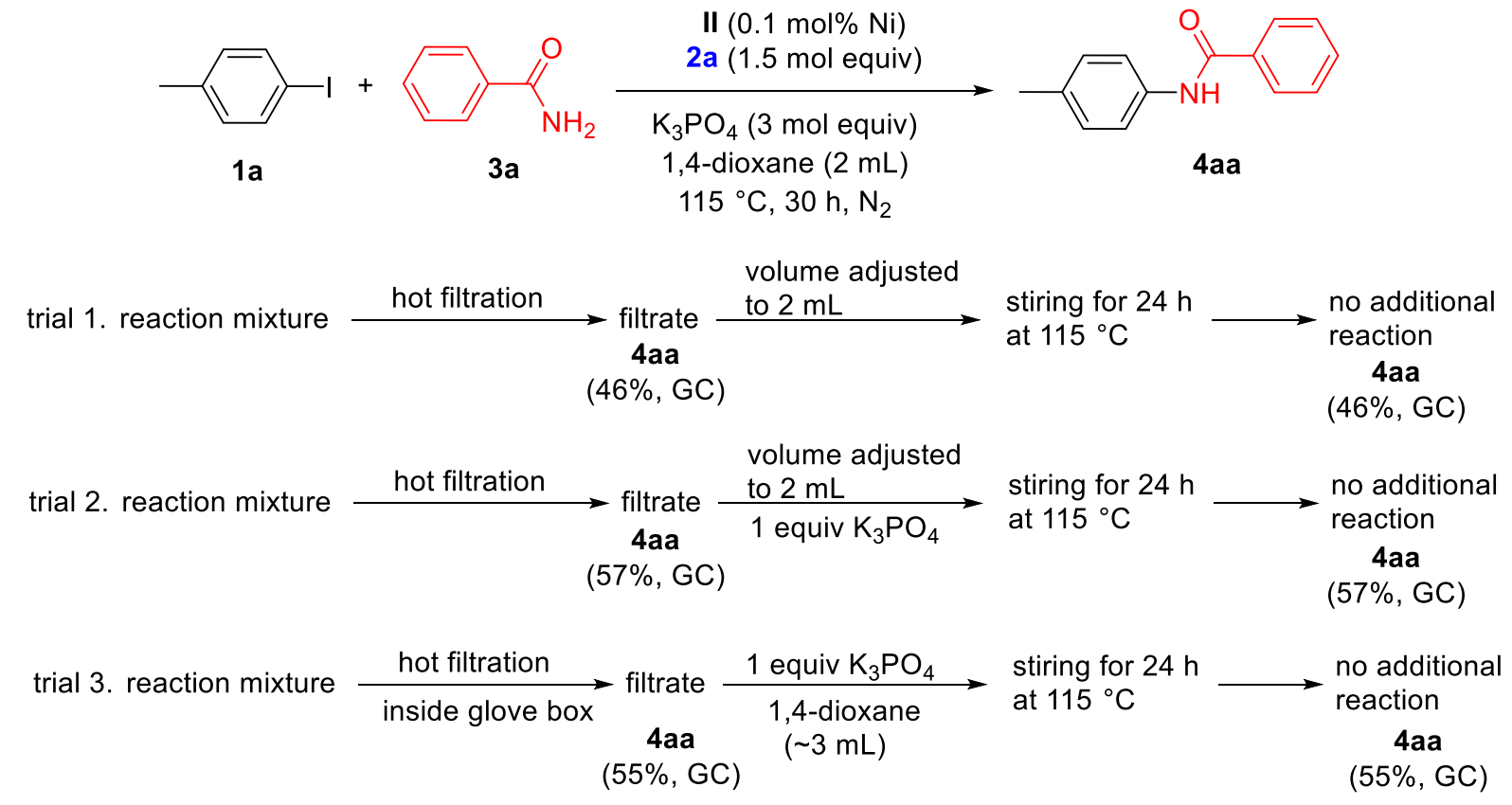

\subsection{Flow Chart for Catalyst Recovery}

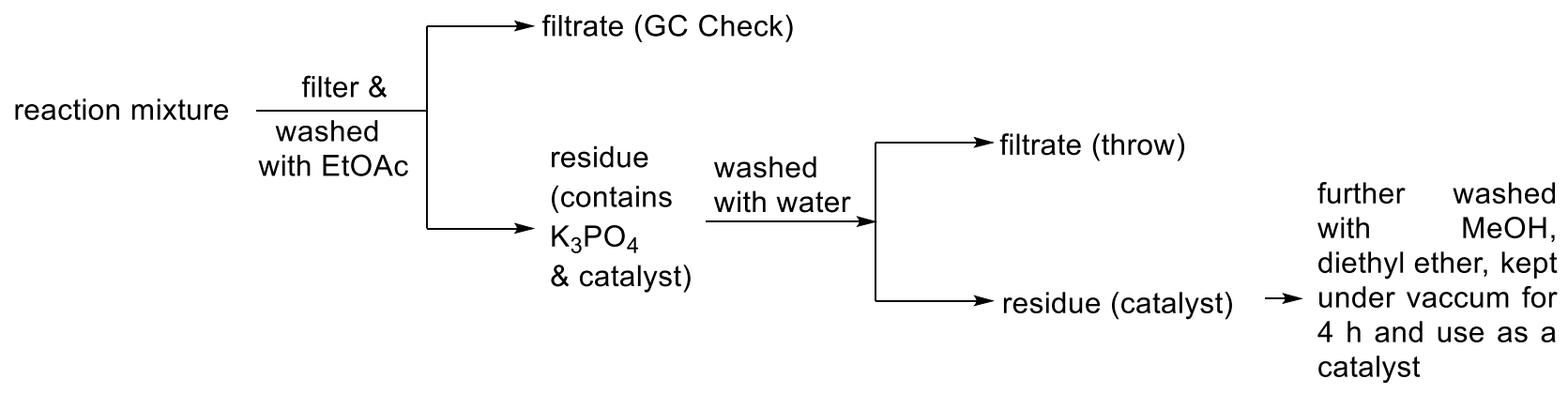

\subsection{Application of Recovered Catalyst in Amidation Reaction}

\begin{tabular}{cccc}
\hline entry & number of run & 4aa $(\%)$ & catalyst recovery yield (\%) \\
\hline 1 & $1^{\text {st }}$ & 99 & 91 \\
2 & $2^{\text {nd }}$ & 93 & 94 \\
3 & $3^{\text {rd }}$ & 96 & 85 \\
4 & $4^{\text {th }}$ & 89 & 89 \\
5 & $5^{\text {th }}$ & 88 & 82 \\
6 & $6^{\text {th }}$ & 85 & 79 \\
\hline
\end{tabular}

17.4. Catalyst Recovery from C-C Coupling Reaction and Apply the Recovered Catalyst for Amidation Reaction and Vice Versa 
P4VP-Ni

(0.1 mol\% Ni, 0. $46 \mathrm{mg})$

Eq. 1

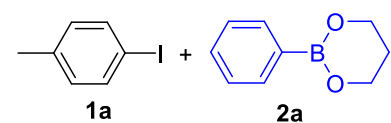

$\mathrm{K}_{3} \mathrm{PO}_{4}$ (3 mol equiv)

${ }^{\circ} \mathrm{C}, \mathrm{N}_{2}, 30 \mathrm{~h}, \mathrm{seal}$

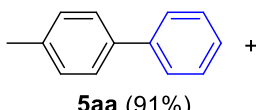

recovered cat1 $(93 \%, 0.43 \mathrm{mg})$

recovered cat 1

$(0.1 \mathrm{~mol} \% \mathrm{Ni}, 0.41 \mathrm{mg}$ )

Eq. $2 \longrightarrow+$

2a

$\mathrm{K}_{3} \mathrm{PO}_{4}$ (3 mol equiv)

1,4-dioxane ( $2 \mathrm{~mL}$ )

$115^{\circ} \mathrm{C}, \mathrm{N}_{2}, 30 \mathrm{~h}$, seal

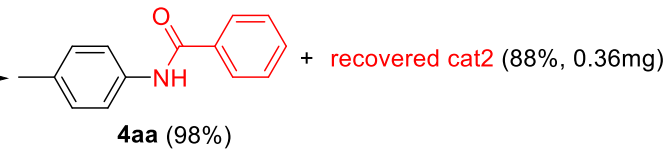

recovered cat2

$(0.1 \mathrm{~mol} \% \mathrm{Ni}, 0.33 \mathrm{mg})$

Eq. 3

1,4-dioxane (2 $\mathrm{mL})$

$115^{\circ} \mathrm{C}, \mathrm{N}_{2}, 30 \mathrm{~h}$, seal

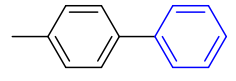

+ recovered cat3 $(80 \%, 0.27 \mathrm{mg})$

recovered cat3

$(0.1 \mathrm{~mol} \% \mathrm{Ni}, 0.27 \mathrm{mg})$

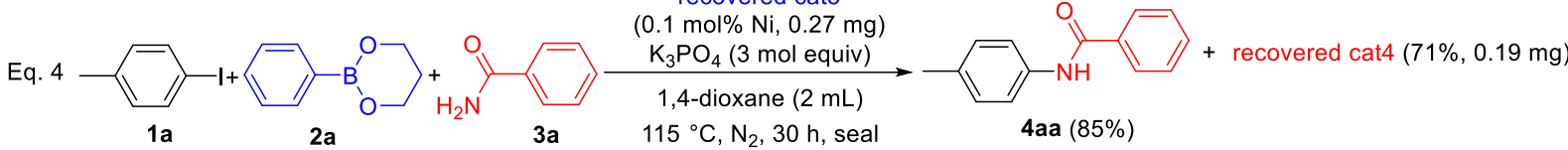

\section{Possible Pathway for Ni(I) Catalyst Generation: ${ }^{37}$}

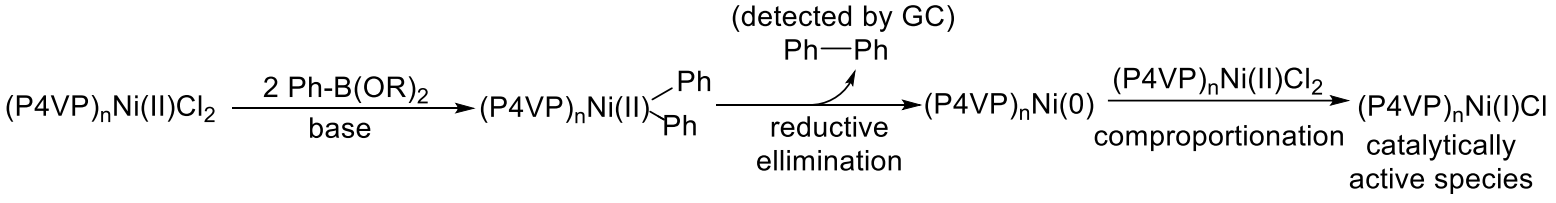

\section{ICP-MS Analysis:}

Inductively coupled plasma mass spectrometry (ICP-MS) measurement was performed using Perkin Elmer Nexion 300D. The sample was pretreated by microwave digestion technique with high-purity nitric acid (69\%, obtained from Kanto Chemical, Ultrapur-100 grade) as solvent (7 mL) using Perkin Elmer Titan MPS microwave sample preparation system. A nickel standard solution was obtained from Merck.

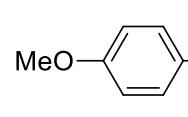

$1 \mathrm{e}$

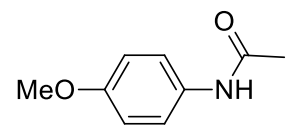

4eh

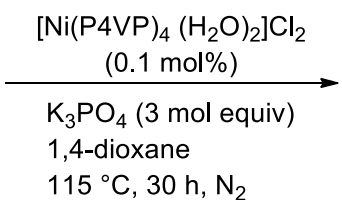

$2 a$

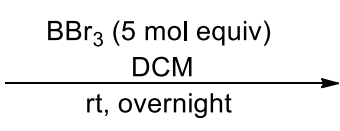

rt, overnight

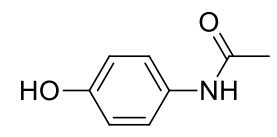

s1

paracetamol $98 \%$ yield

\section{ICP-MS Analysis of nickel in 4eh}


Solid product 4eh $(30 \mathrm{mg})$ was heated with nitric acid using a microwave heating system, and the resulted solution was diluted with water to $200 \mathrm{~mL}$ total. ICP-MS analysis of the solution showed that $1.7 \mathrm{mg} \mathrm{Kg}^{-1}$ (1.7 ppm) of nickel contaminated to the solid product (the limit of detection of ICP-MS: $0.6 \mathrm{mg} \mathrm{Kg}^{-1}$ ).

\section{ICP-MS Analysis of nickel in S1}

Solid product S1 (30 $\mathrm{mg})$ was heated with nitric acid using a microwave heating system, and the resulted solution was diluted with water to $200 \mathrm{~mL}$ total. ICP-MS analysis of the solution showed that no nickel contaminated to the solid product (less than the limit of detection of ICP-MS, $<0.6 \mathrm{mg} \mathrm{Kg}^{-1}(<0.6 \mathrm{ppm})$ ).

\section{Reference}

1. Dastbaravardeh, N.; Schnürch, M.; Mihovilovic, M. D. Ruthenium(0)-catalyzed $\mathrm{sp}^{3} \mathrm{C}-\mathrm{H}$ bond arylation of benzylic amines using arylboronates. Org. Lett. 2012, 14, 1930.

2. Lew, T. T. S.; Lim, D. S. W.; Zhang, Y. Copper(I)-catalyzed amidation reaction of organoboronic esters and isocyanates. Green Chem. 2015, 17, 5140.

3. Ravel, B.; Newville, M. ATHENA, ARTEMIS, HEPHAESTUS: data analysis for X-ray absorption spectroscopy using IFEFFIT. J. Synchrotron Rad. 2005, 12, 537.

4. Zabinsky, S. I.; Rehr, J. J.; Ankudinov, A.; Albers, R. C.; Eller, M. J. Multiple-scattering calculations of X-ray absorption spectra. Phys. Rev. B 1995, 52, 2995.

5. Neese, F. The ORCA program system. WIRs Comput. Mol. Sci. 2012, 2, 73.

6. Grimme, S.; Ehrlich, S.; Goerigk, L. Effect of the damping function in dispersion corrected density functional theory. J. Comput. Chem. 2011, 32, 1456.

7. Grimme, S.; Antony, J.; Ehrlich, S.; Krieg, H. A consistent and accurate ab initio parametrization of density functional dispersion correction (DFT-D) for the 94 elements H-Pu. J. Chem. Phys. 2010, 132, 154104.

8. He, Z.-T.; Hartwig, J. F. Palladium-catalyzed $\alpha$-arylation of carboxylic acids and secondary amides via a traceless protecting strategy. J. Am. Chem. Soc. 2019, 141, 11749.

9. Li, G.; Szostak, M. Highly selective transition-metal-free transamidation of amides and amidation of esters at room temperature. Nat. Commun. 2018, 9, 4165 .

10. Zhang, G.; Zhao, Y.; Xuan, L.; Ding, C. $\mathrm{SO}_{2} \mathrm{~F}_{2}$-activated efficient Beckmann rearrangement of ketoximes for accessing amides and lactams. Eur. J. Org. Chem. 2019, 4911.

11. Yuan, Y.-C.; Kamaraj, R.; Bruneau, C.; Labasque, T.; Roisnel, T. Unmasking amides: Ruthenium-catalyzed protodecarbonylation of $N$-substituted phthalimide derivatives. Org. Lett. 2017, 19, 6404.

12. Fu, W. C.; Jamison, T. F. Modular continuous flow synthesis of imatinib and analogues. Org. Lett. 2019, 21, 6112.

13. Shen, N.; Cheung, C. W.; Ma, J.-A. Direct amide synthesis via Ni-mediated aminocarbonylation of arylboronic acids with CO and nitroarenes. Chem. Commun. 2019, 55, 13709. 
14. De, S.; Yin, J.; Ma, D. Copper-catalyzed coupling reaction of (hetero)aryl chlorides and amides. Org. Lett. 2017, 19, 4864.

15. Du, J.; Luo, K.; Zhang, X. Synthesis of amides through an oxidative amidation of tetrazoles with aldehydes under transition-metal-free conditions. RSC Adv. 2014, 4, 54539.

16. Yu, S.; Shin, T.; Zhang, M.; Xia, Y.; Kim, Y.; Lee, S. Nickel/briphos-catalyzed direct transamidation of unactivated secondary amides using trimethylsilyl chloride. Org. Lett. 2018, 20, 7563.

17. Ling, L.; Chen, C.; Luo, M.; Zeng, X. Chromium-catalyzed activation of acyl C-O bonds with magnesium for amidation of esters with nitroarenes. Org. Lett. 2019, 21, 1912.

18. Wang, S.-M.; Zhao, Z. X.; Qin, H.-L. Clickable coupling of carboxylic acids and amines at room temperature mediated by $\mathrm{SO}_{2} \mathrm{~F}_{2}$ : a significant breakthrough for the construction of amides and peptide linkages. Org. Biomol. Chem. 2019, 17, 4087.

19. Li, G.; Ji, C.-L.; Hong, X.; Szostak, M. Highly chemoselective, transition-metal-free transamidation of unactivated amides and direct amidation of alkyl esters by N-C/O-C cleavage. J. Am. Chem. Soc. 2019, 141, 11161.

20. Ghosh, T.; Jana, S.; Dash, J. KO ${ }^{t}$ Bu-promoted transition-metal-free transamidation of primary and tertiary amides with amines. Org. Lett. 2019, 21, 6690.

21. Shanmugam, K.; Ramdas, S.; Thirumanavelan, G. A straightforward metal-free and mild base promoted amidation and transesterification via acyl C-O bond cleavage-an expedite synthesis of aromatic amides and esters. ChemistrySelect 2019, 4, 175.

22. Bélanger, G.; April, M.; Dauphin, É.; Roy, S. Effect of substitution on the intramolecular 1,3-dipolar cycloaddition of alkene tethered Münchnones. J. Org. Chem. 2007, 72, 1104.

23. Nie, Q.; Yi, F.; Huang, B.; Cai, M. Efficient heterogeneous gold(I)-catalyzed direct C( $\left.\mathrm{sp}^{2}\right)-\mathrm{C}(\mathrm{sp})$ bond functionalization of arylalkynes through a nitrogenation process to amides. Adv. Synth. Catal. 2017, 359, 3968.

24. Chunchen, Y.; Ping, D.; Xiaoguang, B.; Yingsheng, Z. Highly site-selective formation of perfluoroalkylated anilids via a protecting strategy by molybdenum hexacarbonyl catalyst. Org. Lett. 2019, $21,6481$.

25. Wu, X.; Hu, L. Efficient amidation from carboxylic acids and azides via selenocarboxylates: application to the coupling of amino acids and peptides with azides. J. Org. Chem. 2007, 72, 765.

26. Li, G.; Ji, C.-L.; Hong, X.; Szostak, M. Highly chemoselective, transition-metal-free transamidation of unactivated amides and direct amidation of alkyl esters by N-C/O-C cleavage. J. Am. Chem. Soc. 2019, 141, 11161.

27. Zhou, T.; Ji, C.-K.; Hong, X.; Szostak, M. Palladium-catalyzed decarbonylative Suzuki-Miyaura crosscoupling of amides by carbon-nitrogen bond activation. Chem. Sci. 2019, 10, 9865.

28. Lee, J.; Hong, B.; Lee, A. Visible-light-promoted, catalyst-free Gomberg-Bachmann reaction: synthesis of biaryls. J. Org. Chem. 2019, 84, 9297. 
29. Saavedra, B.; González-Gallardo, N.; Meli, A.; Ramón, D. J. A bipyridine-palladium derivative as general precatalyst for cross-coupling reactions in deep eutectic solvents. Adv. Synth. Catal. 2019, 361, 3868.

30. Plaut, D. J.; Martin, S. M.; Kjaer, K.; Weygand, M. J.; Lahav, M., Leiserowitz, L.; Weissbuch, I.; Ward, M. D. Structural characterization of crystalline ternary inclusion compounds at the air-water interface. J. Am. Chem. Soc. 2003, 125, 15922.

31. Lu, S.-M.; Alper, H. Intramolecular carbonylation reactions with recyclable palladium-complexed dendrimers on silica: synthesis of oxygen, nitrogen, or sulfur-containing medium ring fused heterocycles. J. Am. Chem. Soc. 2005, 127, 14776.

32. Mali, S. M.; Bhaisare, R. D.; Gopi, H. N. Thioacids mediated selective and mild N-acylation of amines. J. Org. Chem. 2013, 78, 5550 .

33. Liao, L.-Y.; Liu, K.-M.; Duan, X.-F. Unified protocol for cobalt-catalyzed oxidative assembly of two aryl metal reagents using oxygen as an oxidant. J. Org. Chem. 2015, 80, 9856.

34. Mahadevi, A. S.; Sastry, G. N. Cation- $\pi$ interaction: its role and relevance in chemistry, biology, and material science. Chem. Rev. 2013, 113, 2100.

35. Liu, L.; Zhang, S.; Chen, H.; Lv, Y.; Zhu, J.; Zhao, Y. Mechanistic insight into the nickel-catalyzed crosscoupling of aryl phosphates with arylboronic acids: potassium phosphate is not a spectator base but is involved in the transmetalation step in the Suzuki-Miyaura reaction. Chem. Asian J. 2013, 8, 2592.

36. Liu, L.; Chen, P.; Sun, Y.; Wu, Y.; Chen, S.; Zhu, J.; Zhao, Y. Mechanism of nickel-catalyzed selective C-N bond activation in Suzuki-Miyaura cross-coupling of amides: a theoretical investigation. J. Org. Chem. 2016, $81,11686$.

37. Mikkelsen, K. V.; Ratner, M. A. Electron tunneling in solid-state electron-transfer reactions. Chem. Rev. 1987, 87,113 . 


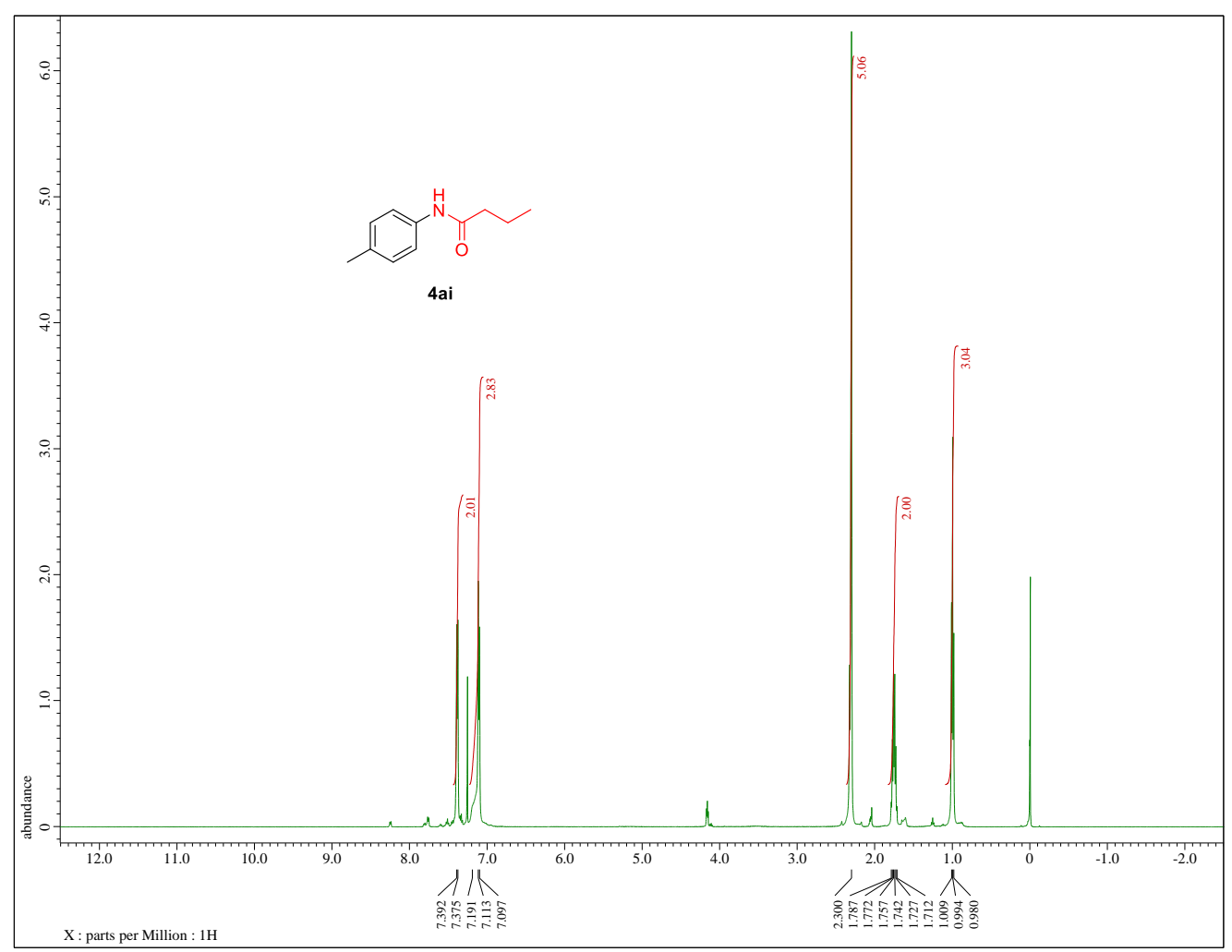

Figure 20-1. ${ }^{1} \mathrm{H}$ NMR spectrum of compound 4ai $\left(500 \mathrm{MHz}, \mathrm{CDCl}_{3}\right)$

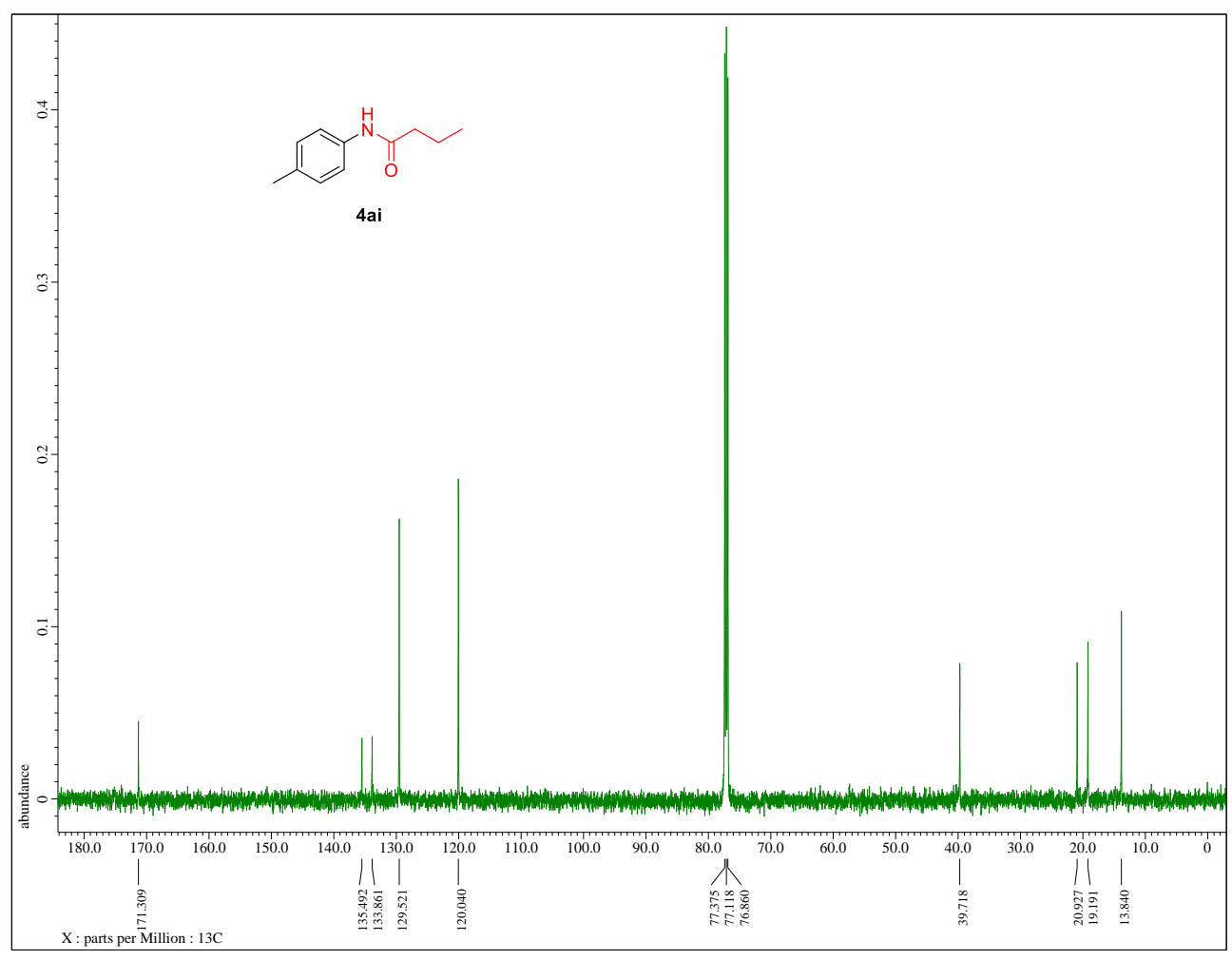

Figure S20-2. ${ }^{13} \mathrm{C}$ NMR spectrum of compound 4ai $\left(125 \mathrm{MHz}, \mathrm{CDCl}_{3}\right)$ 


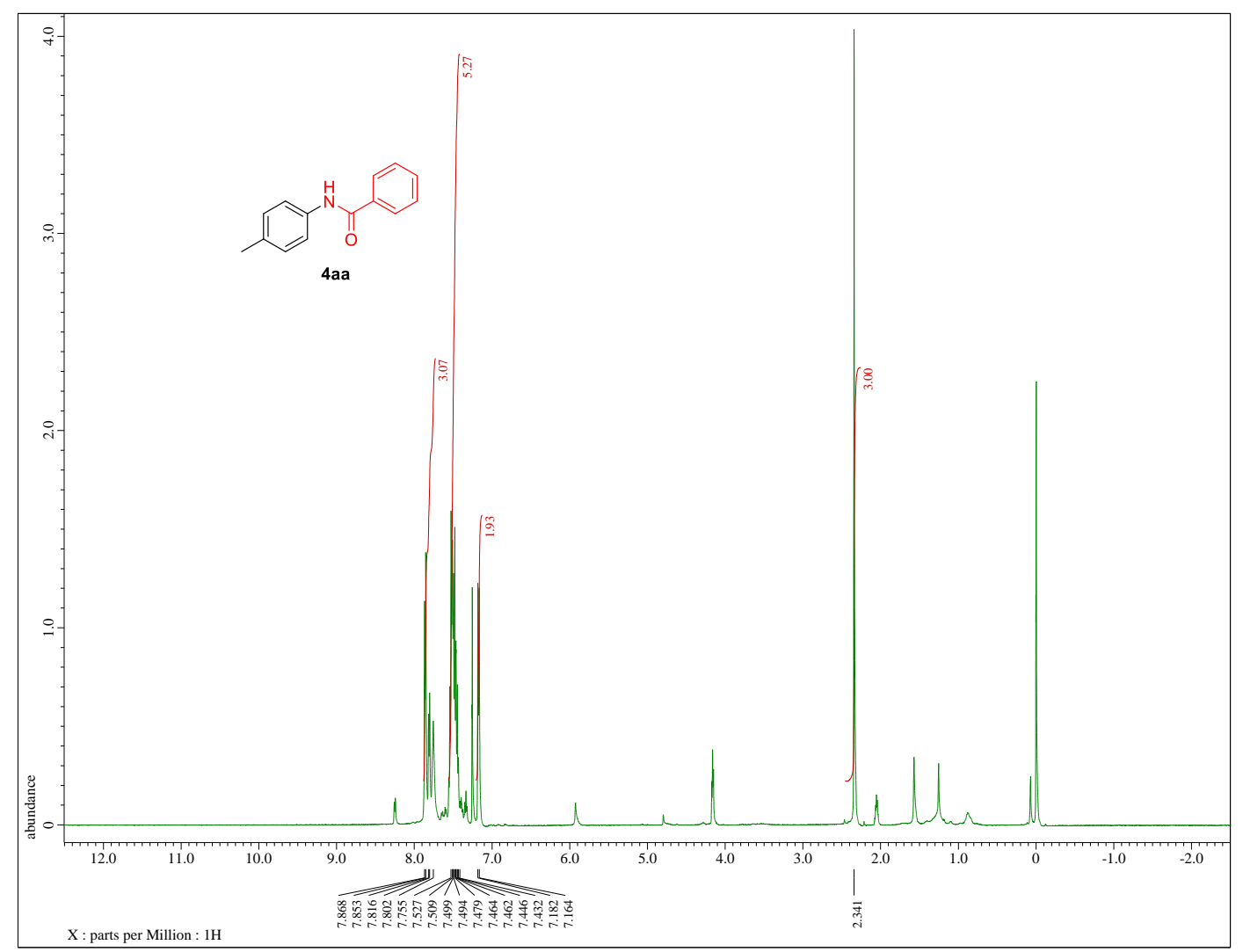

Figure S21-1. ${ }^{1} \mathrm{H}$ NMR spectrum of compound $4 \mathbf{a a}\left(500 \mathrm{MHz}, \mathrm{CDCl}_{3}\right)$

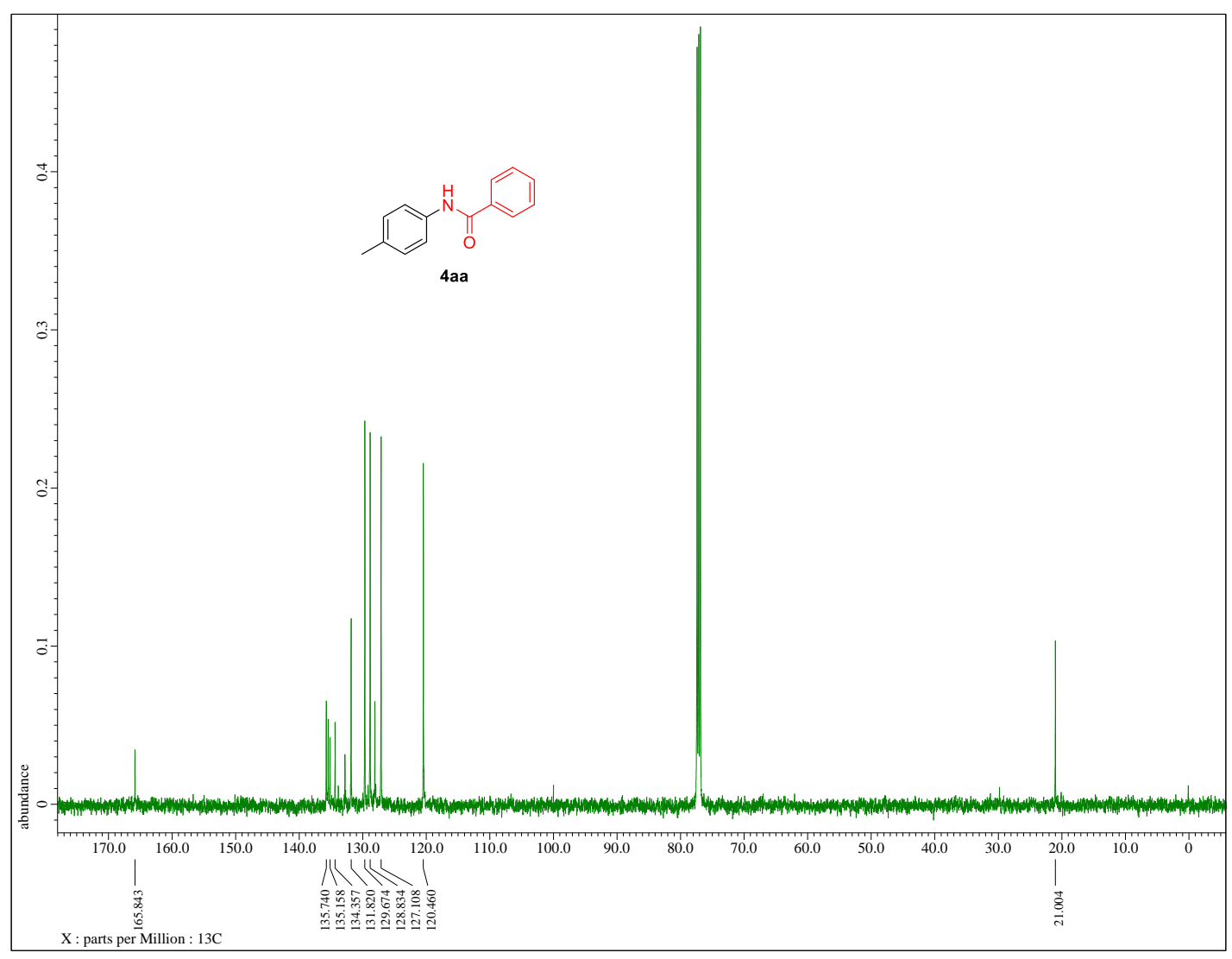

Figure S21-2. ${ }^{13} \mathrm{C}$ NMR spectrum of compound 4aa $\left(125 \mathrm{MHz}, \mathrm{CDCl}_{3}\right)$ 


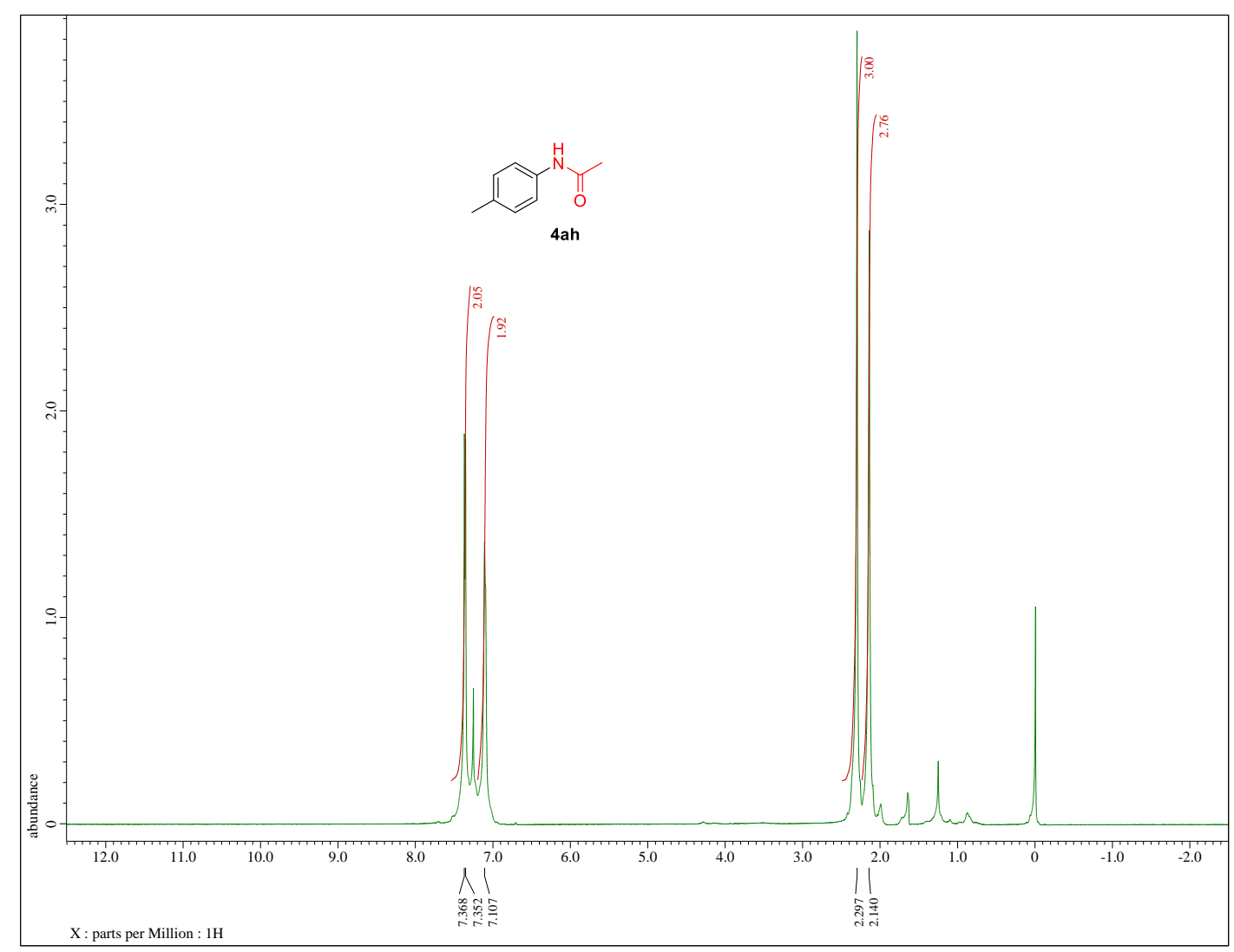

Figure S22-1. ${ }^{1} \mathrm{H}$ NMR spectrum of compound $\mathbf{4 a h}\left(500 \mathrm{MHz}, \mathrm{CDCl}_{3}\right)$

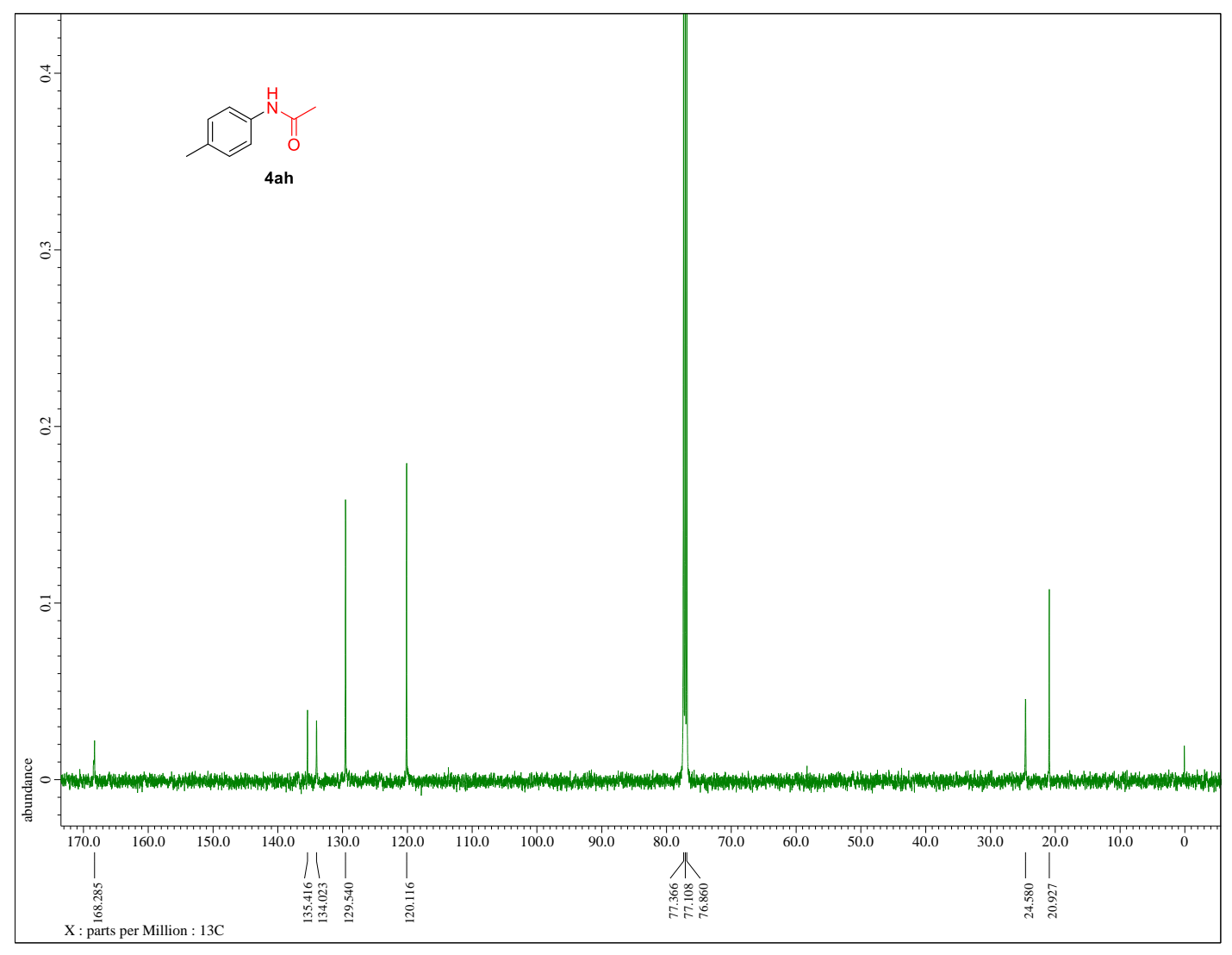

Figure S22-2. ${ }^{13} \mathrm{C}$ NMR spectrum of compound 4ah $\left(125 \mathrm{MHz}, \mathrm{CDCl}_{3}\right)$ 


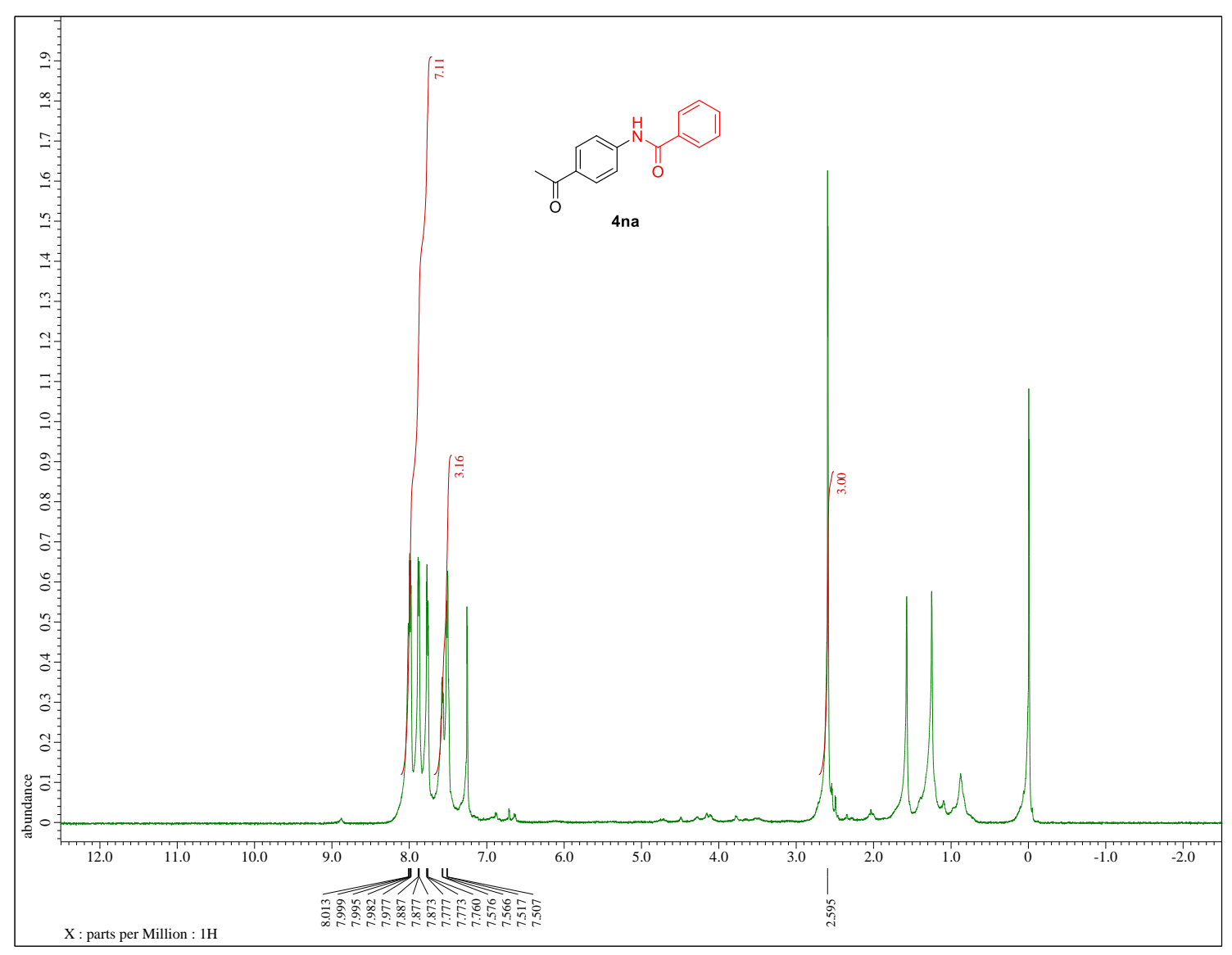

Figure S23-1. ${ }^{1} \mathrm{H}$ NMR spectrum of compound 4na $\left(500 \mathrm{MHz}, \mathrm{CDCl}_{3}\right)$

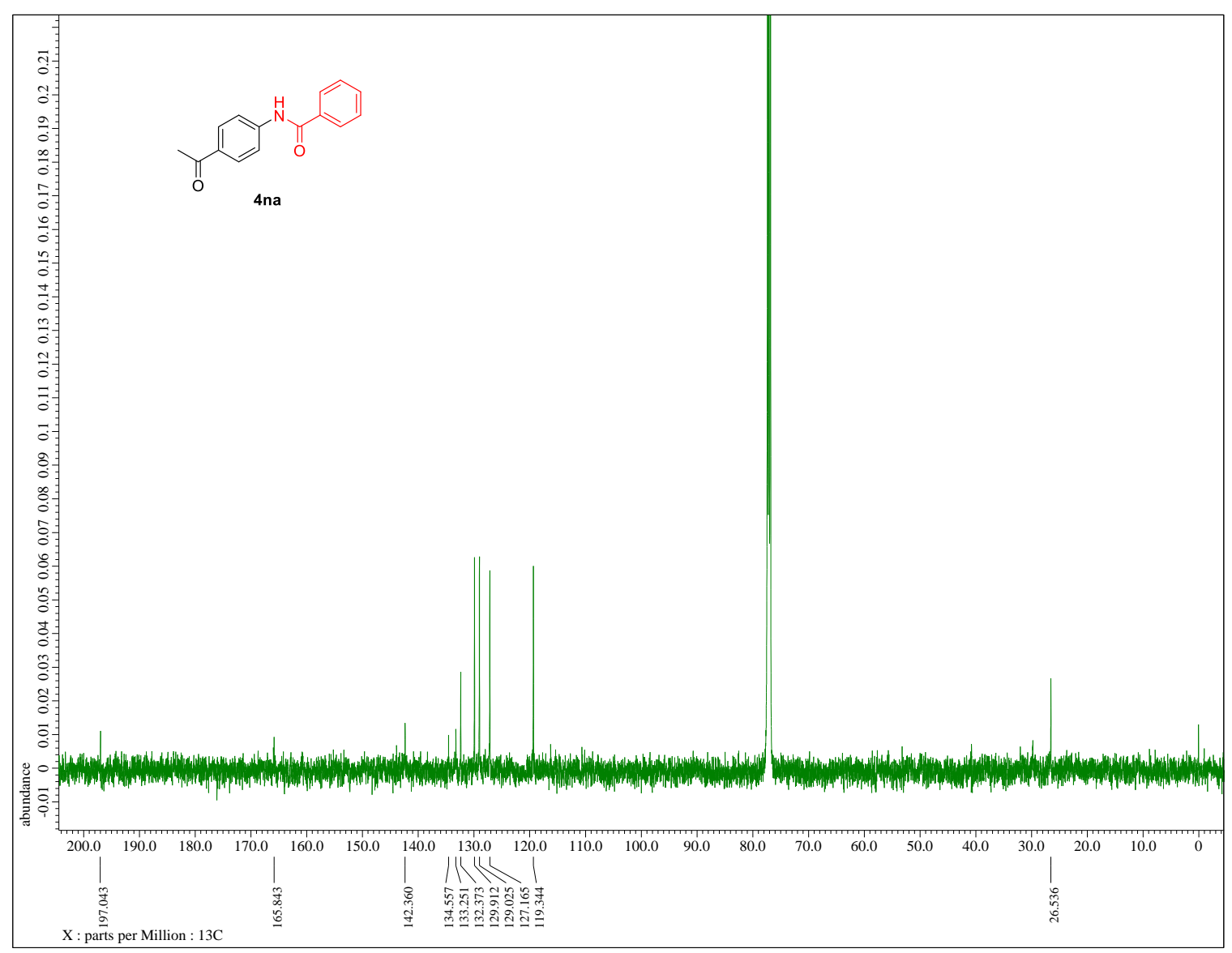

Figure S23-2. ${ }^{13} \mathrm{C}$ NMR spectrum of compound 4 na $\left(125 \mathrm{MHz}, \mathrm{CDCl}_{3}\right)$ 


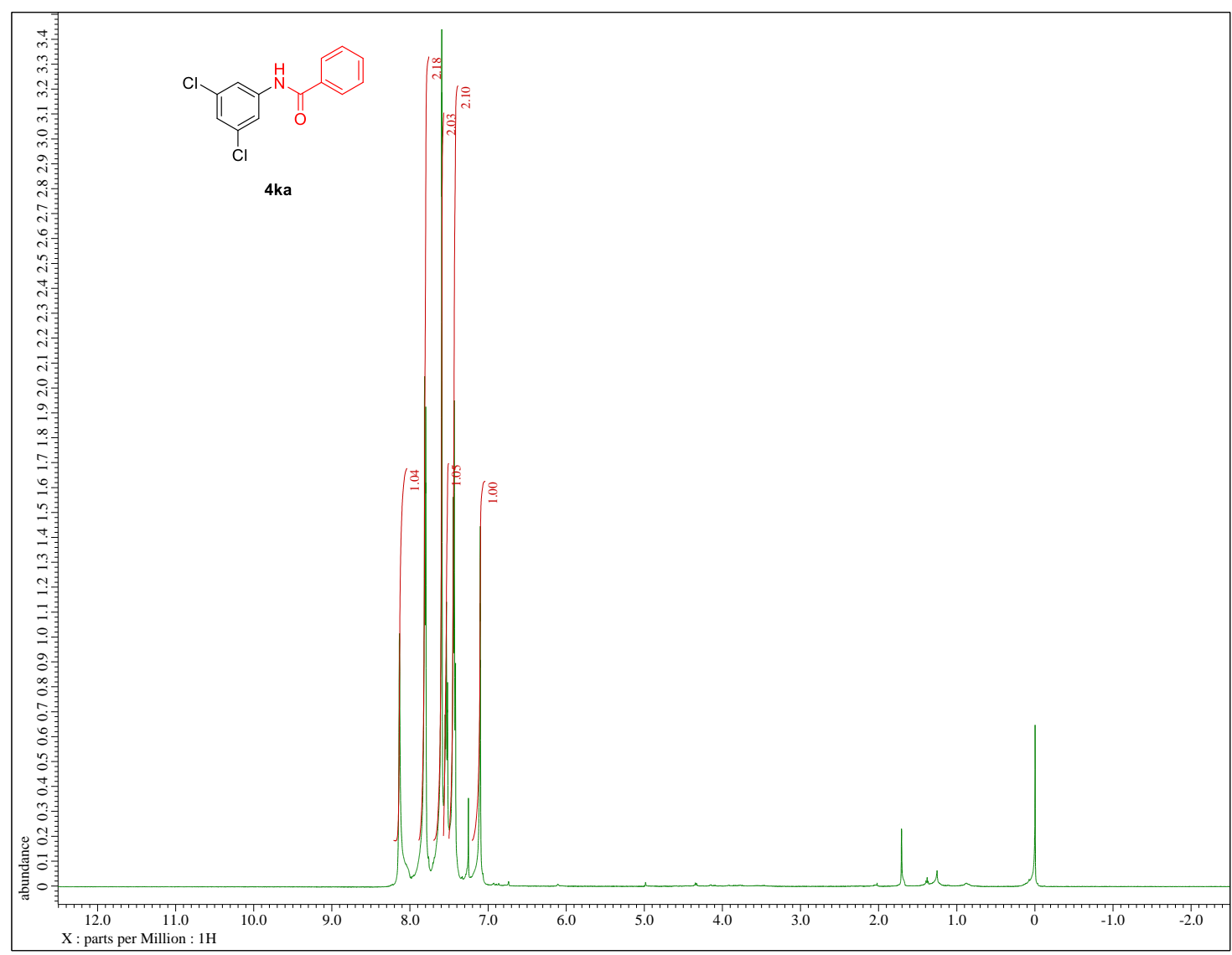

Figure S24-1. ${ }^{1} \mathrm{H}$ NMR spectrum of compound $4 \mathbf{k a}\left(500 \mathrm{MHz}, \mathrm{CDCl}_{3}\right)$

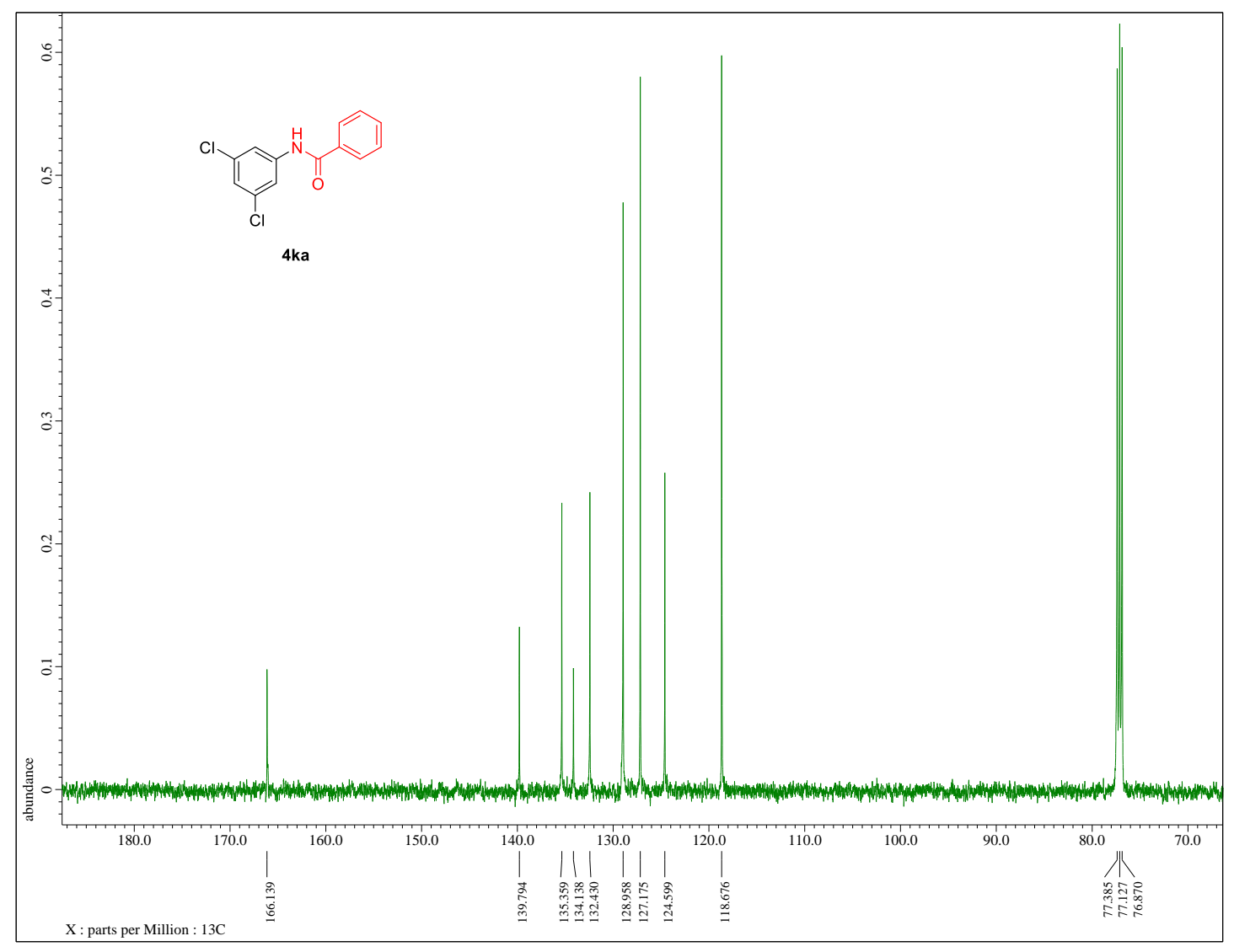

Figure S24-2. ${ }^{13} \mathrm{C}$ NMR spectrum of compound $\mathbf{4 k a}\left(125 \mathrm{MHz}, \mathrm{CDCl}_{3}\right)$ 


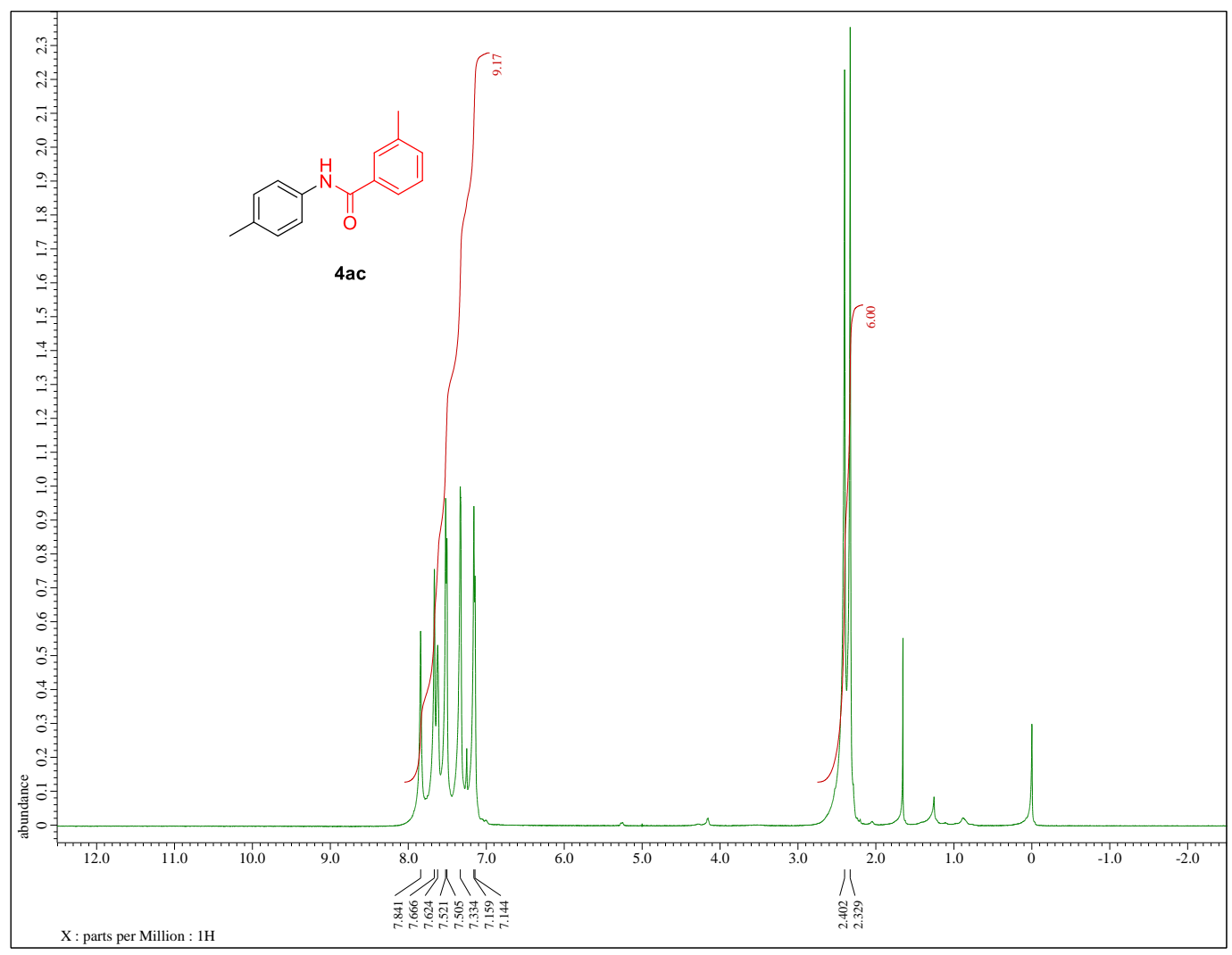

Figure S25-1. ${ }^{1} \mathrm{H}$ NMR spectrum of compound $4 \mathbf{a c}\left(500 \mathrm{MHz}, \mathrm{CDCl}_{3}\right)$

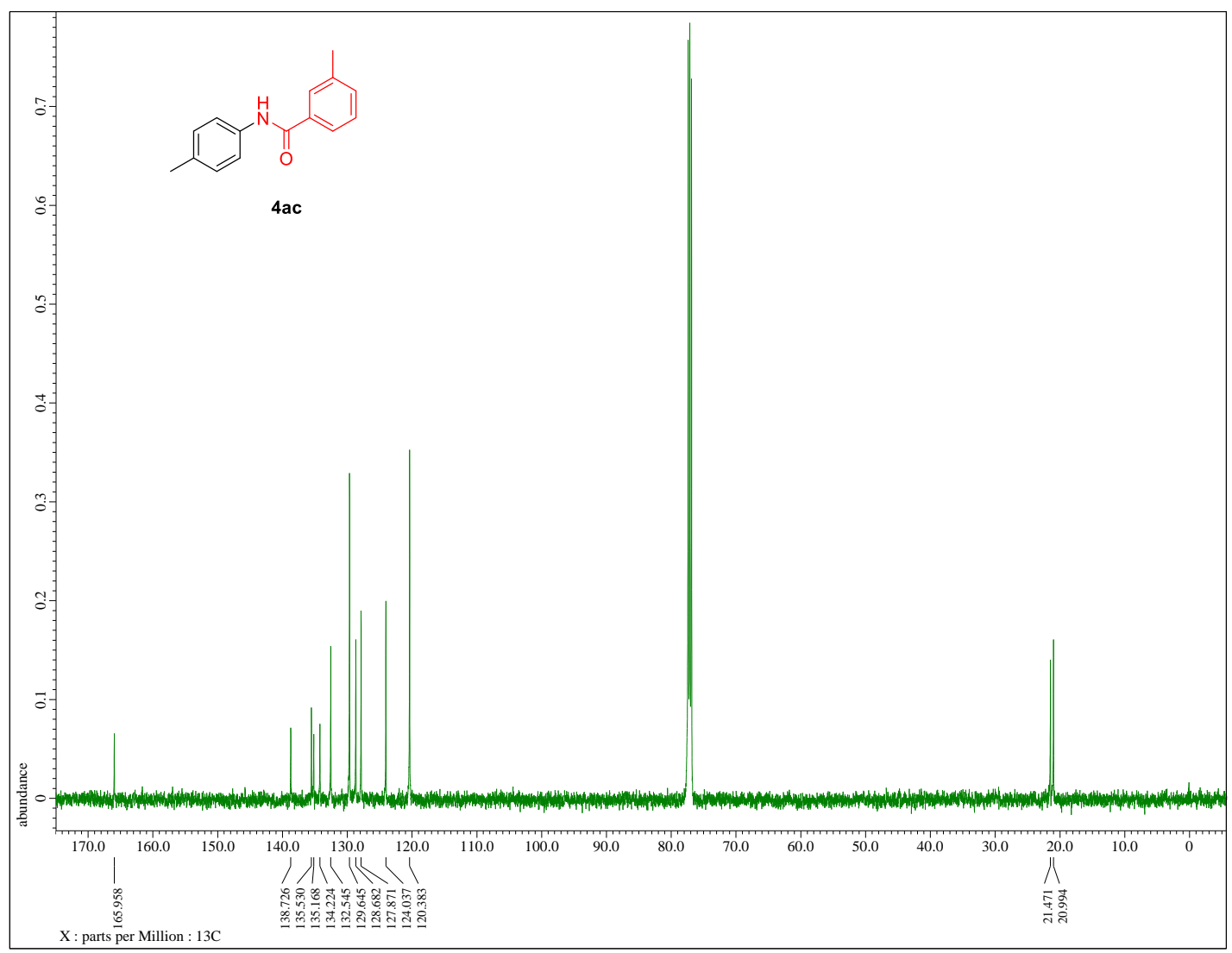

Figure S25-2. ${ }^{13} \mathrm{C}$ NMR spectrum of compound 4 ac $\left(125 \mathrm{MHz}, \mathrm{CDCl}_{3}\right)$ 


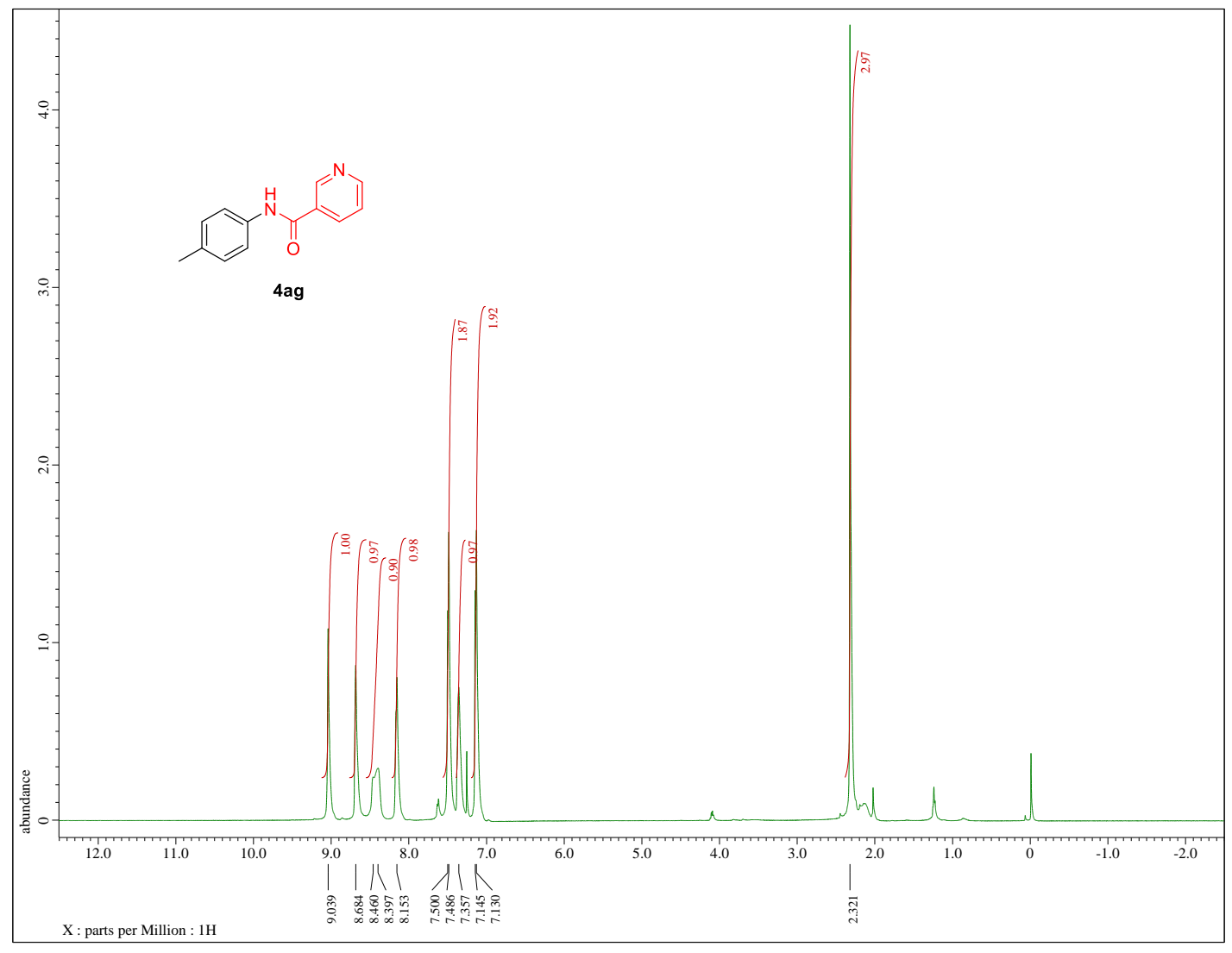

Figure S26-1. ${ }^{1} \mathrm{H}$ NMR spectrum of compound $4 \mathbf{a g}\left(500 \mathrm{MHz}, \mathrm{CDCl}_{3}\right)$

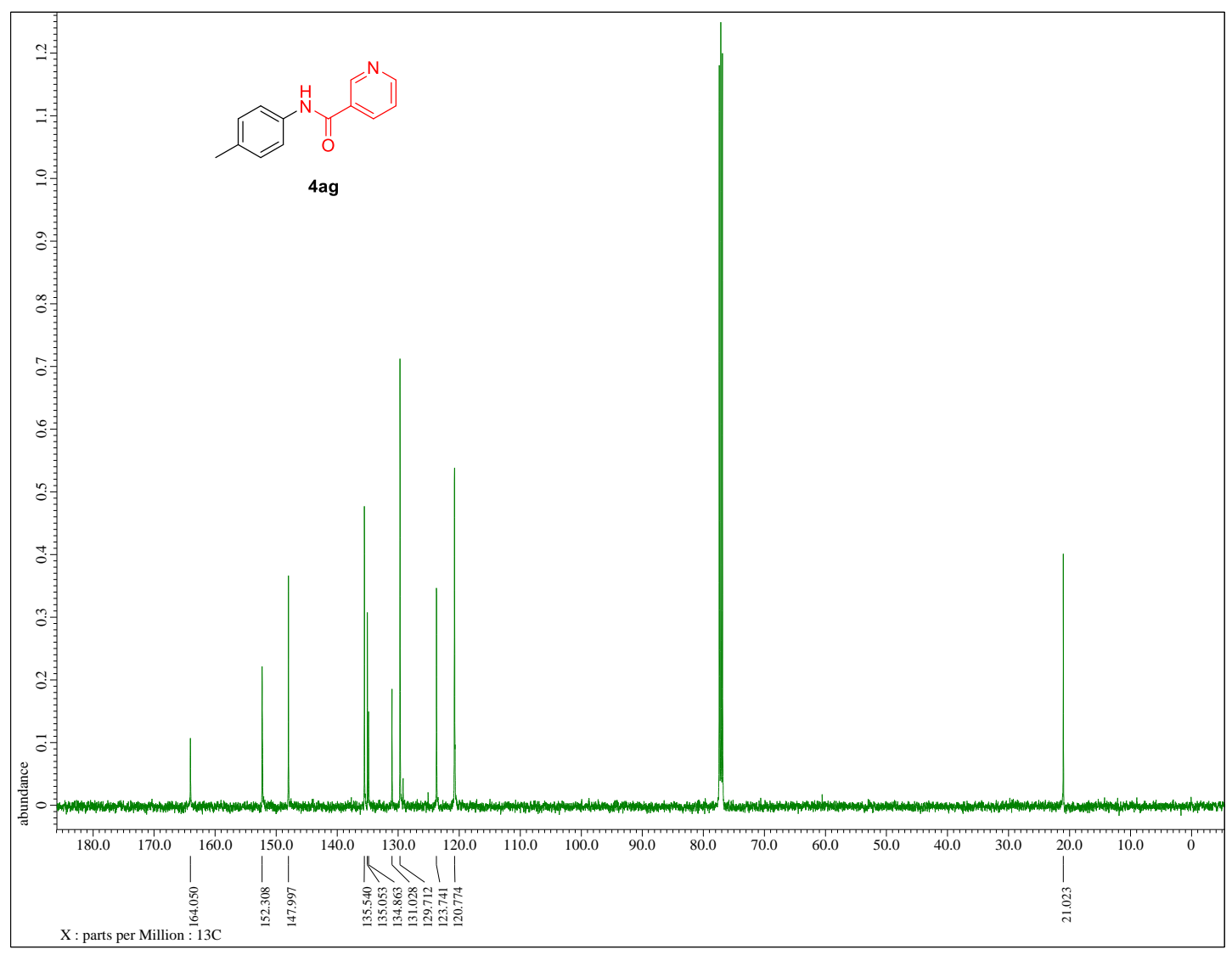

Figure S26-2. ${ }^{13} \mathrm{C}$ NMR spectrum of compound 4ag $\left(125 \mathrm{MHz}, \mathrm{CDCl}_{3}\right)$ 


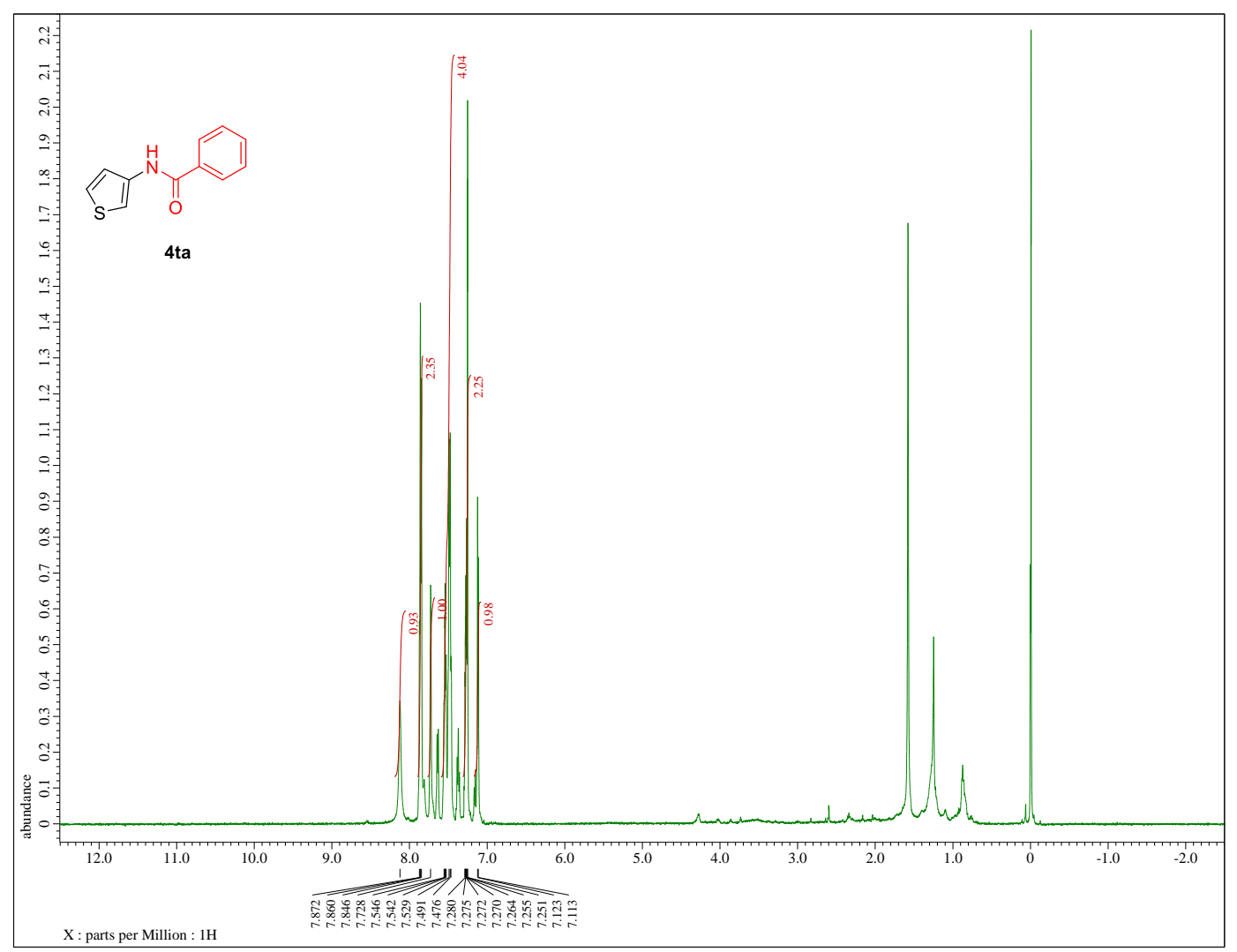

Figure S27-1. ${ }^{1} \mathrm{H}$ NMR spectrum of compound 4 ta $\left(500 \mathrm{MHz}, \mathrm{CDCl}_{3}\right)$

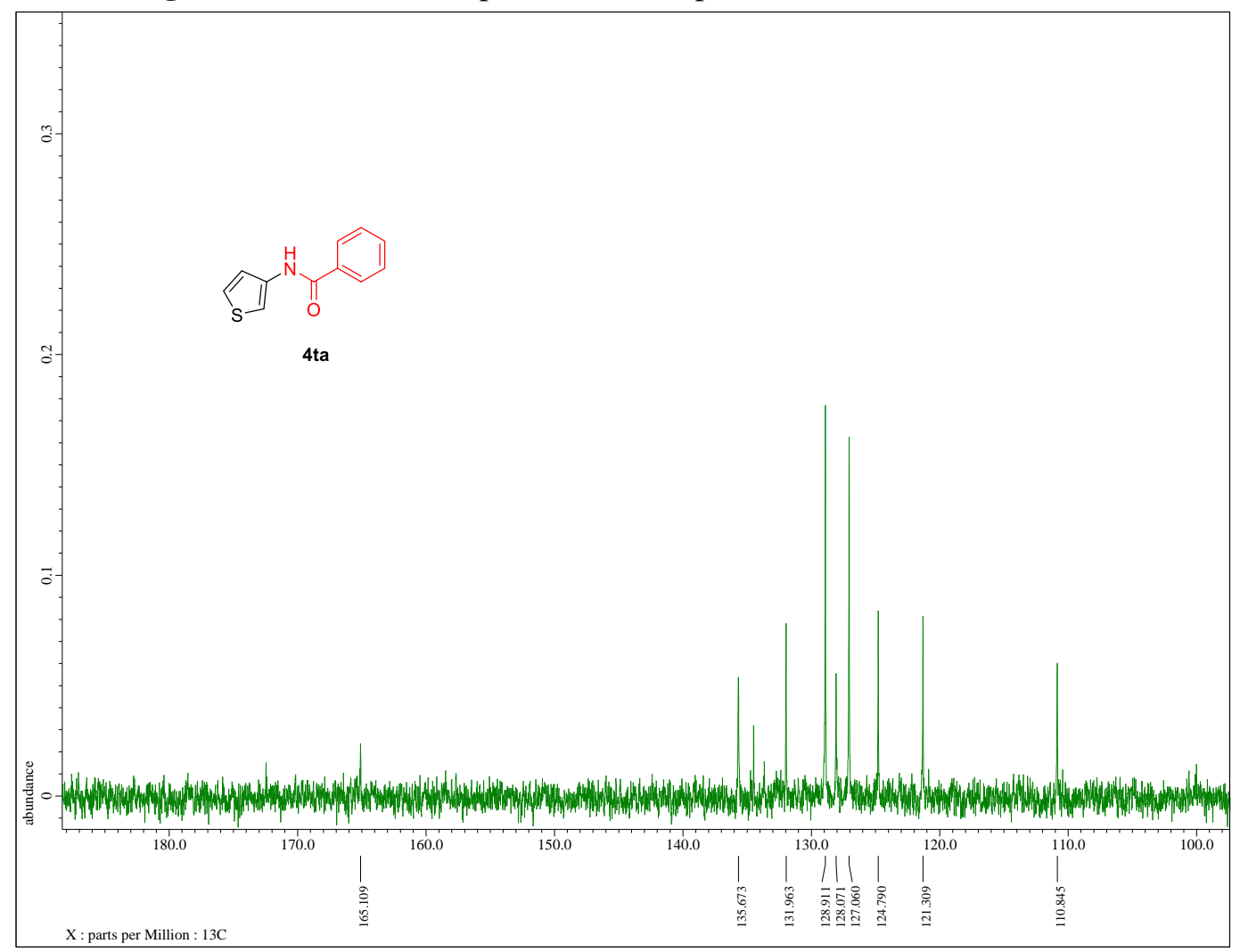

Figure S27-2. ${ }^{13} \mathrm{C}$ NMR spectrum of compound 4 ta $\left(125 \mathrm{MHz}, \mathrm{CDCl}_{3}\right)$ 


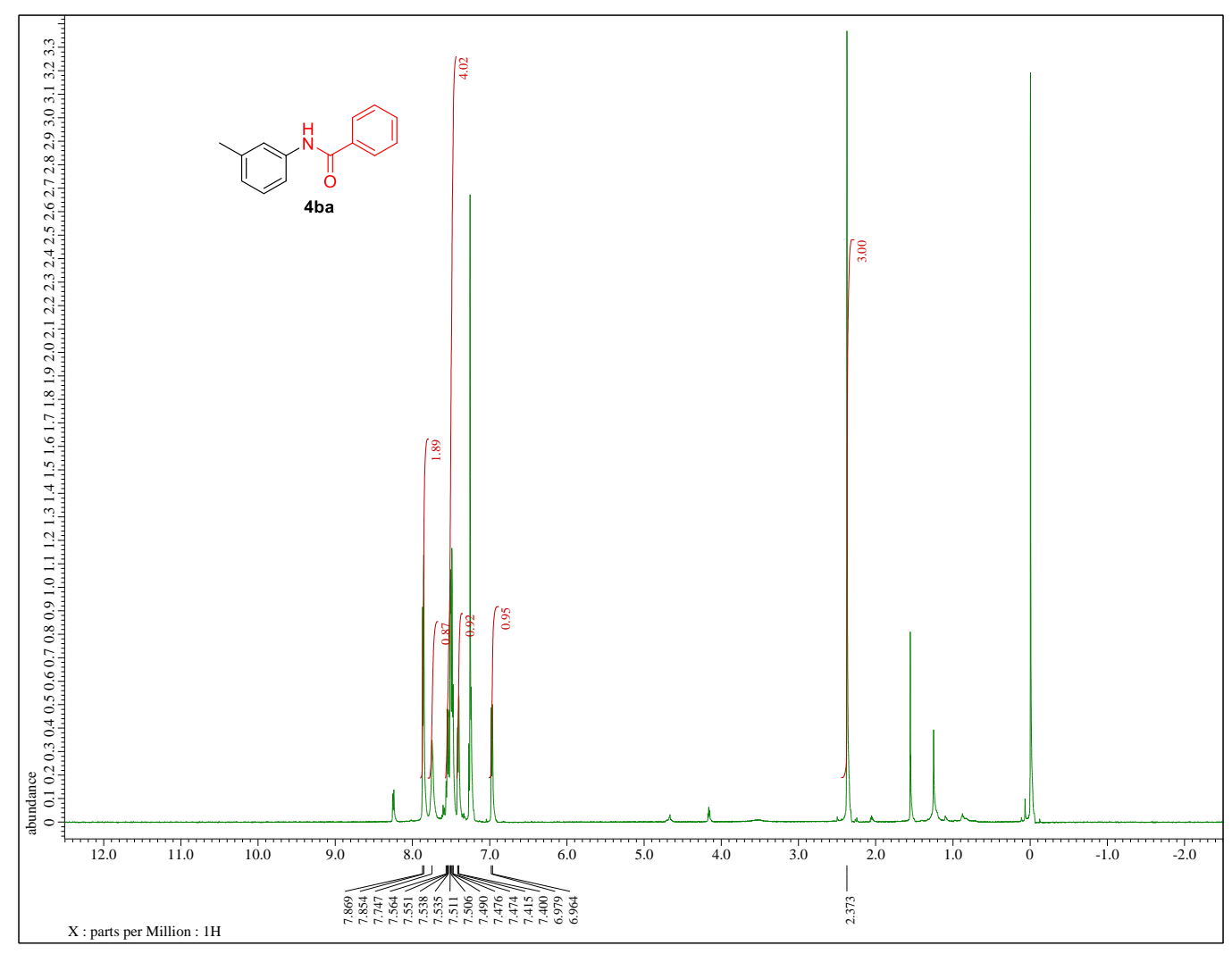

Figure S28-1. ${ }^{1} \mathrm{H}$ NMR spectrum of compound 4 ba $\left(500 \mathrm{MHz}, \mathrm{CDCl}_{3}\right)$

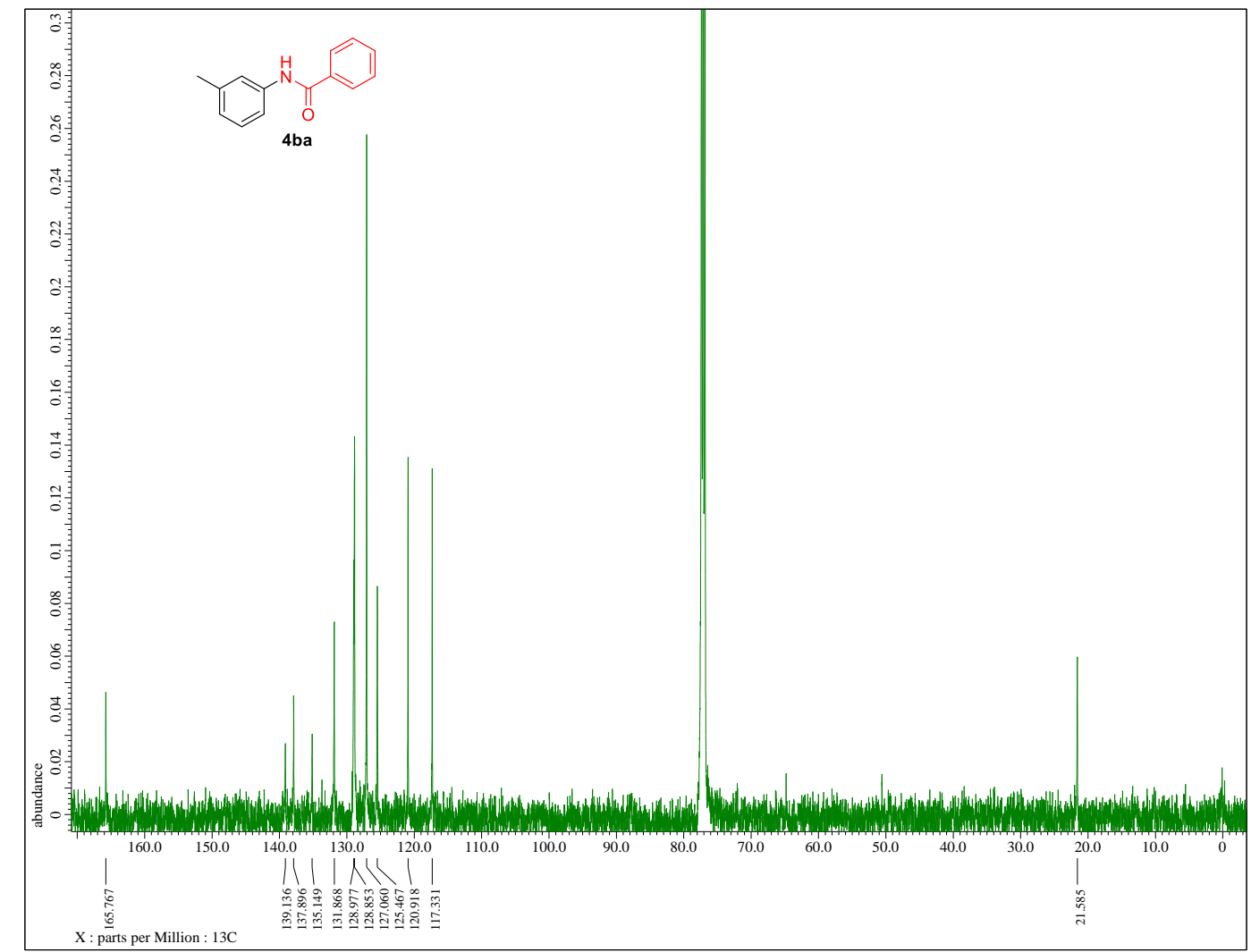

Figure S28-2. ${ }^{13} \mathrm{C}$ NMR spectrum of compound 4 ba $\left(125 \mathrm{MHz}, \mathrm{CDCl}_{3}\right)$ 


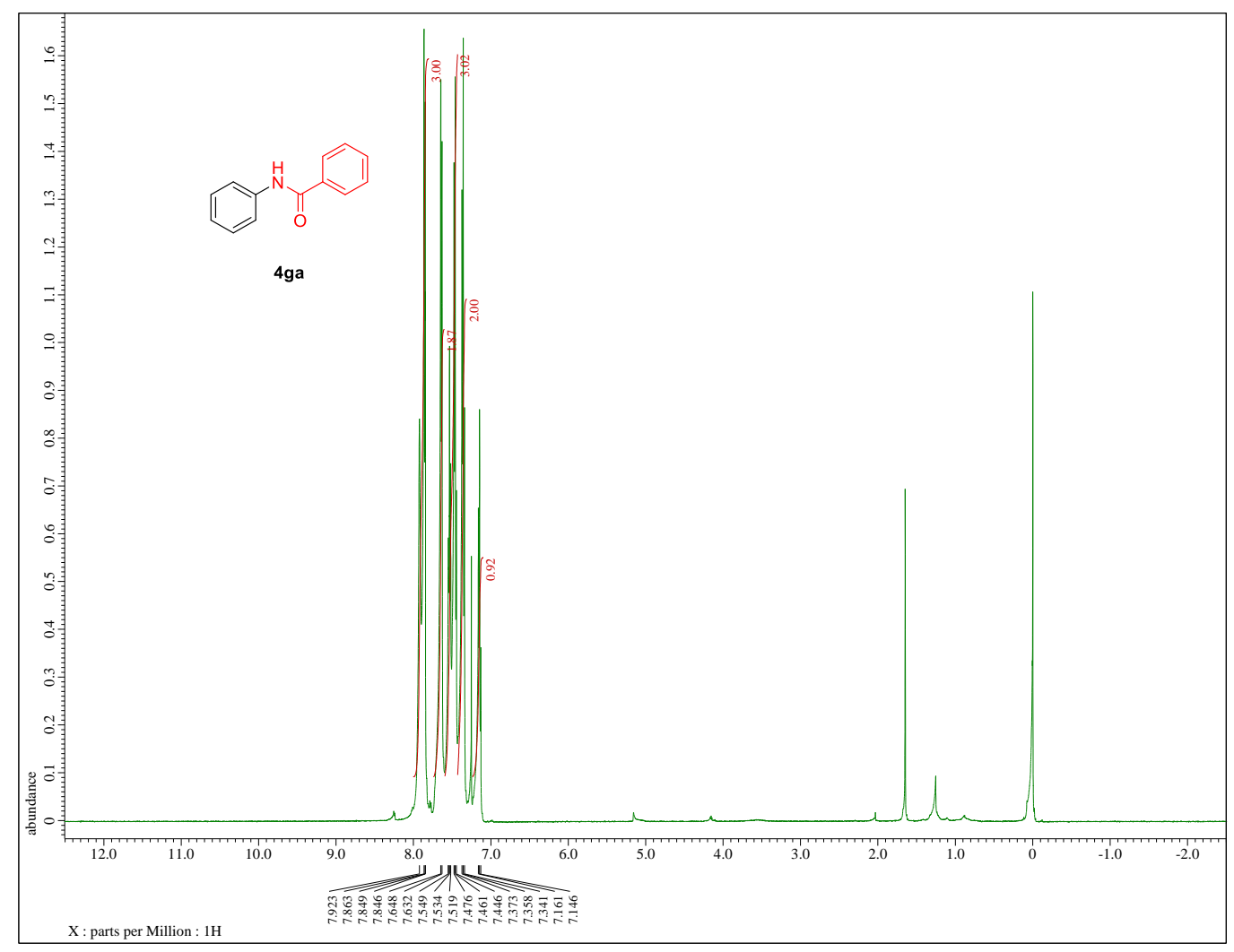

Figure S29-1. ${ }^{1} \mathrm{H}$ NMR spectrum of compound 4 ga $\left(500 \mathrm{MHz}, \mathrm{CDCl}_{3}\right)$

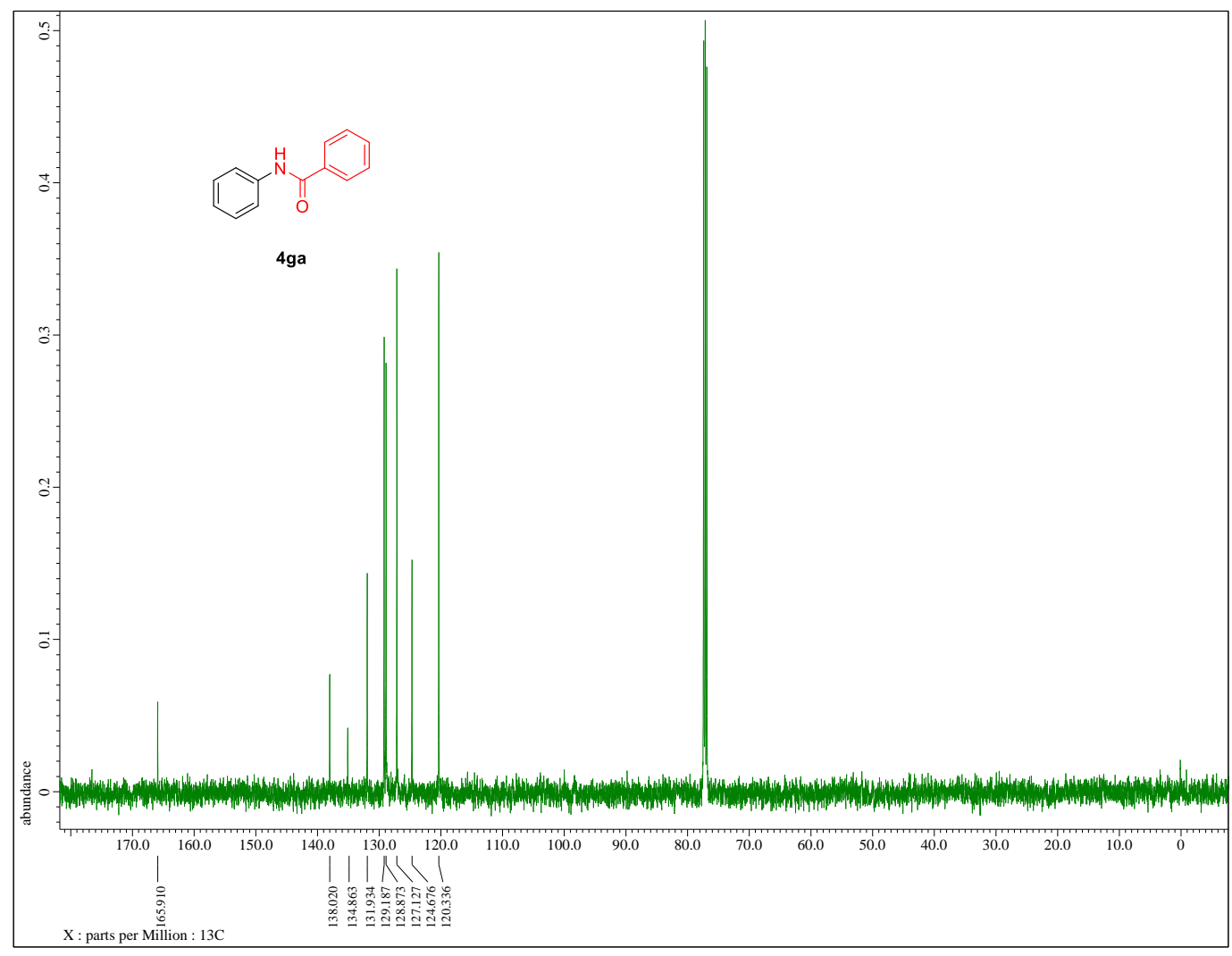

Figure S29-2. ${ }^{13} \mathrm{C}$ NMR spectrum of compound 4 ga $\left(125 \mathrm{MHz}, \mathrm{CDCl}_{3}\right)$ 


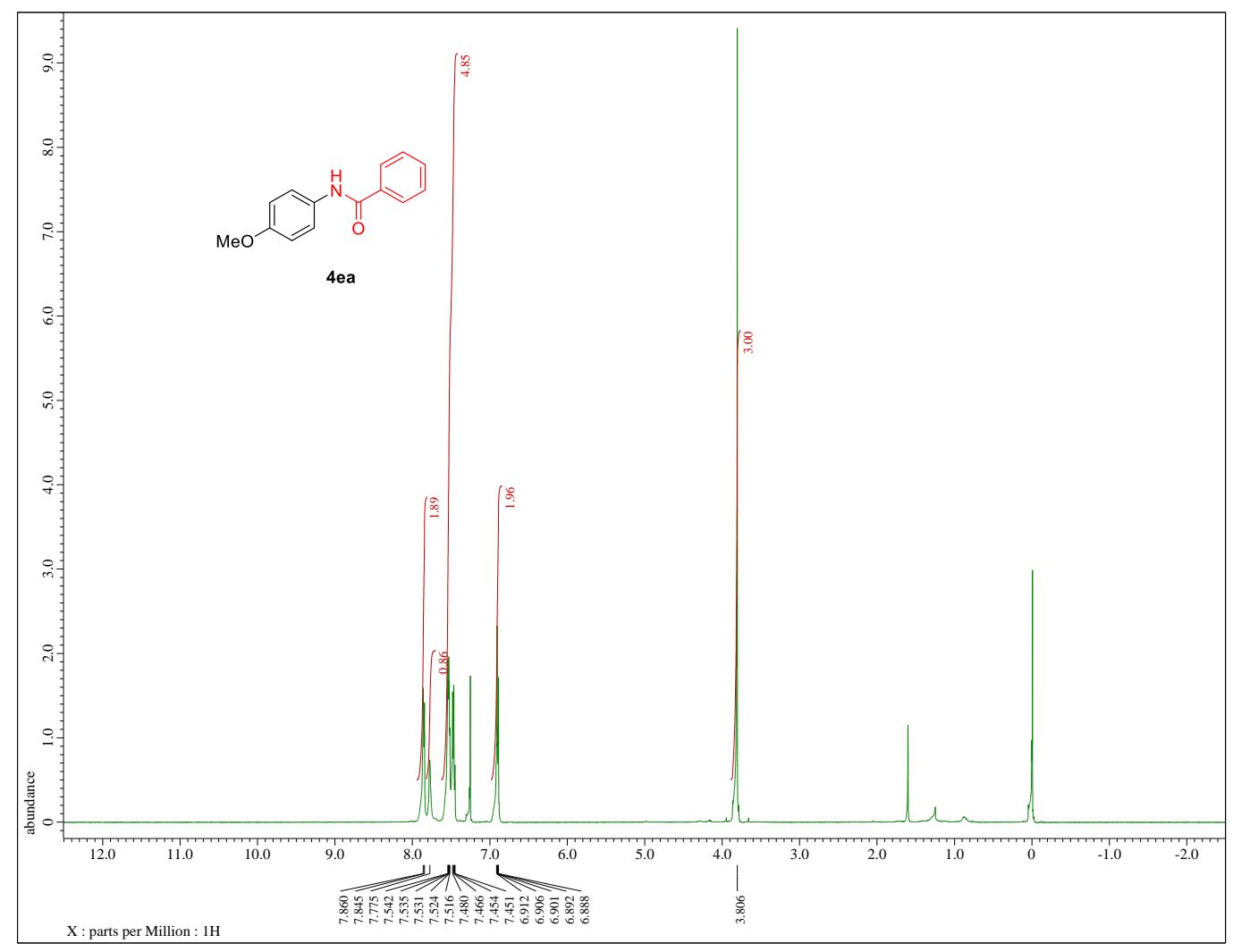

Figure S30-1. ${ }^{1} \mathrm{H}$ NMR spectrum of compound $4 \mathbf{e a ~}\left(500 \mathrm{MHz}, \mathrm{CDCl}_{3}\right)$

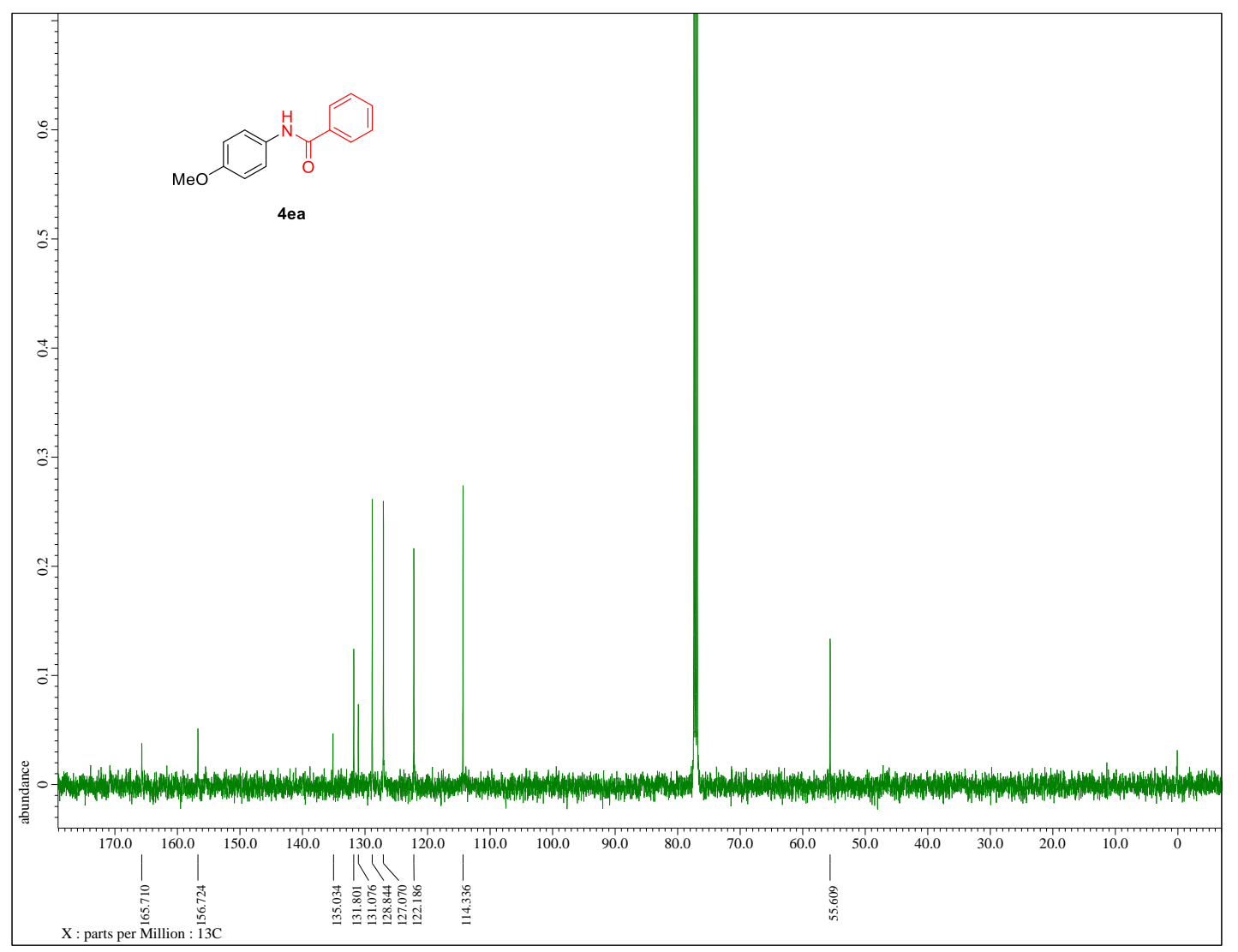

Figure S30-2. ${ }^{13} \mathrm{C}$ NMR spectrum of compound 4 ea $\left(125 \mathrm{MHz}, \mathrm{CDCl}_{3}\right)$ 


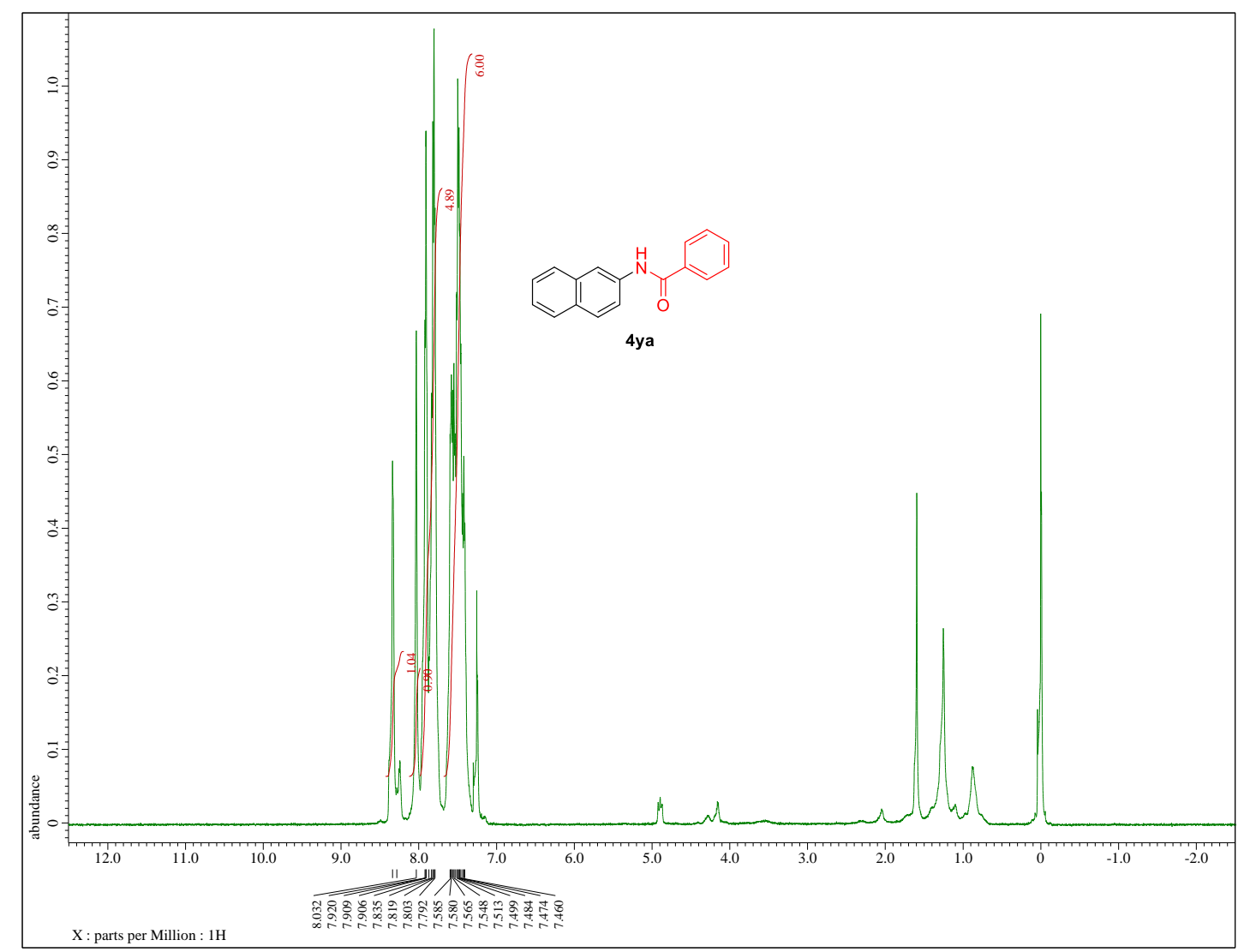

Figure S31-1. ${ }^{1} \mathrm{H}$ NMR spectrum of compound $4 \mathbf{y a}\left(500 \mathrm{MHz}, \mathrm{CDCl}_{3}\right)$

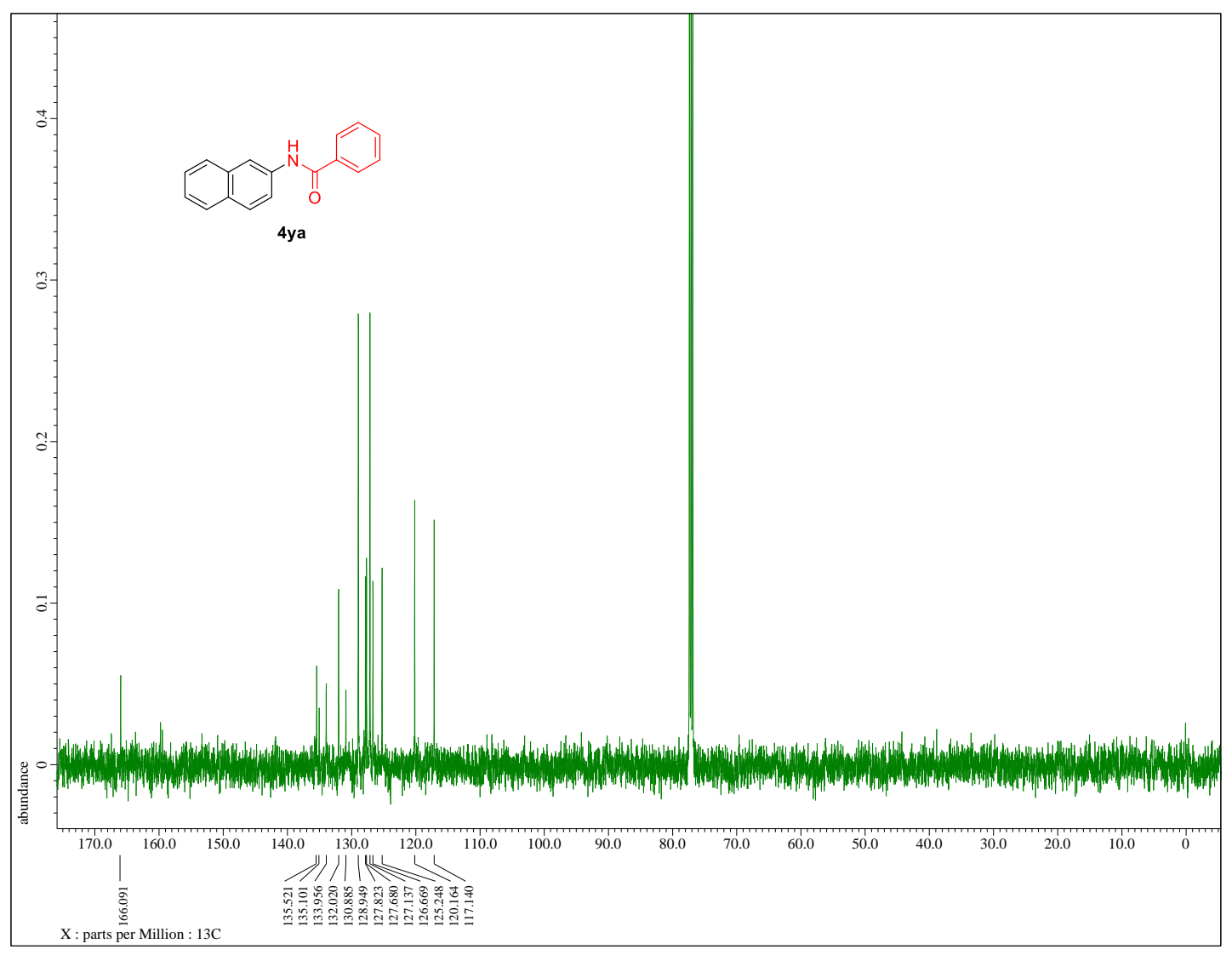

Figure S31-2. ${ }^{13} \mathrm{C}$ NMR spectrum of compound 4 ya $\left(125 \mathrm{MHz}, \mathrm{CDCl}_{3}\right)$ 


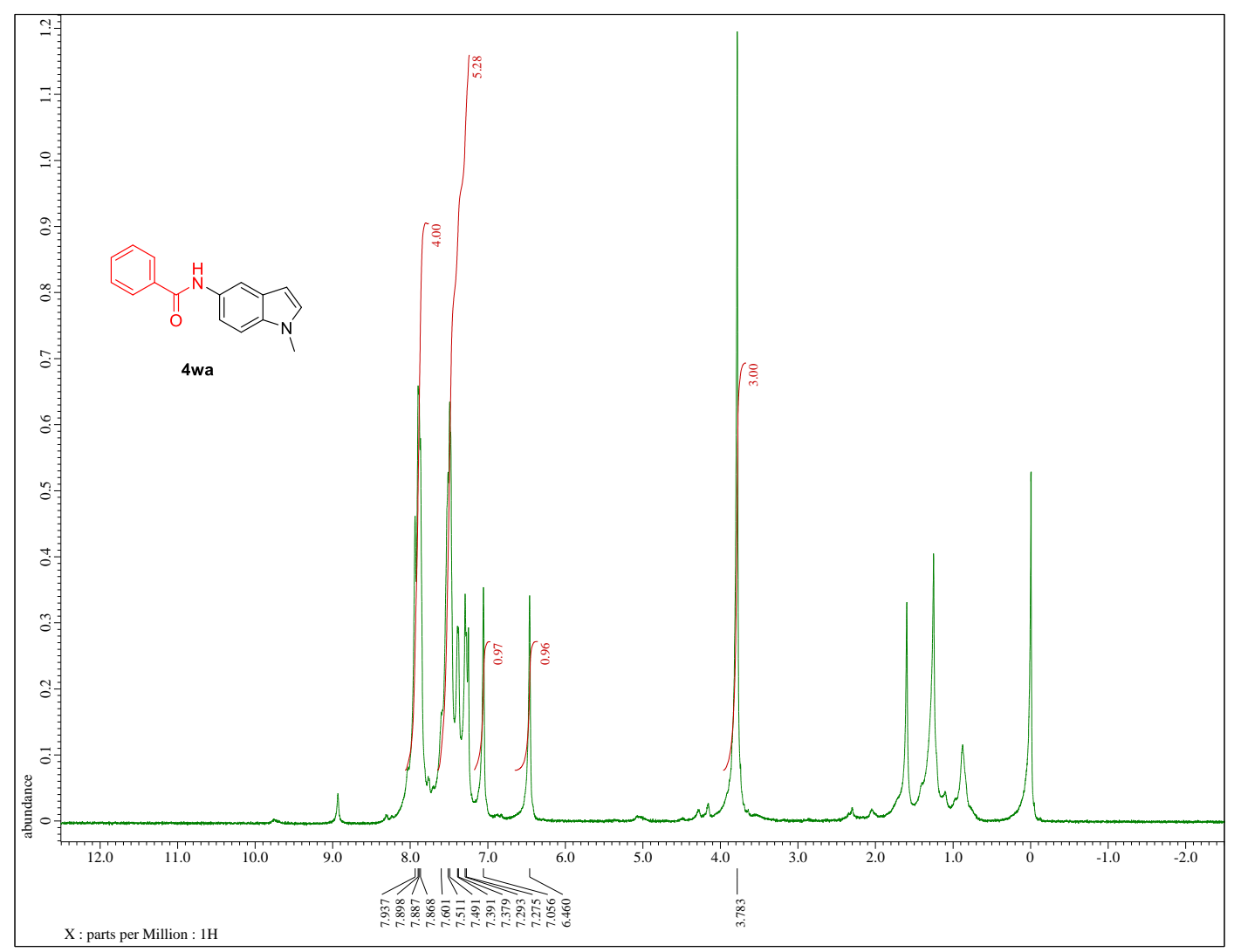

Figure S32-1. ${ }^{1} \mathrm{H}$ NMR spectrum of compound 4 wa $\left(500 \mathrm{MHz}, \mathrm{CDCl}_{3}\right)$

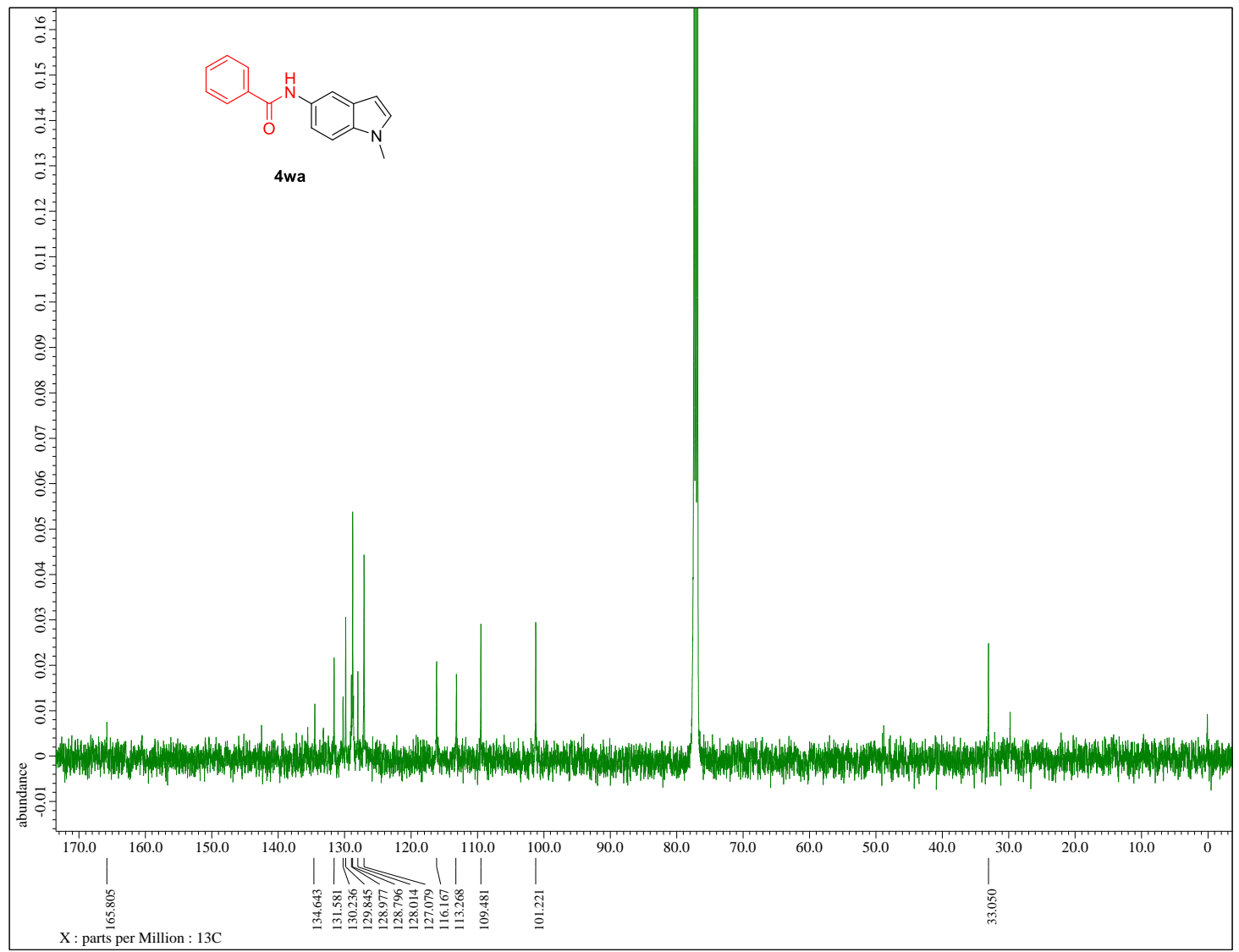

Figure S32-2. ${ }^{13} \mathrm{C}$ NMR spectrum of compound 4 wa $\left(125 \mathrm{MHz}, \mathrm{CDCl}_{3}\right)$ 


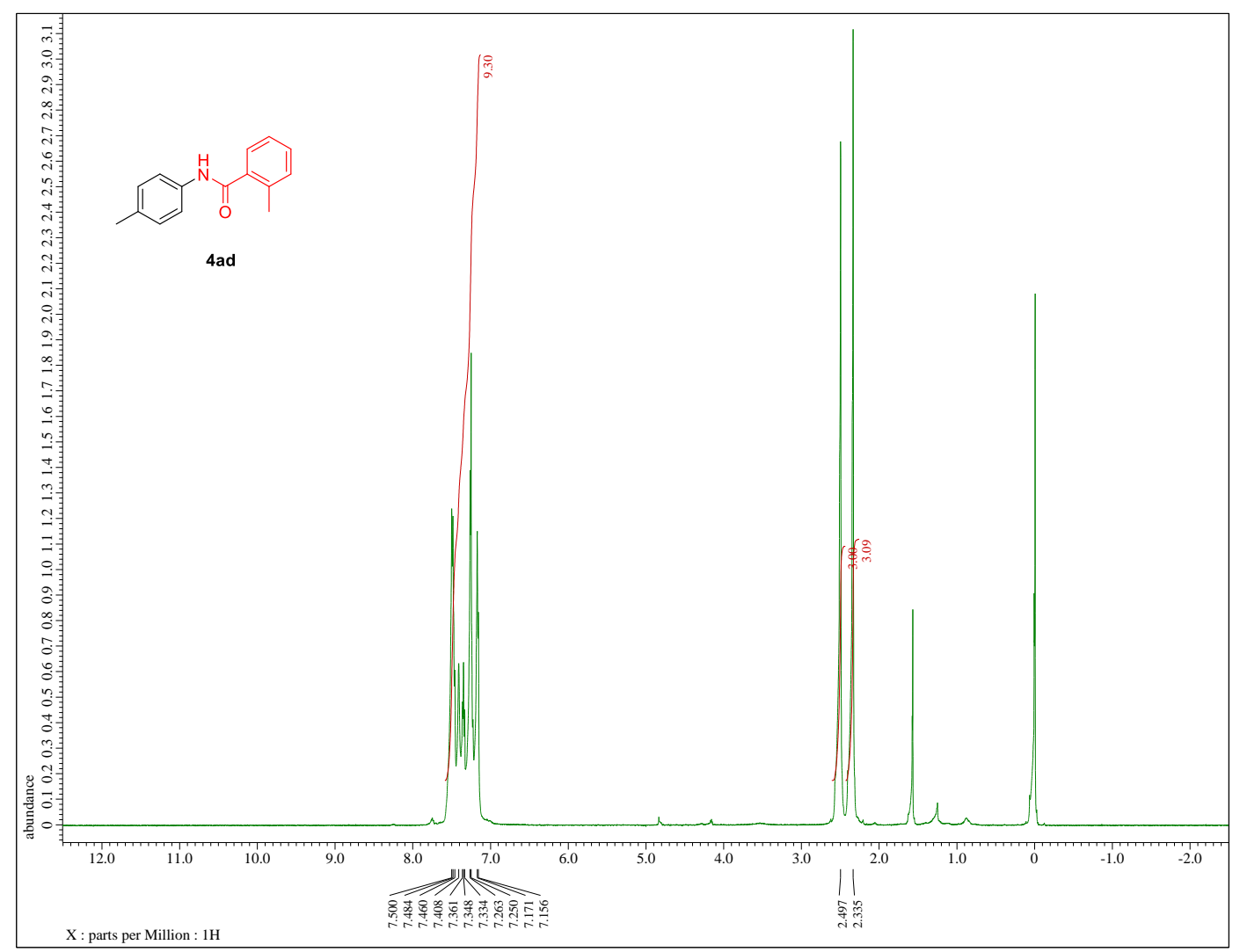

Figure S33-1. ${ }^{1} \mathrm{H}$ NMR spectrum of compound $4 \mathbf{a d}\left(500 \mathrm{MHz}, \mathrm{CDCl}_{3}\right)$

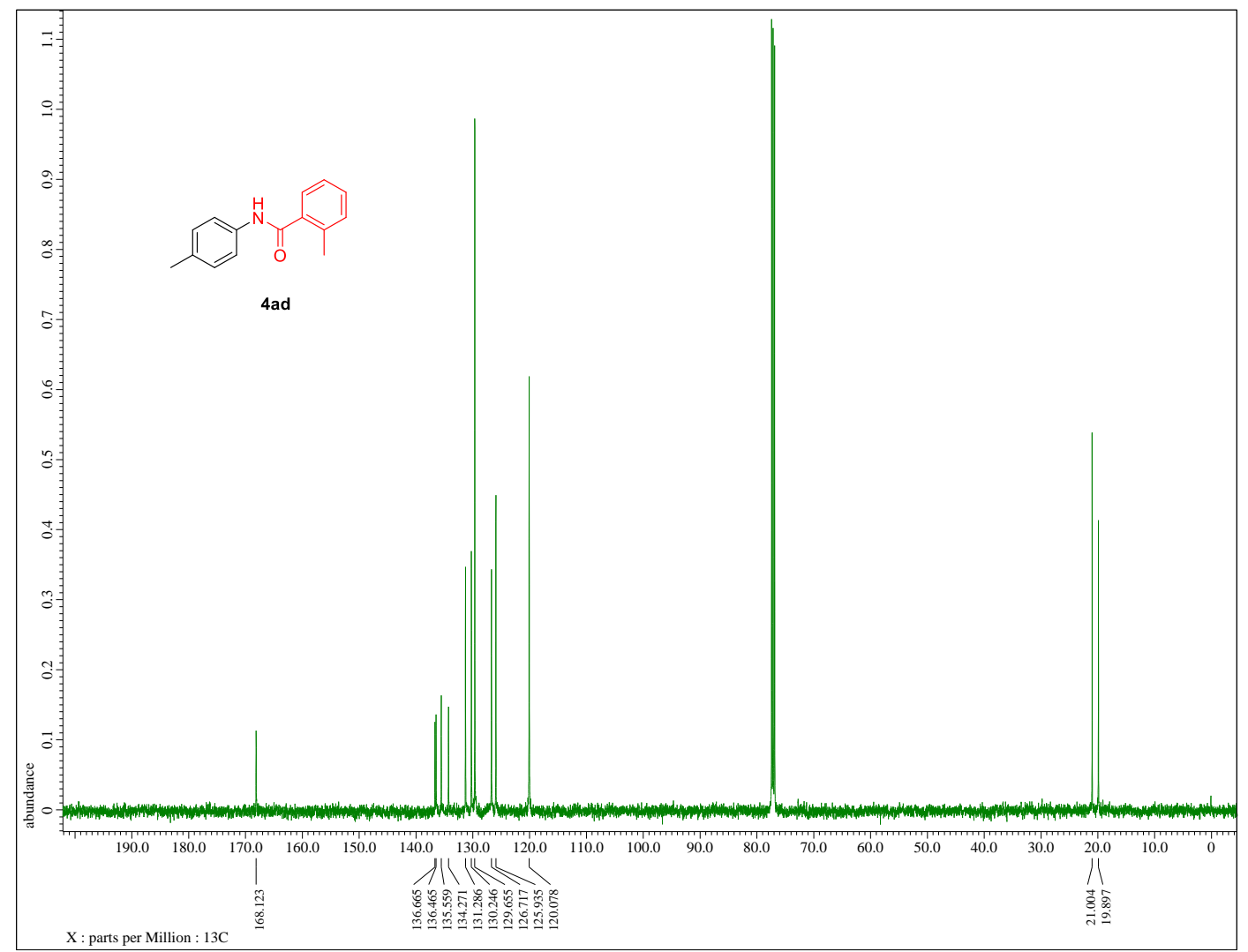

Figure S33-2. ${ }^{13} \mathrm{C}$ NMR spectrum of compound 4ad (125 MHz, $\left.\mathrm{CDCl}_{3}\right)$ 


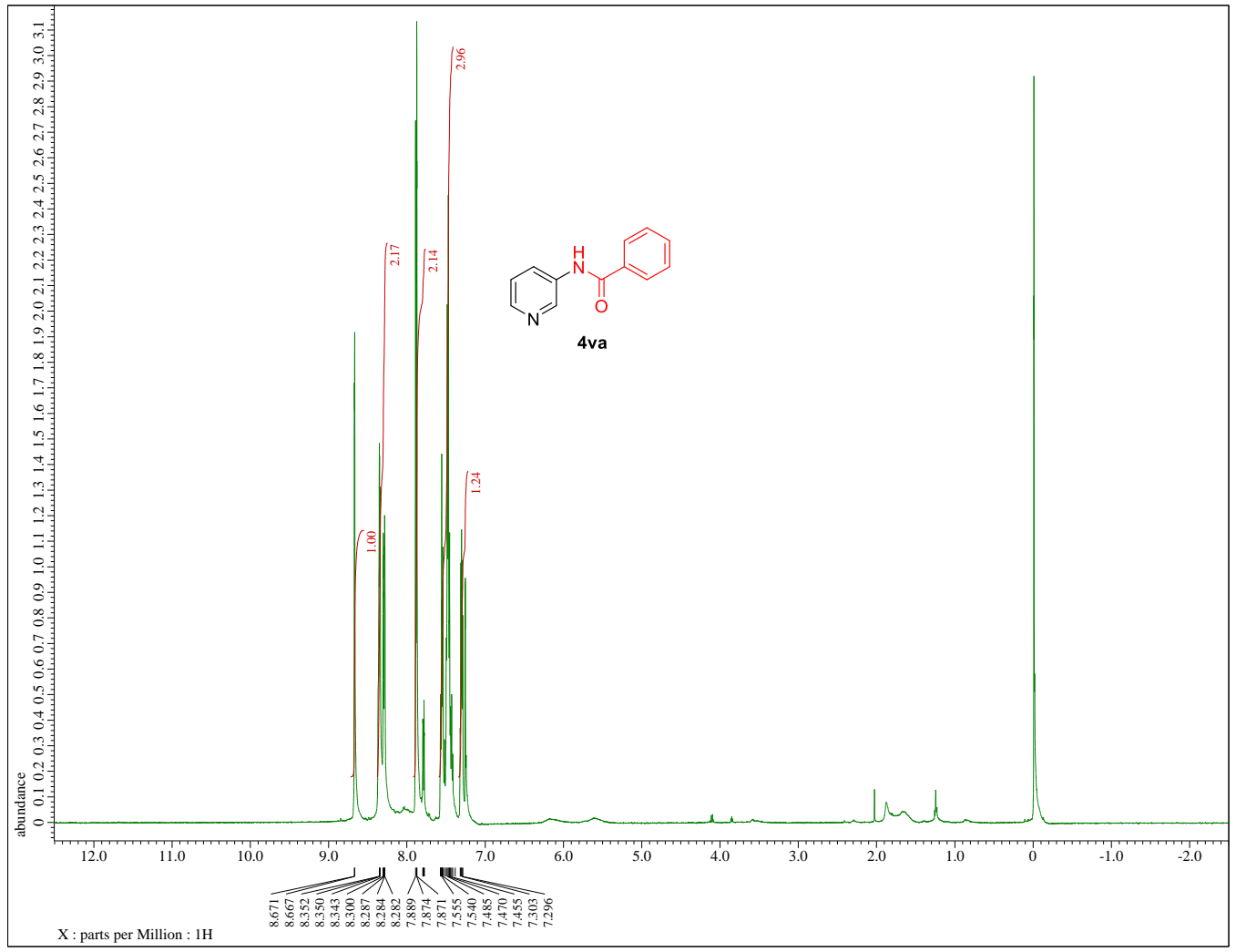

Figure S34-1. ${ }^{1} \mathrm{H}$ NMR spectrum of compound $4 \mathbf{v a}\left(500 \mathrm{MHz}, \mathrm{CDCl}_{3}\right)$

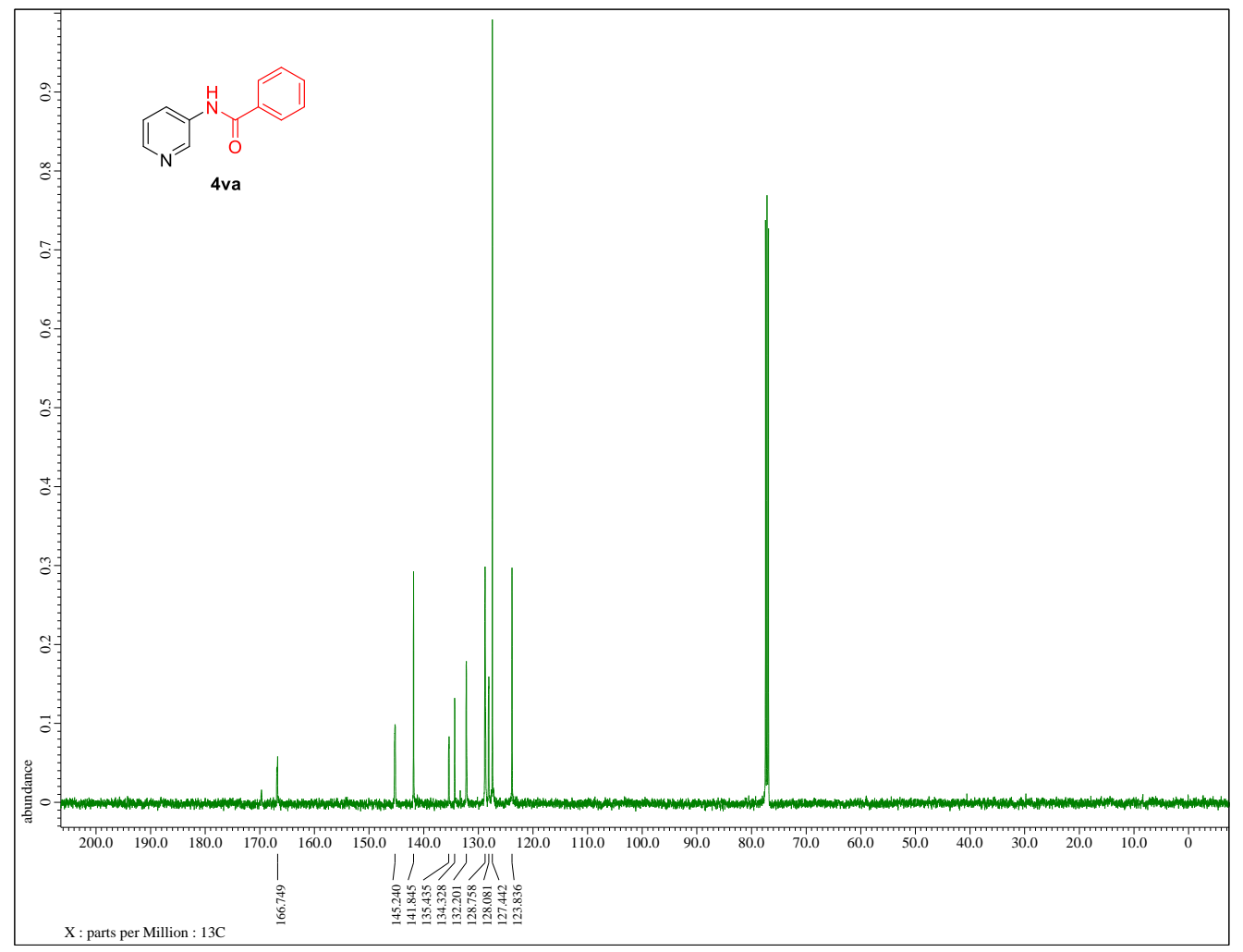

Figure S34-2. ${ }^{13} \mathrm{C}$ NMR spectrum of compound 4 va $\left(125 \mathrm{MHz}, \mathrm{CDCl}_{3}\right)$ 


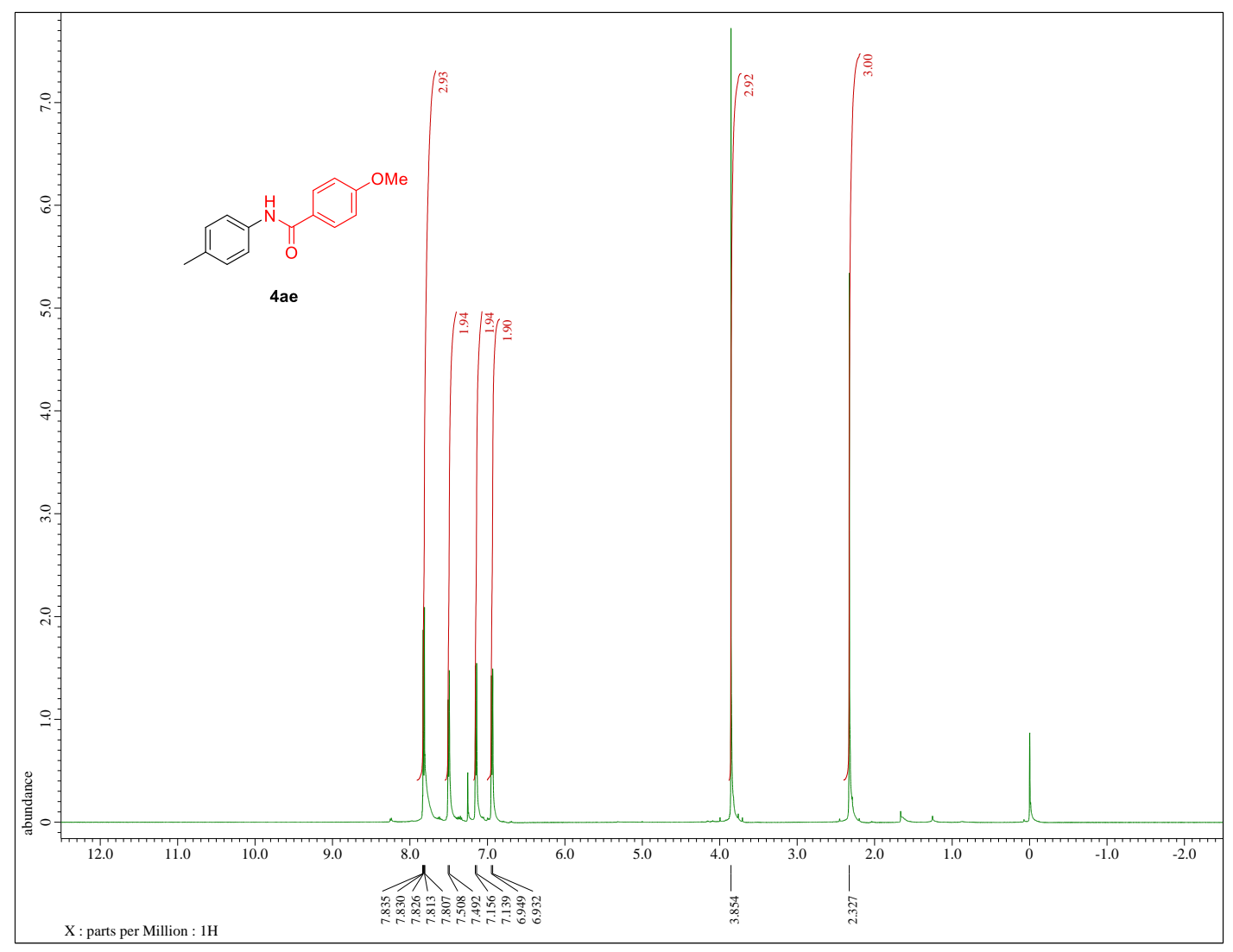

Figure S35-1. ${ }^{1} \mathrm{H}$ NMR spectrum of compound 4 ae $\left(500 \mathrm{MHz}, \mathrm{CDCl}_{3}\right)$

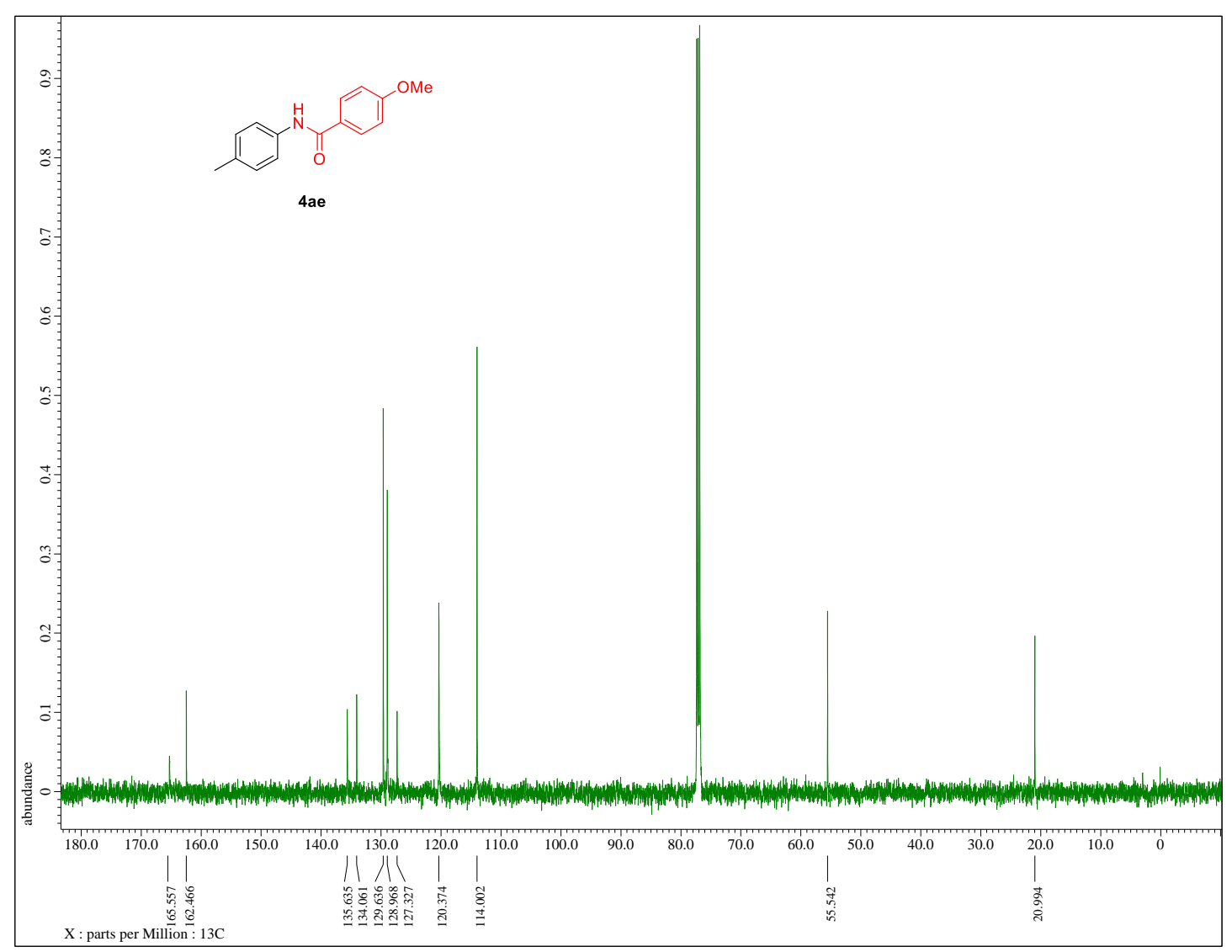

Figure S35-2. ${ }^{13} \mathrm{C}$ NMR spectrum of compound 4ae $\left(125 \mathrm{MHz}, \mathrm{CDCl}_{3}\right)$ 


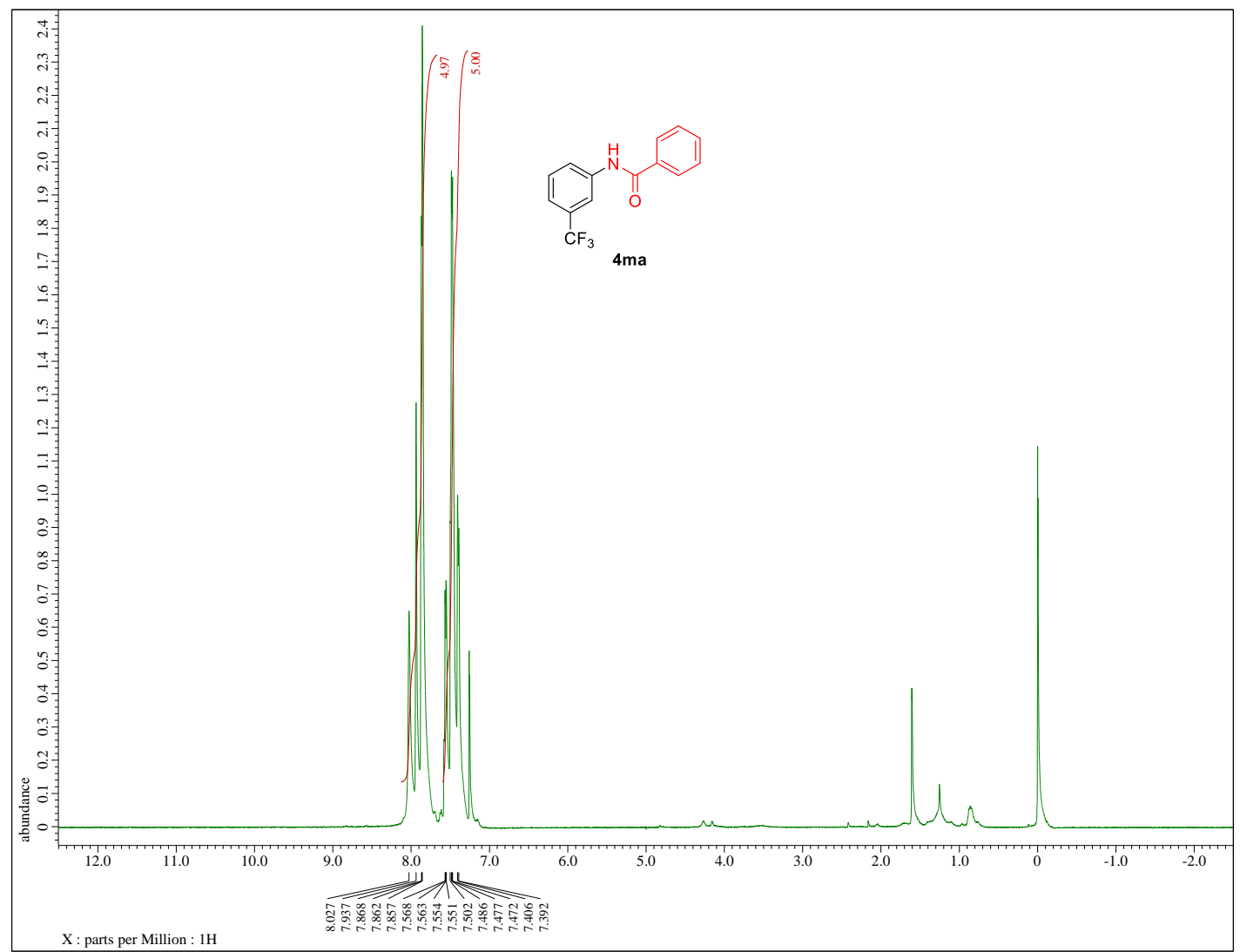

Figure S36-1. ${ }^{1} \mathrm{H}$ NMR spectrum of compound $4 \mathbf{m a}\left(500 \mathrm{MHz}, \mathrm{CDCl}_{3}\right)$

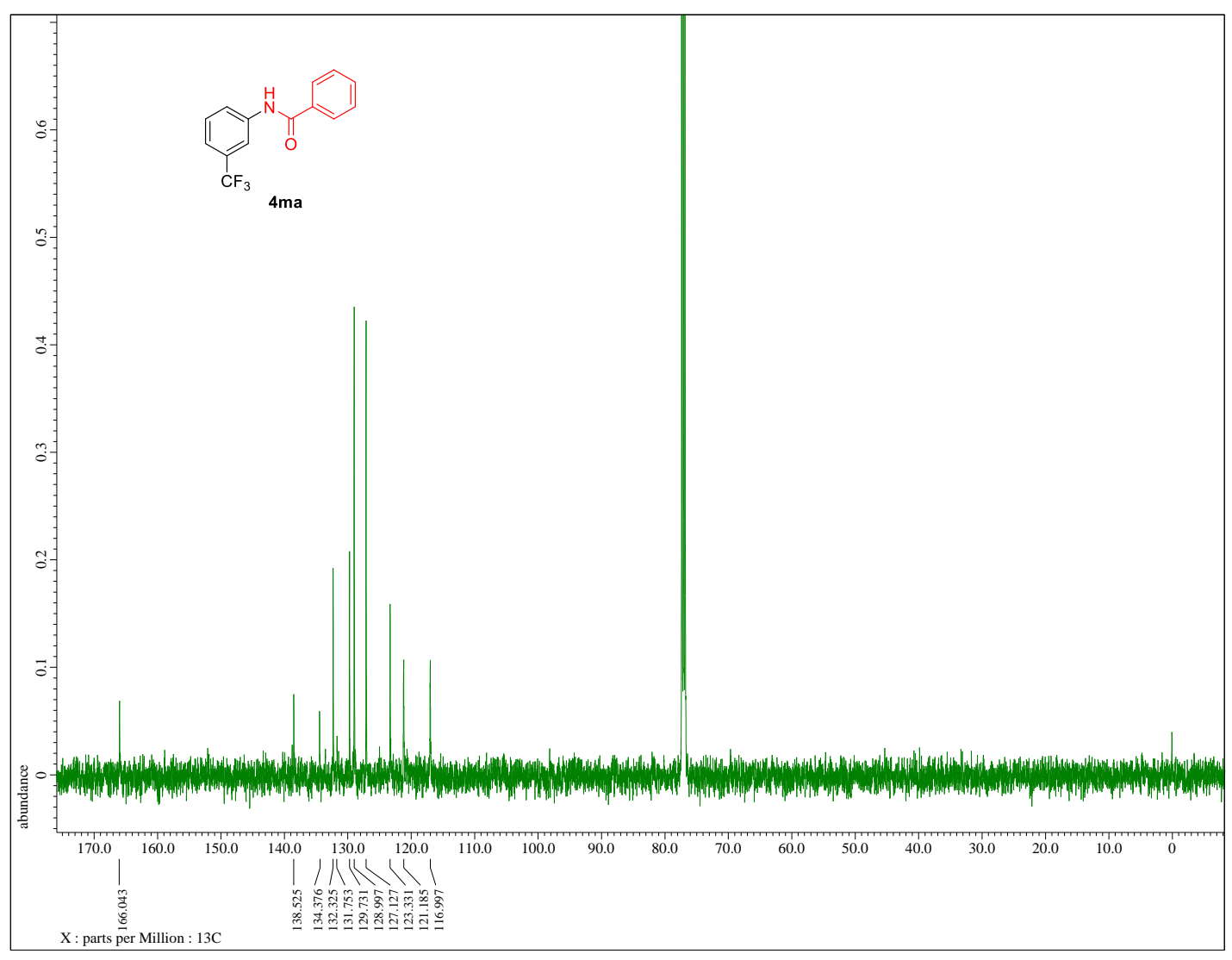

Figure S36-2. ${ }^{13} \mathrm{C}$ NMR spectrum of compound 4 ma (125 MHz, $\left.\mathrm{CDCl}_{3}\right)$ 


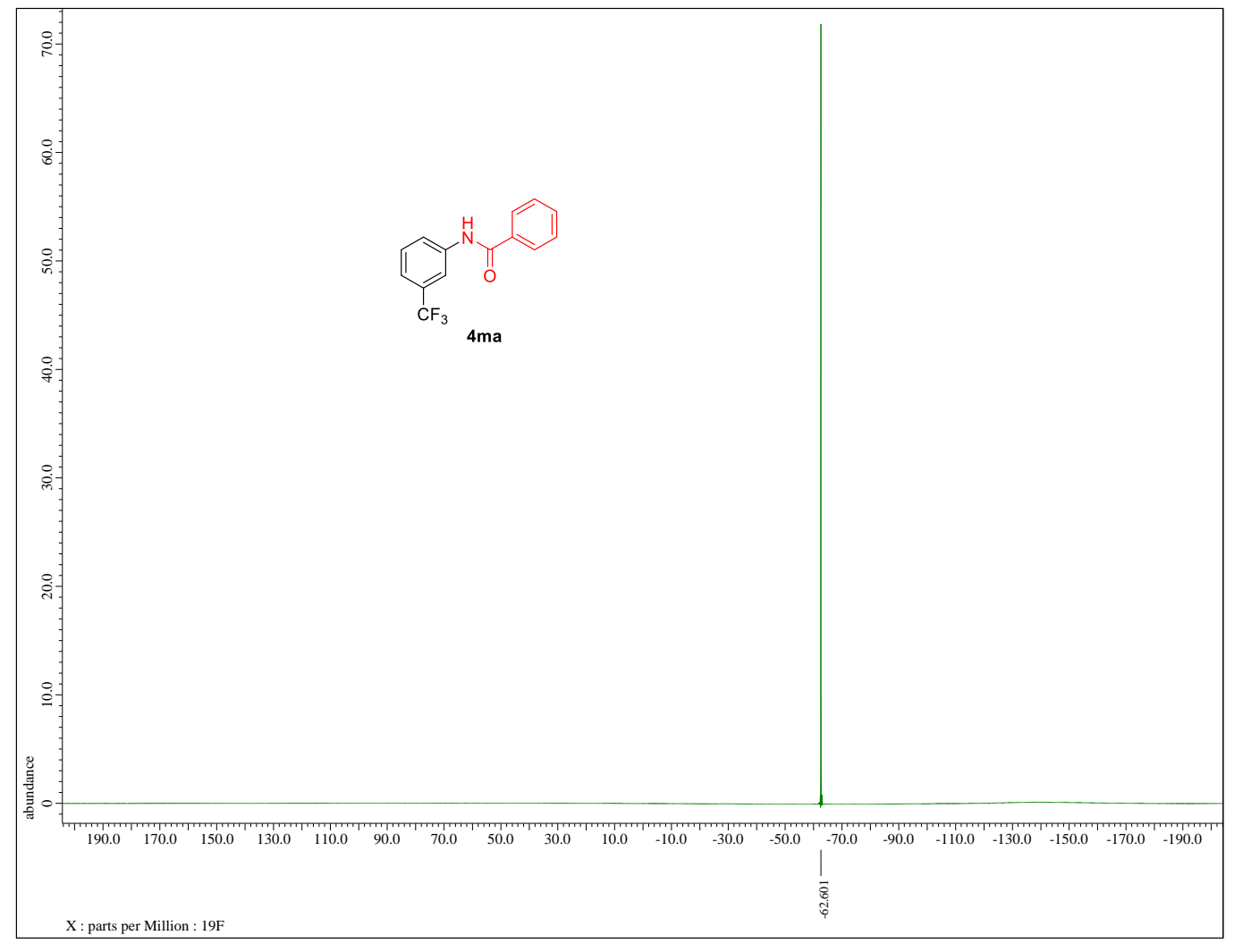

Figure S36-3. ${ }^{19} \mathrm{~F}$ NMR spectrum of compound $4 \mathrm{ma}\left(470 \mathrm{MHz}, \mathrm{CDCl}_{3}\right)$ 


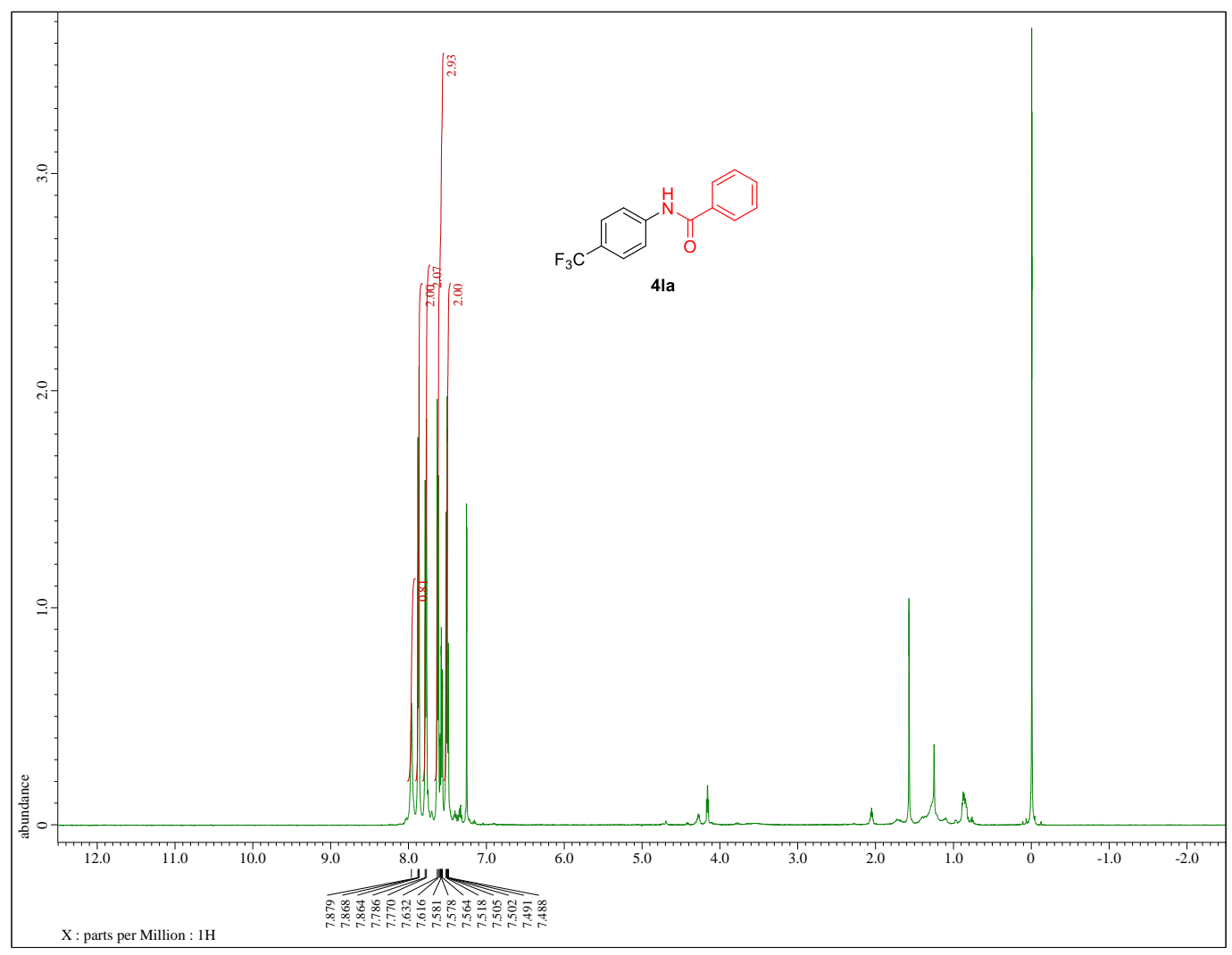

Figure S37-1. ${ }^{1} \mathrm{H}$ NMR spectrum of compound 4 la $\left(500 \mathrm{MHz}, \mathrm{CDCl}_{3}\right)$

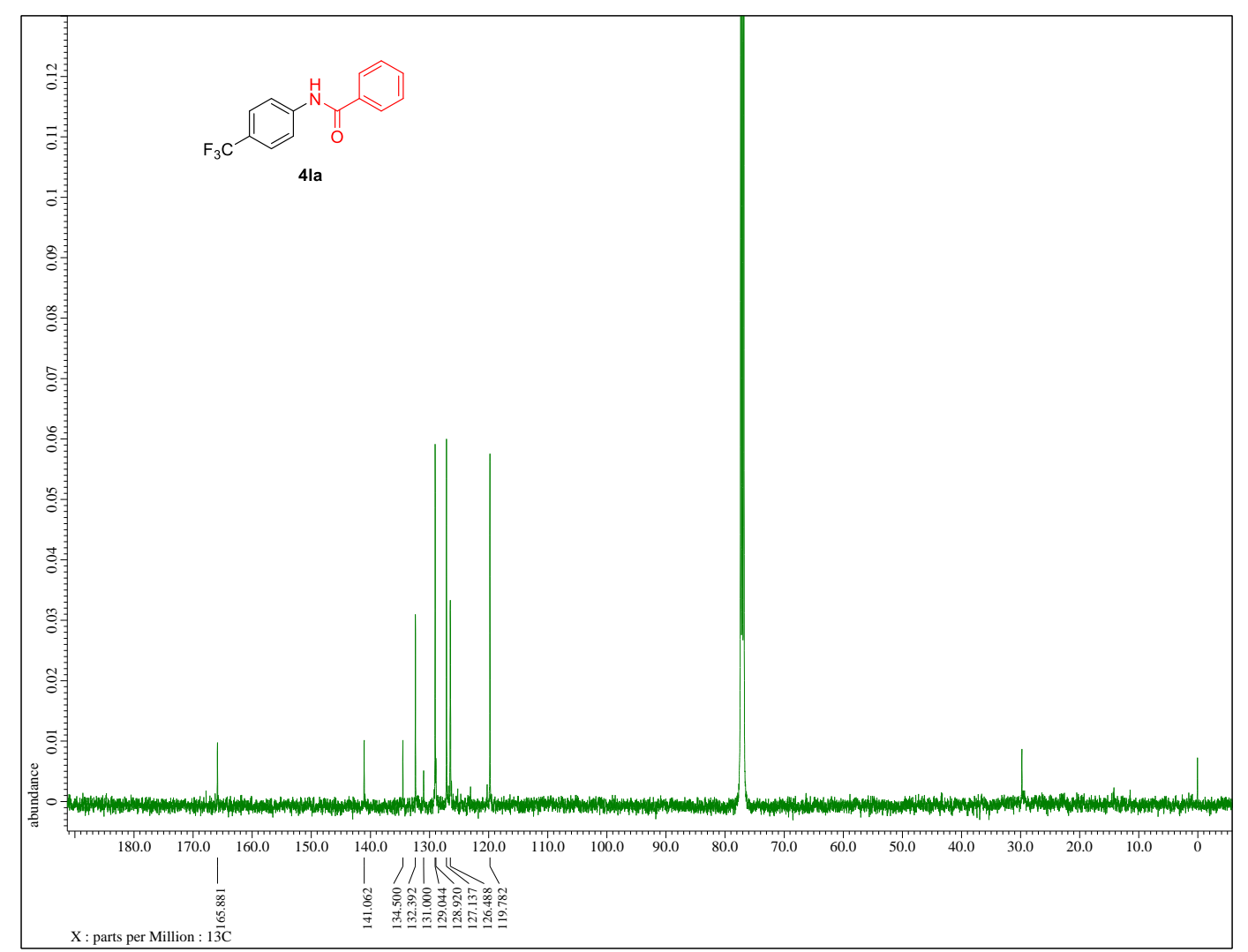

Figure S37-2. ${ }^{13} \mathrm{C}$ NMR spectrum of compound 4la $\left(125 \mathrm{MHz}, \mathrm{CDCl}_{3}\right)$ 


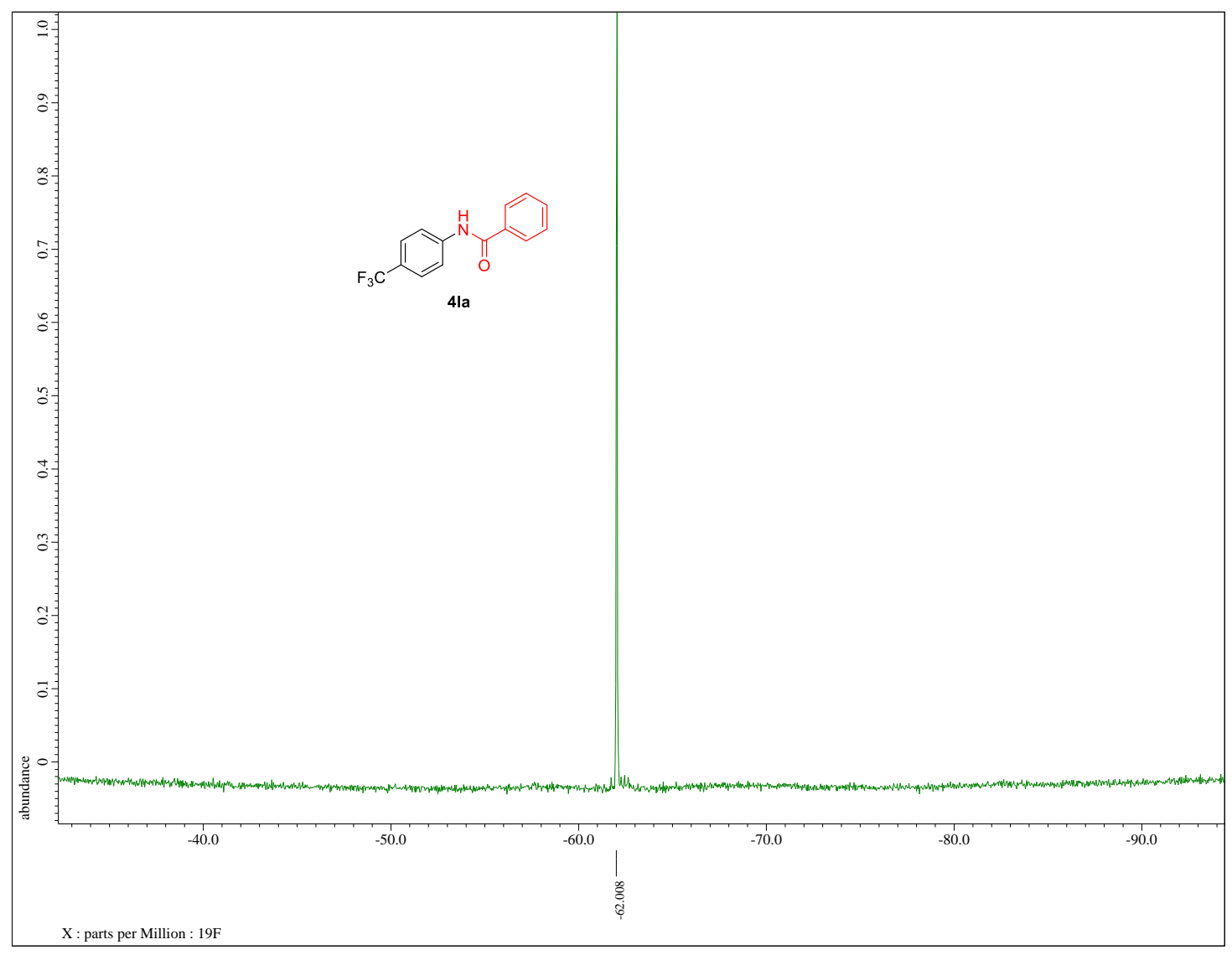

Figure S37-3. ${ }^{19} \mathrm{~F}$ NMR spectrum of compound $4 \mathrm{la}\left(470 \mathrm{MHz}, \mathrm{CDCl}_{3}\right)$ 


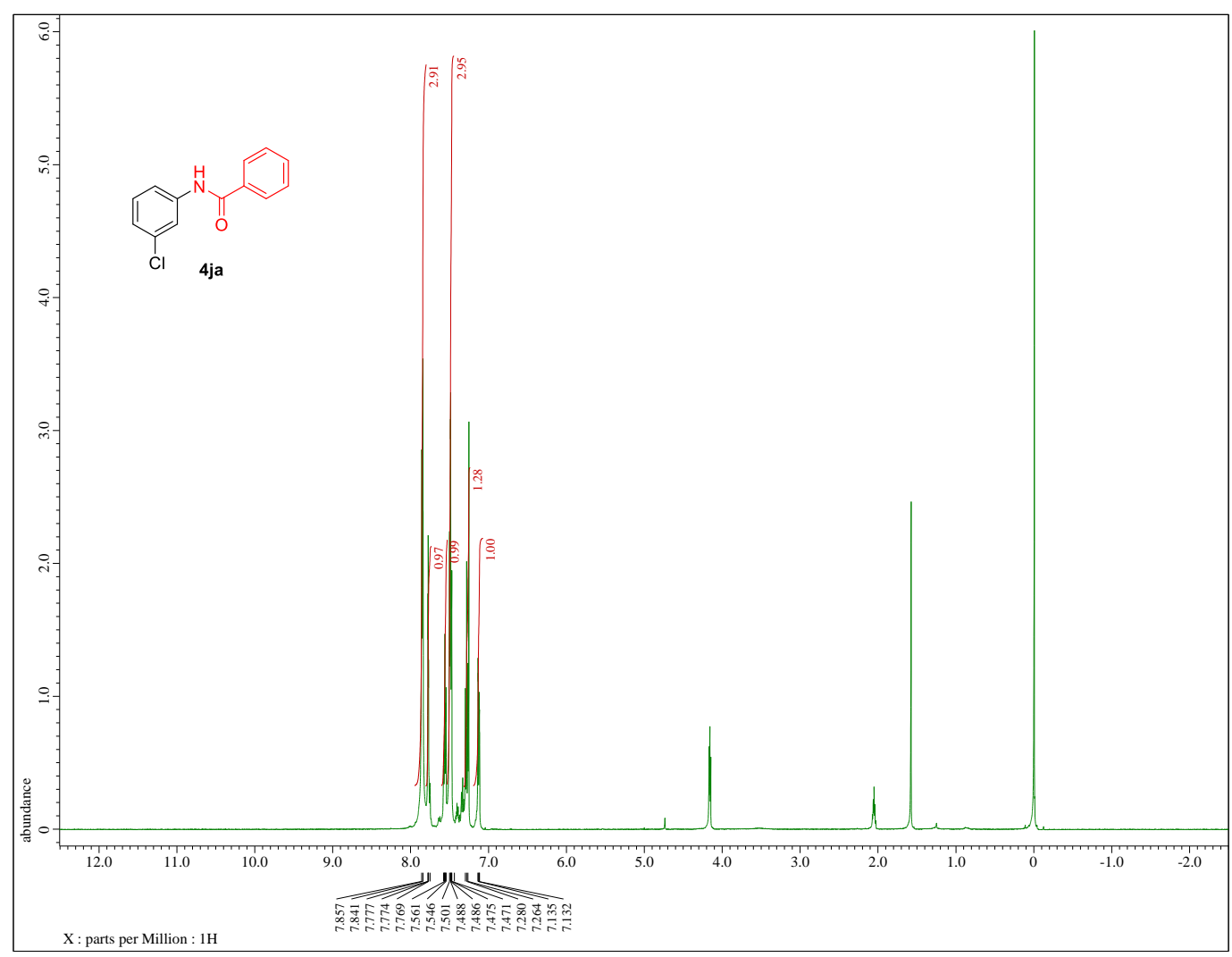

Figure S38-1. ${ }^{1} \mathrm{H}$ NMR spectrum of compound $\mathbf{4 j a}\left(500 \mathrm{MHz}, \mathrm{CDCl}_{3}\right)$

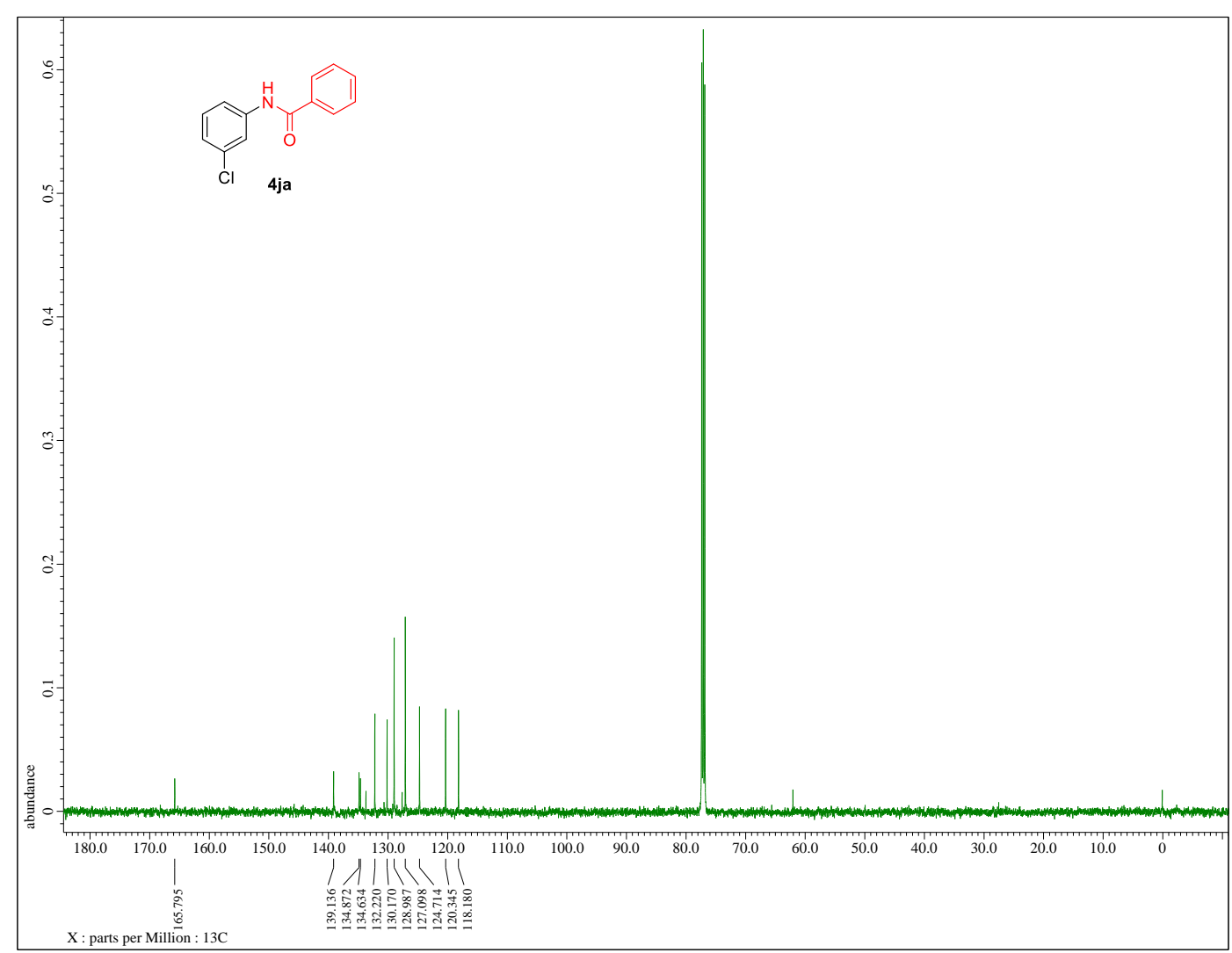

Figure S38-2. ${ }^{13} \mathrm{C}$ NMR spectrum of compound $\mathbf{4 j a}\left(125 \mathrm{MHz}, \mathrm{CDCl}_{3}\right)$ 


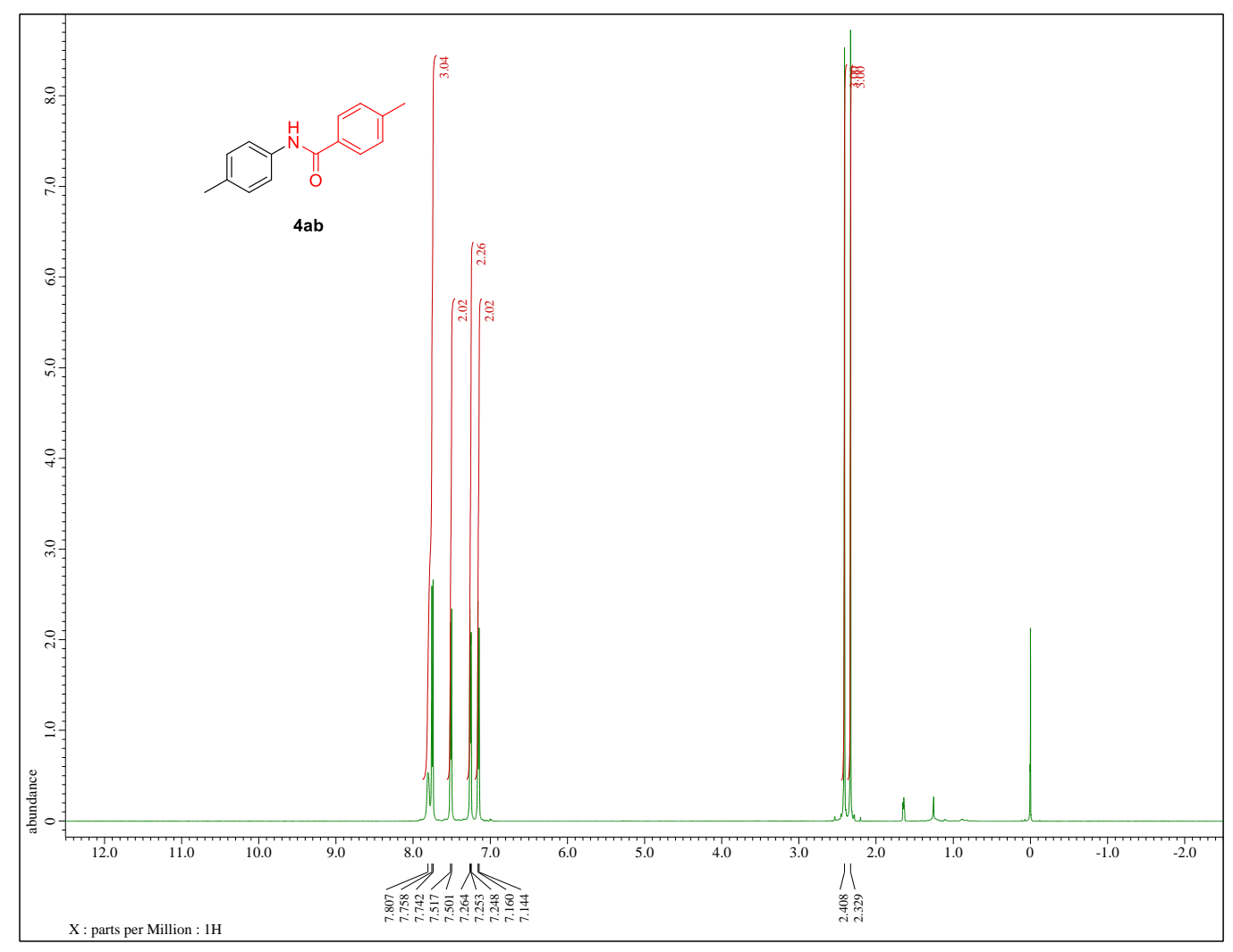

Figure S39-1. ${ }^{1} \mathrm{H}$ NMR spectrum of compound $4 \mathbf{a b}\left(500 \mathrm{MHz}, \mathrm{CDCl}_{3}\right)$

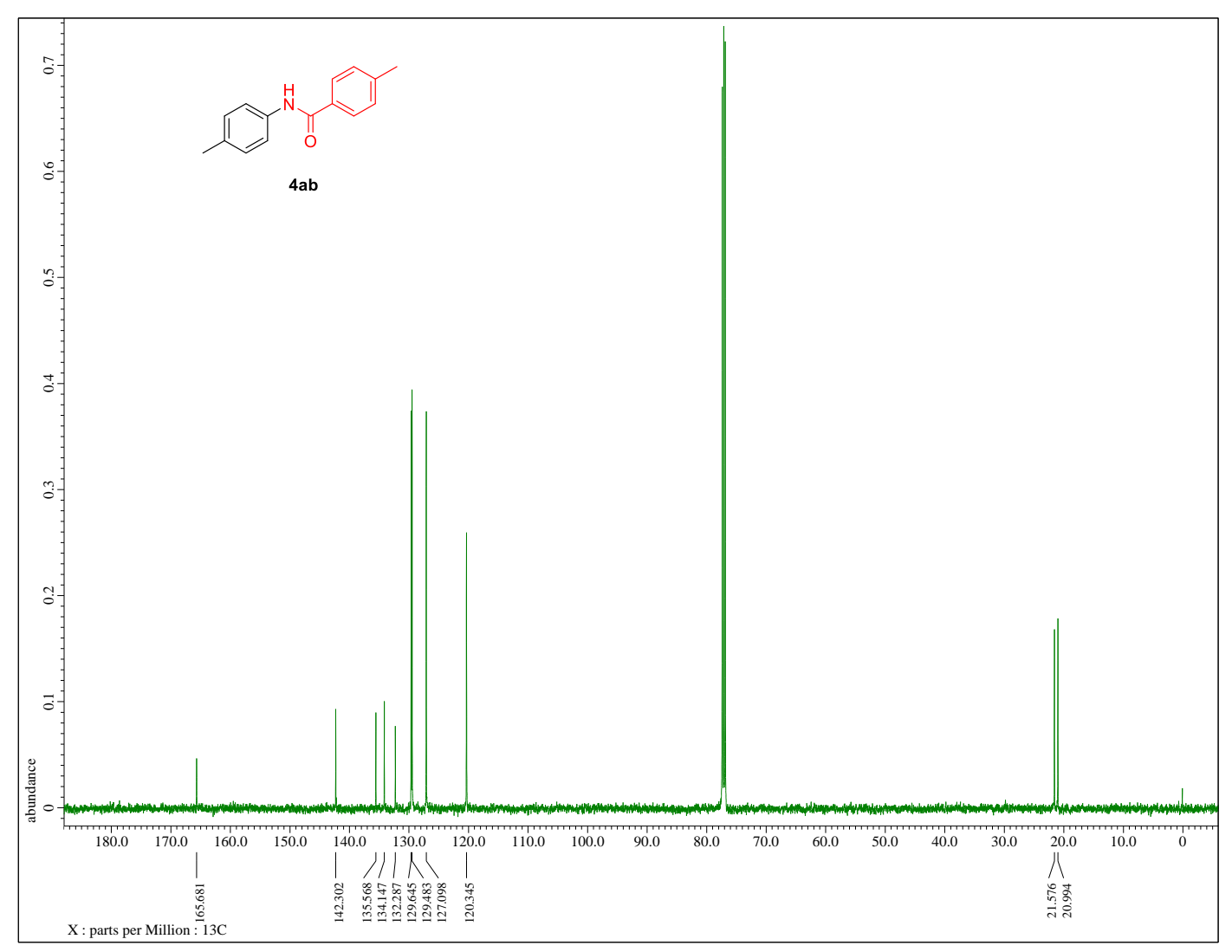

Figure S39-2. ${ }^{13} \mathrm{C}$ NMR spectrum of compound $4 \mathbf{a b}\left(125 \mathrm{MHz}, \mathrm{CDCl}_{3}\right)$ 


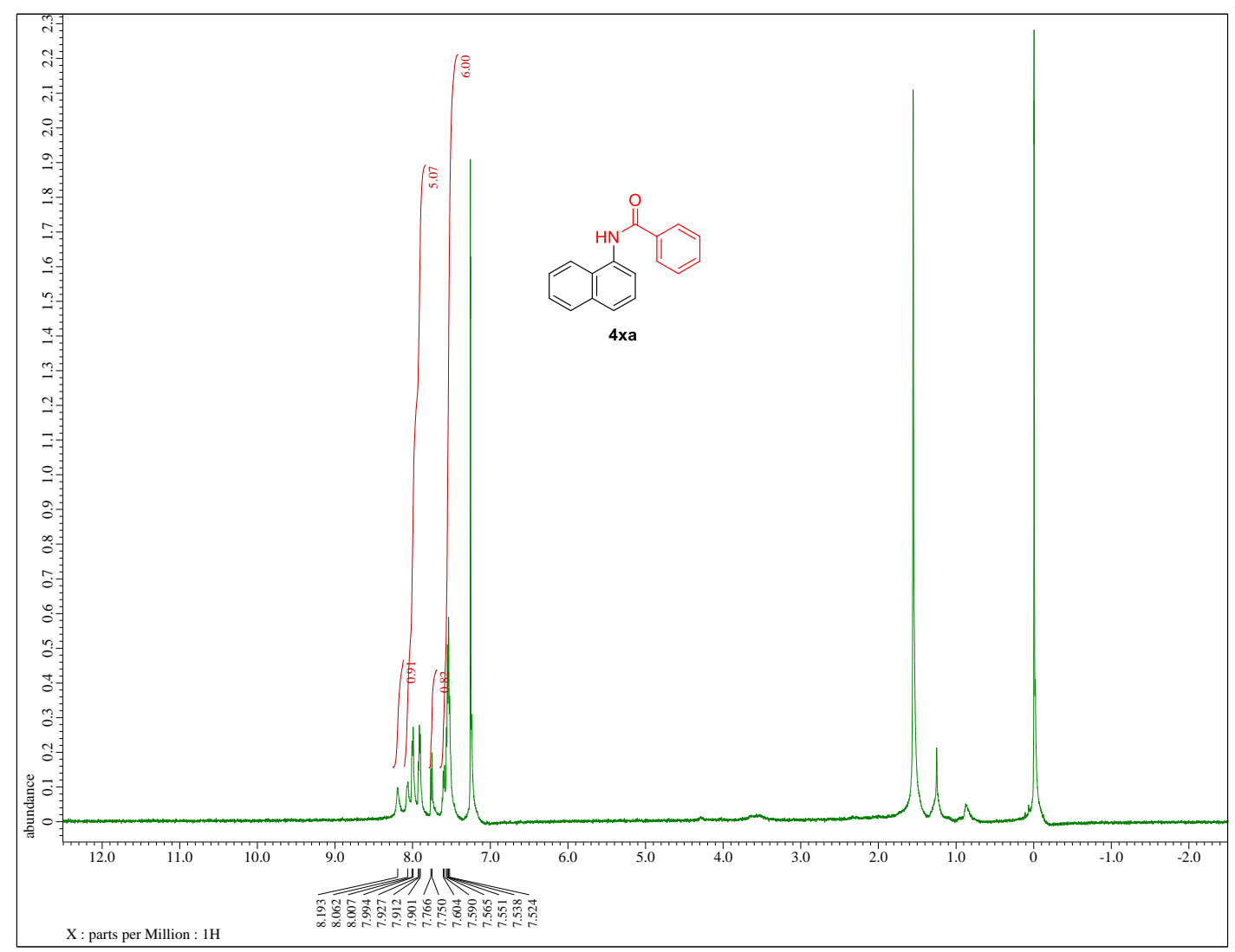

Figure S40-1. ${ }^{1} \mathrm{H}$ NMR spectrum of compound 4 xa $\left(500 \mathrm{MHz}, \mathrm{CDCl}_{3}\right)$

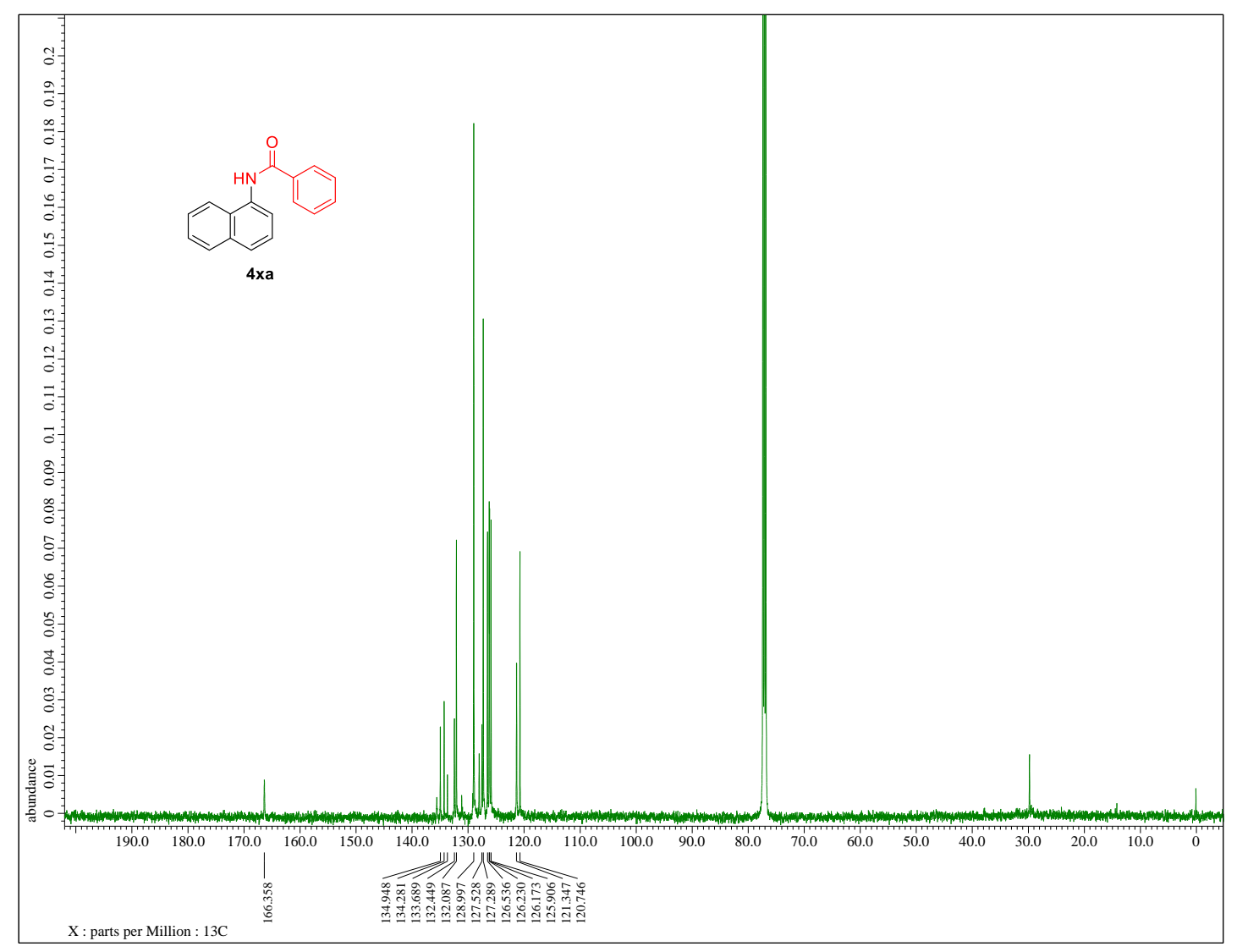

Figure S40-2. ${ }^{13} \mathrm{C}$ NMR spectrum of compound $4 \times \mathbf{x a}\left(125 \mathrm{MHz}, \mathrm{CDCl}_{3}\right)$ 


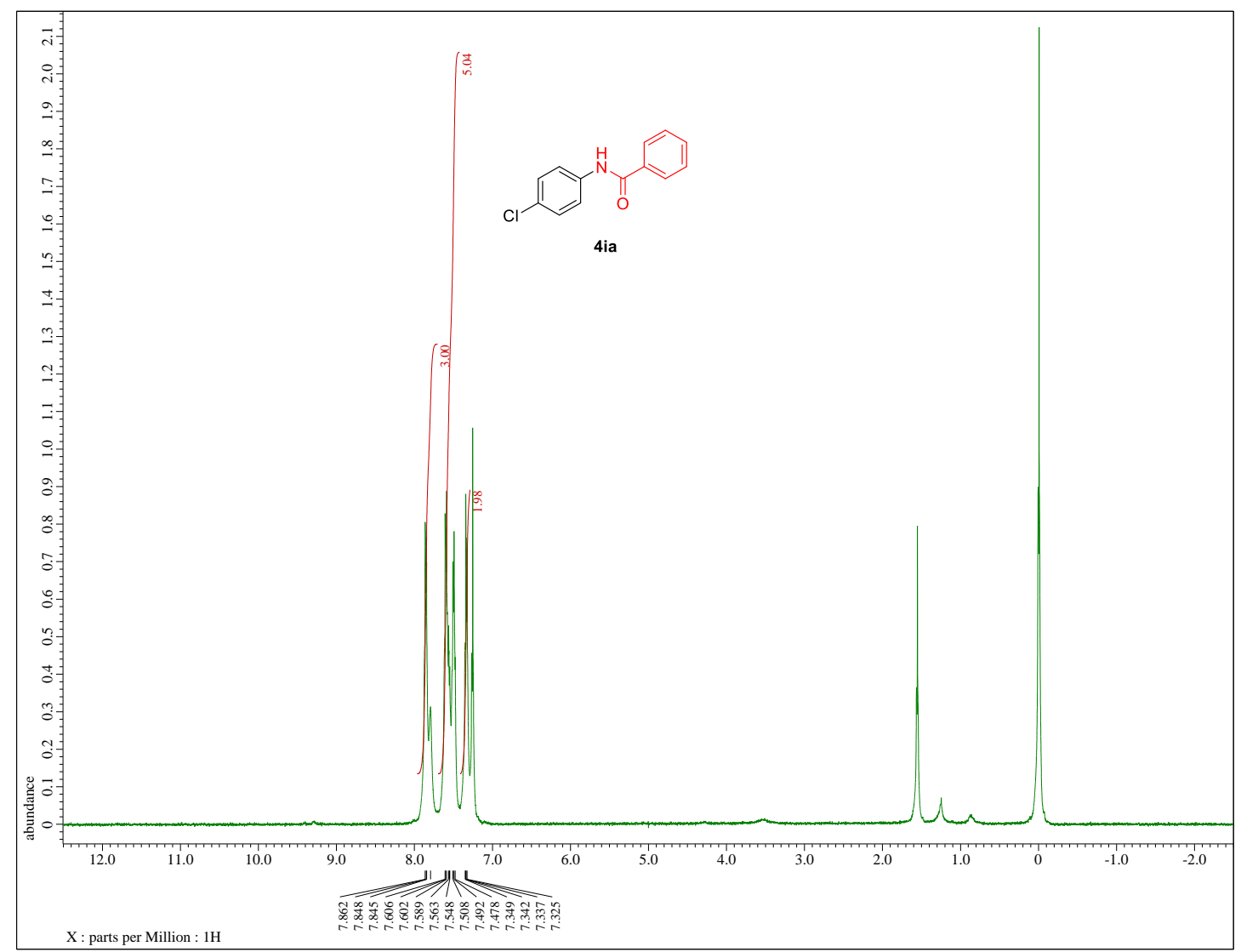

Figure S41-1. ${ }^{1} \mathrm{H}$ NMR spectrum of compound 4 ia $\left(500 \mathrm{MHz}, \mathrm{CDCl}_{3}\right)$

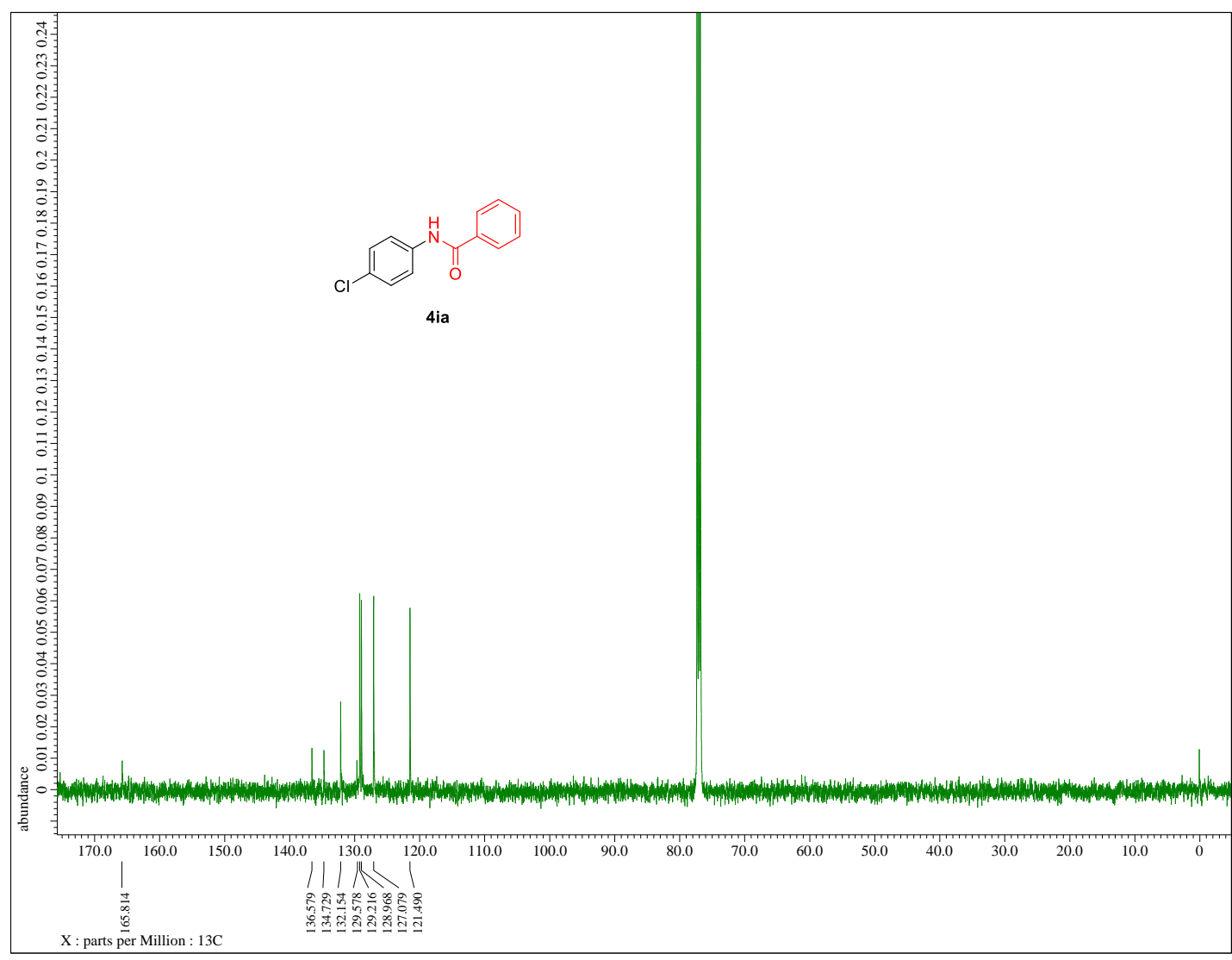

Figure S41-2. ${ }^{13} \mathrm{C}$ NMR spectrum of compound 4ia $\left(125 \mathrm{MHz}, \mathrm{CDCl}_{3}\right)$ 


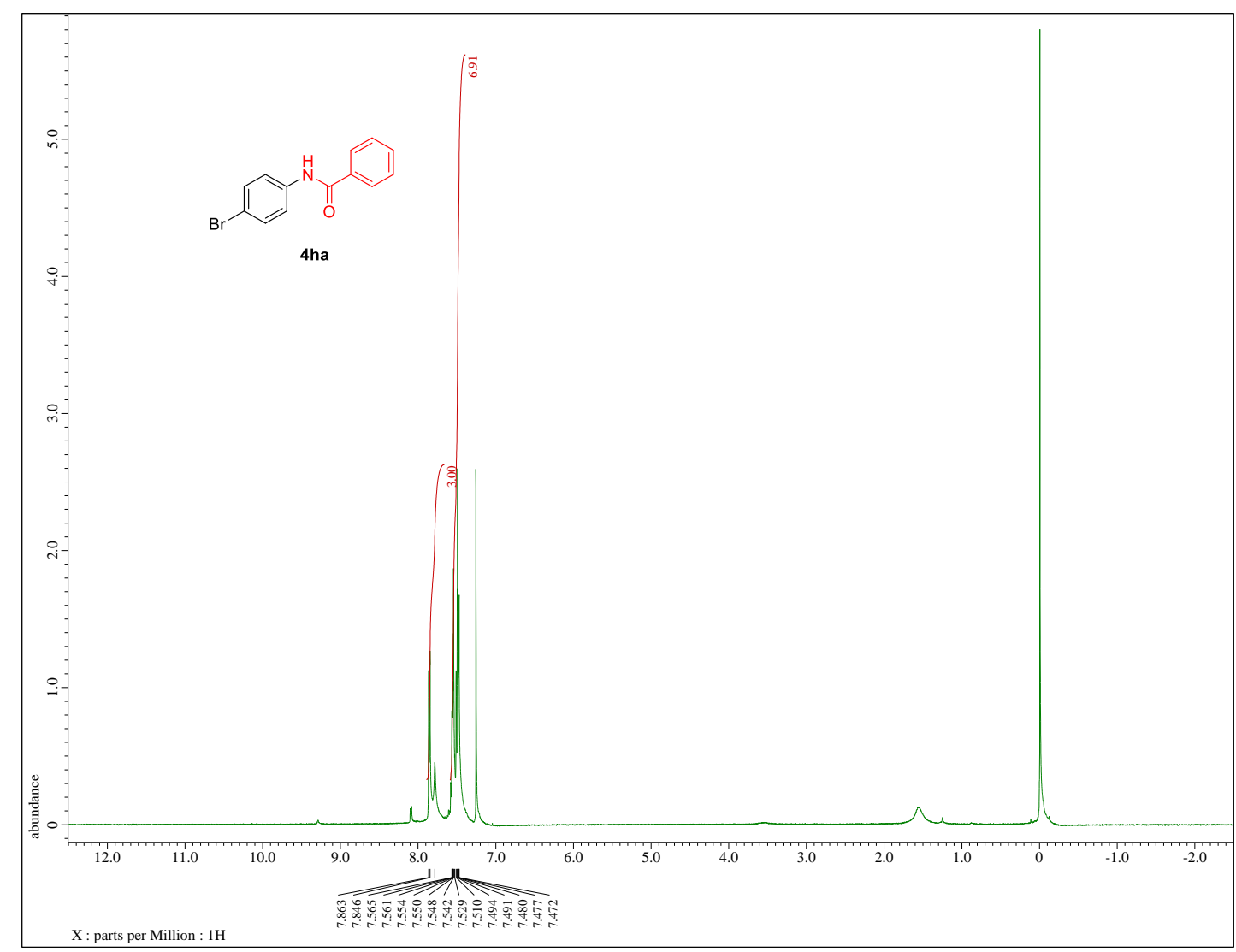

Figure S42-1. ${ }^{1} \mathrm{H}$ NMR spectrum of compound 4 ha $\left(500 \mathrm{MHz}, \mathrm{CDCl}_{3}\right)$

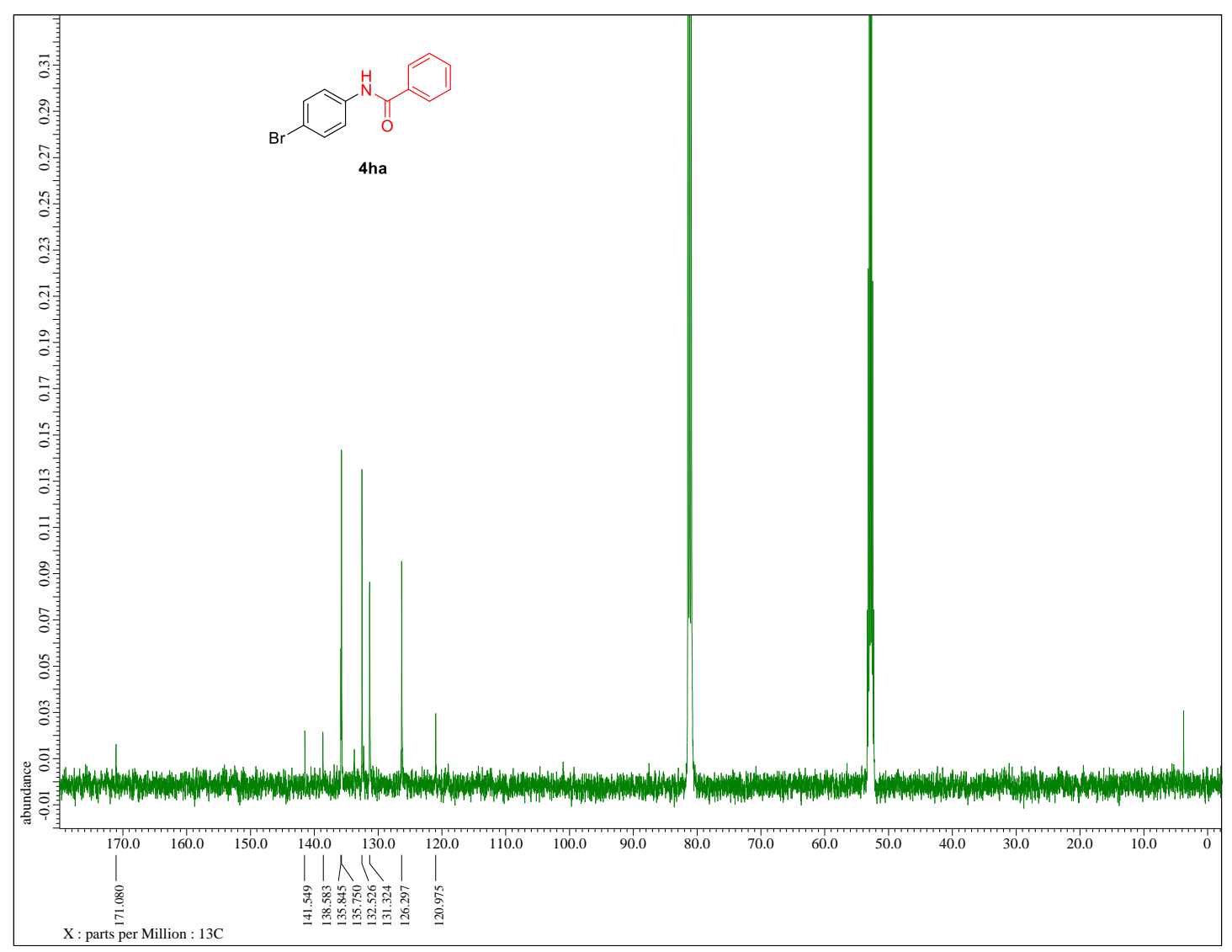

Figure S42-2. ${ }^{13} \mathrm{C}$ NMR spectrum of compound 4 ha $\left(125 \mathrm{MHz}, \mathrm{CDCl}_{3}\right.$ with 2 drops methanol- $\left.\mathrm{d}_{3}\right)$ 


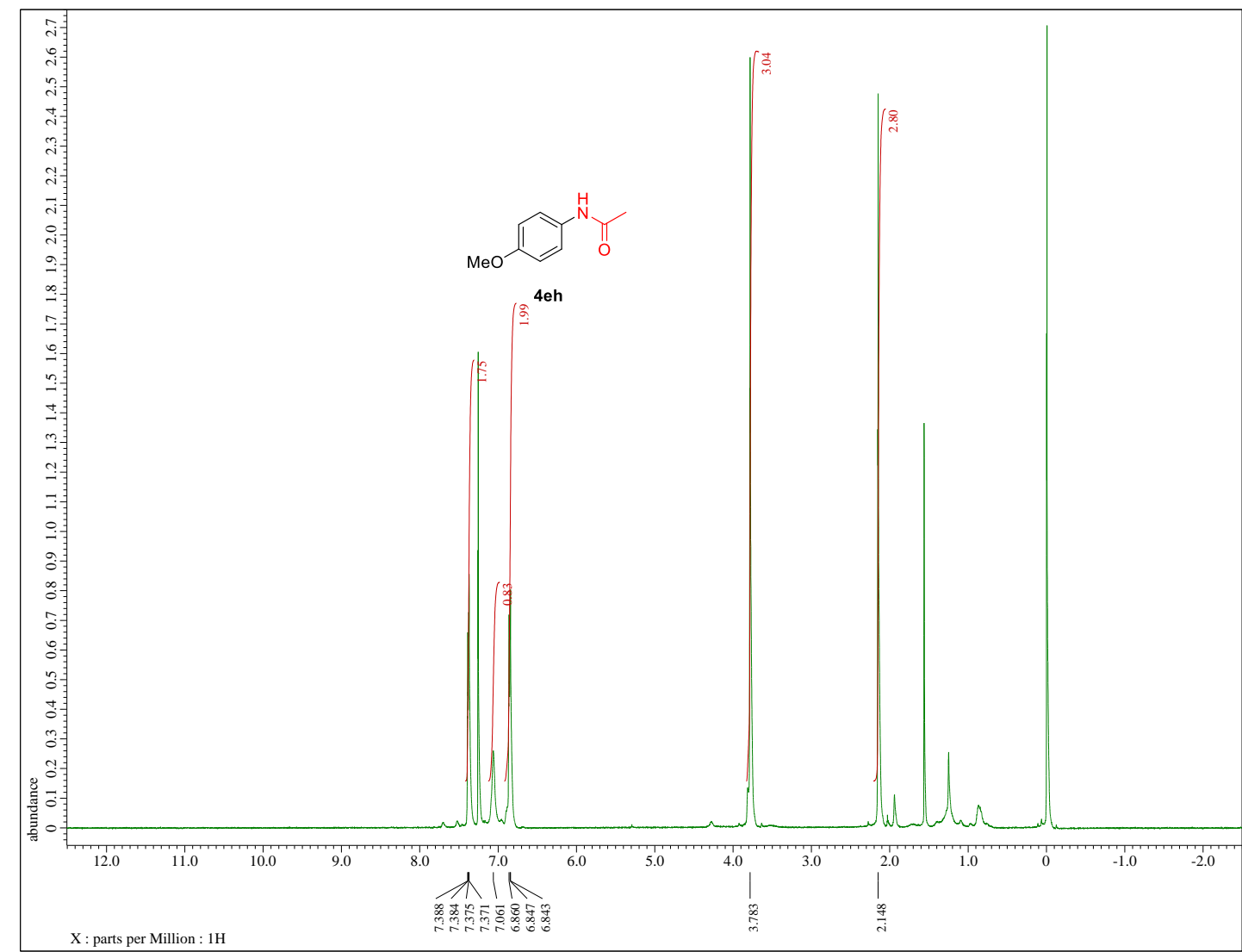

Figure S43-1. ${ }^{1} \mathrm{H}$ NMR spectrum of compound $4 \mathbf{e h}\left(500 \mathrm{MHz}, \mathrm{CDCl}_{3}\right)$

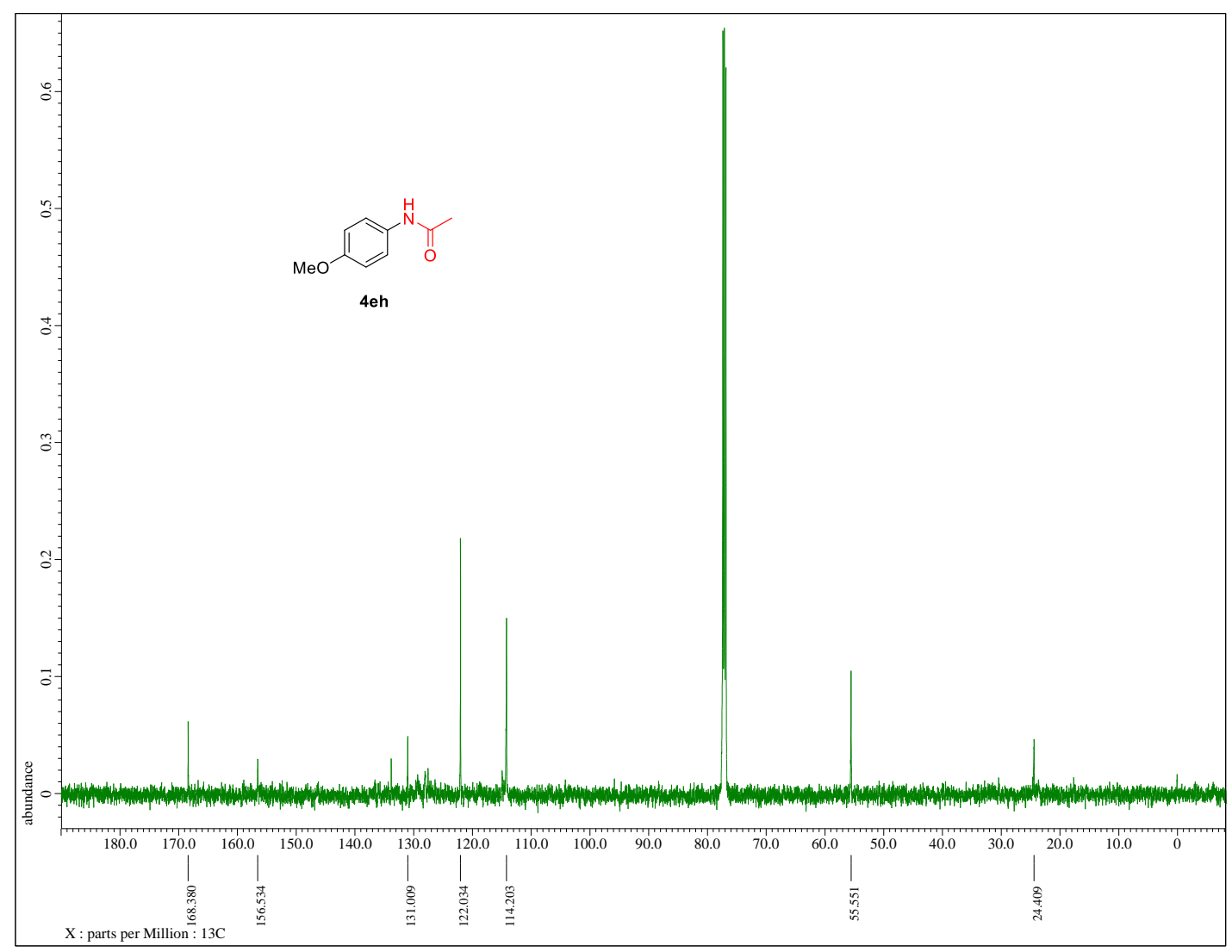

Figure S43-2. ${ }^{13} \mathrm{C}$ NMR spectrum of compound 4eh $\left(125 \mathrm{MHz}, \mathrm{CDCl}_{3}\right)$ 


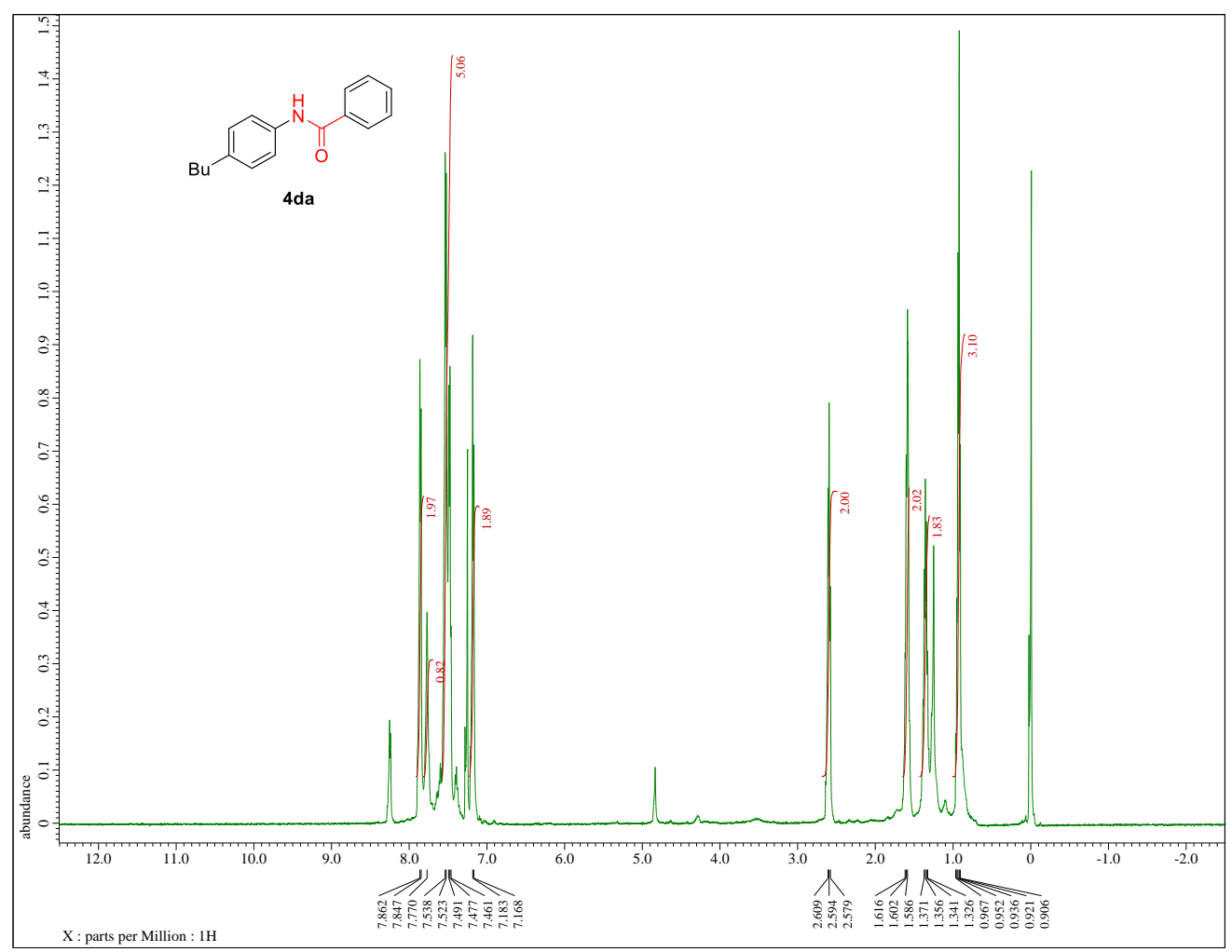

Figure S44-1. ${ }^{1} \mathrm{H}$ NMR spectrum of compound $4 \mathrm{da}\left(500 \mathrm{MHz}, \mathrm{CDCl}_{3}\right)$

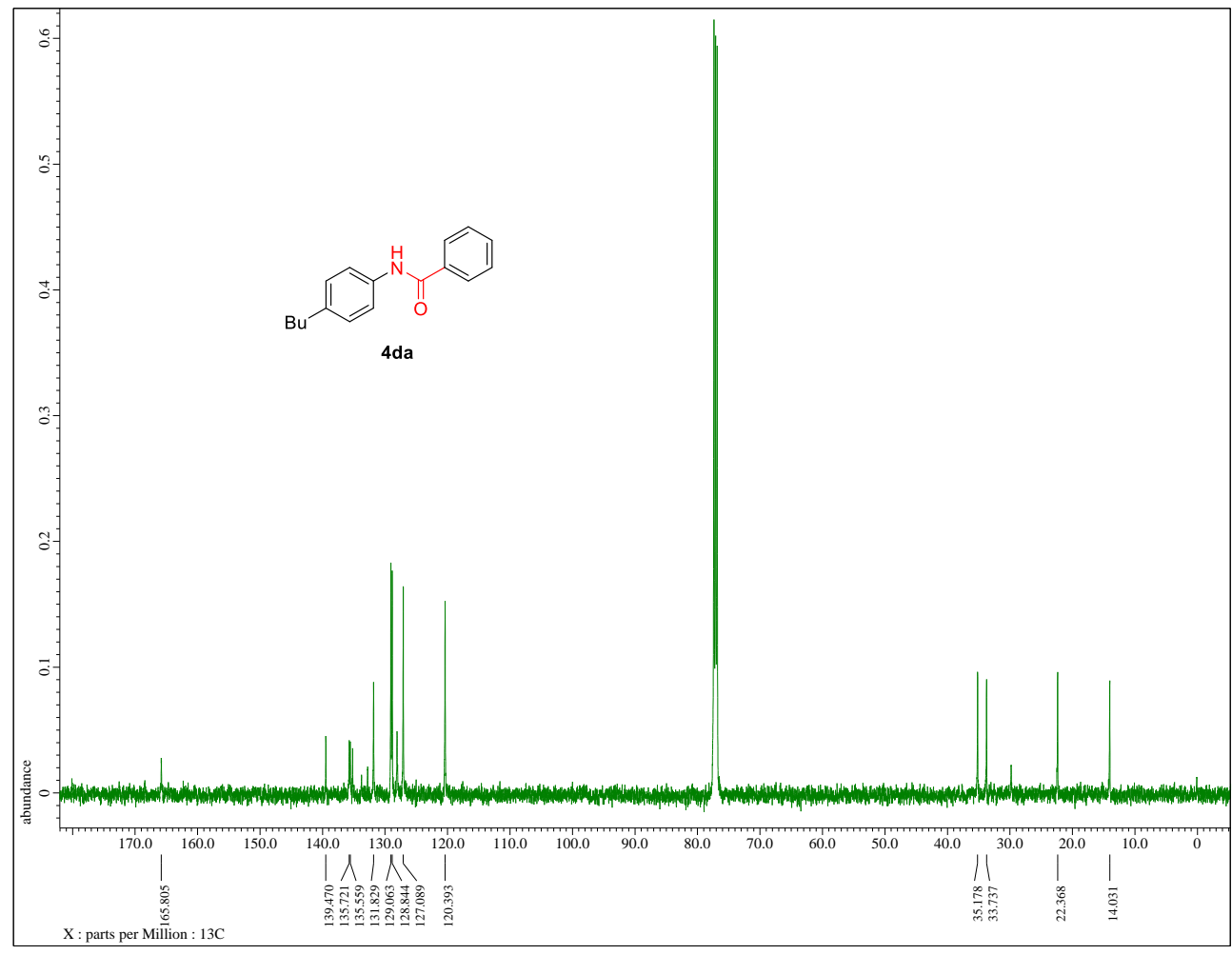

Figure S44-2. ${ }^{13} \mathrm{C}$ NMR spectrum of compound 4 da $\left(125 \mathrm{MHz}, \mathrm{CDCl}_{3}\right)$ 


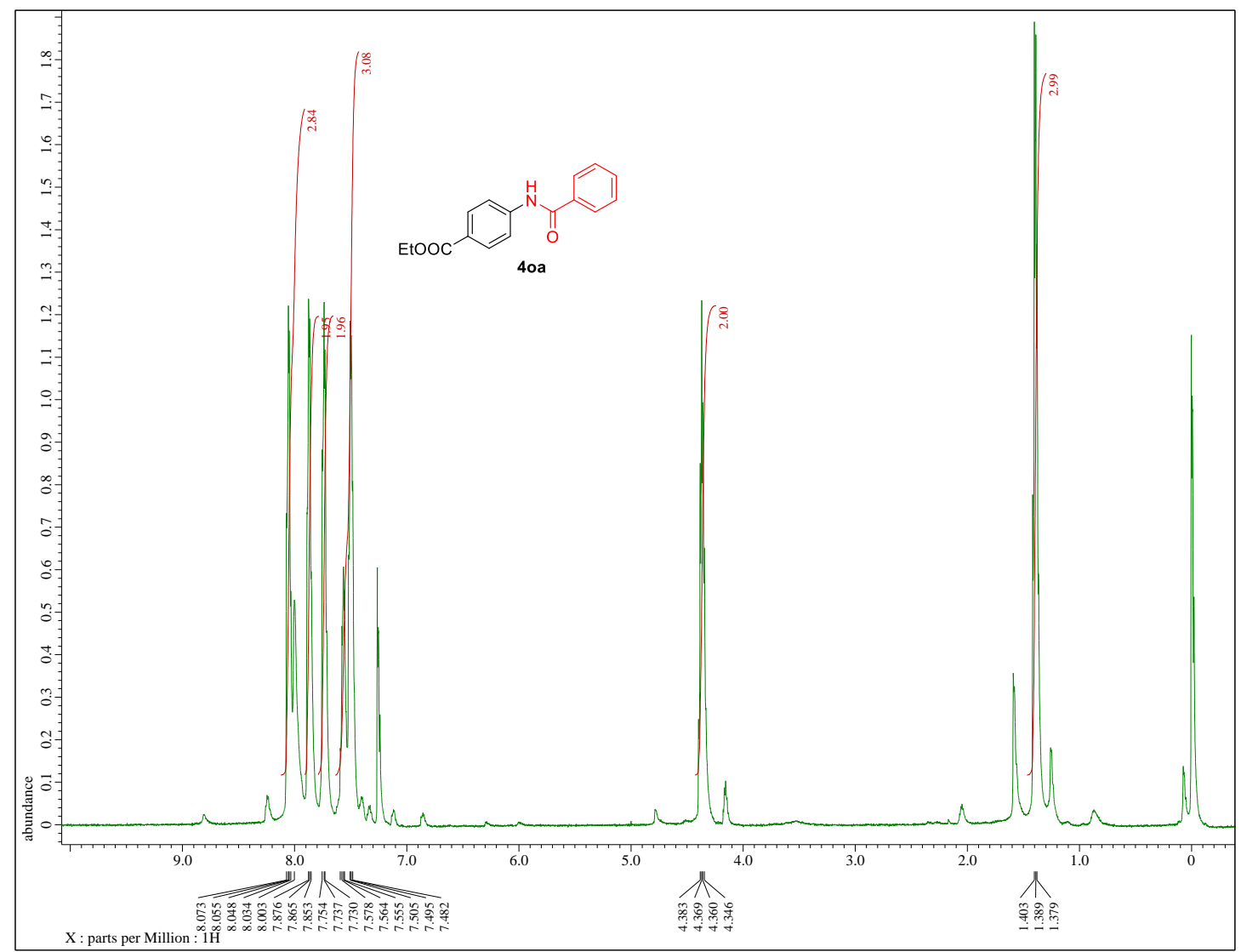

Figure S45-1. ${ }^{1} \mathrm{H}$ NMR spectrum of compound $40 a\left(500 \mathrm{MHz}, \mathrm{CDCl}_{3}\right)$

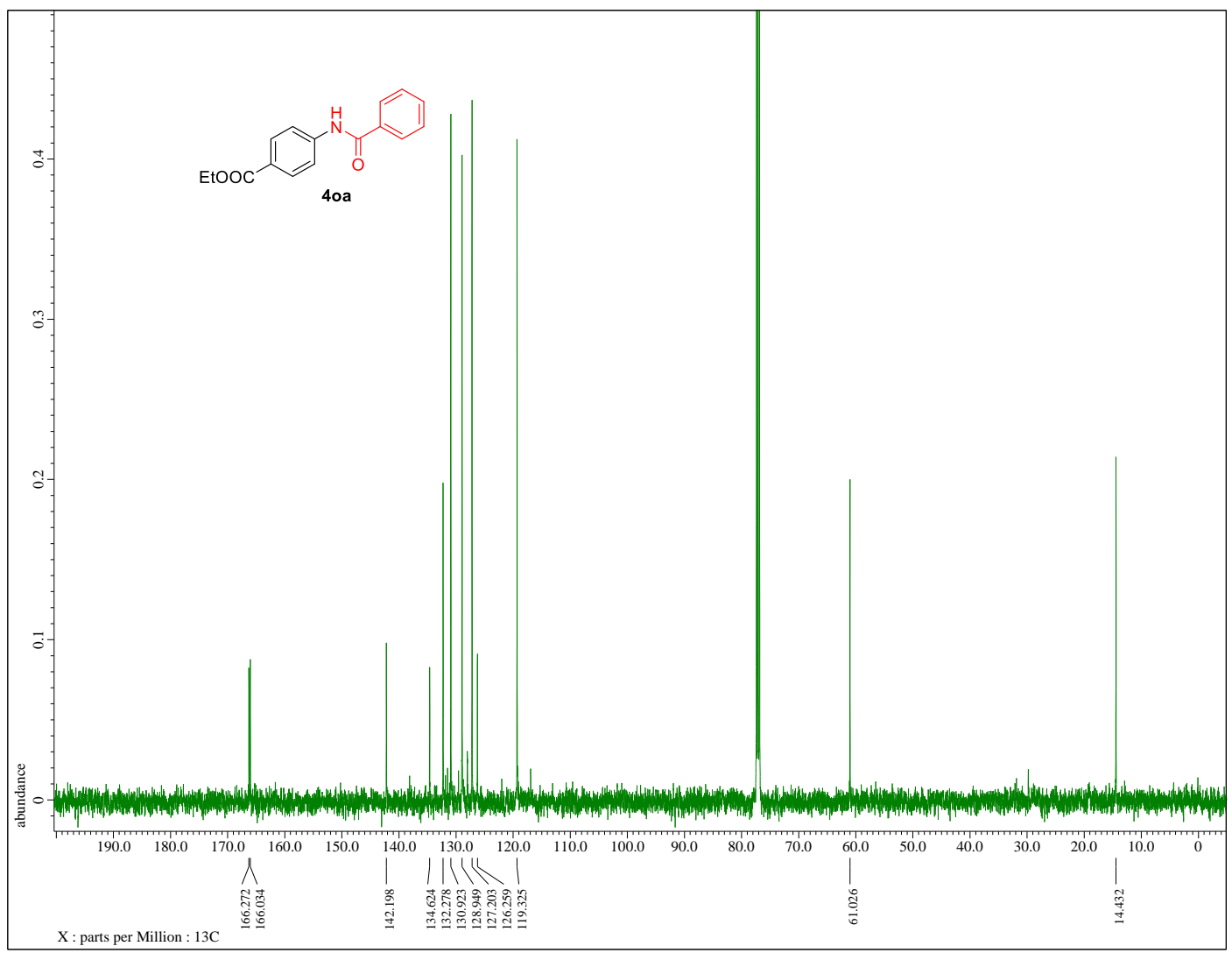

Figure S45-2. ${ }^{13} \mathrm{C}$ NMR spectrum of compound $40 a\left(125 \mathrm{MHz}, \mathrm{CDCl}_{3}\right)$ 


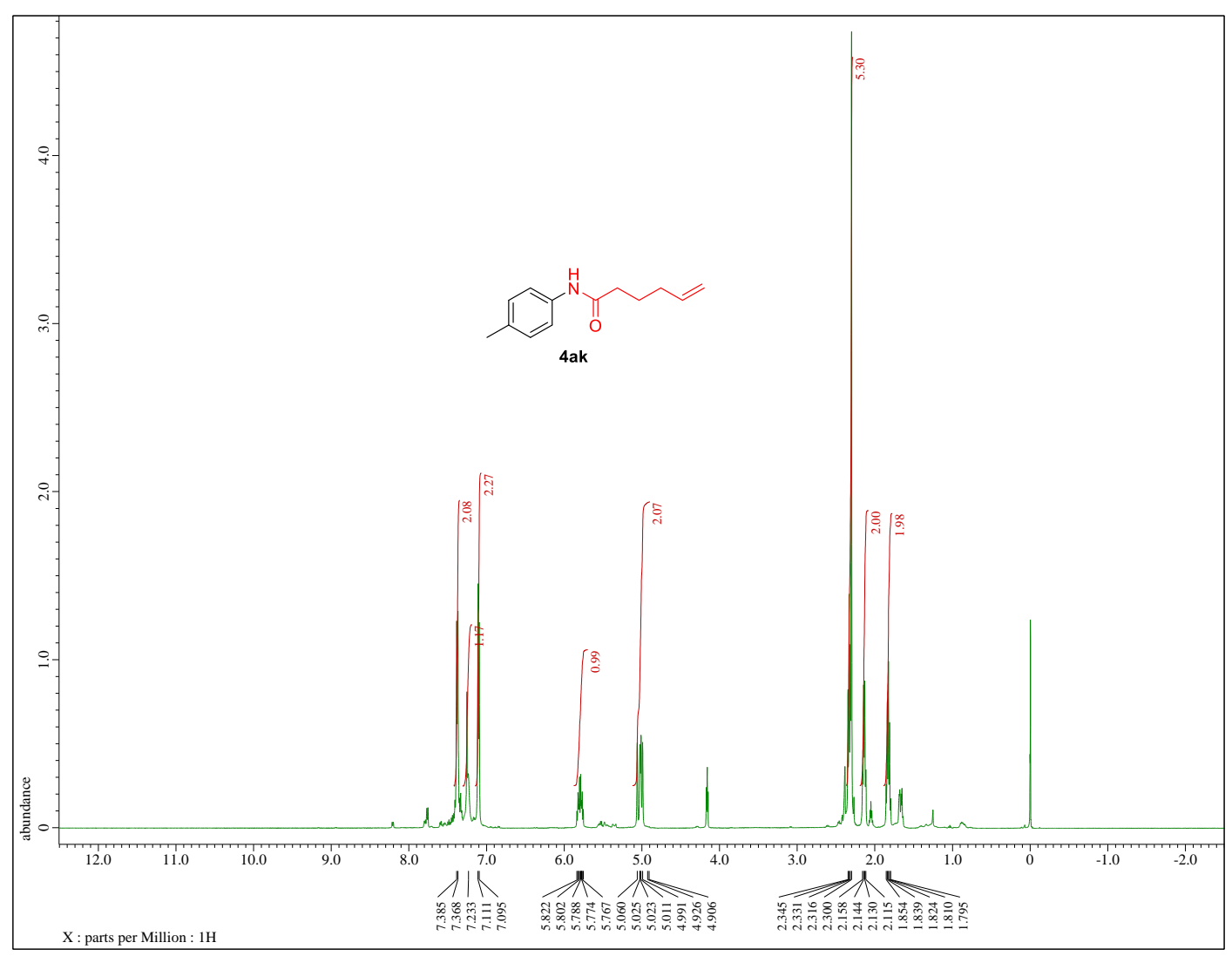

Figure S46-1. ${ }^{1} \mathrm{H}$ NMR spectrum of compound 4 ak $\left(500 \mathrm{MHz}, \mathrm{CDCl}_{3}\right)$

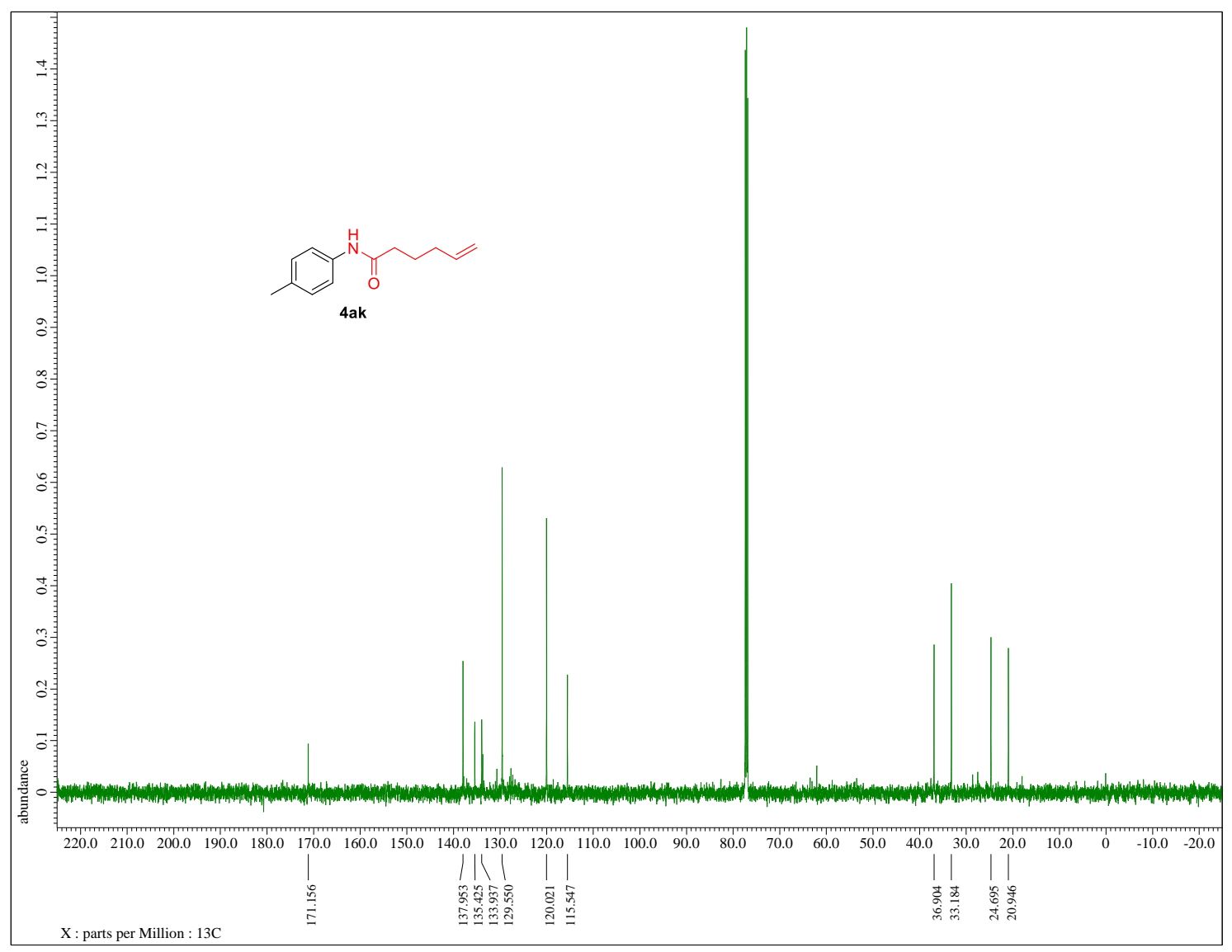

Figure S46-2. ${ }^{13} \mathrm{C}$ NMR spectrum of compound 4ak (125 MHz, $\left.\mathrm{CDCl}_{3}\right)$ 


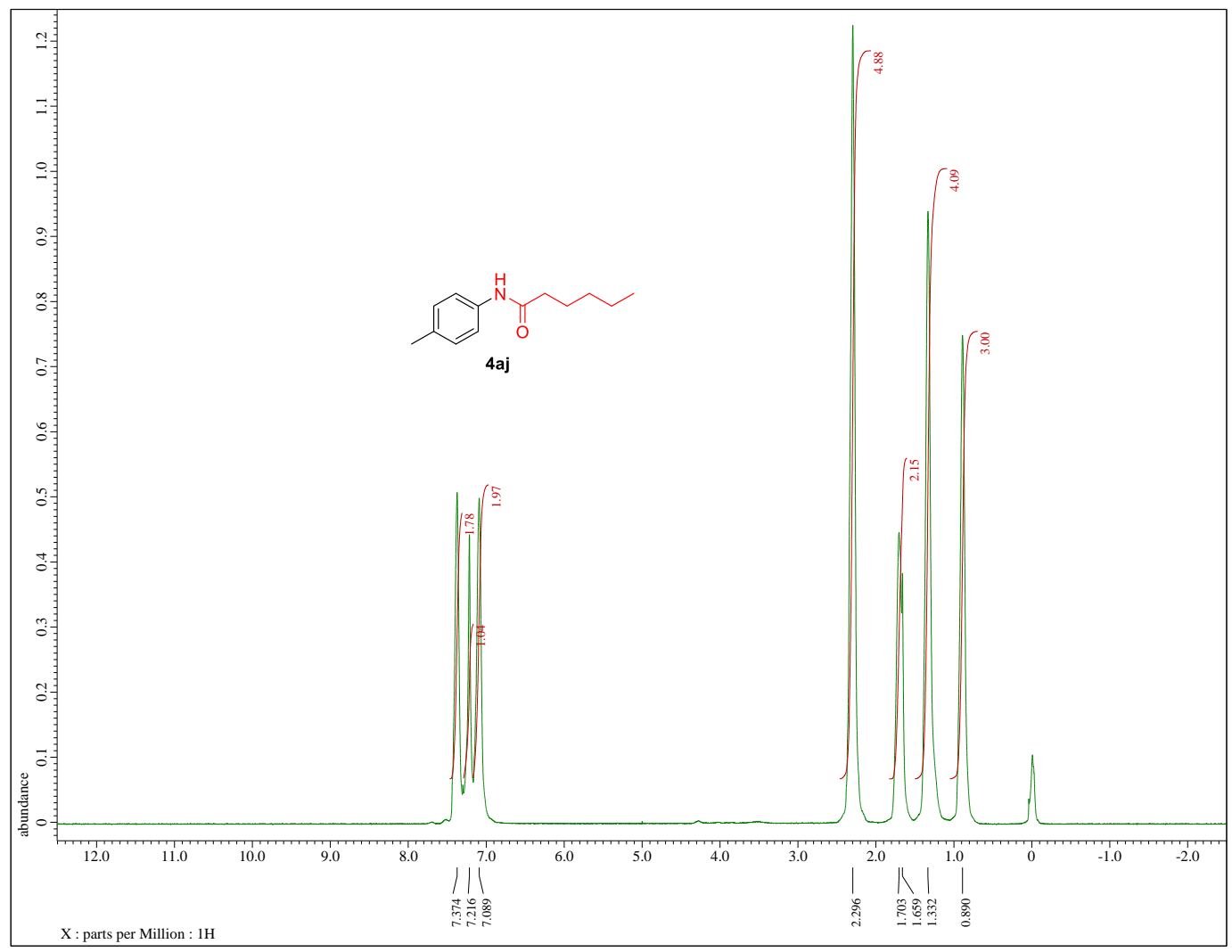

Figure S47-1. ${ }^{1} \mathrm{H}$ NMR spectrum of compound 4aj $\left(500 \mathrm{MHz}, \mathrm{CDCl}_{3}\right.$ )

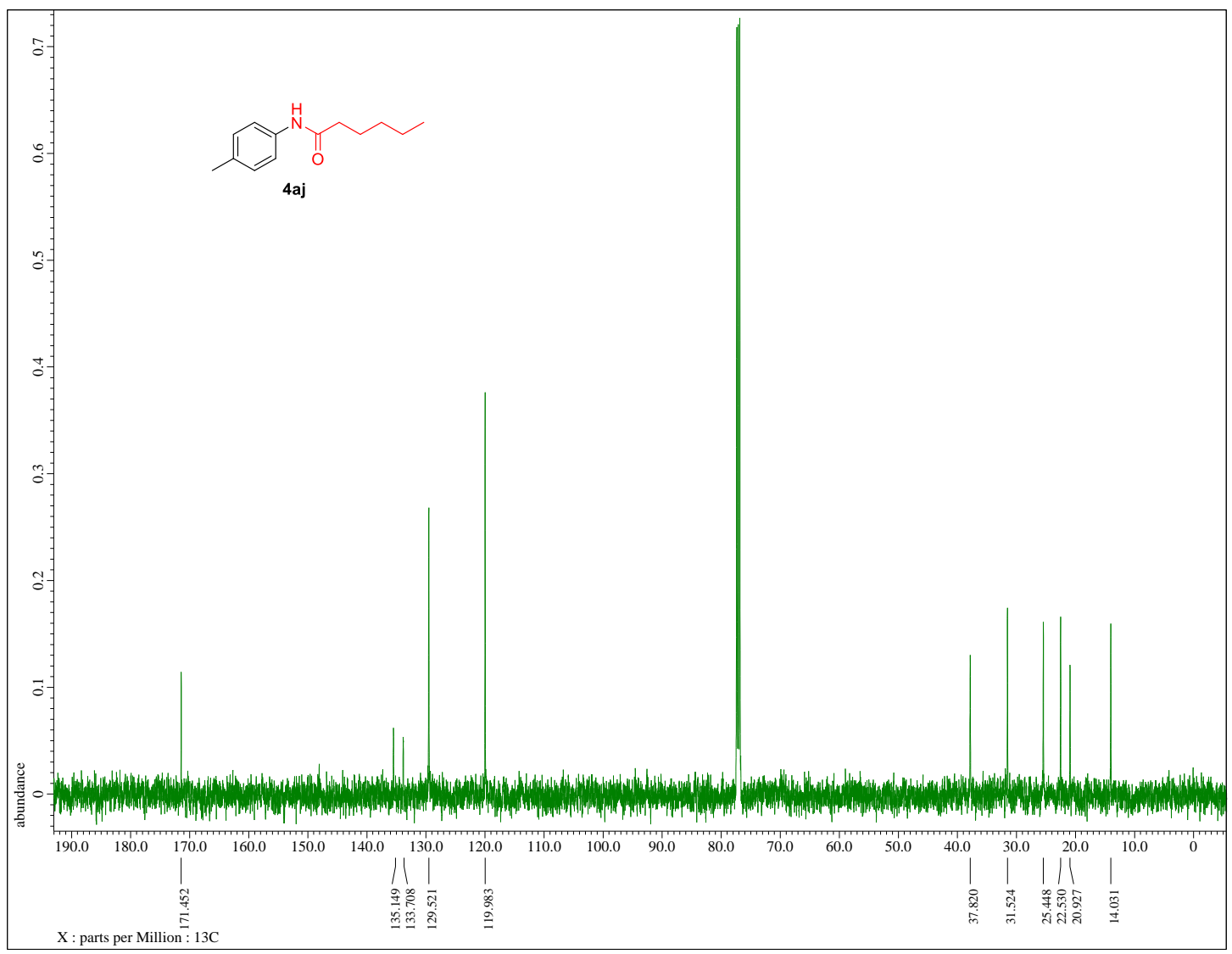

Figure S47-2. ${ }^{13} \mathrm{C}$ NMR spectrum of compound 4aj (125 $\left.\mathrm{MHz}, \mathrm{CDCl}_{3}\right)$ 


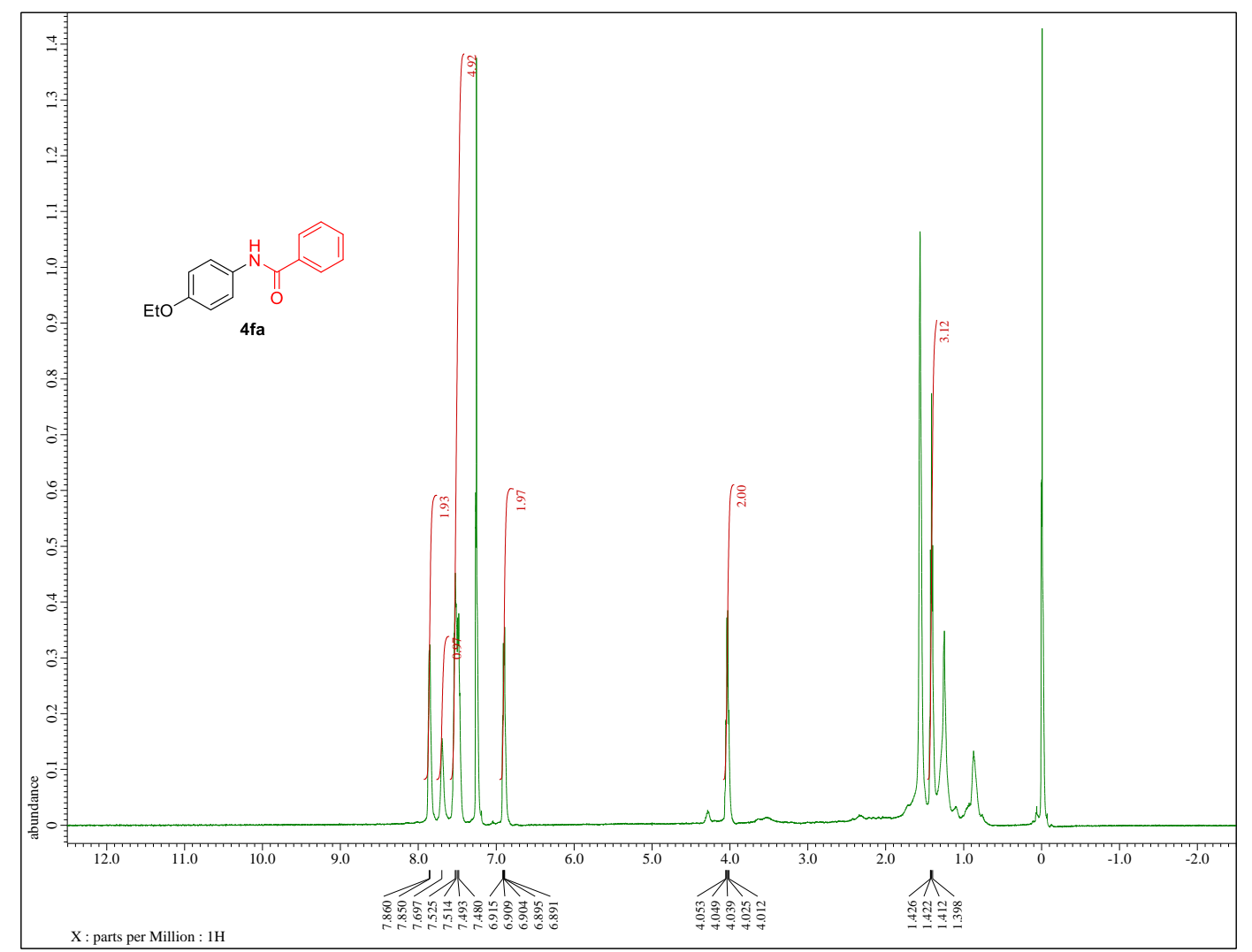

Figure S48-1. ${ }^{1} \mathrm{H}$ NMR spectrum of compound 4 fa $\left(500 \mathrm{MHz}, \mathrm{CDCl}_{3}\right)$

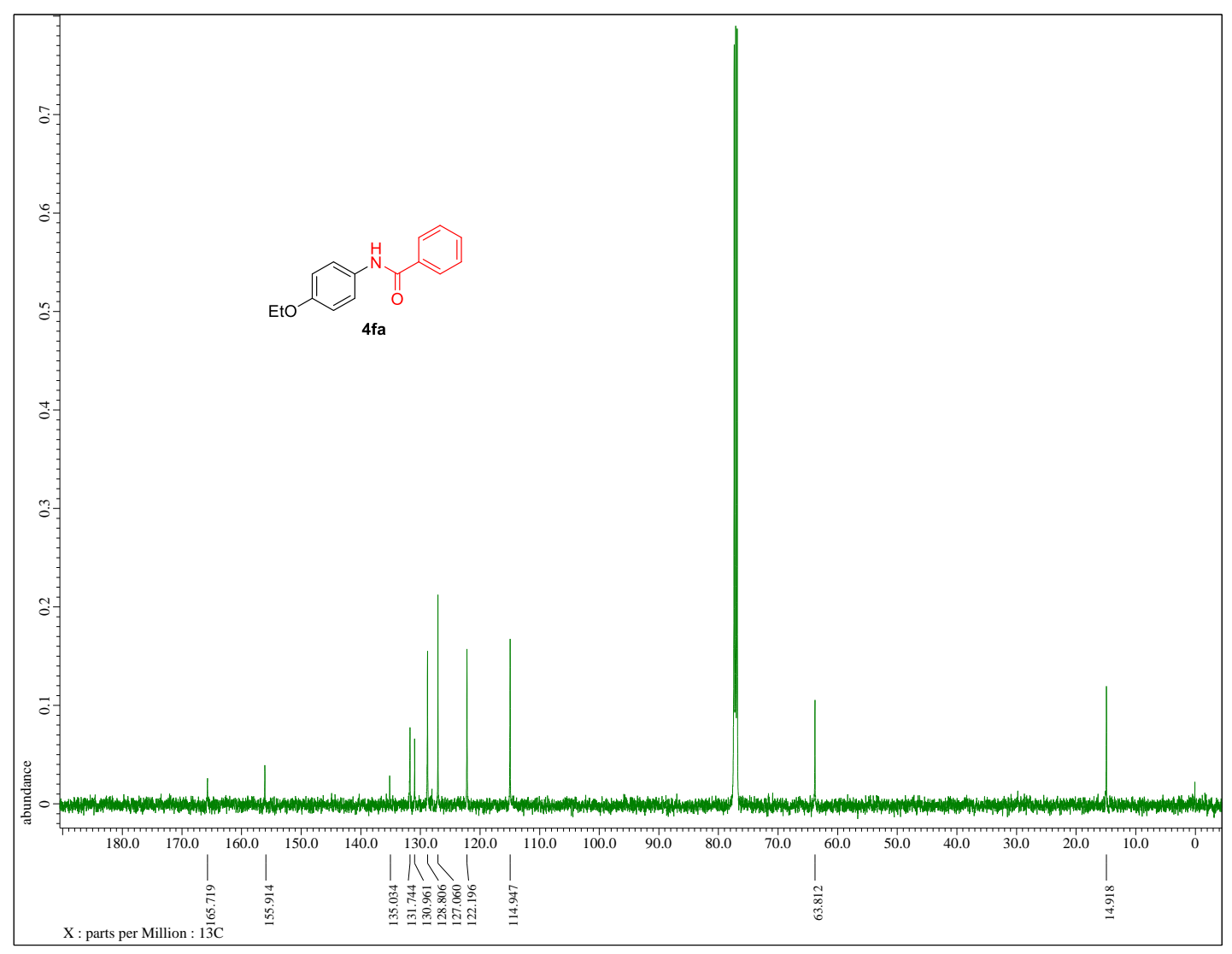

Figure S48-2. ${ }^{13} \mathrm{C}$ NMR spectrum of compound $\mathbf{4 f a}\left(125 \mathrm{MHz}, \mathrm{CDCl}_{3}\right)$ 


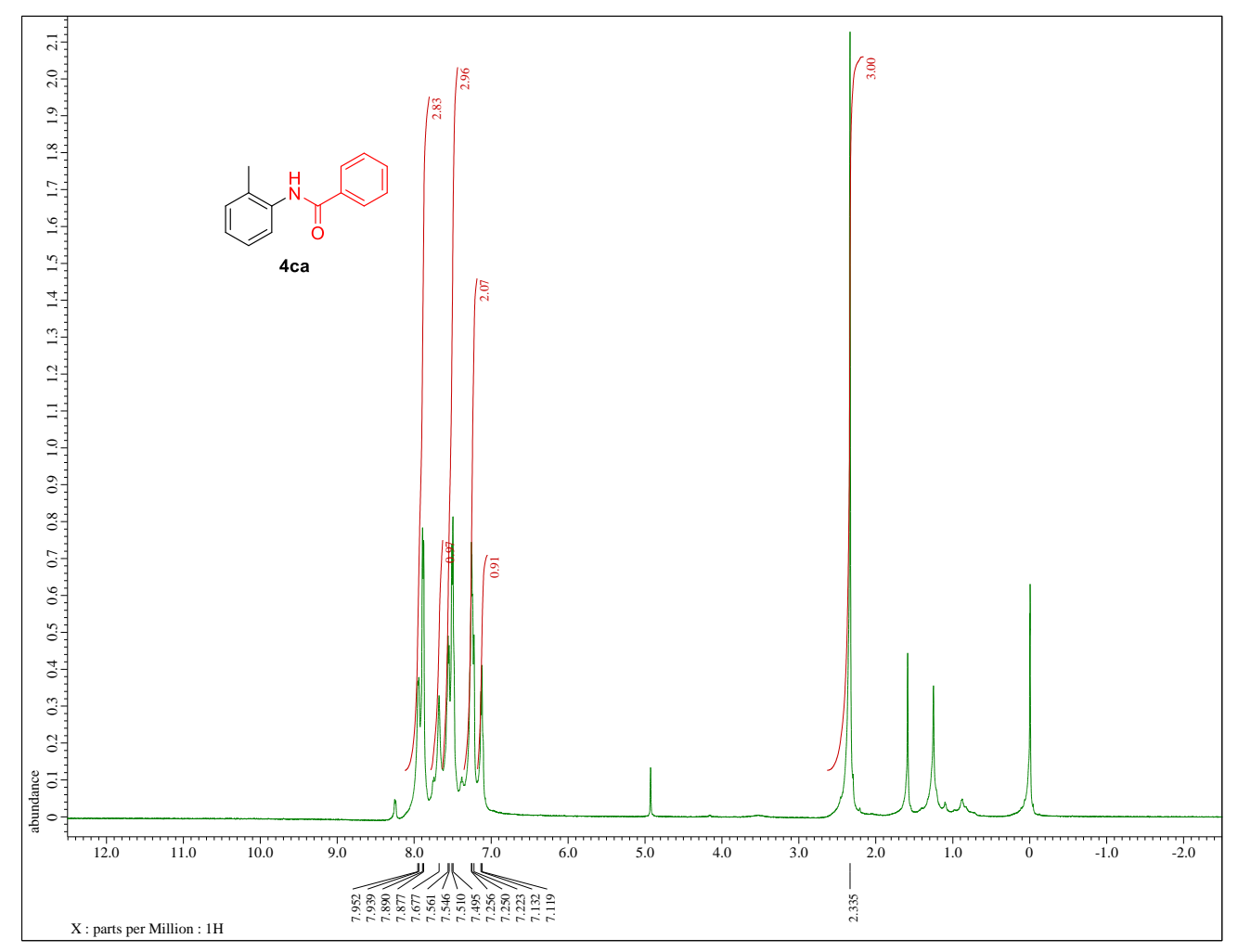

Figure S49-1. ${ }^{1} \mathrm{H}$ NMR spectrum of compound $4 \mathbf{c a}\left(500 \mathrm{MHz}, \mathrm{CDCl}_{3}\right)$

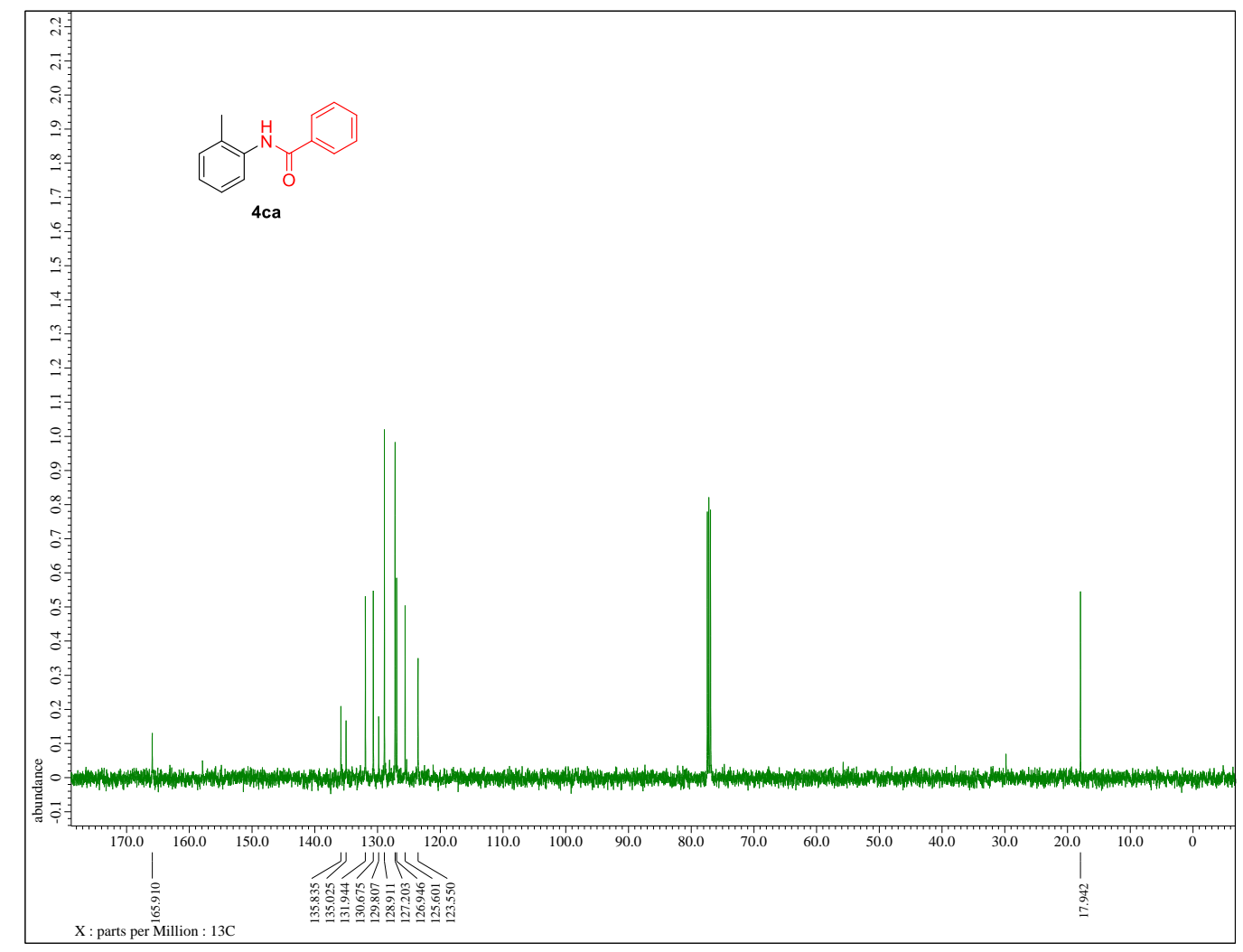

Figure S49-2. ${ }^{13} \mathrm{C}$ NMR spectrum of compound $\mathbf{4 c a}\left(125 \mathrm{MHz}, \mathrm{CDCl}_{3}\right)$ 


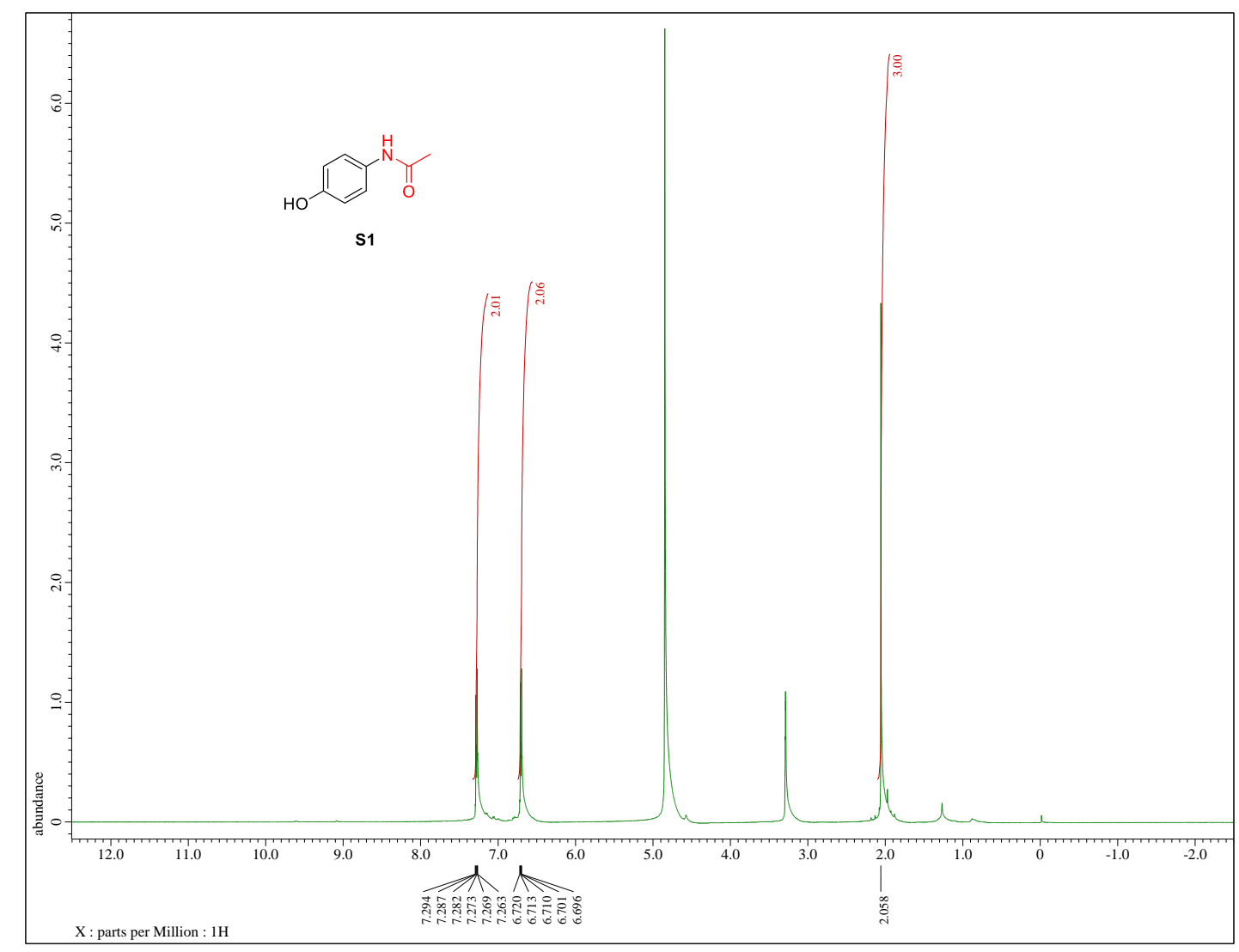

Figure S50-1. ${ }^{1} \mathrm{H}$ NMR spectrum of compound $\mathbf{S 1}\left(500 \mathrm{MHz}\right.$, Methanol-d $\left.\mathrm{d}_{3}\right)$

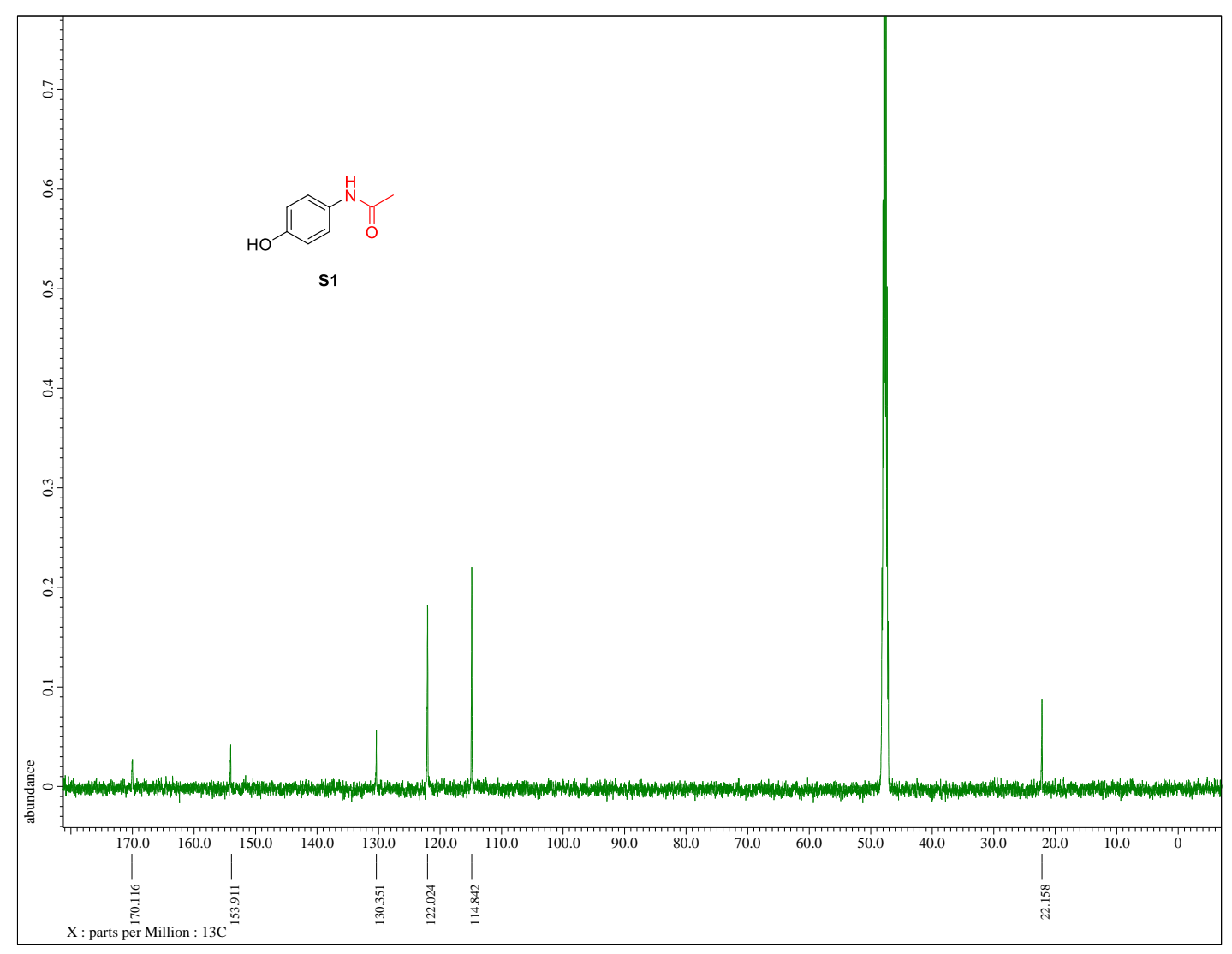

Figure S50-2. ${ }^{13} \mathrm{C}$ NMR spectrum of compound S1 (125 MHz, Methanol-d $\left.\mathrm{d}_{3}\right)$ 


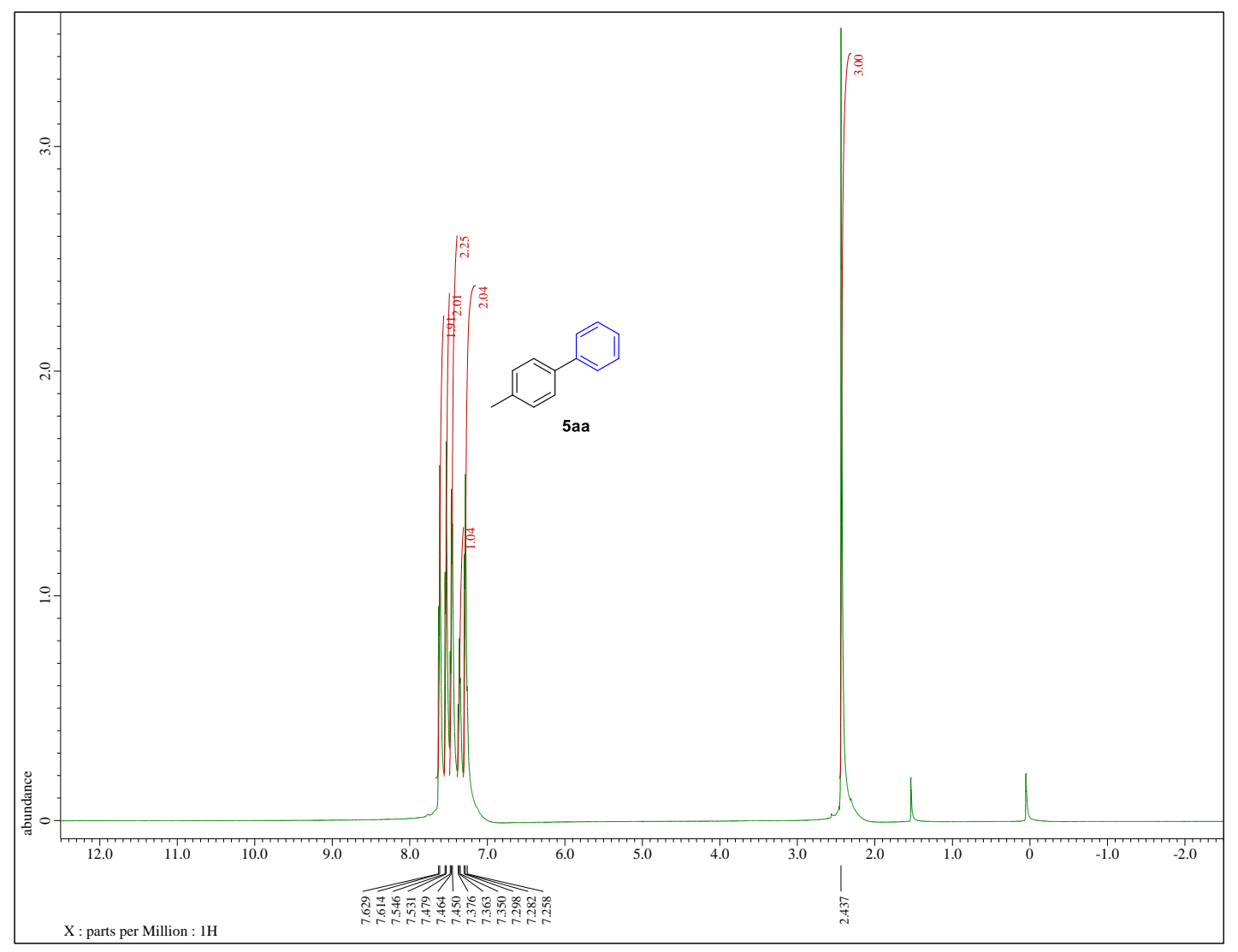

Figure S51-1. ${ }^{1} \mathrm{H}$ NMR spectrum of compound $\mathbf{5 a a}\left(500 \mathrm{MHz}, \mathrm{CDCl}_{3}\right)$

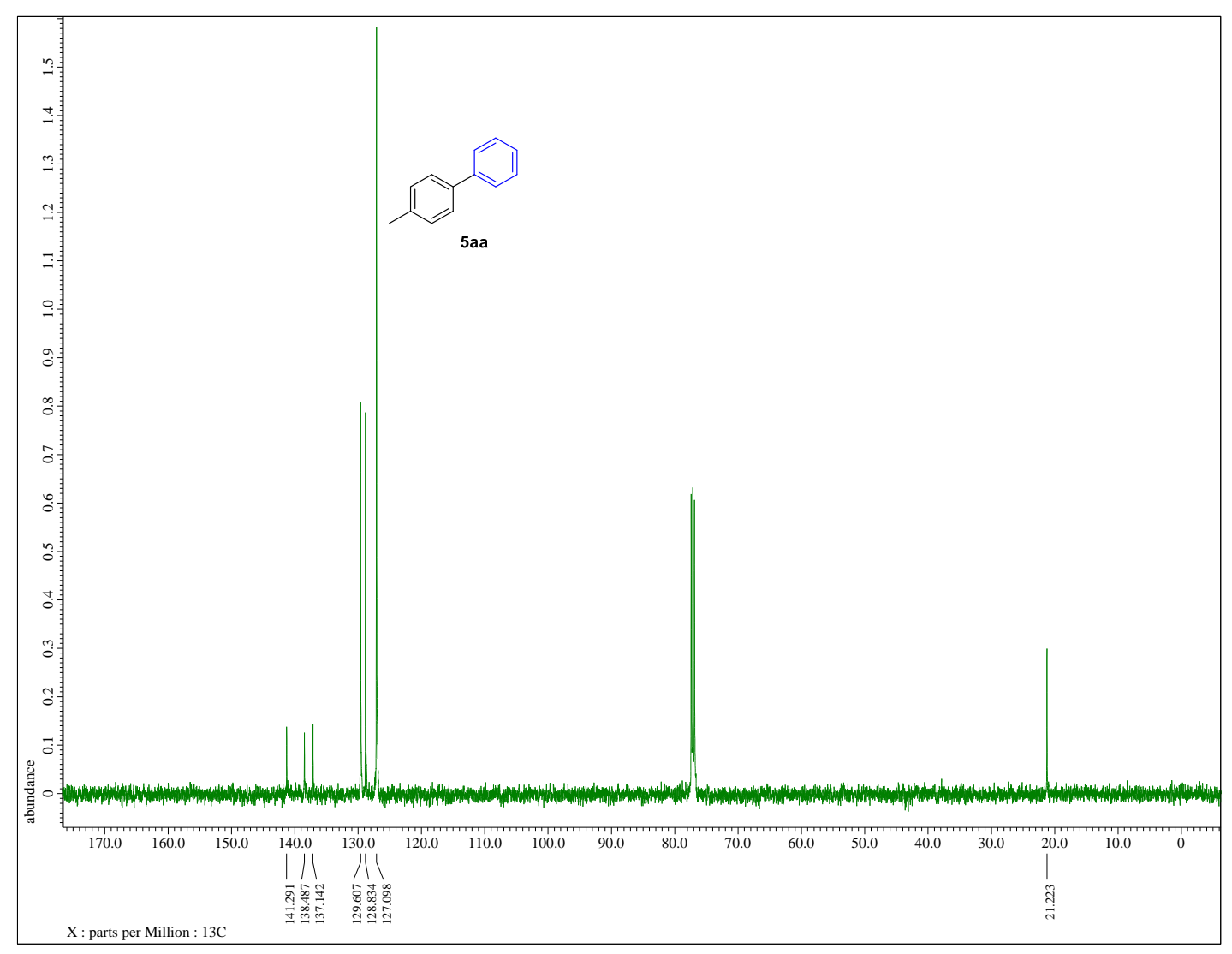

Figure S51-2. ${ }^{13} \mathrm{C}$ NMR spectrum of compound $\mathbf{5 a a}\left(125 \mathrm{MHz}, \mathrm{CDCl}_{3}\right)$ 


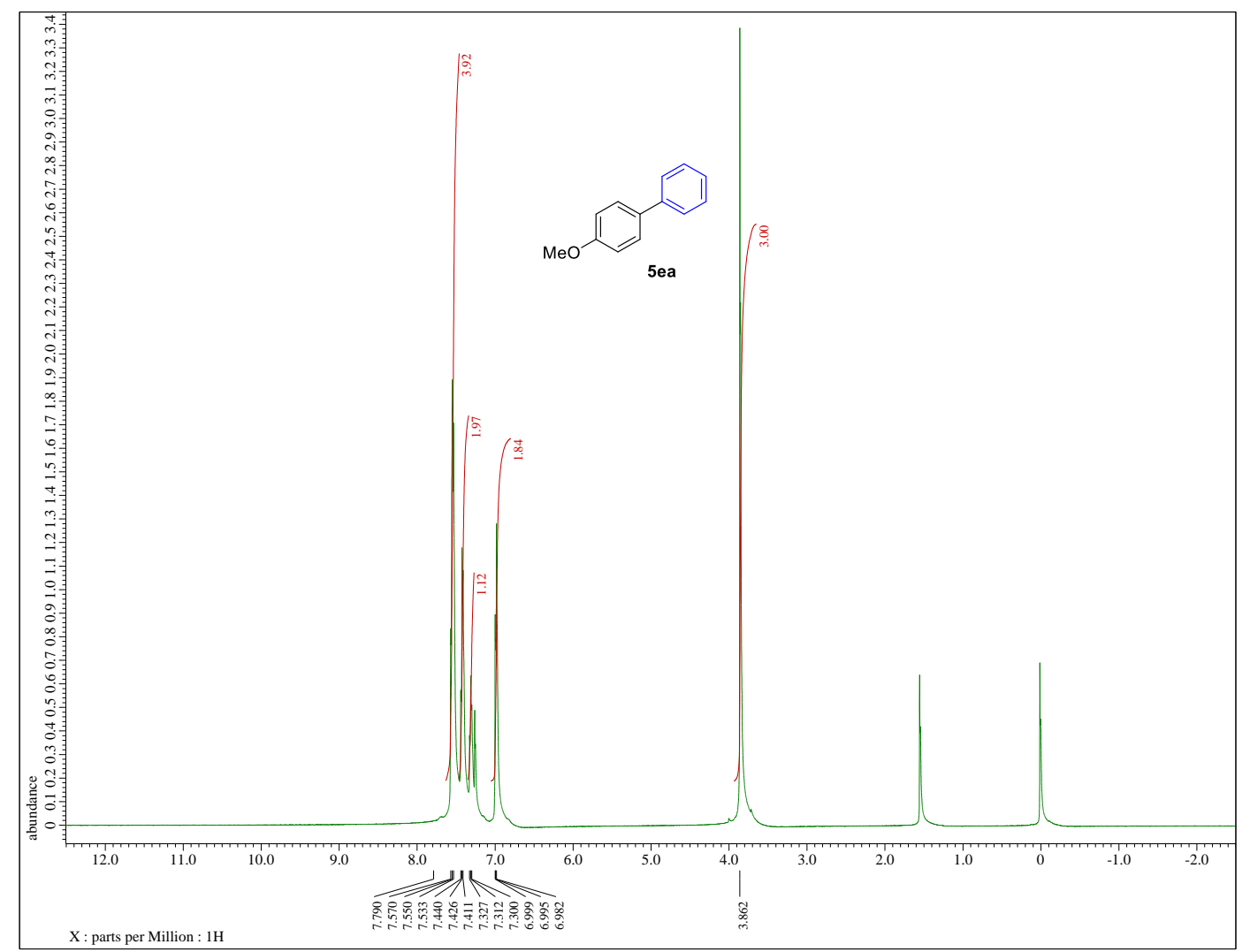

Figure S52-1. ${ }^{1} \mathrm{H}$ NMR spectrum of compound 5 ea $\left(500 \mathrm{MHz}, \mathrm{CDCl}_{3}\right)$

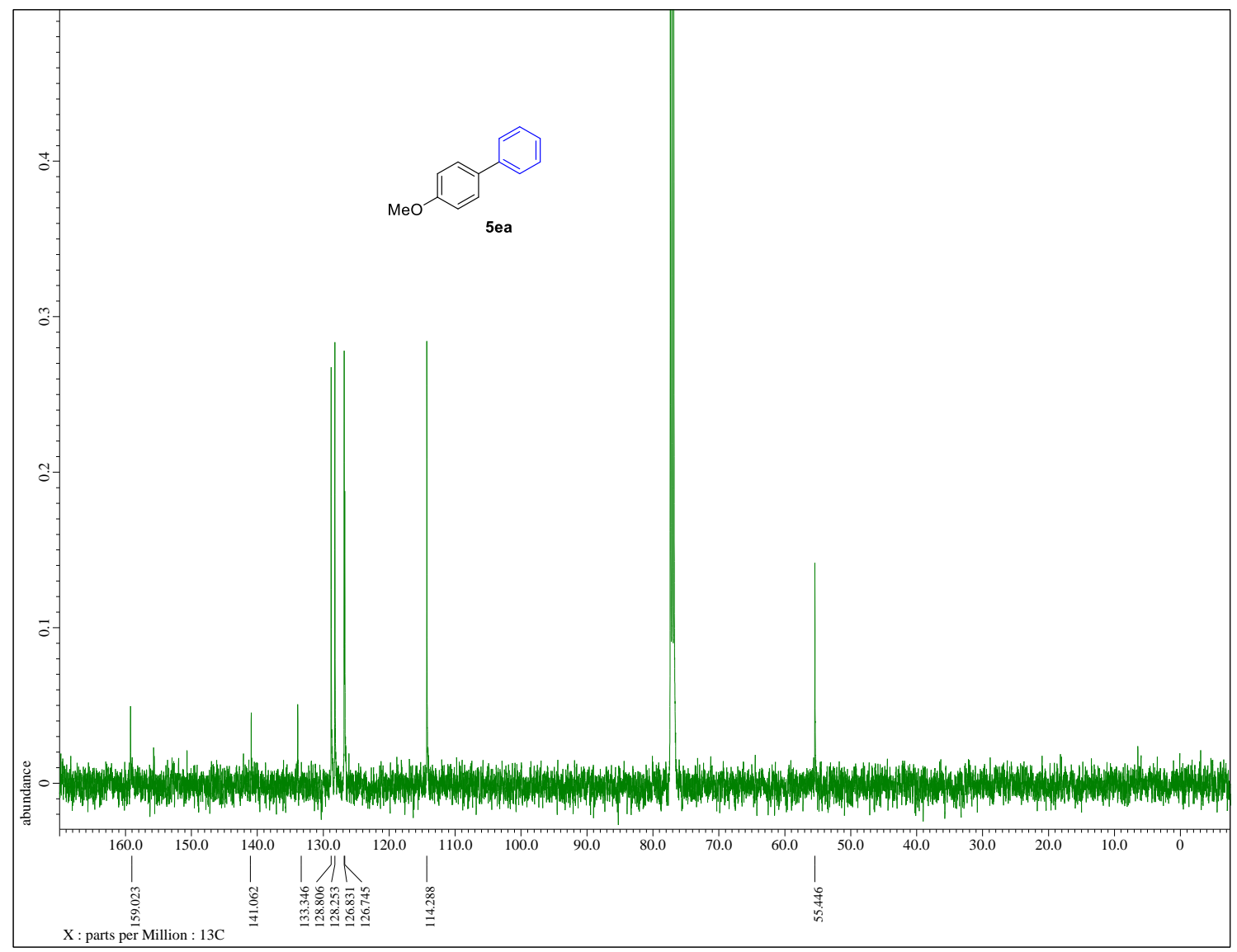

Figure S52-2. ${ }^{13} \mathrm{C}$ NMR spectrum of compound 5ea $\left(125 \mathrm{MHz}, \mathrm{CDCl}_{3}\right)$ 


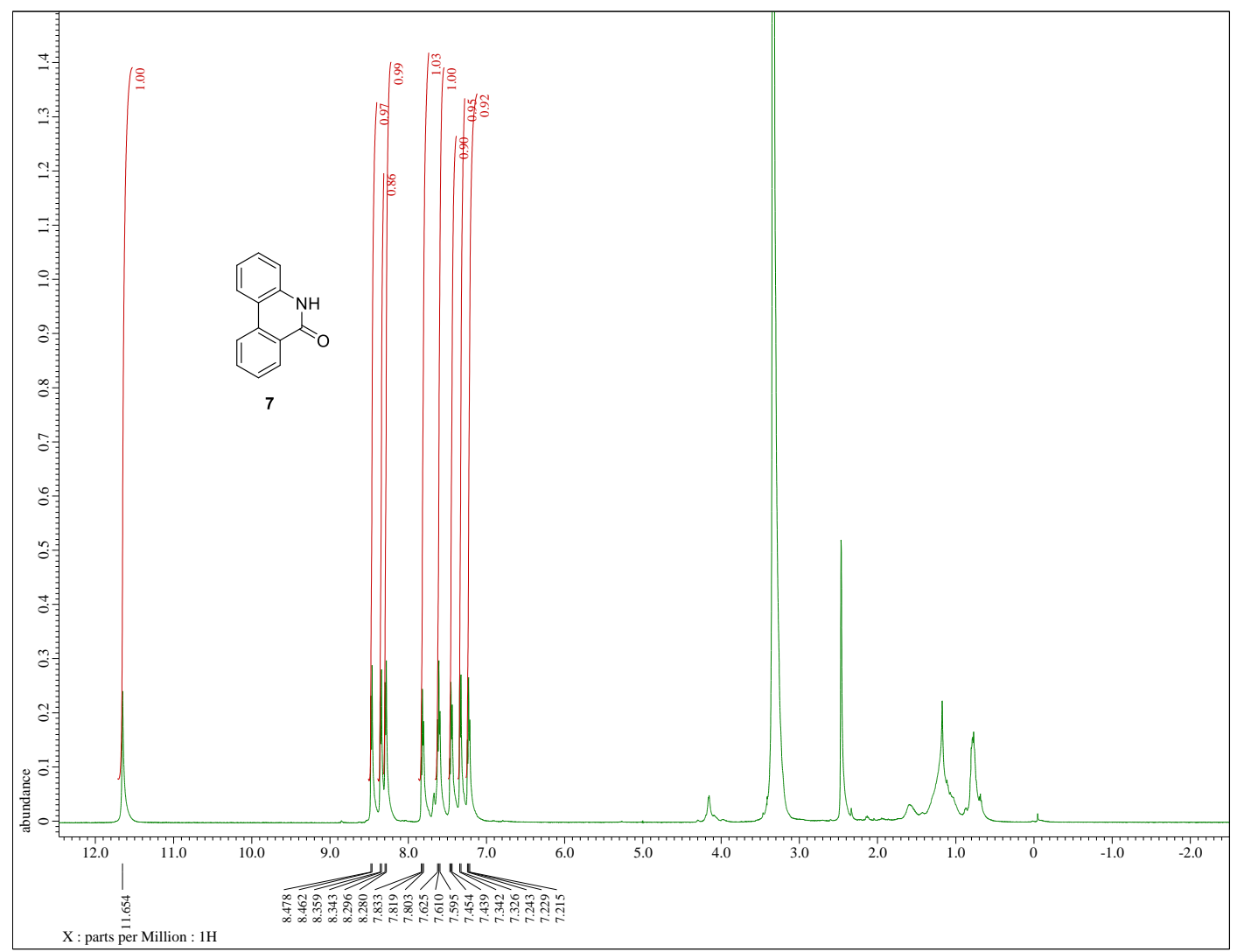

Figure S53-1. ${ }^{1} \mathrm{H}$ NMR spectrum of compound 7 (500 MHz, DMSO-d $\left.\mathrm{d}_{6}\right)$

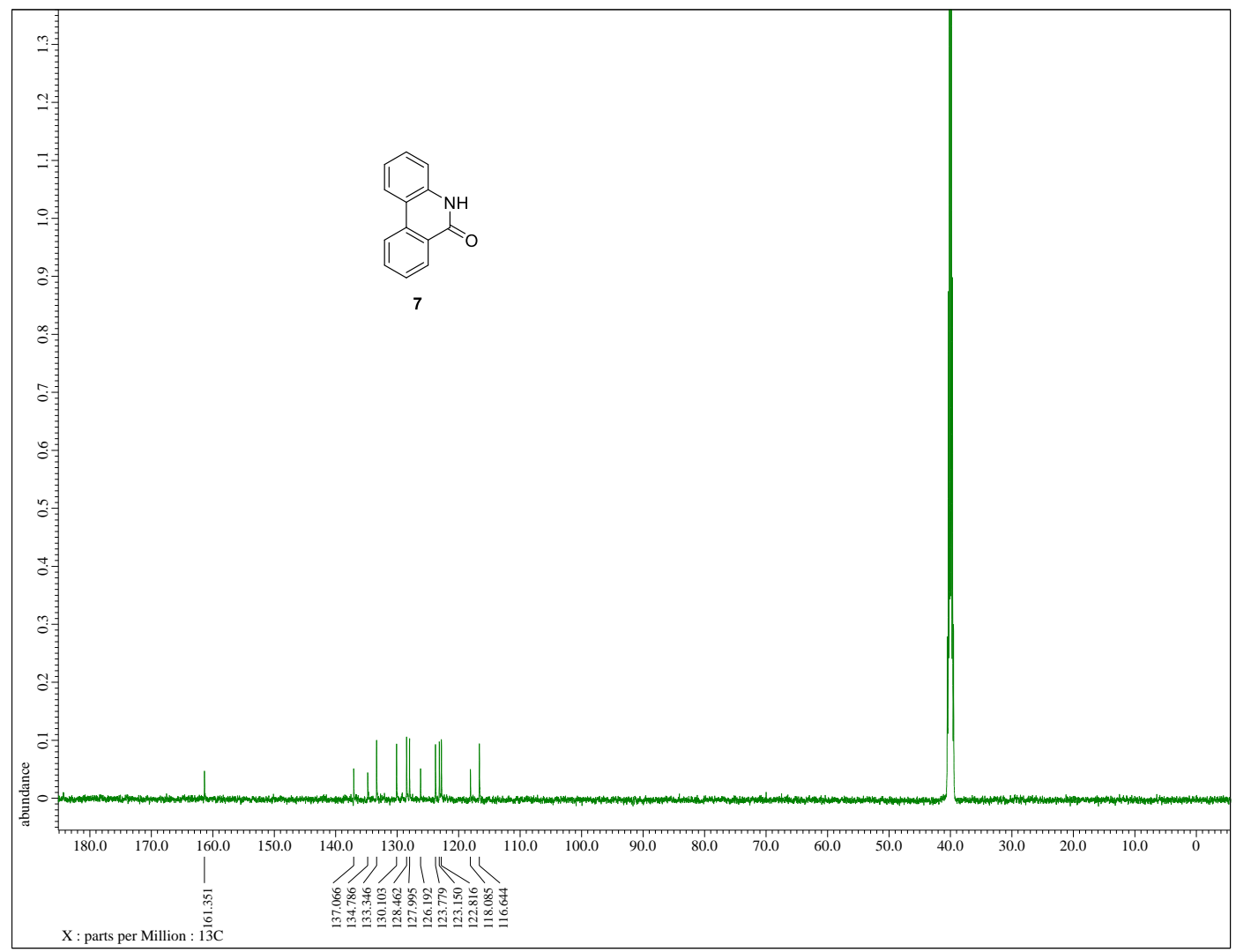

Figure S53-2. ${ }^{13} \mathrm{C}$ NMR spectrum of compound 7 (125 MHz, DMSO-d 6 ) 


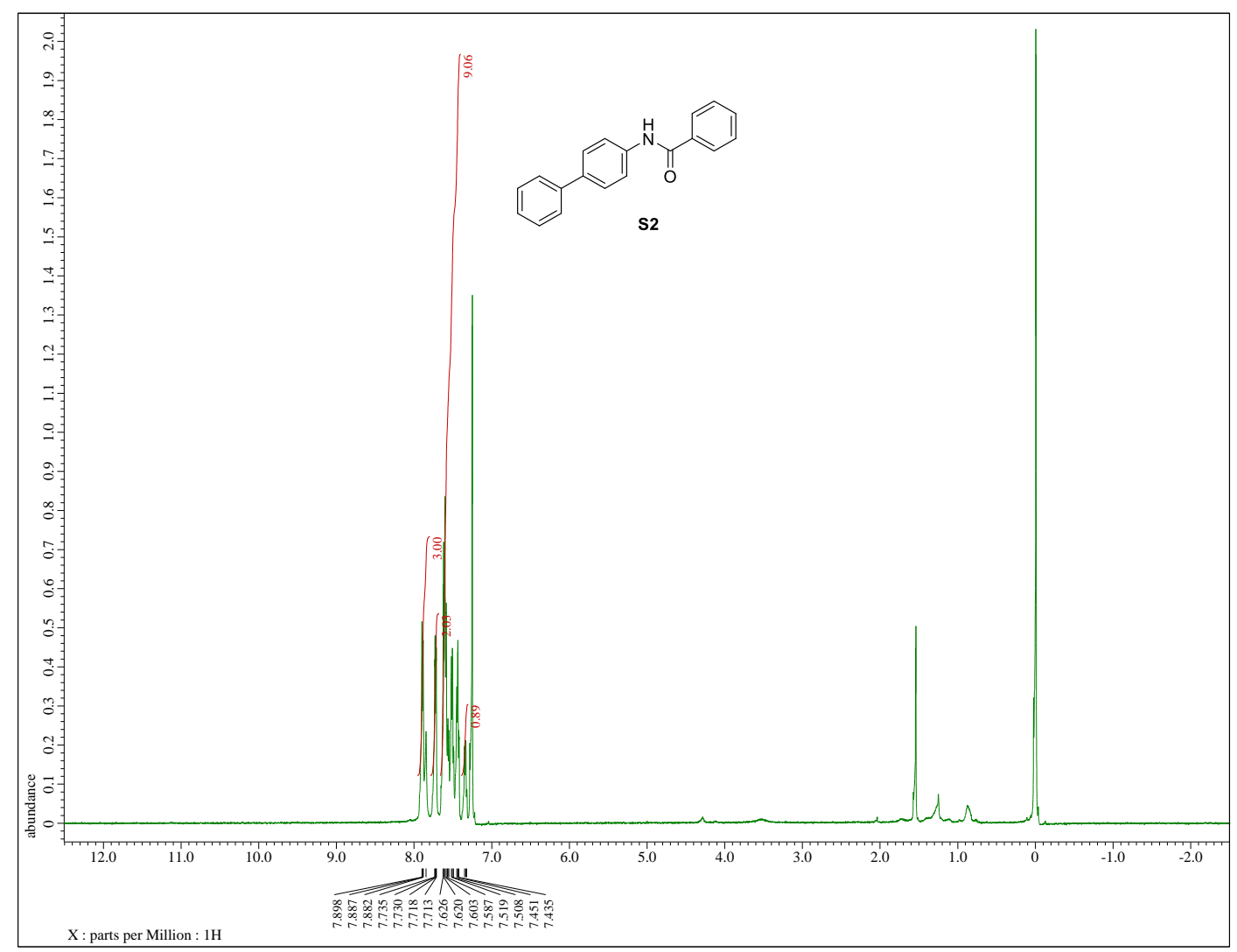

Figure S54-1. ${ }^{1} \mathrm{H}$ NMR spectrum of compound $\mathbf{S 2}\left(500 \mathrm{MHz}, \mathrm{CDCl}_{3}\right)$

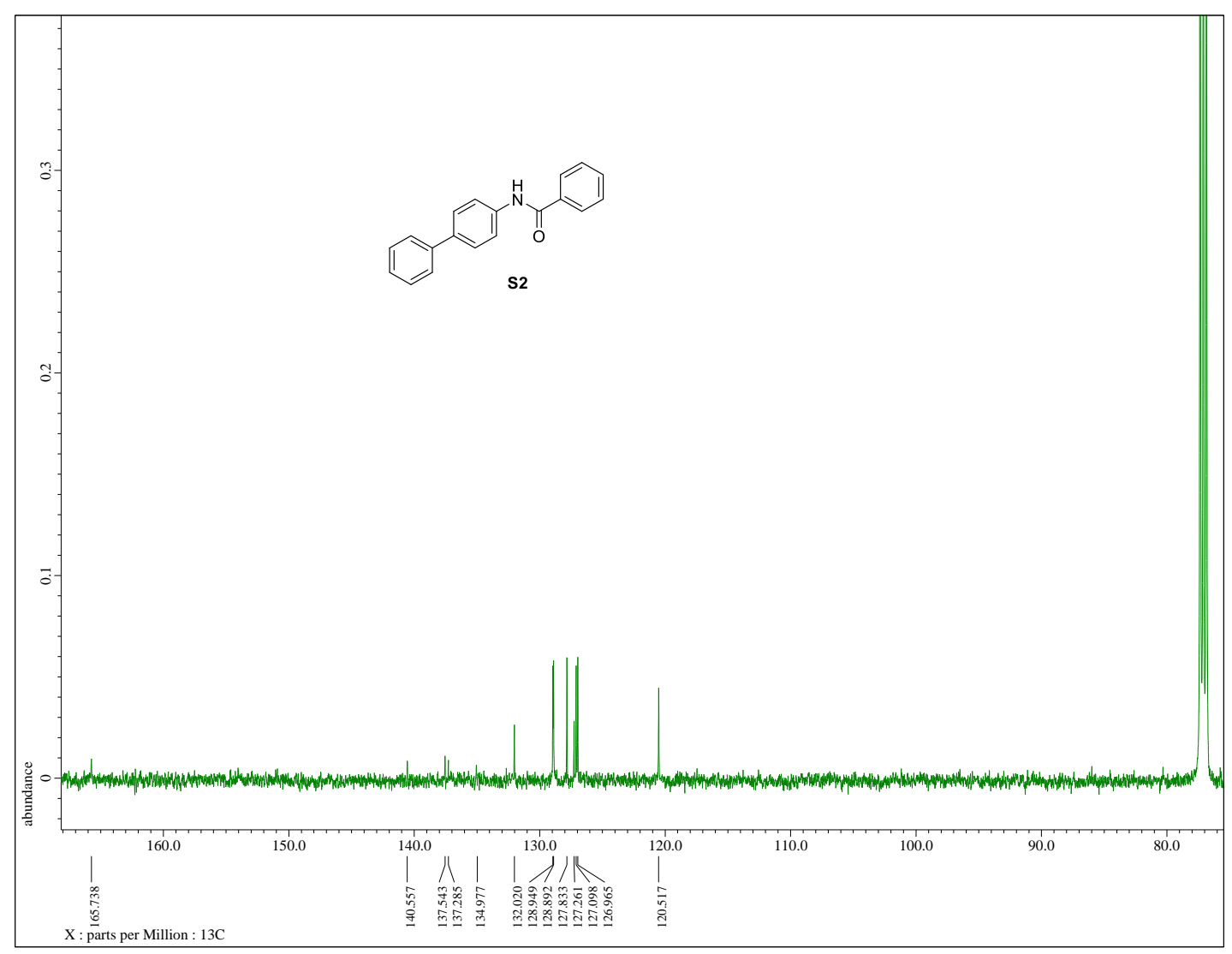

Figure S54-2. ${ }^{13} \mathrm{C}$ NMR spectrum of compound $\mathbf{S 2}\left(125 \mathrm{MHz}, \mathrm{CDCl}_{3}\right)$ 


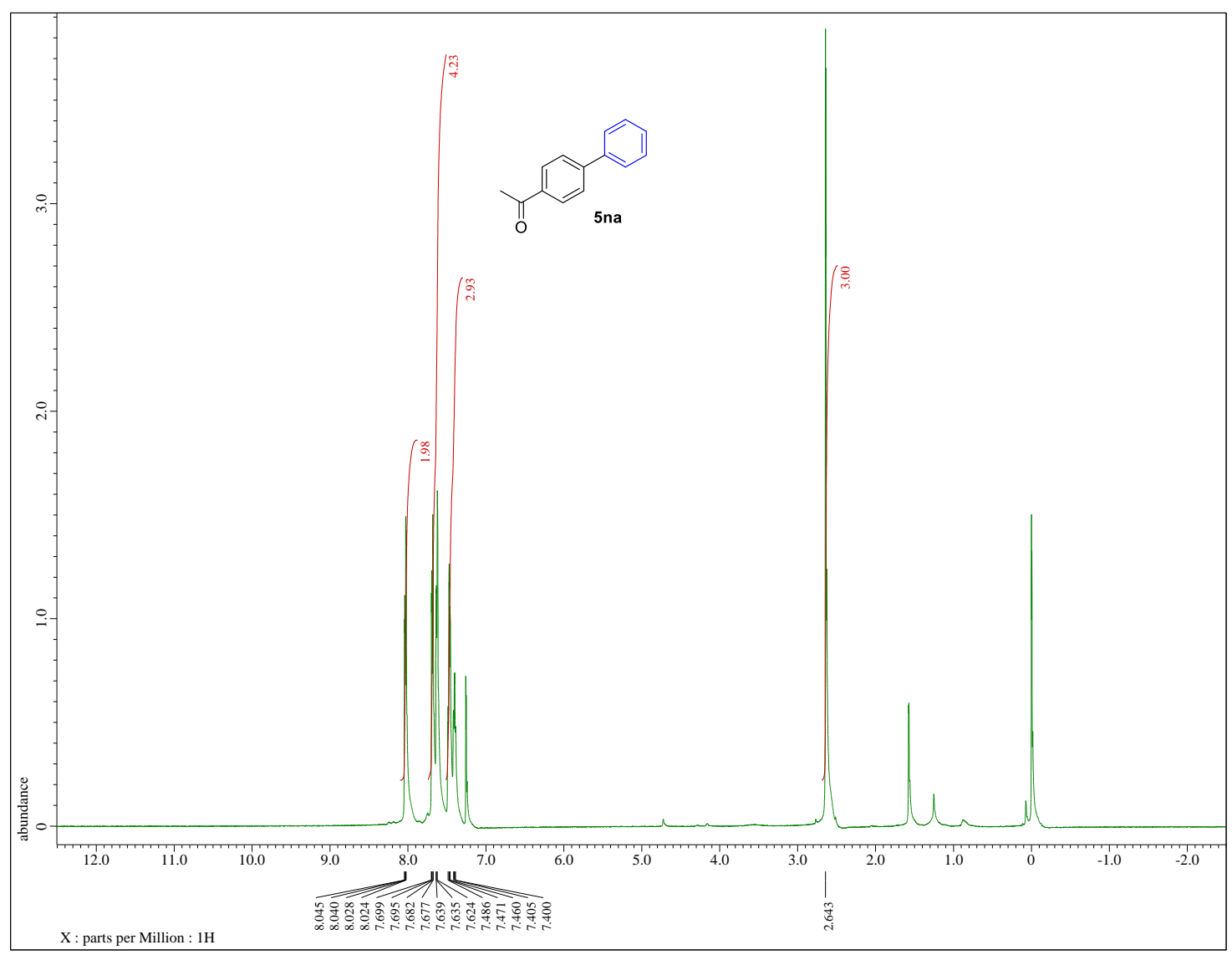

Figure S55-1. ${ }^{1} \mathrm{H}$ NMR spectrum of compound 5 na $\left(500 \mathrm{MHz}, \mathrm{CDCl}_{3}\right)$

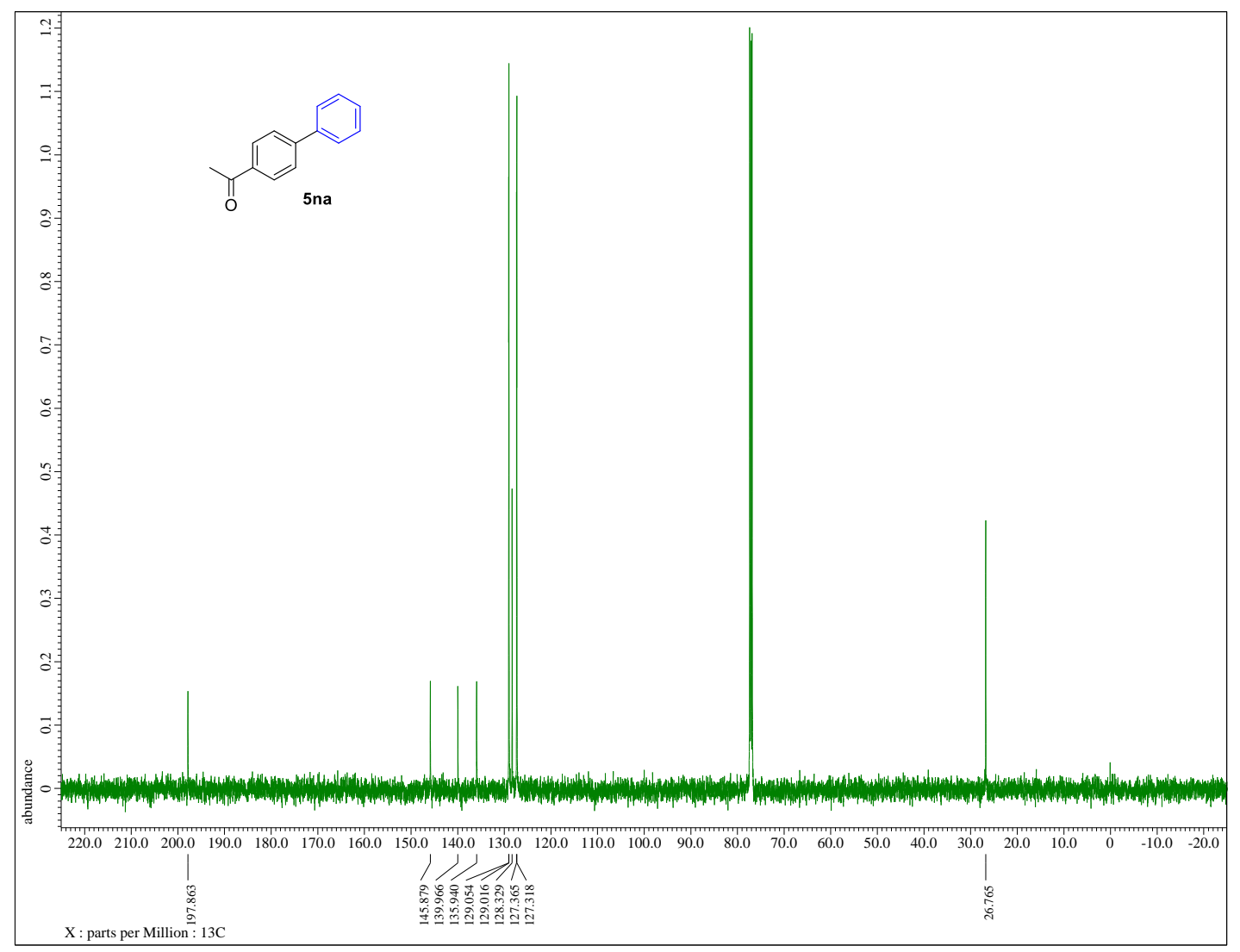

Figure S55-2. ${ }^{13} \mathrm{C}$ NMR spectrum of compound 5 na $\left(125 \mathrm{MHz}, \mathrm{CDCl}_{3}\right)$ 


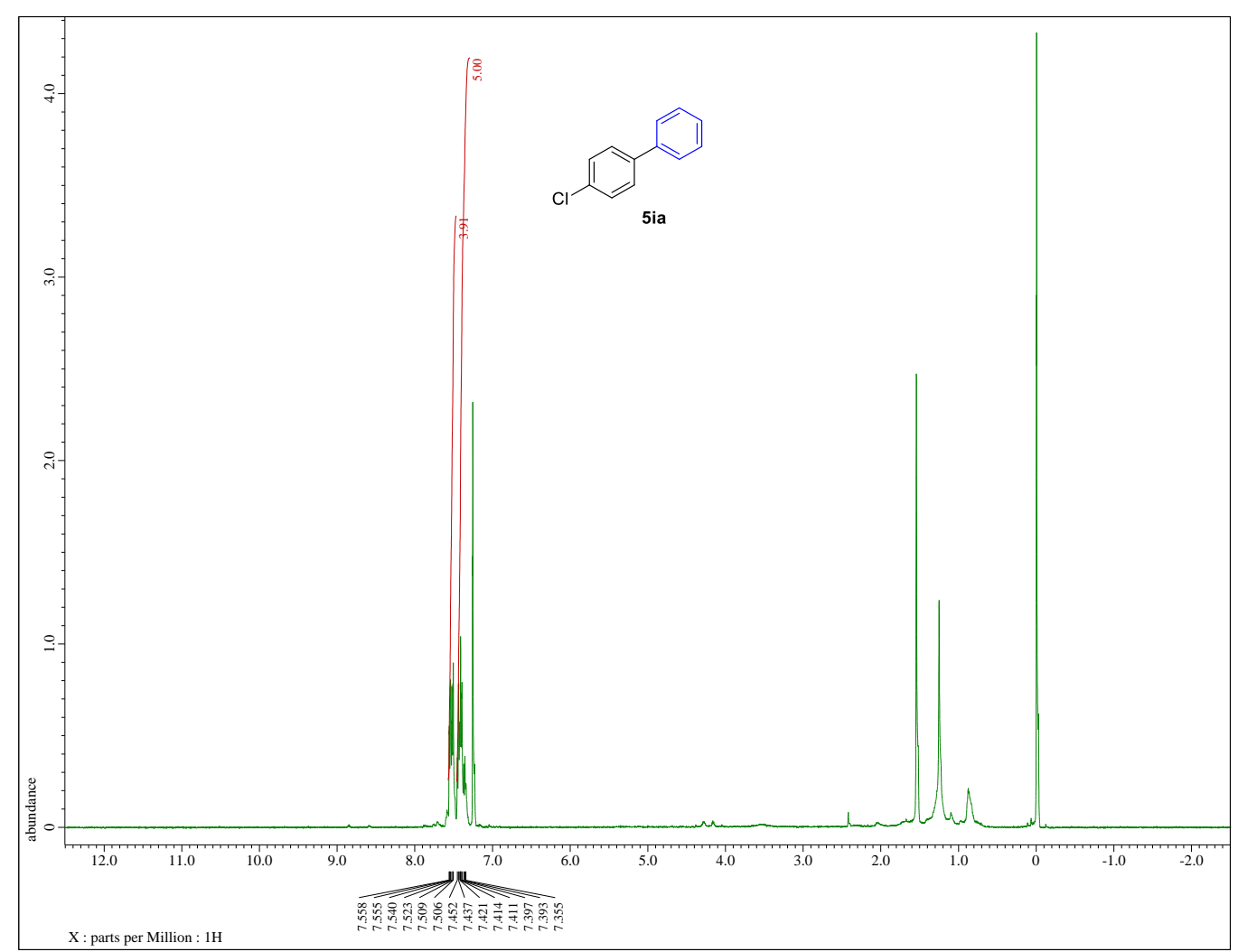

Figure S56-1. ${ }^{1} \mathrm{H}$ NMR spectrum of compound $5 i a\left(500 \mathrm{MHz}, \mathrm{CDCl}_{3}\right)$

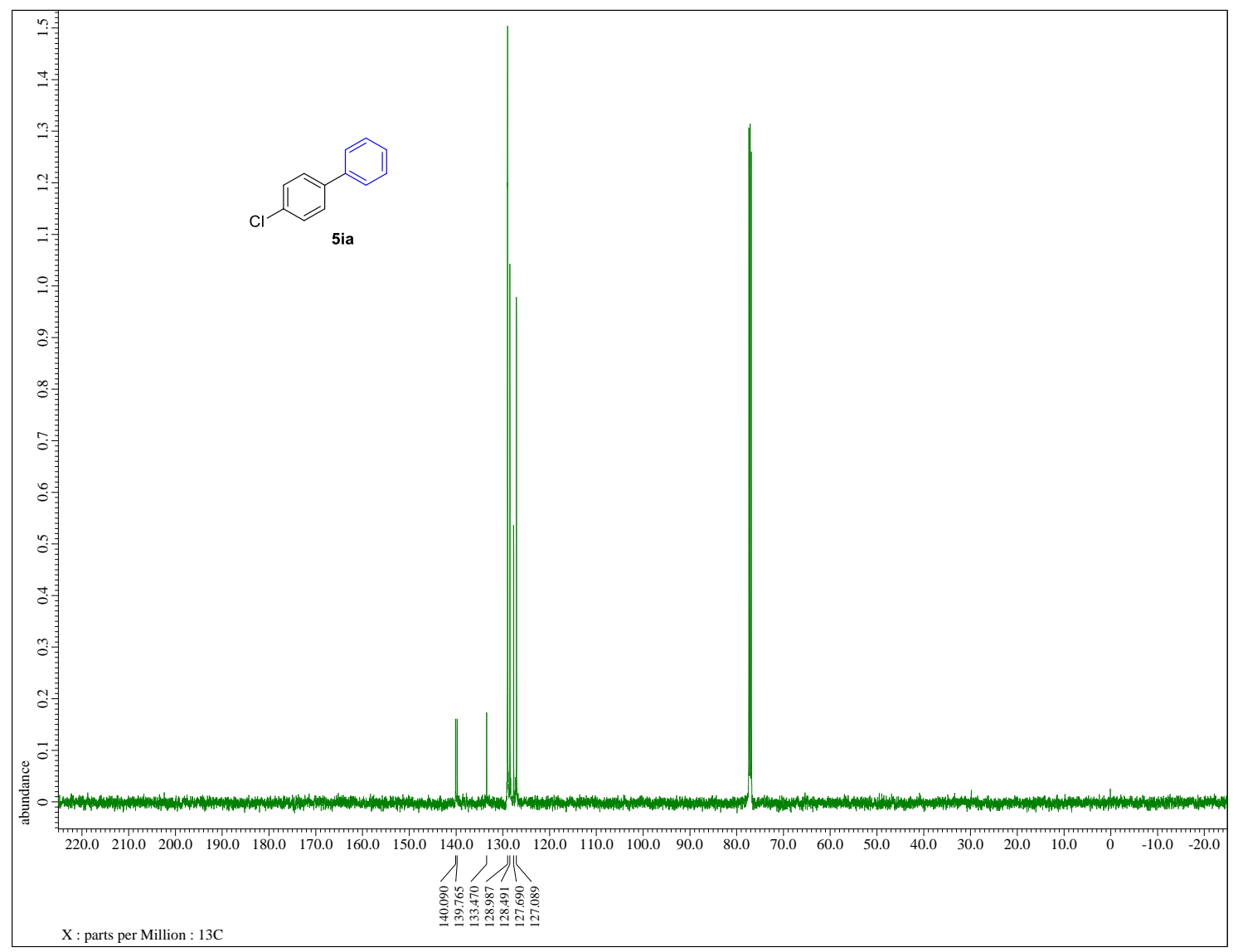

Figure S56-2. ${ }^{13} \mathrm{C} \mathrm{NMR}$ spectrum of compound 5 ia $\left(125 \mathrm{MHz}, \mathrm{CDCl}_{3}\right)$ 


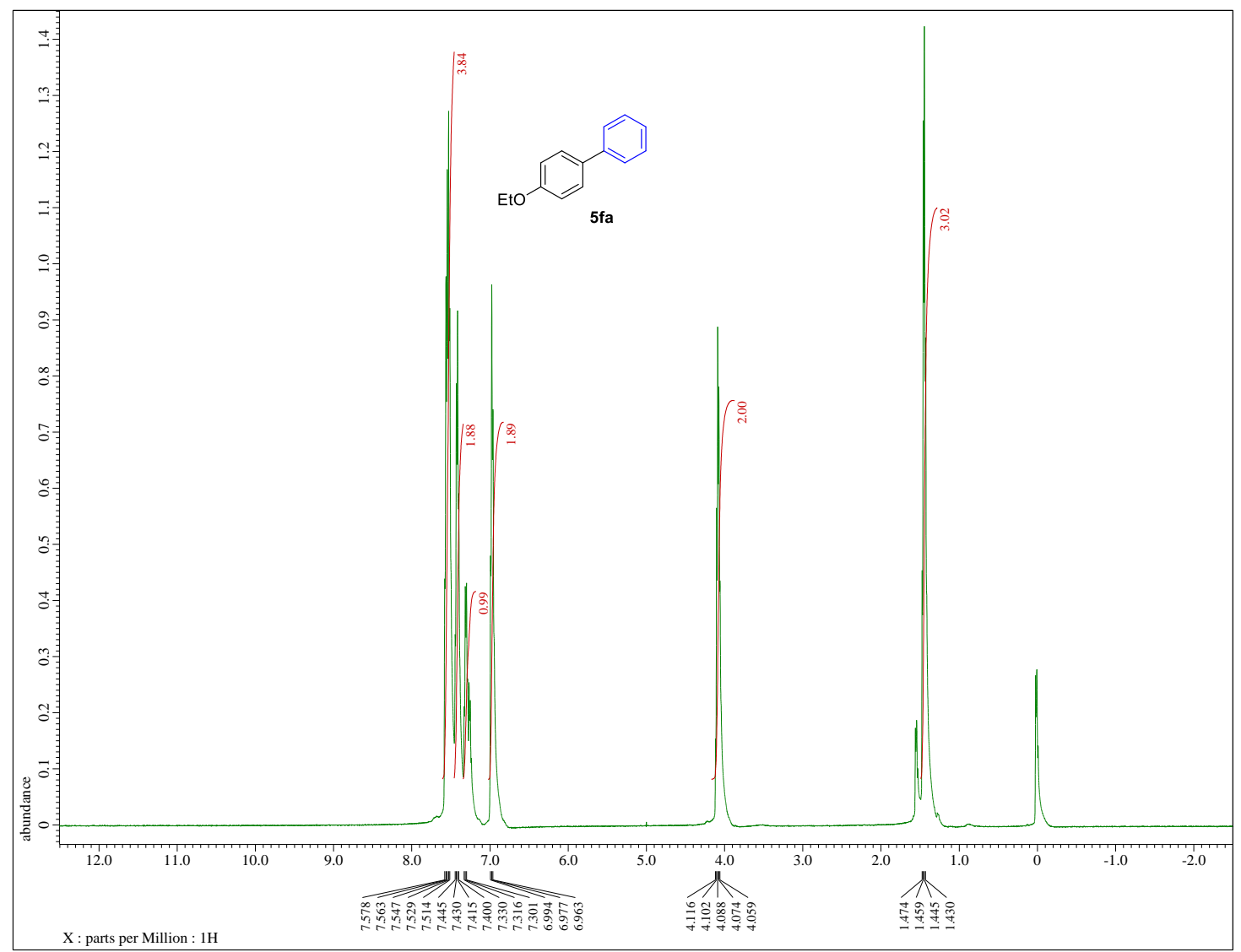

Figure S57-1. ${ }^{1} \mathrm{H}$ NMR spectrum of compound $5 \mathbf{f a}\left(500 \mathrm{MHz}, \mathrm{CDCl}_{3}\right)$

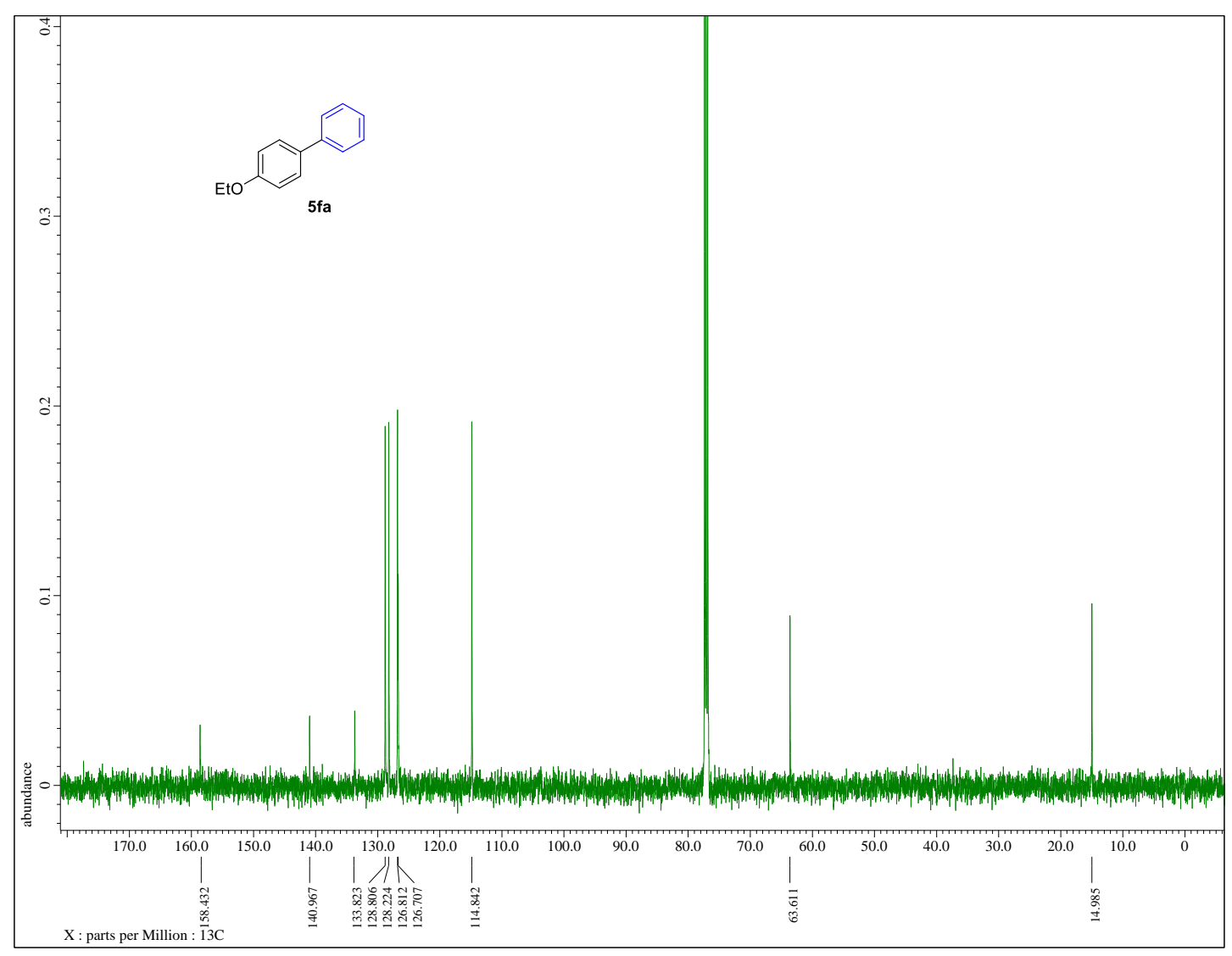

Figure S57-2. ${ }^{13} \mathrm{C}$ NMR spectrum of compound $\mathbf{5 f a}\left(125 \mathrm{MHz}, \mathrm{CDCl}_{3}\right)$ 


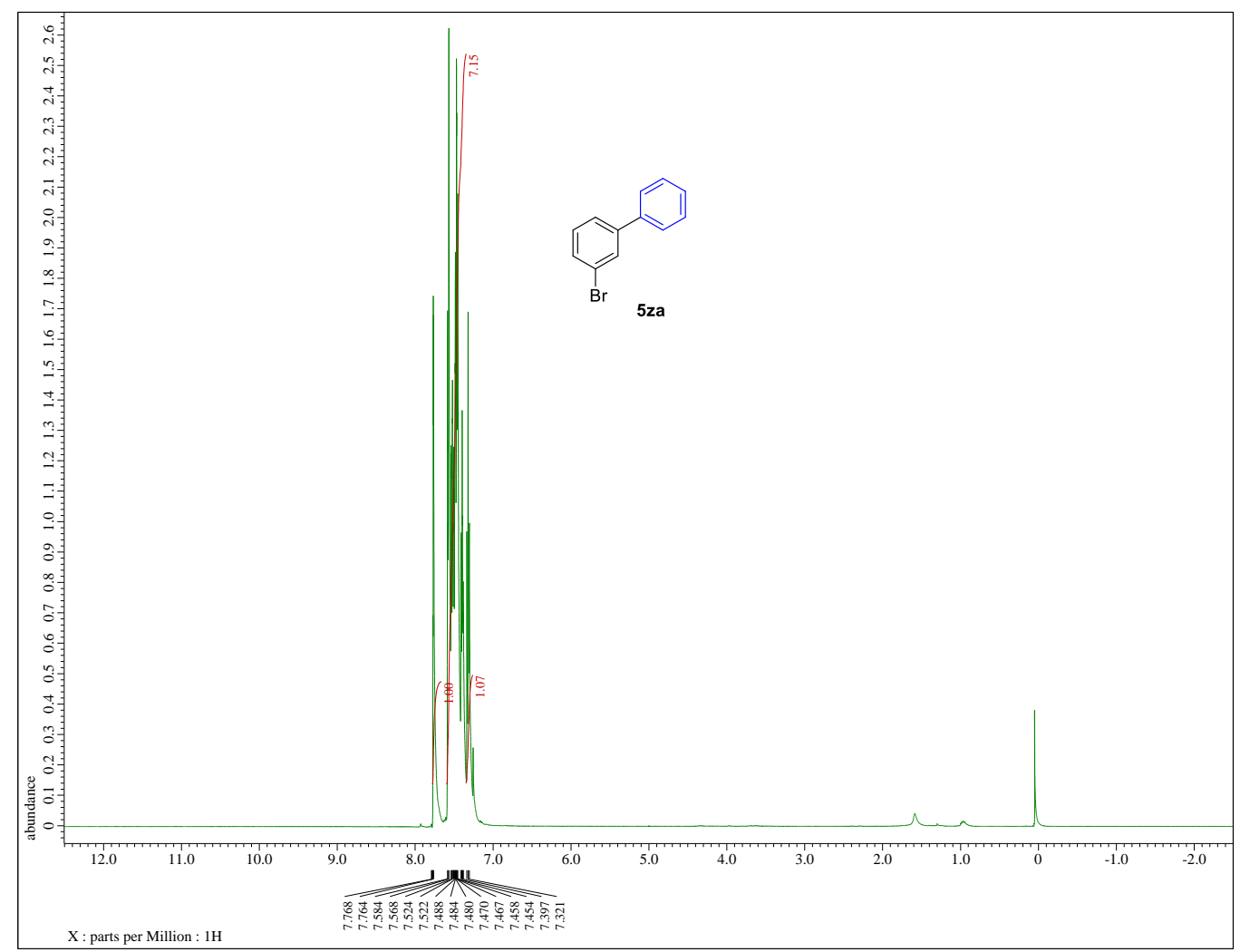

Figure S58-1. ${ }^{1} \mathrm{H}$ NMR spectrum of compound $5 z a\left(500 \mathrm{MHz}, \mathrm{CDCl}_{3}\right)$

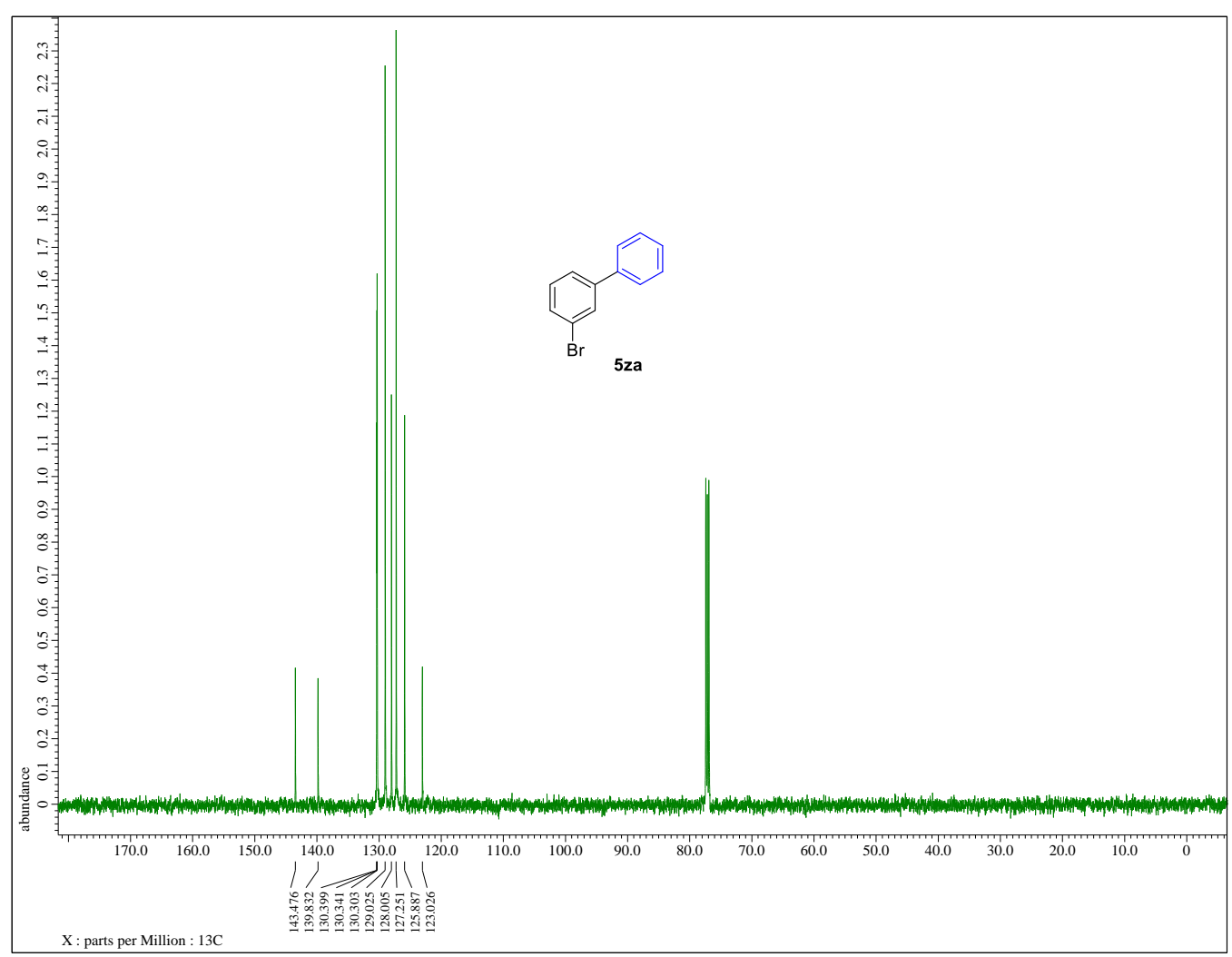

Figure S58-2. ${ }^{13} \mathrm{C}$ NMR spectrum of compound $5 z a\left(125 \mathrm{MHz}, \mathrm{CDCl}_{3}\right)$ 


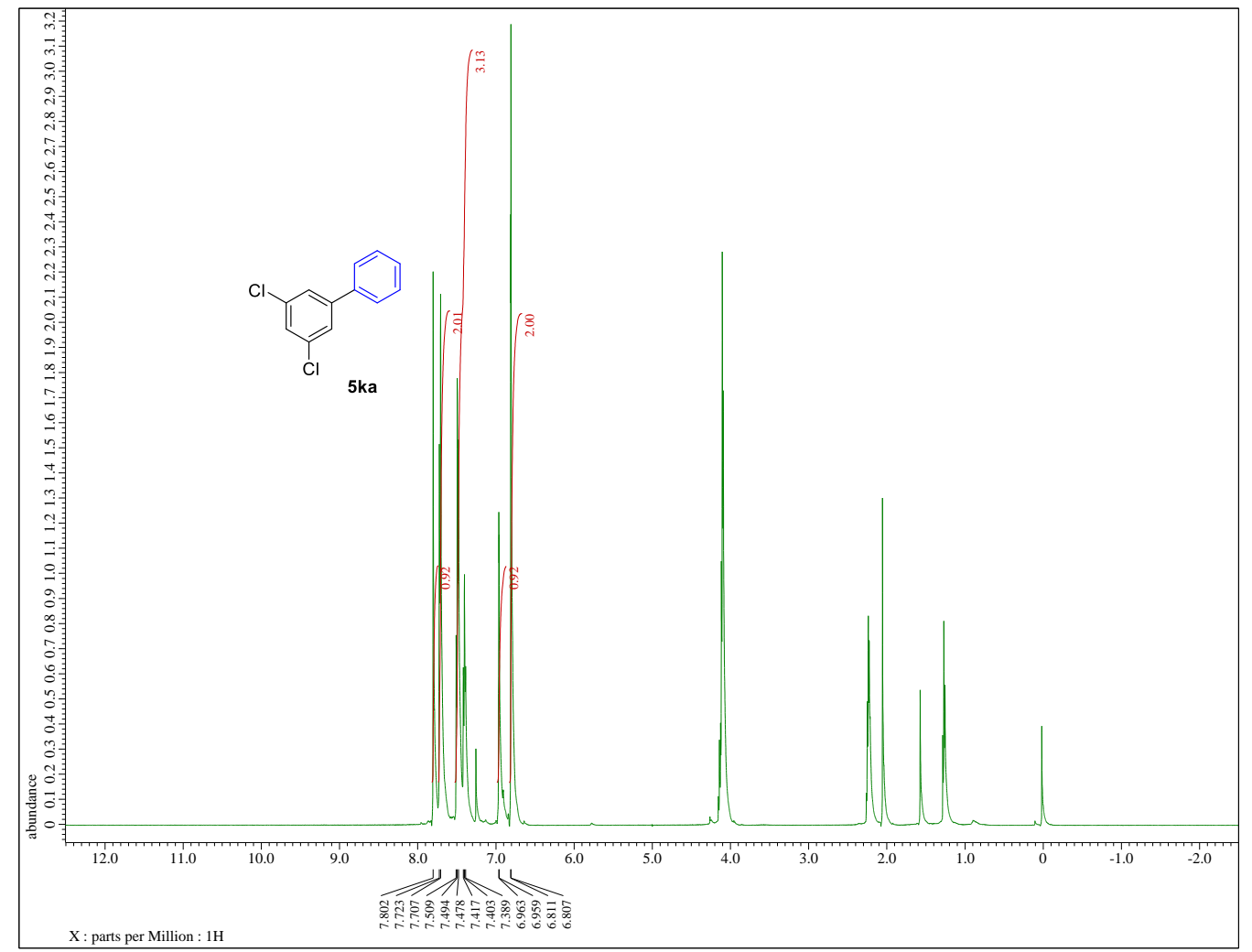

Figure S59-1. ${ }^{1} \mathrm{H}$ NMR spectrum of compound $\mathbf{5 k a}\left(500 \mathrm{MHz}, \mathrm{CDCl}_{3}\right)$

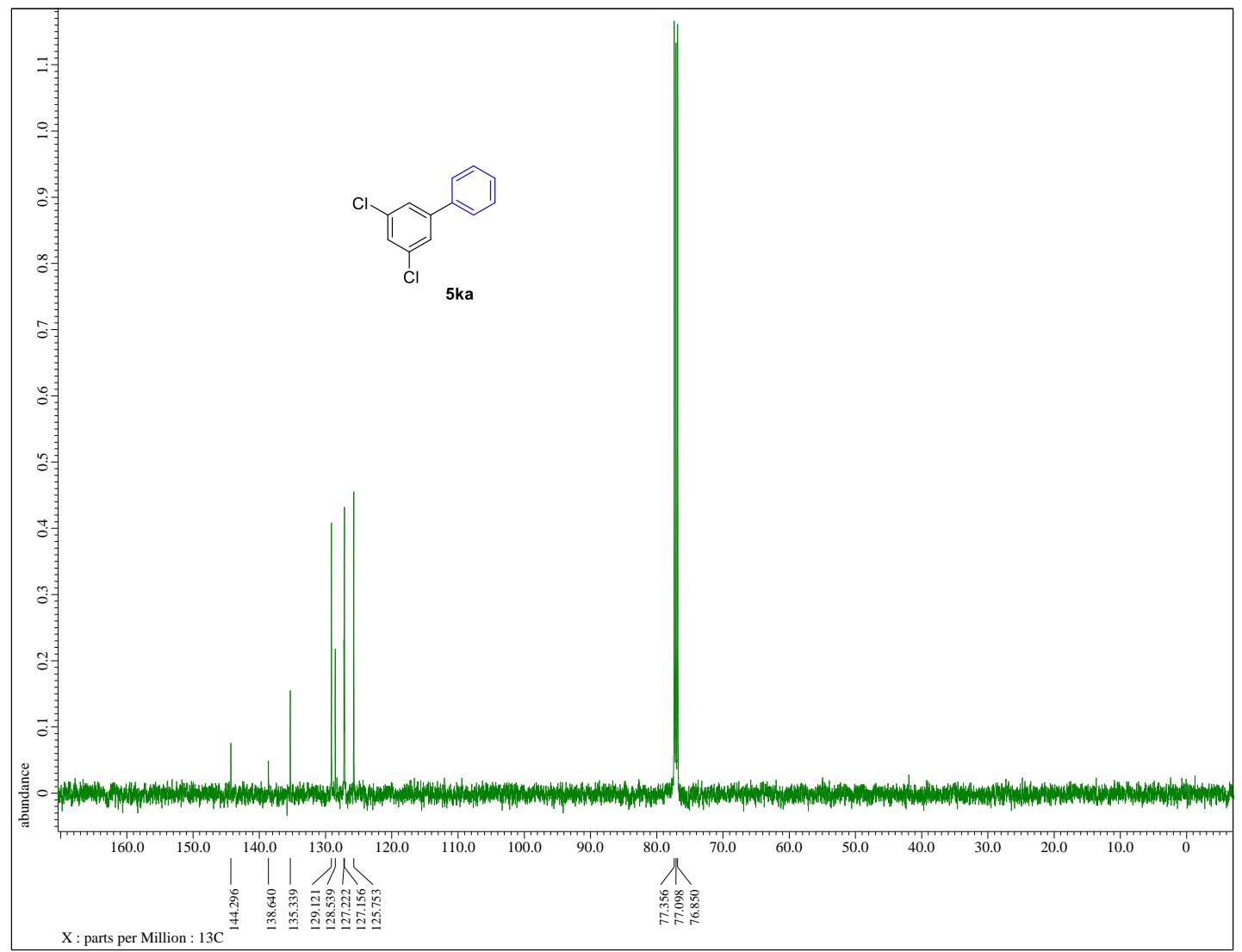

Figure S59-2. ${ }^{13} \mathrm{C}$ NMR spectrum of compound 5ka $\left(125 \mathrm{MHz}, \mathrm{CDCl}_{3}\right)$ 


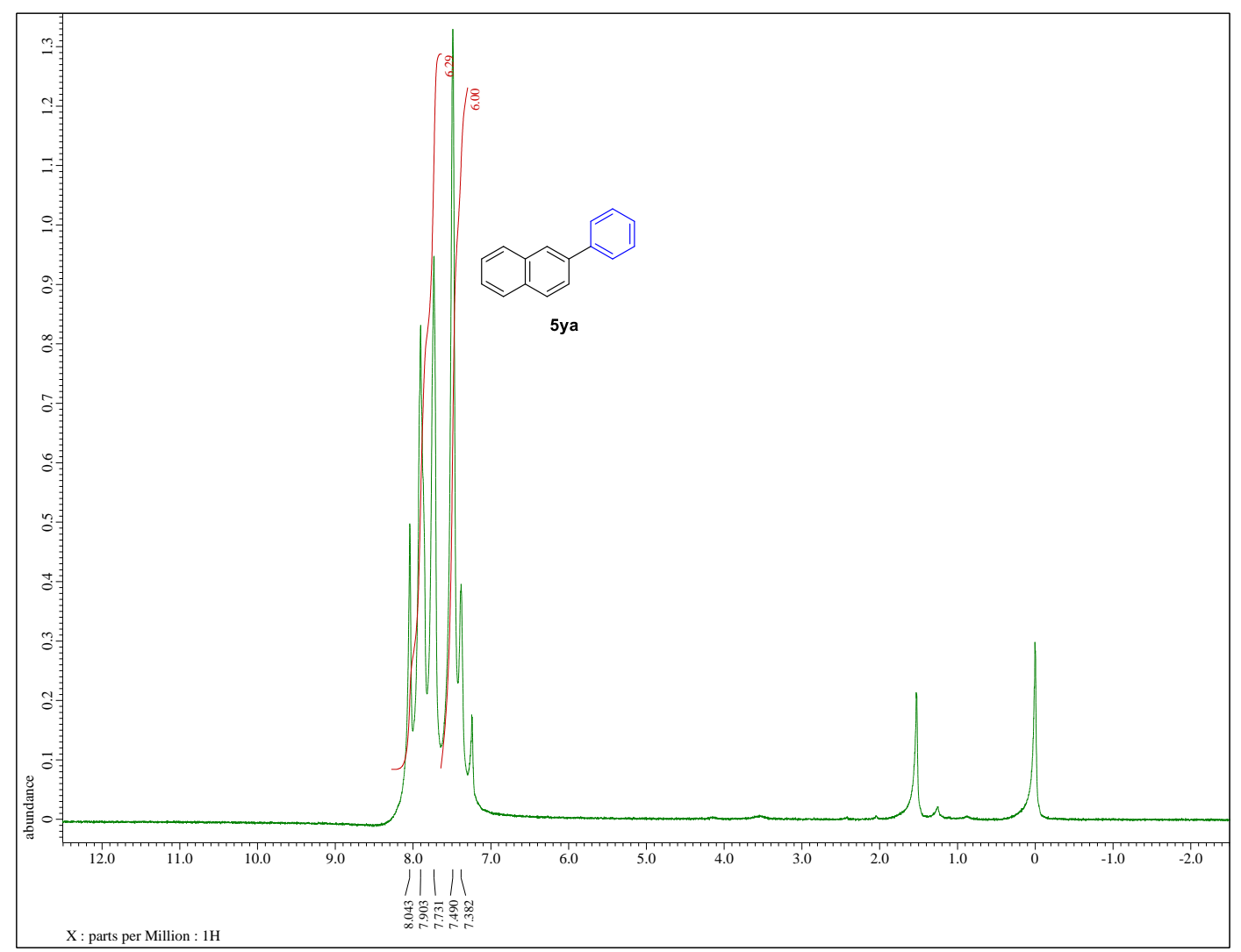

Figure S60-1. ${ }^{1} \mathrm{H}$ NMR spectrum of compound $\mathbf{5 y a}\left(500 \mathrm{MHz}, \mathrm{CDCl}_{3}\right)$

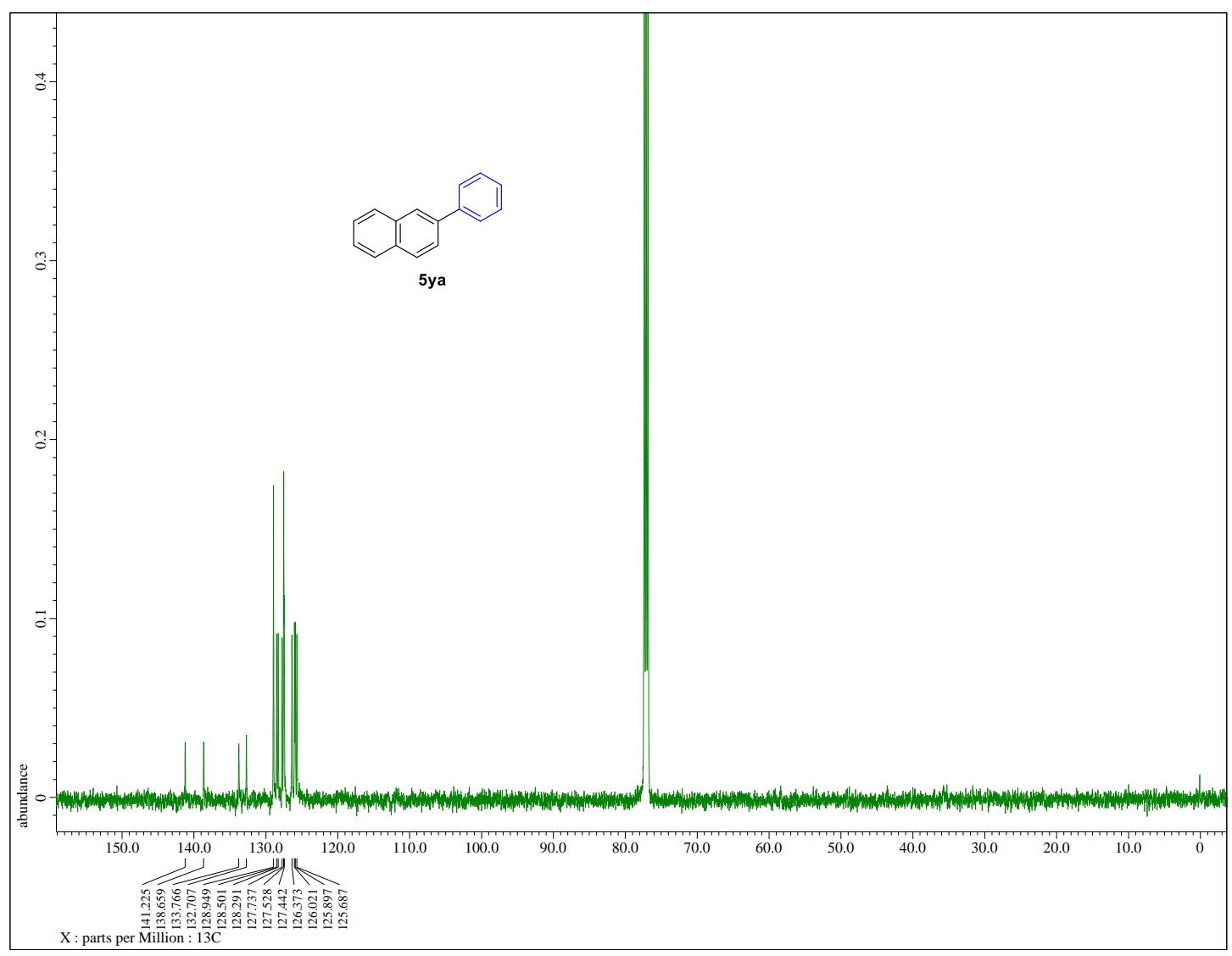

Figure S60-2. ${ }^{13} \mathrm{C}$ NMR spectrum of compound 5 ya $\left(125 \mathrm{MHz}, \mathrm{CDCl}_{3}\right)$ 


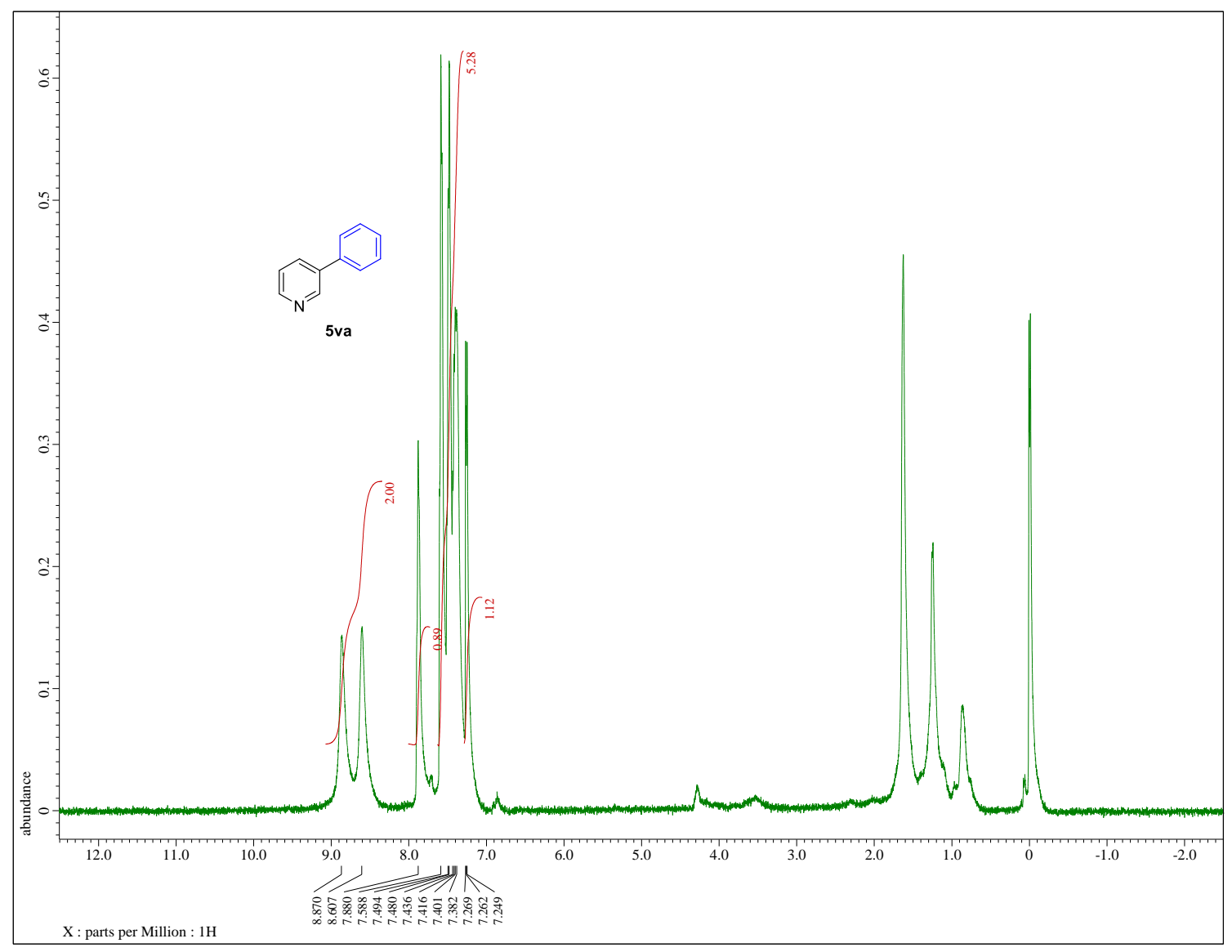

Figure S61-1. ${ }^{1} \mathrm{H}$ NMR spectrum of compound $5 v a\left(500 \mathrm{MHz}, \mathrm{CDCl}_{3}\right)$

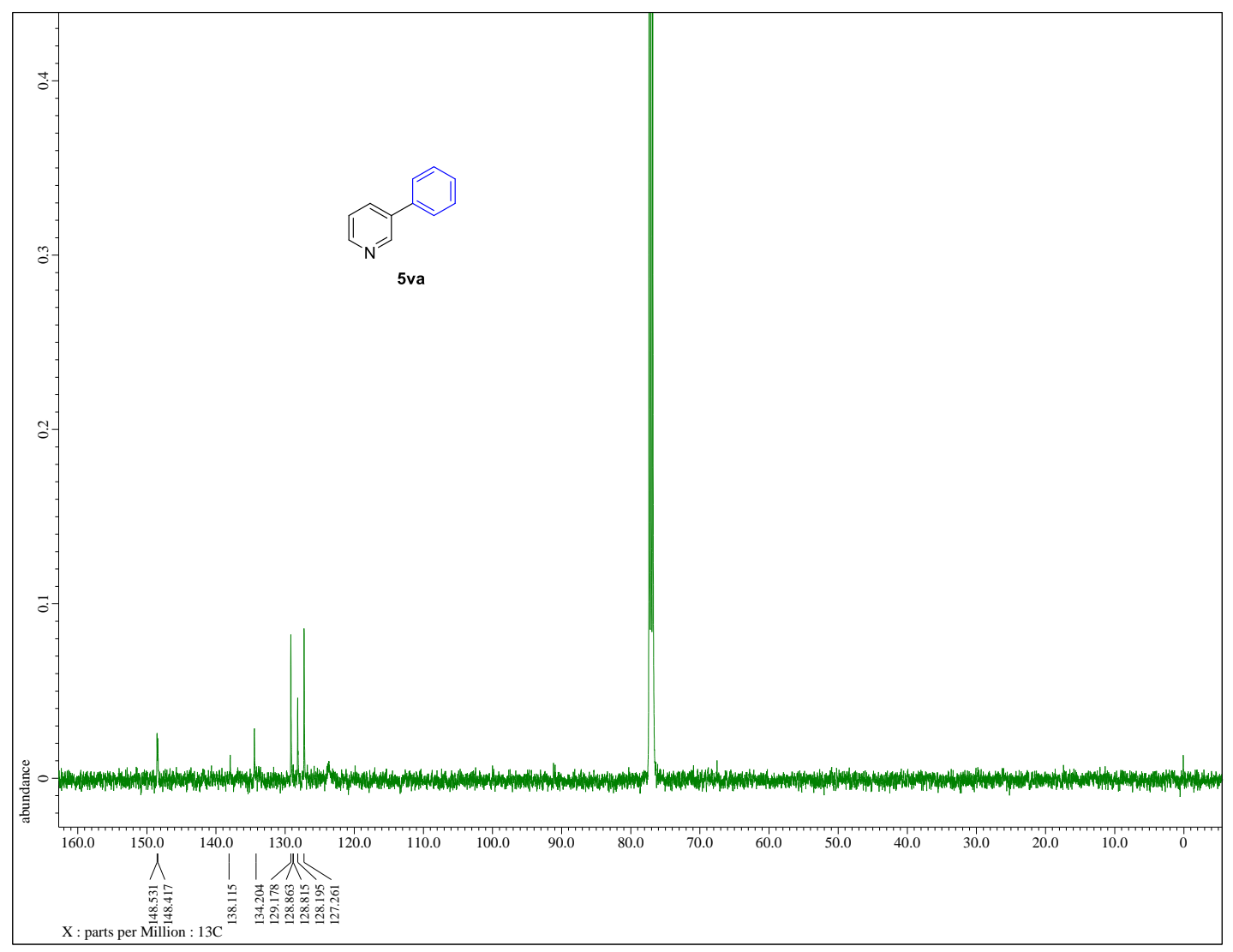

Figure S61-2. ${ }^{13} \mathrm{C}$ NMR spectrum of compound $5 v a\left(125 \mathrm{MHz}, \mathrm{CDCl}_{3}\right)$ 


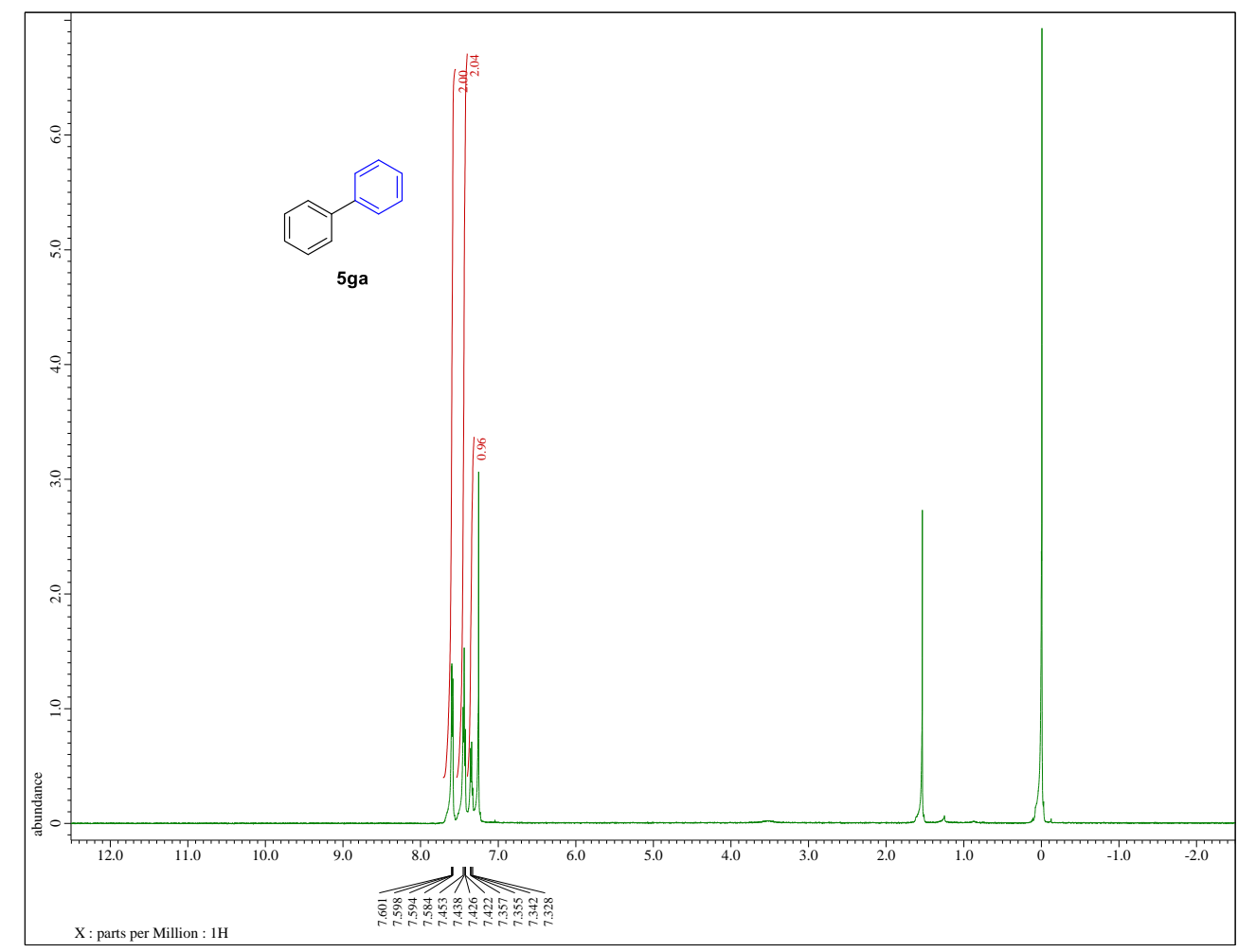

Figure S62-1. ${ }^{1} \mathrm{H}$ NMR spectrum of compound $5 g a\left(500 \mathrm{MHz}, \mathrm{CDCl}_{3}\right)$

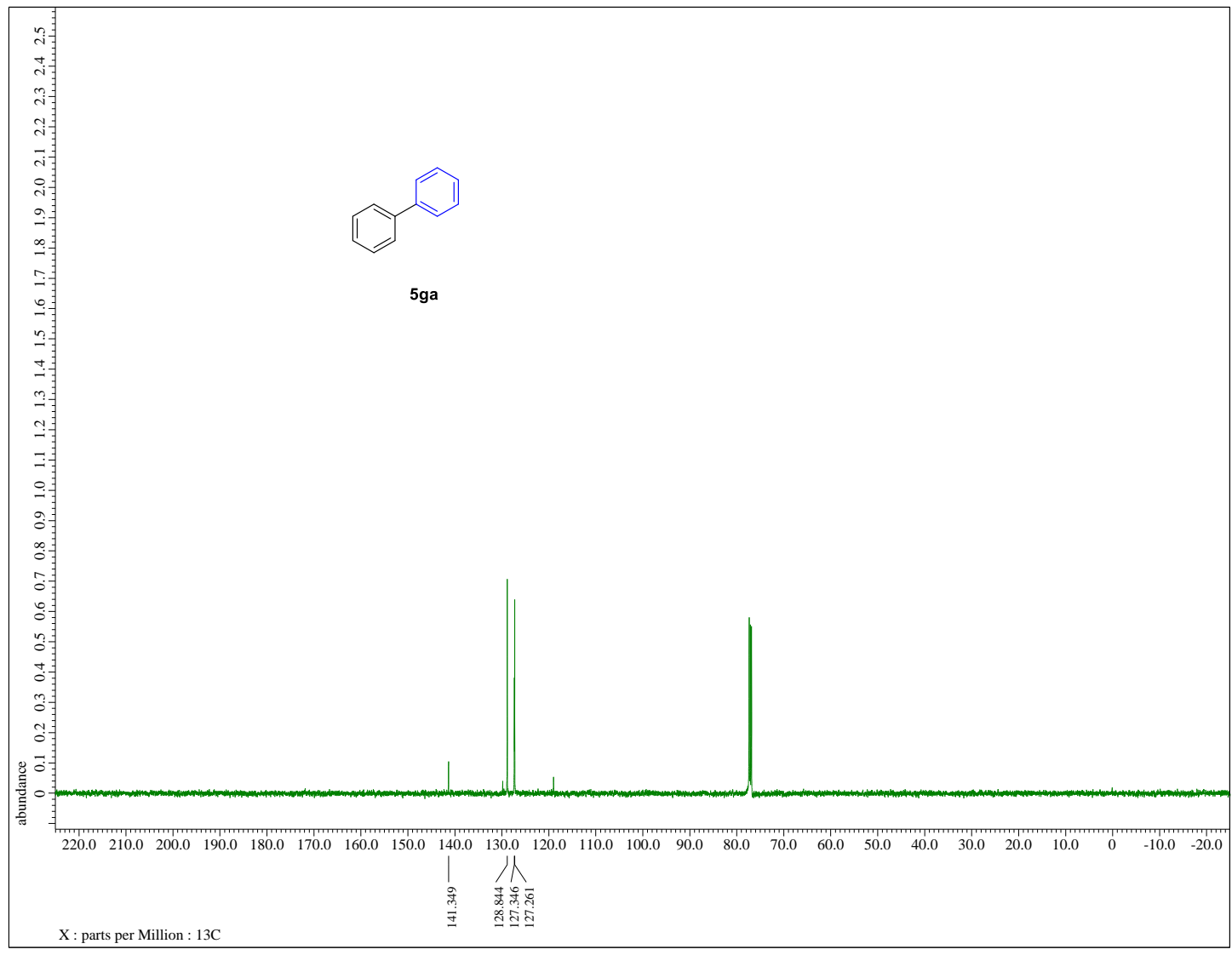

Figure S62-2. ${ }^{13} \mathrm{C}$ NMR spectrum of compound $5 g a\left(125 \mathrm{MHz}, \mathrm{CDCl}_{3}\right)$ 


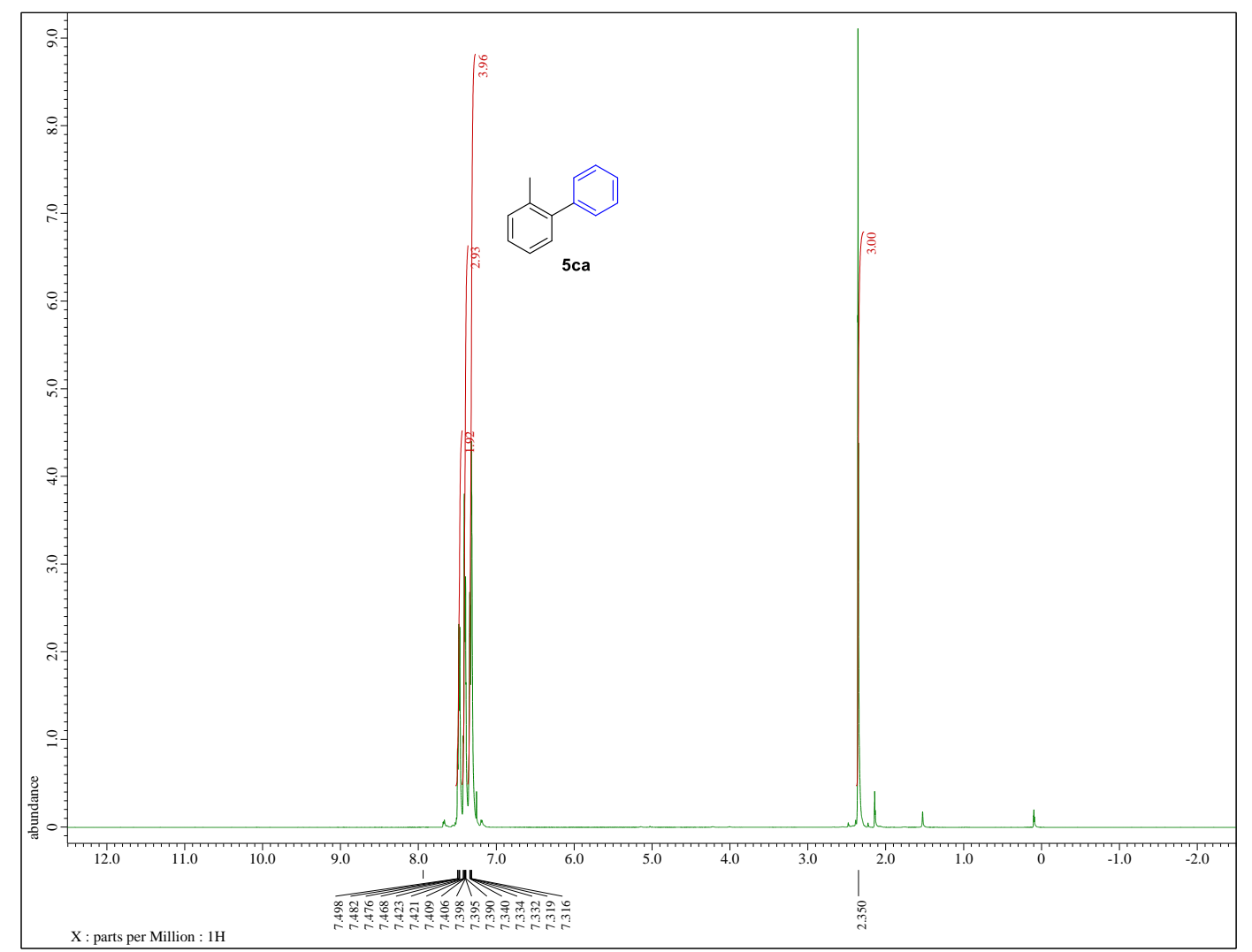

Figure S63-1. ${ }^{1} \mathrm{H}$ NMR spectrum of compound $\mathbf{5 c a}\left(500 \mathrm{MHz}, \mathrm{CDCl}_{3}\right)$

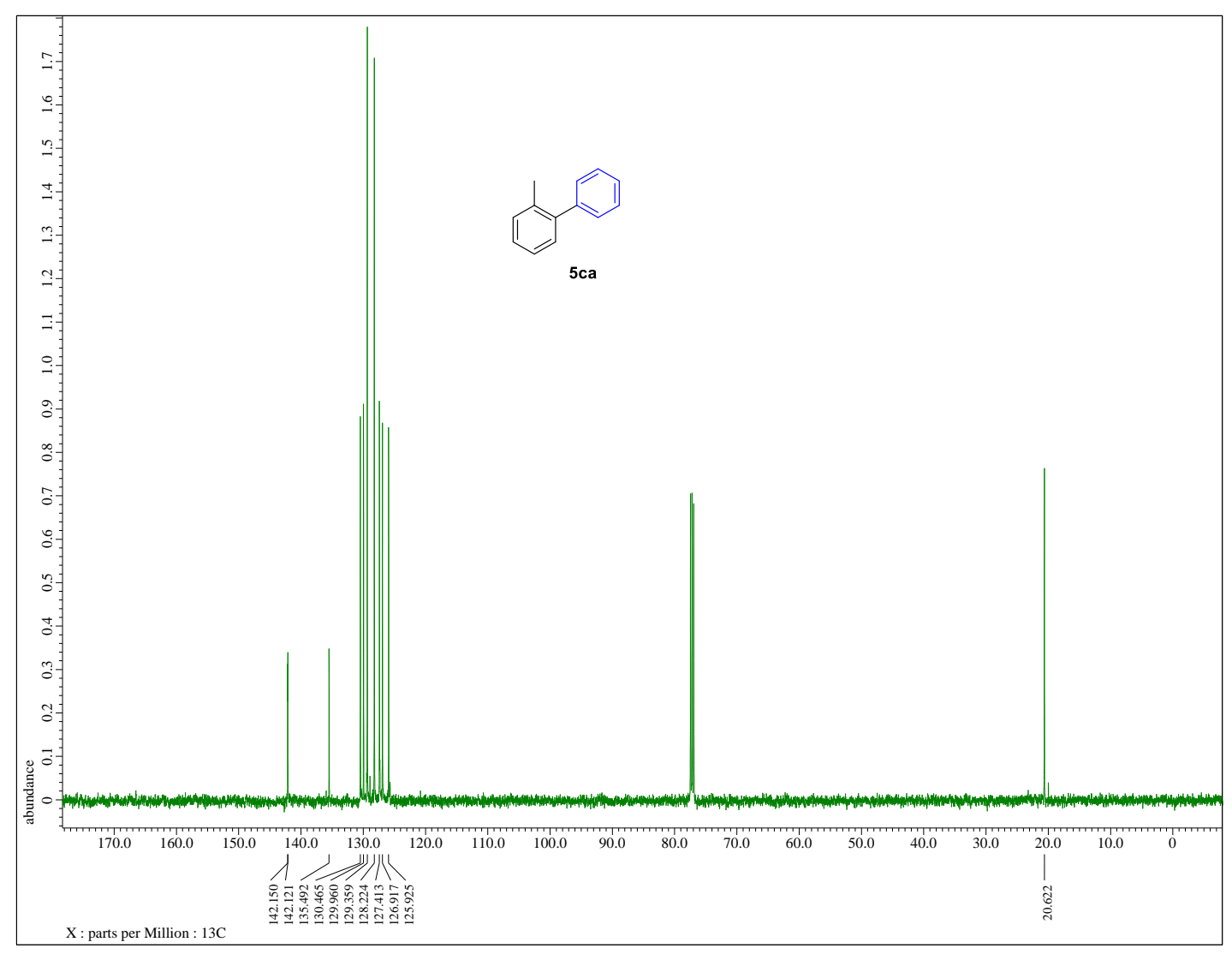

Figure S63-2. ${ }^{13} \mathrm{C}$ NMR spectrum of compound 5 ca $\left(125 \mathrm{MHz}, \mathrm{CDCl}_{3}\right)$ 


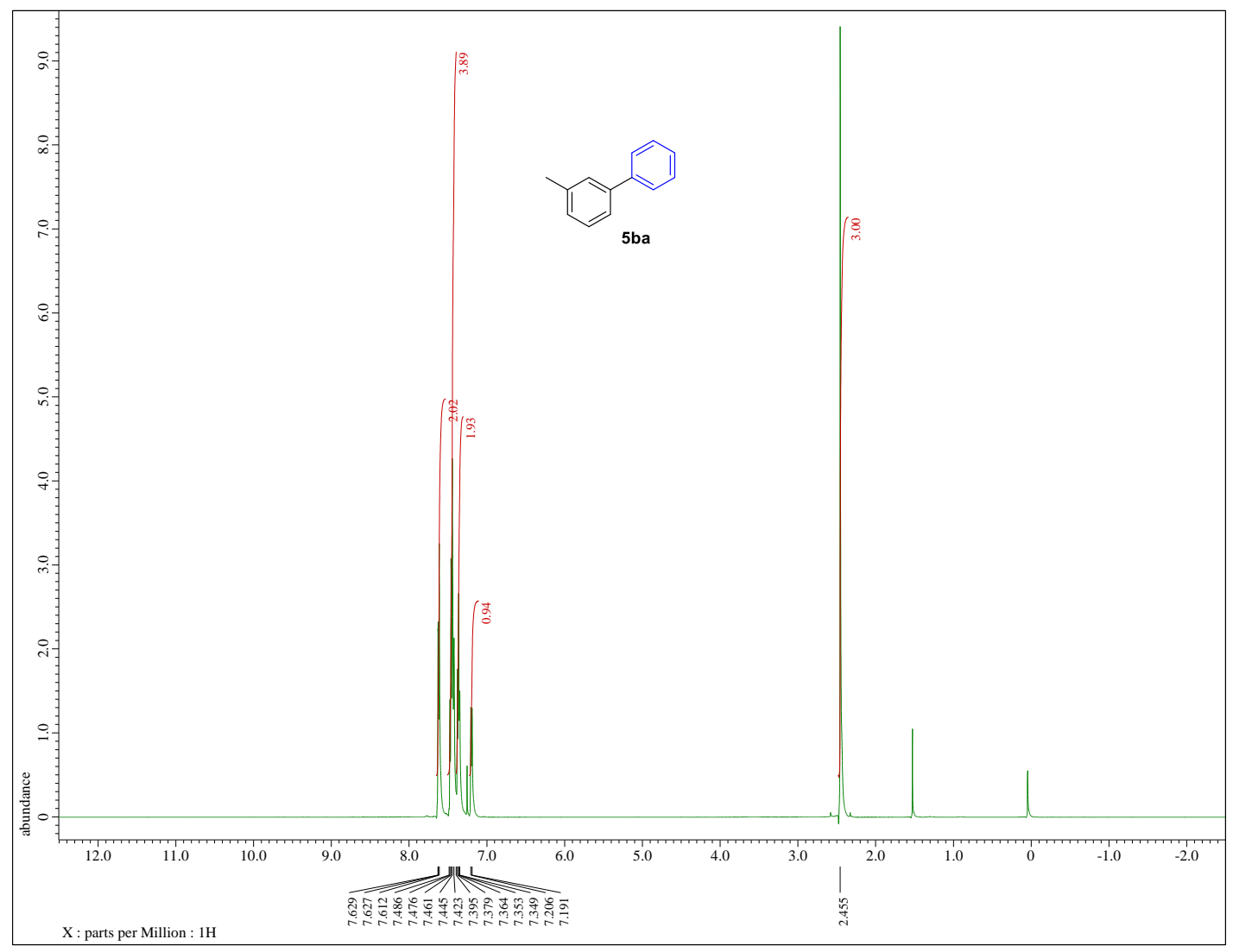

Figure S64-1. ${ }^{1} \mathrm{H}$ NMR spectrum of compound $5 \mathbf{b a}\left(500 \mathrm{MHz}, \mathrm{CDCl}_{3}\right)$

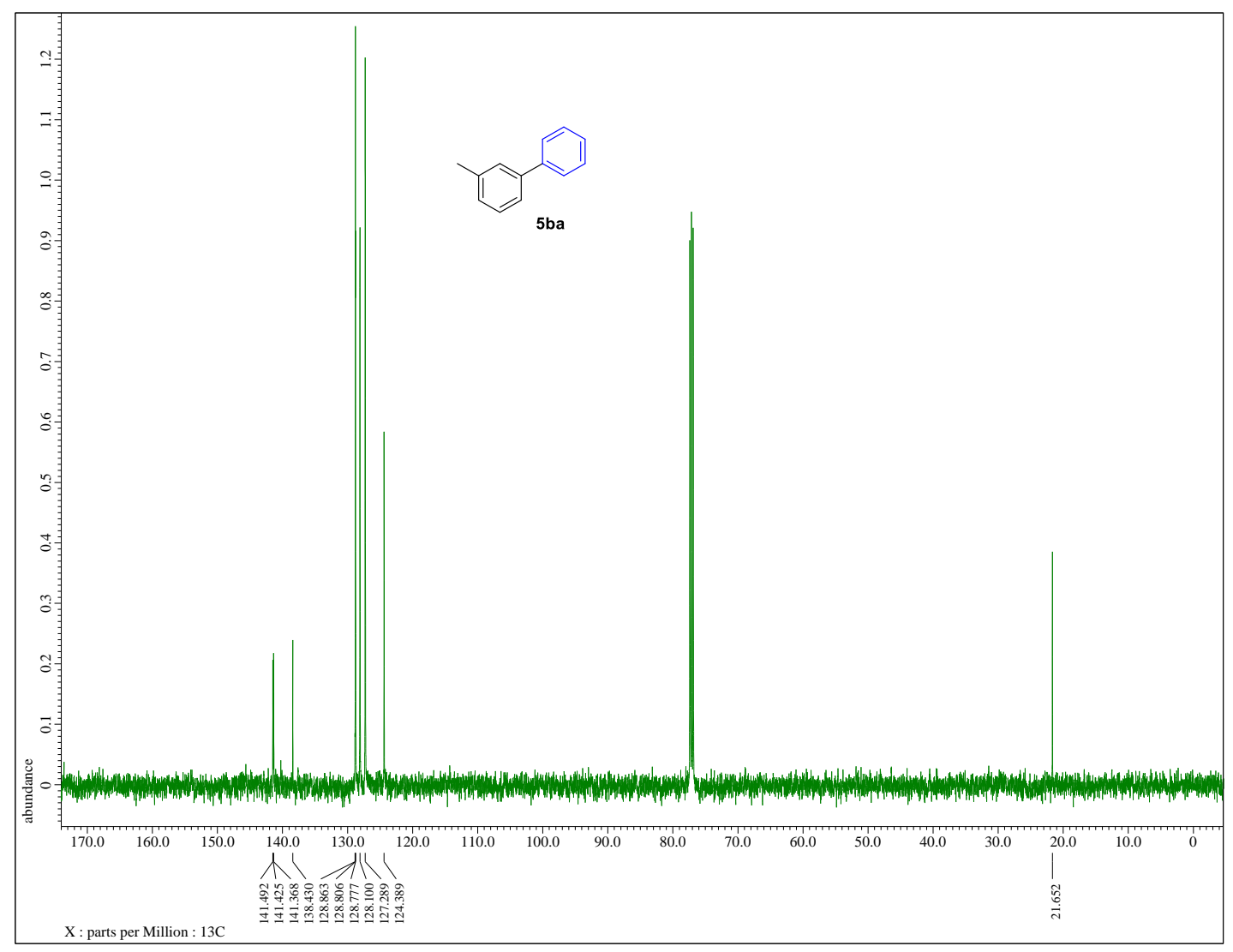

Figure S64-2. ${ }^{13} \mathrm{C}$ NMR spectrum of compound $5 \mathbf{b a}\left(125 \mathrm{MHz}, \mathrm{CDCl}_{3}\right)$ 


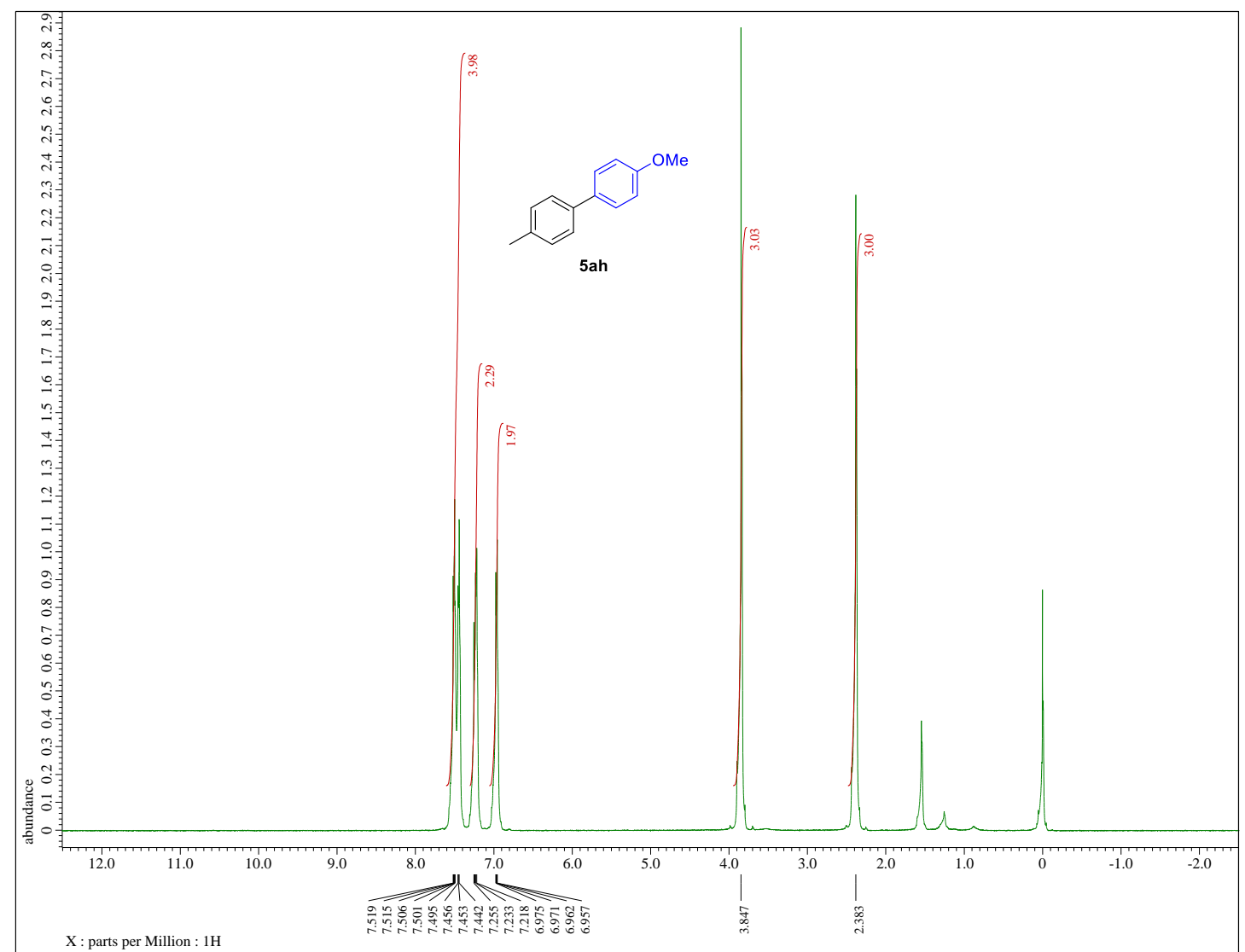

Figure S65-1. ${ }^{1} \mathrm{H}$ NMR spectrum of compound $\mathbf{5 a h}\left(500 \mathrm{MHz}, \mathrm{CDCl}_{3}\right)$

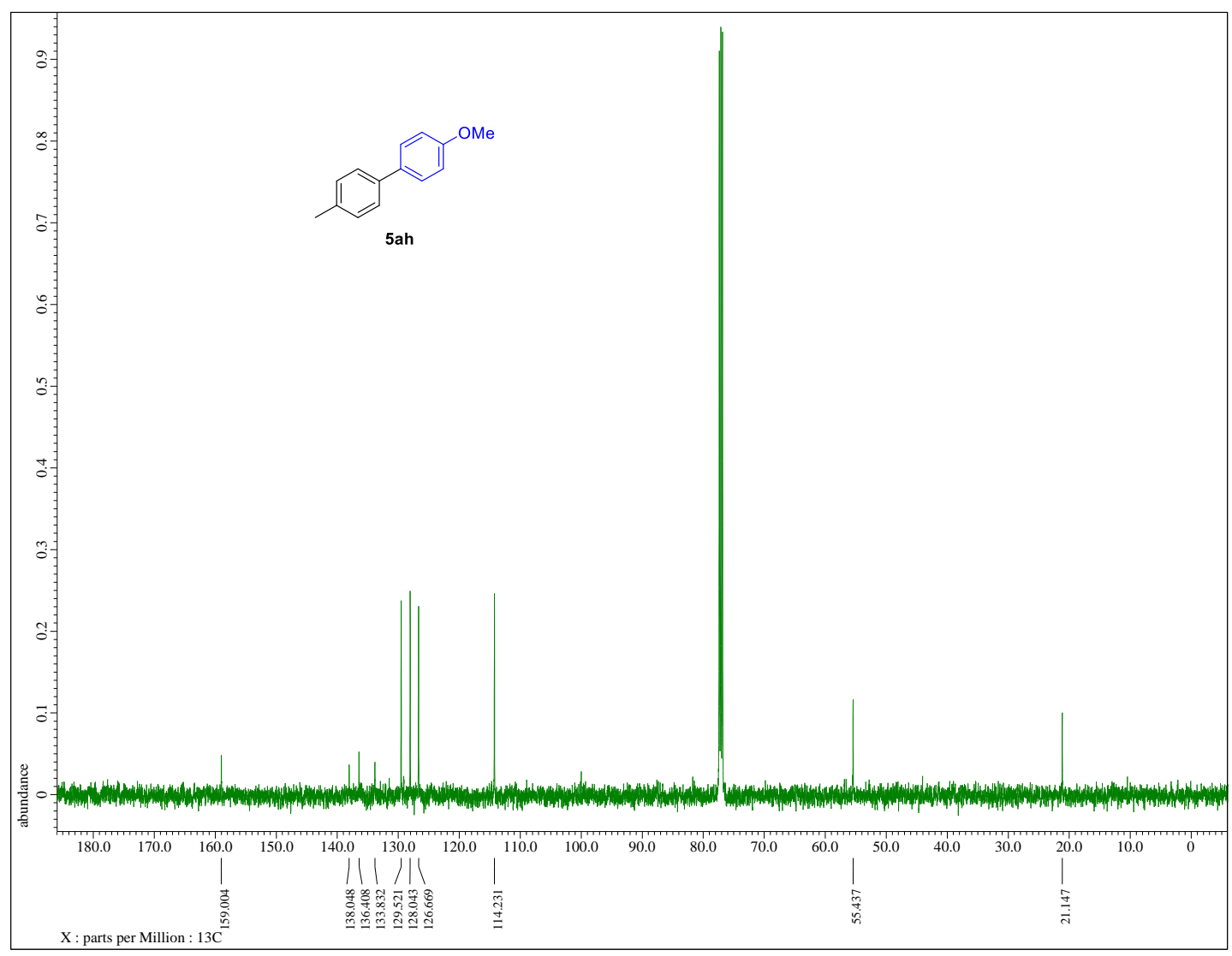

Figure S65-2. ${ }^{13} \mathrm{C}$ NMR spectrum of compound $\mathbf{5 a h}\left(125 \mathrm{MHz}, \mathrm{CDCl}_{3}\right)$ 


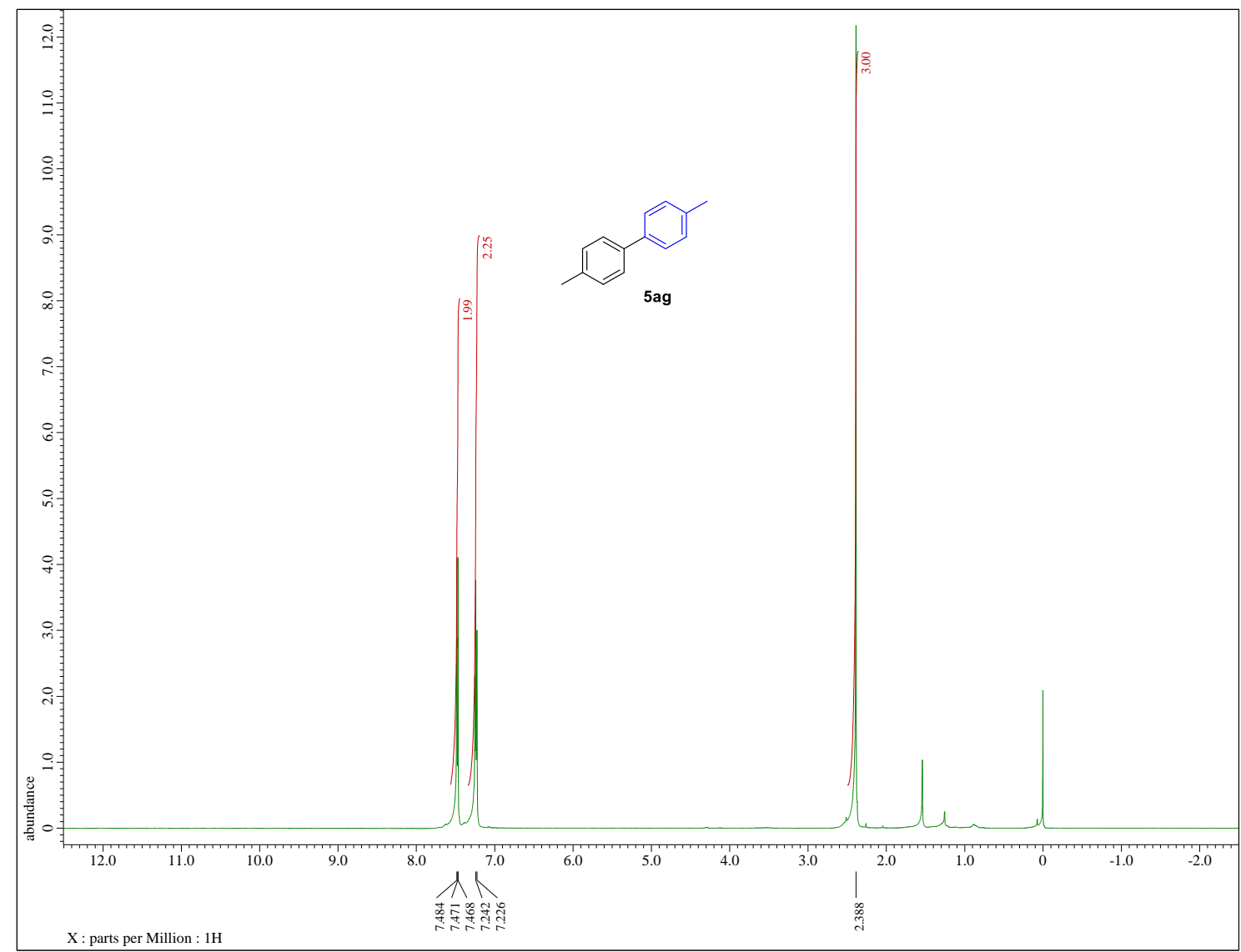

Figure S66-1. ${ }^{1} \mathrm{H}$ NMR spectrum of compound $\mathbf{5 a g}\left(500 \mathrm{MHz}, \mathrm{CDCl}_{3}\right)$

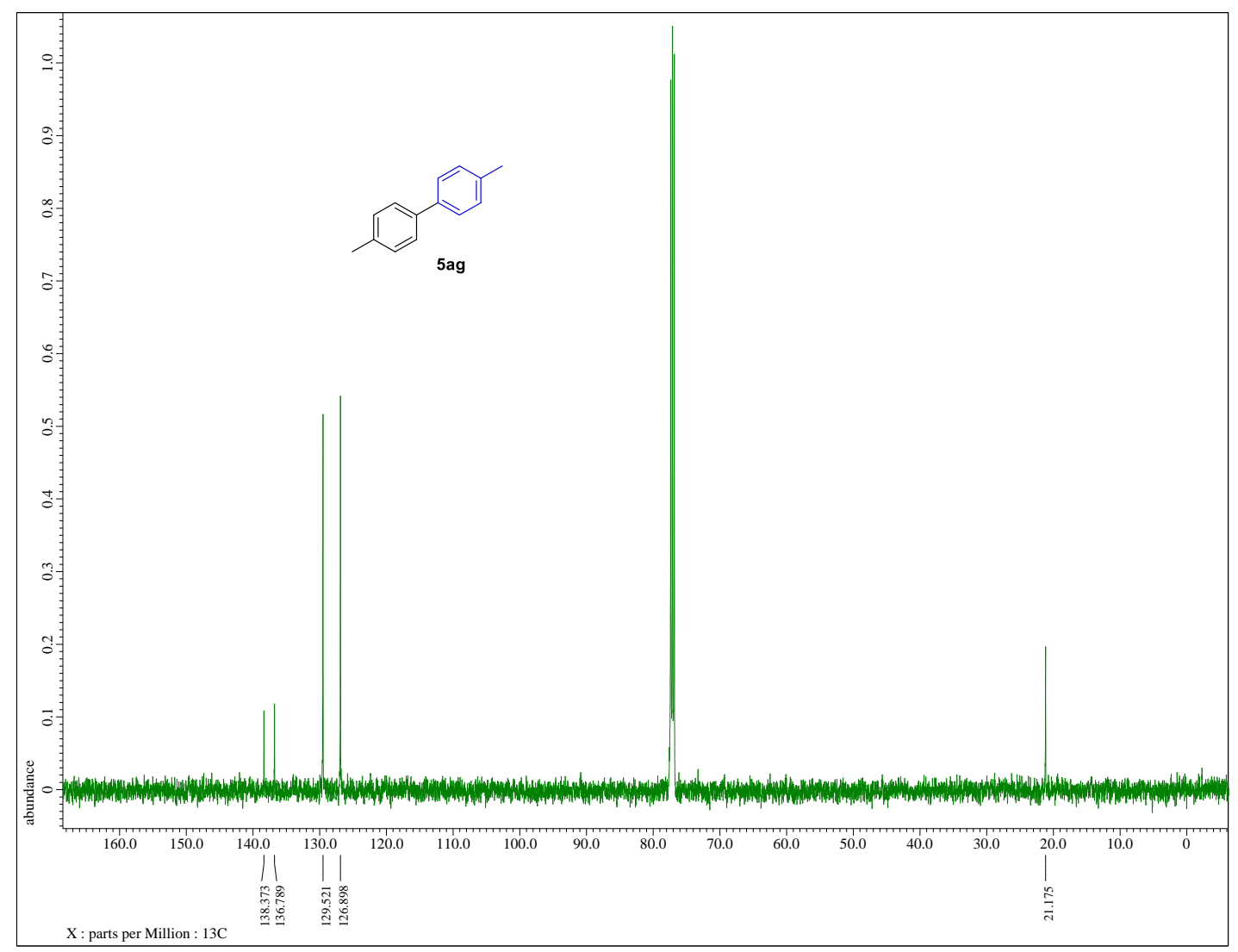

Figure S66-2. ${ }^{13} \mathrm{C}$ NMR spectrum of compound $\mathbf{5 a g}\left(125 \mathrm{MHz}, \mathrm{CDCl}_{3}\right)$ 


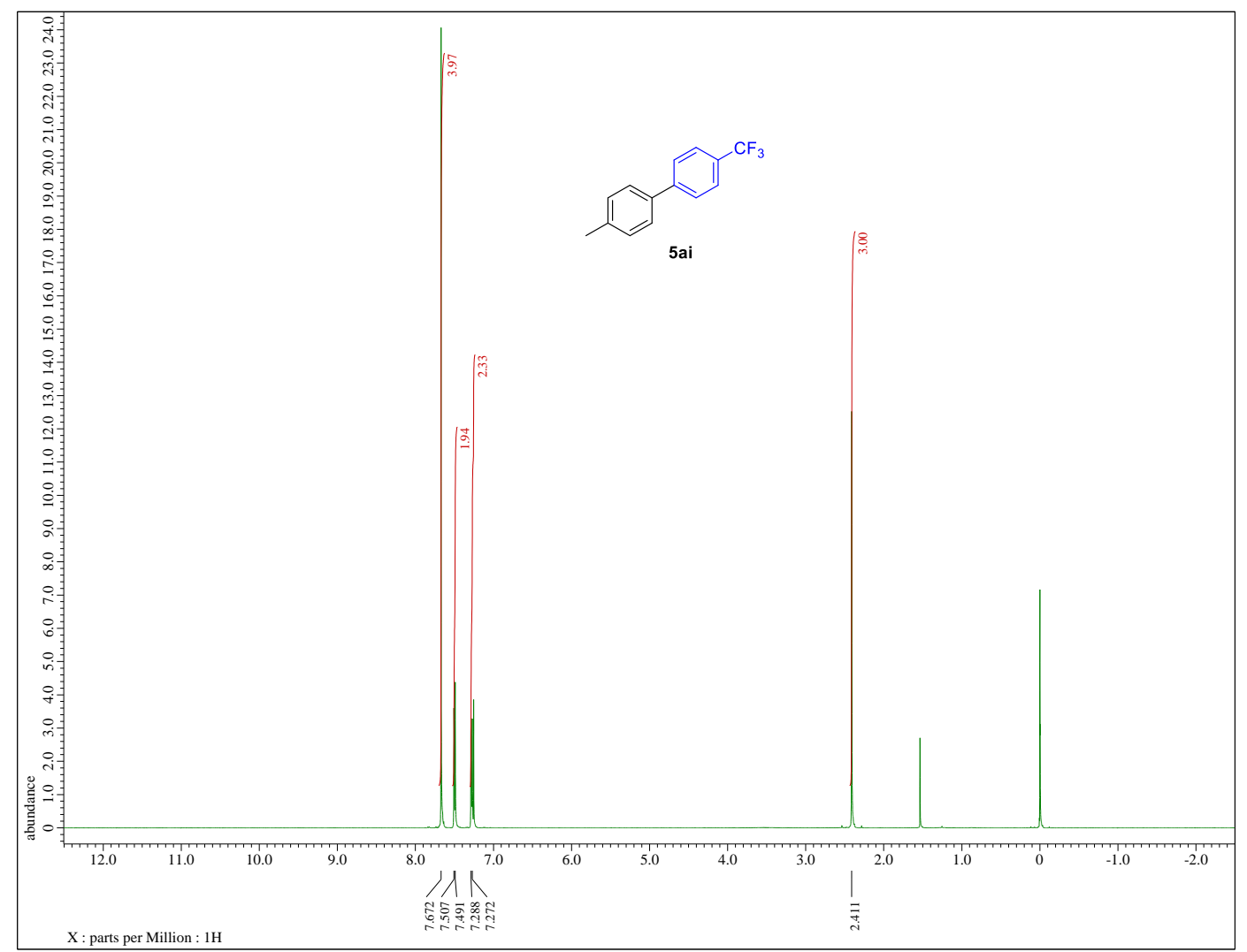

Figure S67-1. ${ }^{1} \mathrm{H}$ NMR spectrum of compound 5 ai $\left(500 \mathrm{MHz}, \mathrm{CDCl}_{3}\right)$

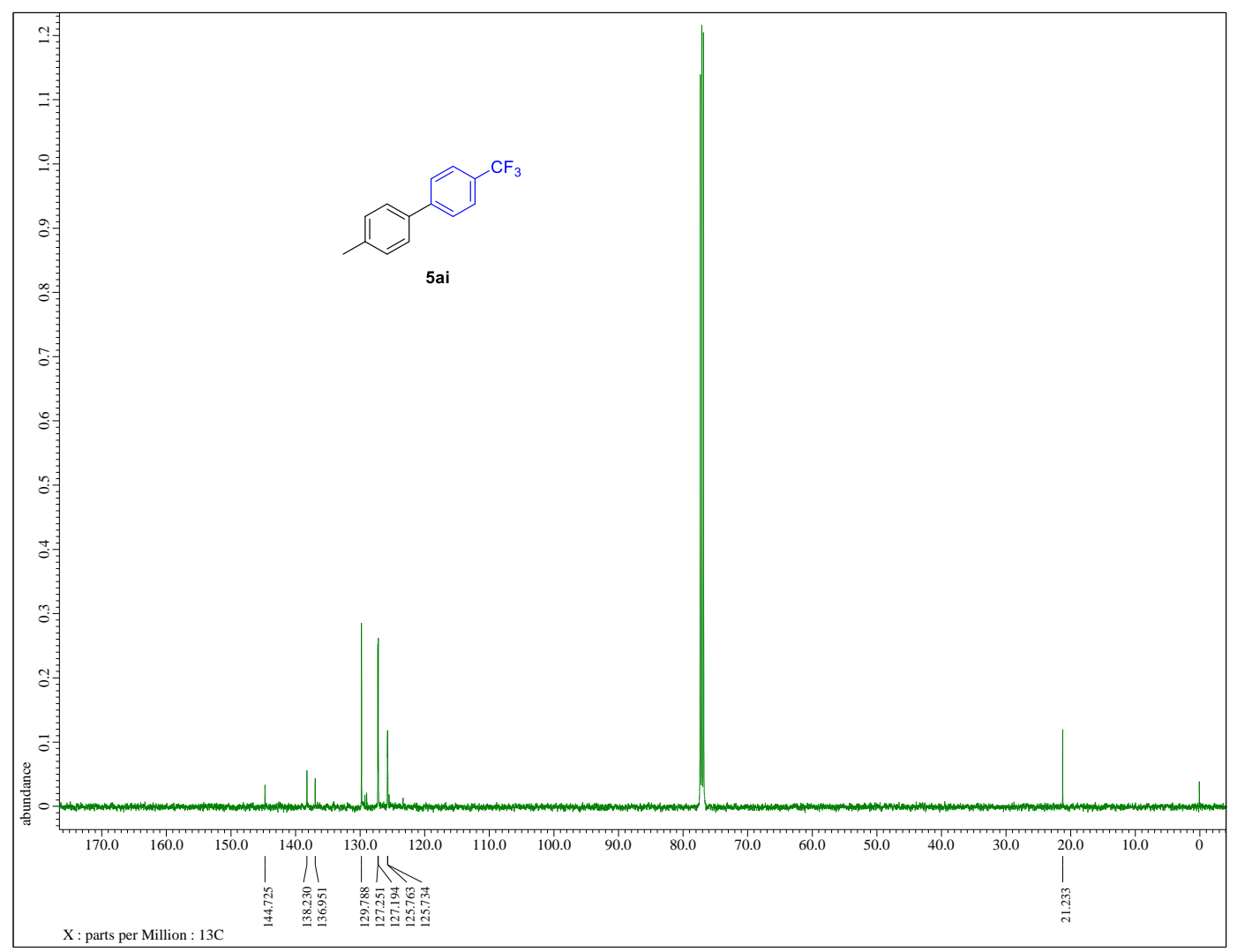

Figure $\mathbf{S 6 7 - 2} .{ }^{13} \mathrm{C} \mathrm{NMR}$ spectrum of compound 5 ai $\left(125 \mathrm{MHz}, \mathrm{CDCl}_{3}\right)$ 


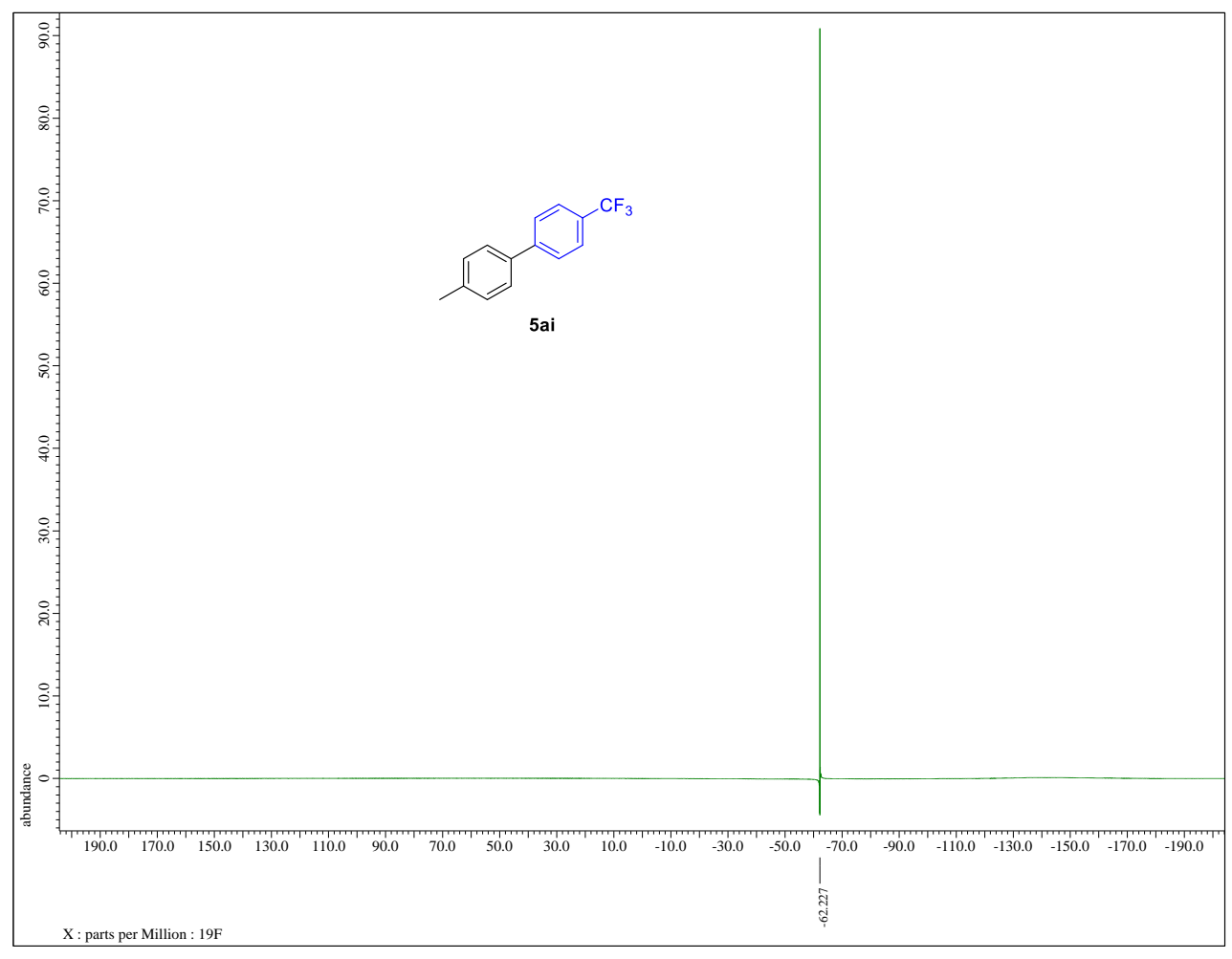

Figure S67-3. ${ }^{19} \mathrm{~F}$ NMR spectrum of compound 5ai $\left(470 \mathrm{MHz}, \mathrm{CDCl}_{3}\right)$ 


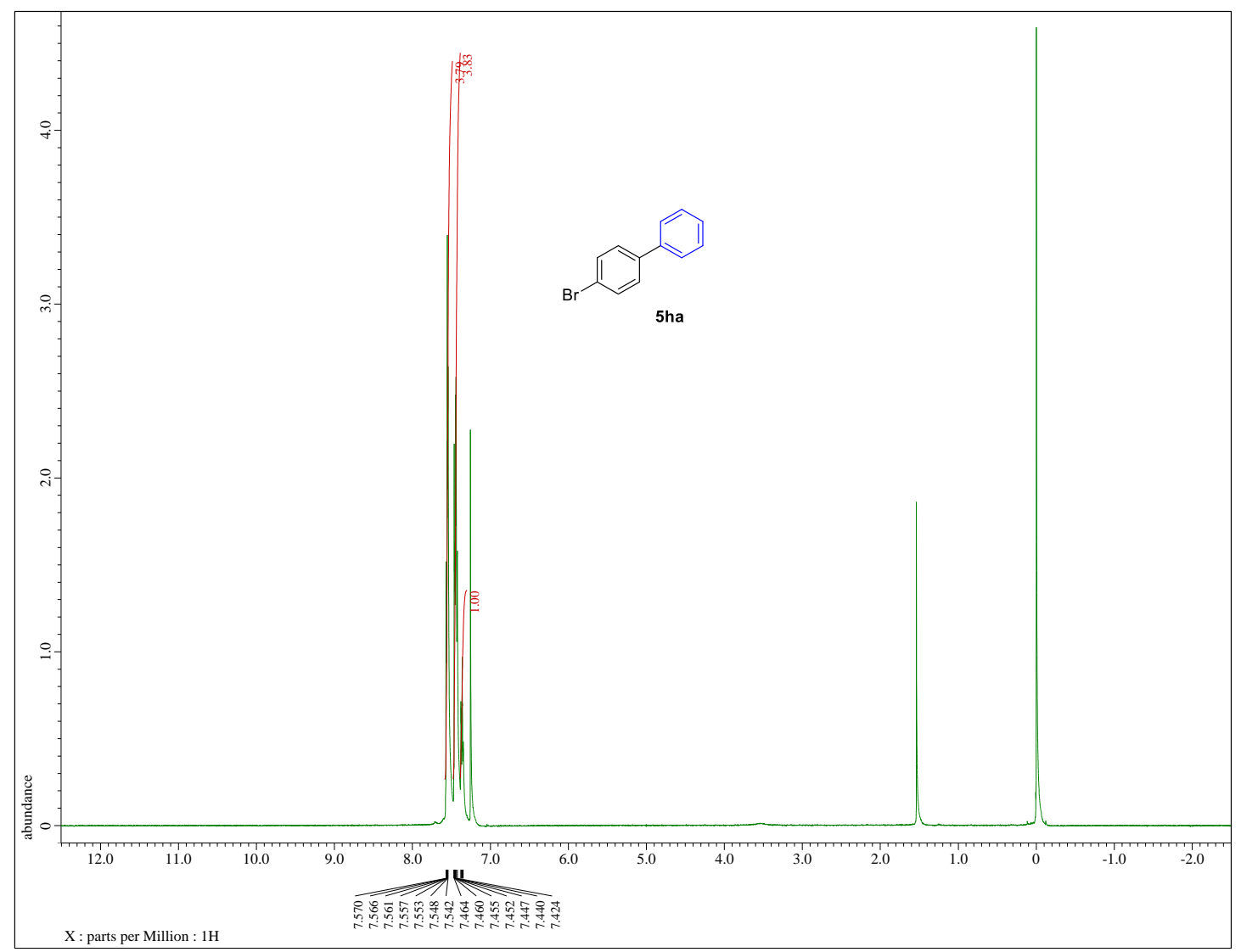

Figure S68-1. ${ }^{1} \mathrm{H}$ NMR spectrum of compound 5 ha $\left(500 \mathrm{MHz}, \mathrm{CDCl}_{3}\right)$

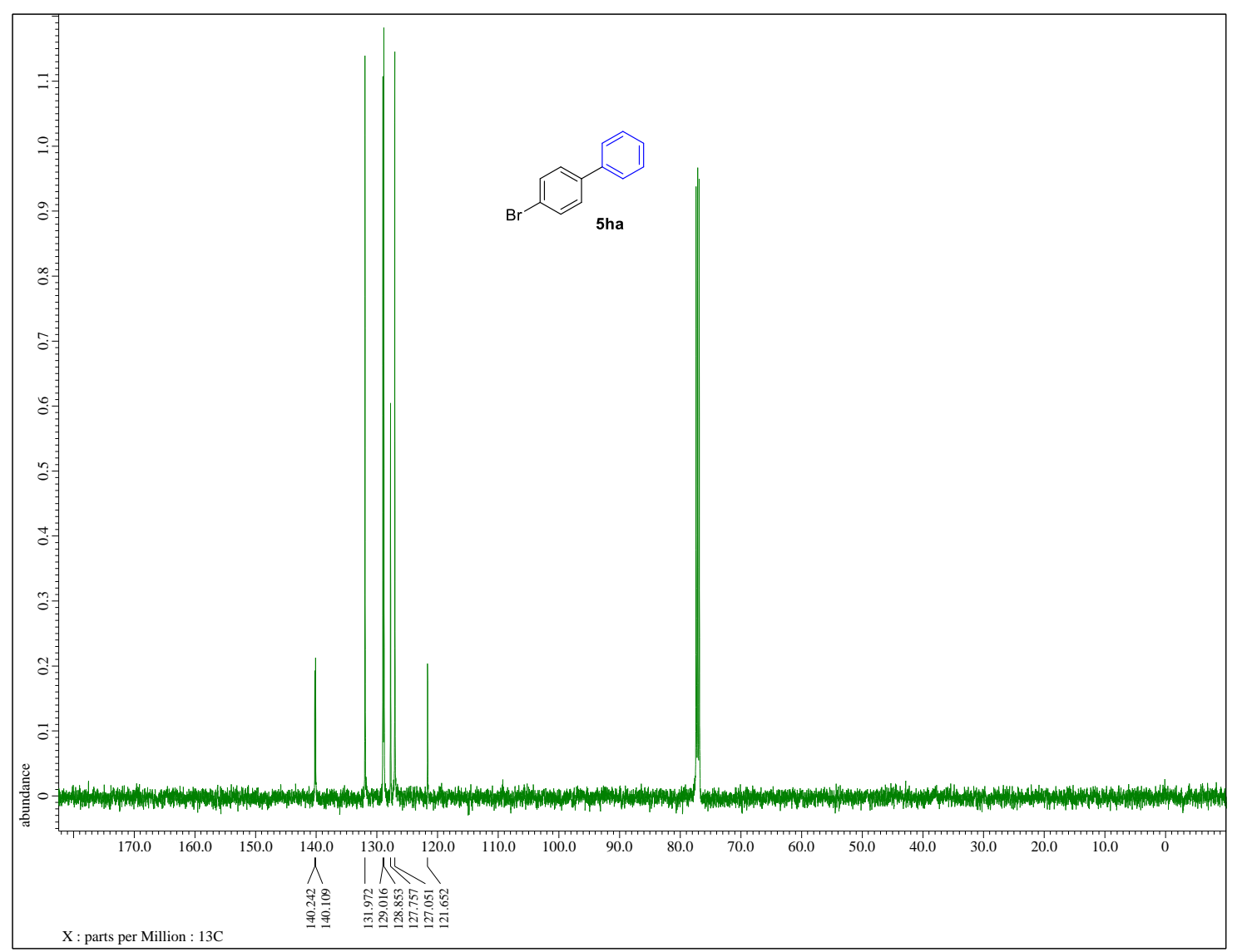

Figure S68-2. ${ }^{13} \mathrm{C}$ NMR spectrum of compound 5 ha $\left(125 \mathrm{MHz}, \mathrm{CDCl}_{3}\right)$ 


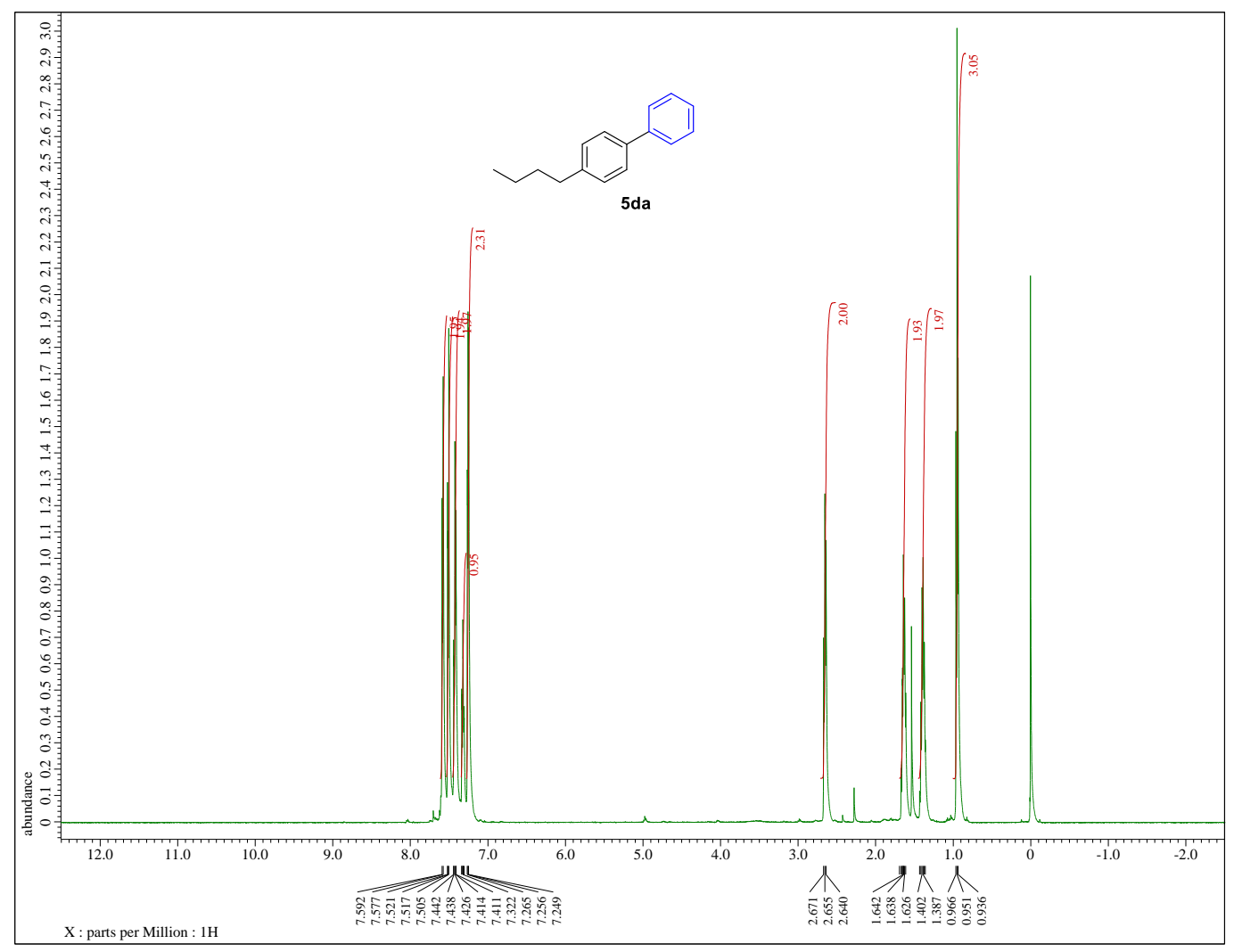

Figure S69-1. ${ }^{1} \mathrm{H}$ NMR spectrum of compound $5 \mathrm{da}\left(500 \mathrm{MHz}, \mathrm{CDCl}_{3}\right)$

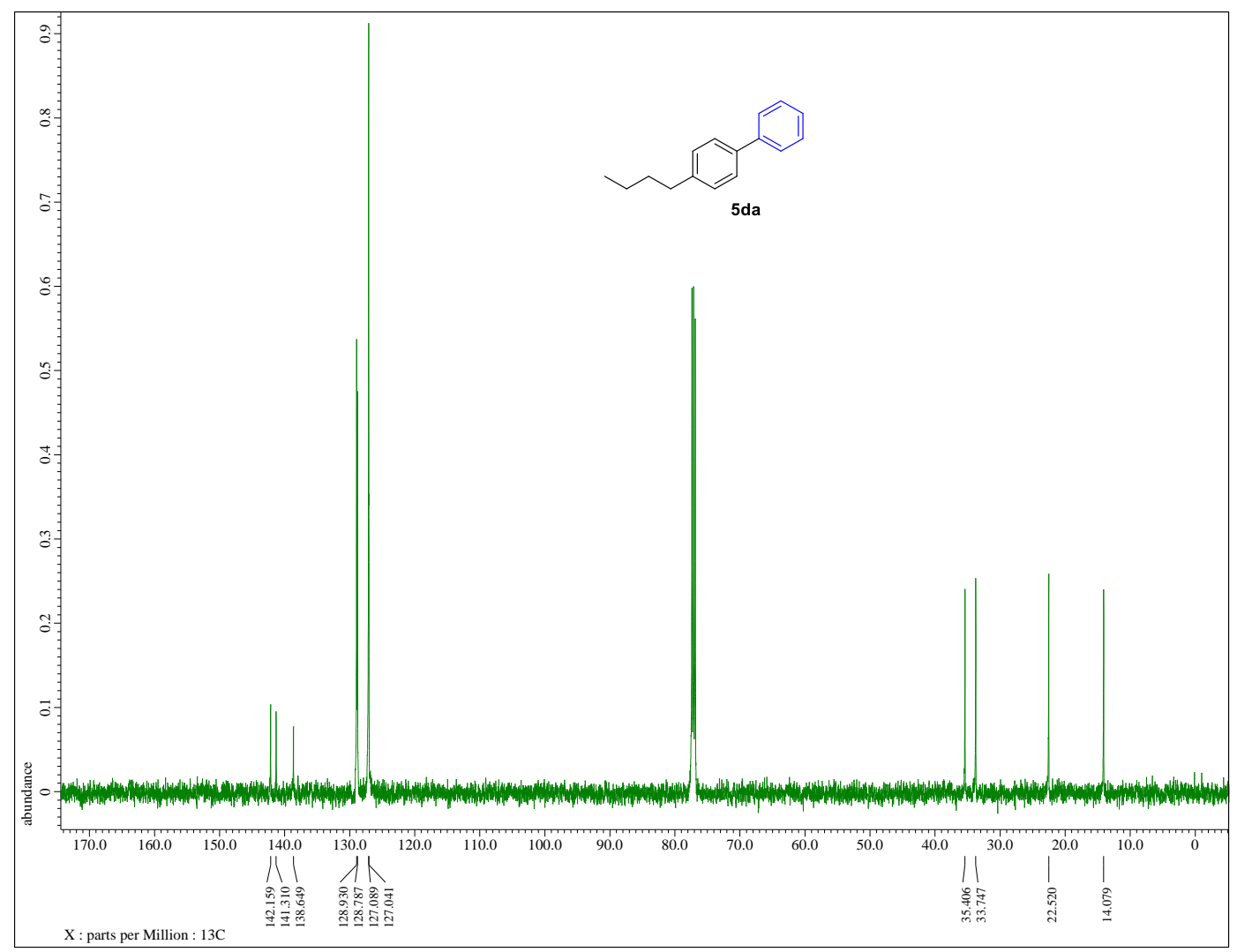

Figure S69-2. ${ }^{13} \mathrm{C}$ NMR spectrum of compound $5 \mathrm{da}\left(125 \mathrm{MHz}, \mathrm{CDCl}_{3}\right)$ 


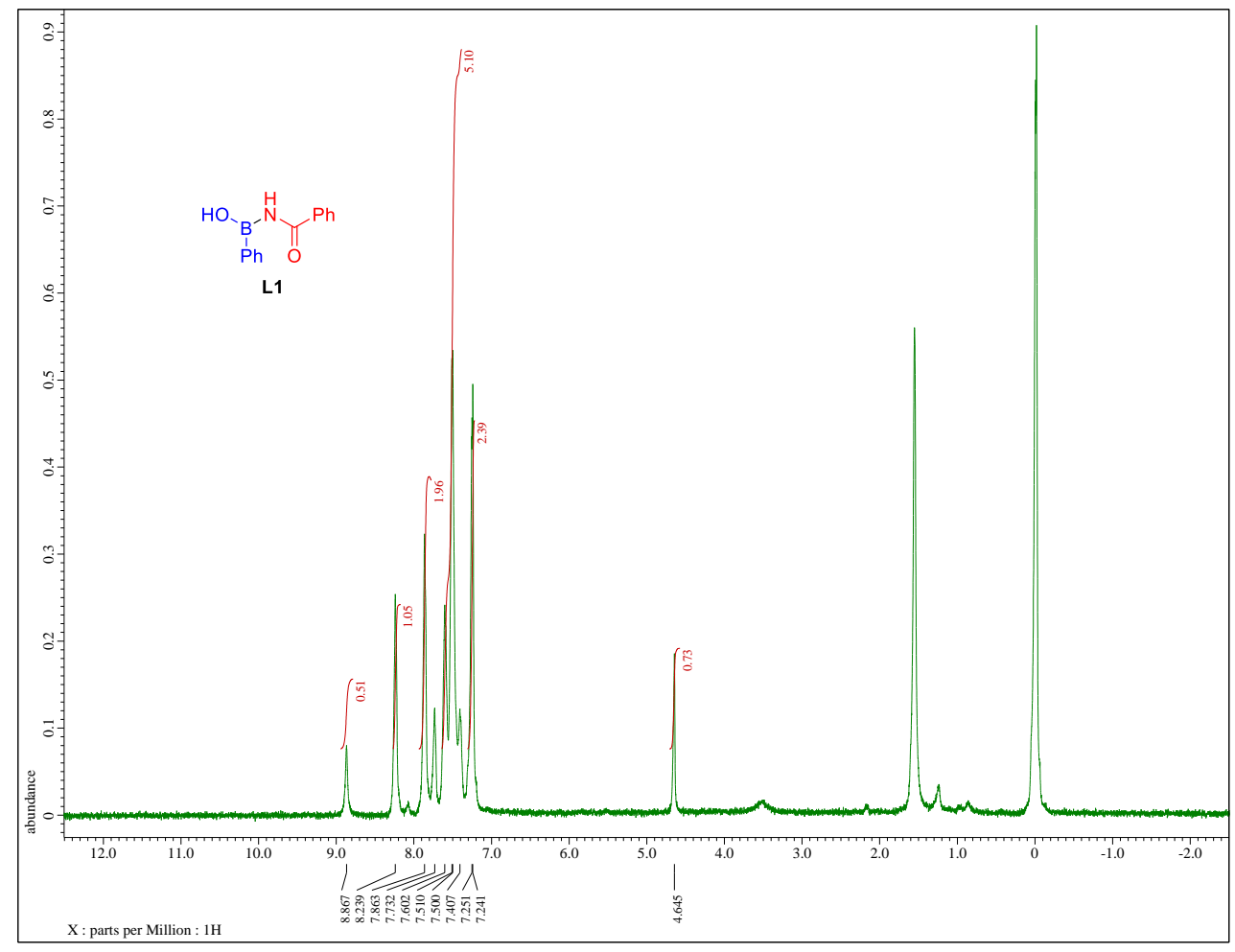

Figure S70-1. ${ }^{1} \mathrm{H}$ NMR spectrum of compound $\mathbf{L 1}\left(500 \mathrm{MHz}, \mathrm{CDCl}_{3}\right)$

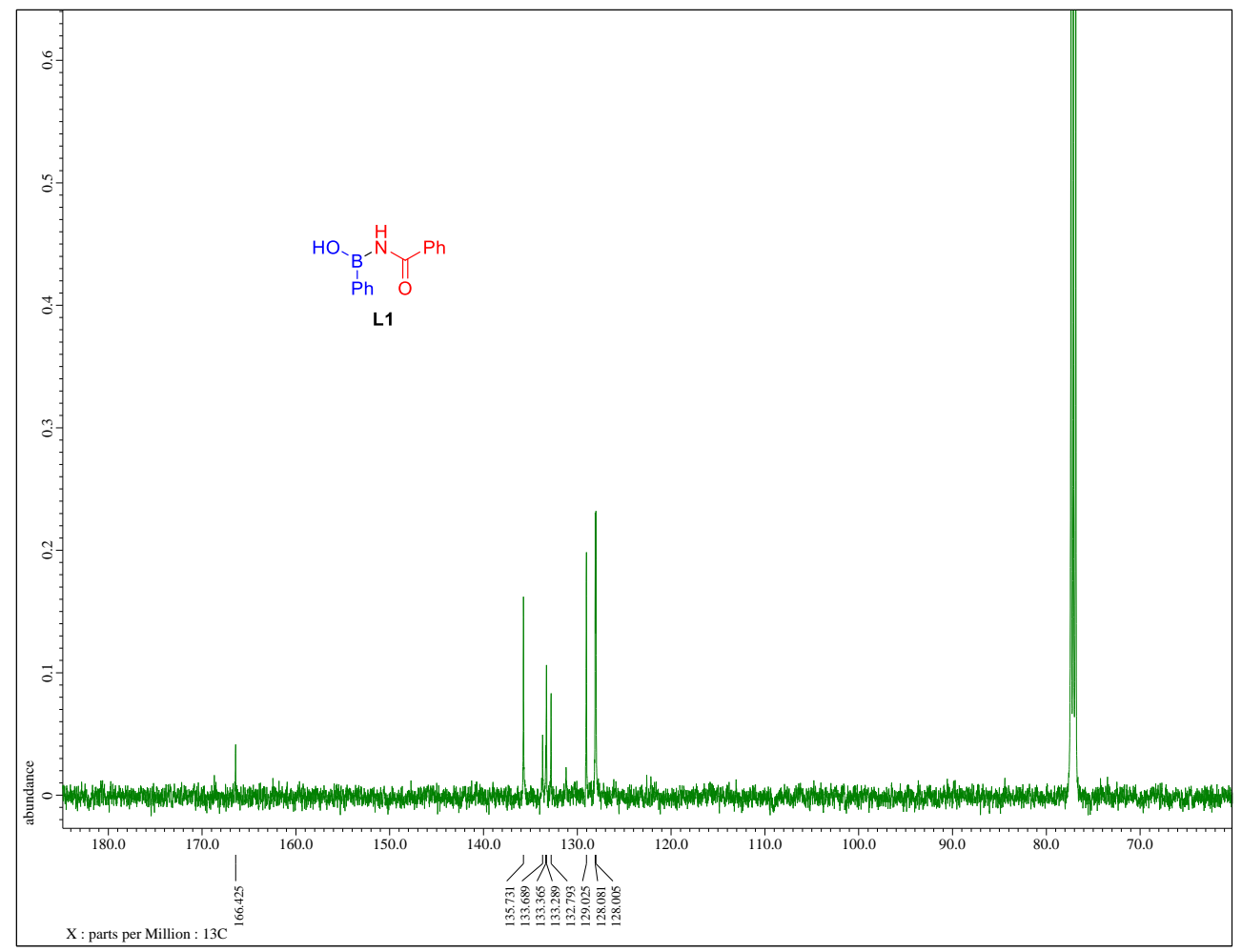

Figure S70-2. ${ }^{13} \mathrm{C}$ NMR spectrum of compound $\mathrm{L} 1\left(125 \mathrm{MHz}, \mathrm{CDCl}_{3}\right)$ 


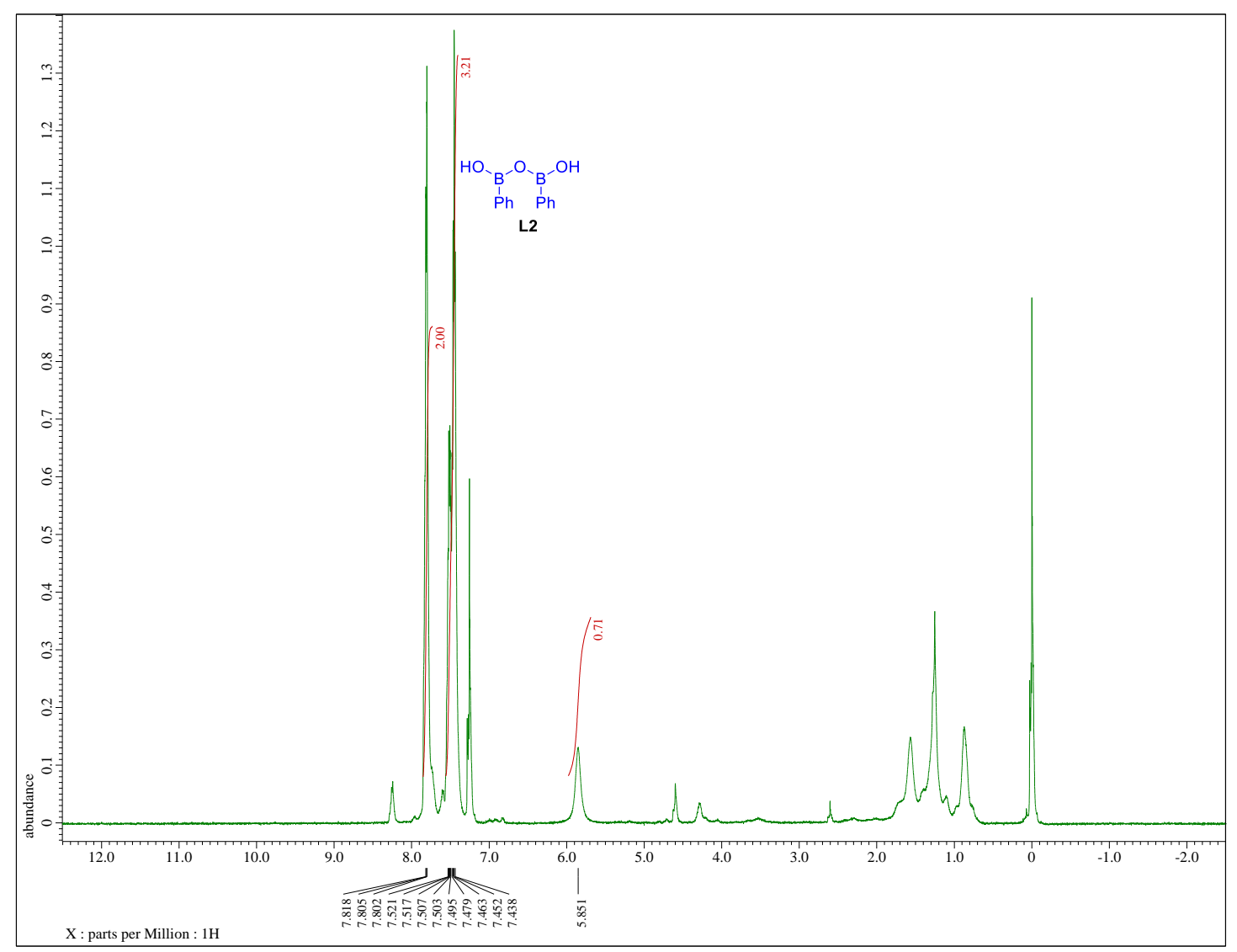

Figure S71-1. ${ }^{1} \mathrm{H}$ NMR spectrum of compound $\mathbf{L 2}\left(500 \mathrm{MHz}, \mathrm{CDCl}_{3}\right)$

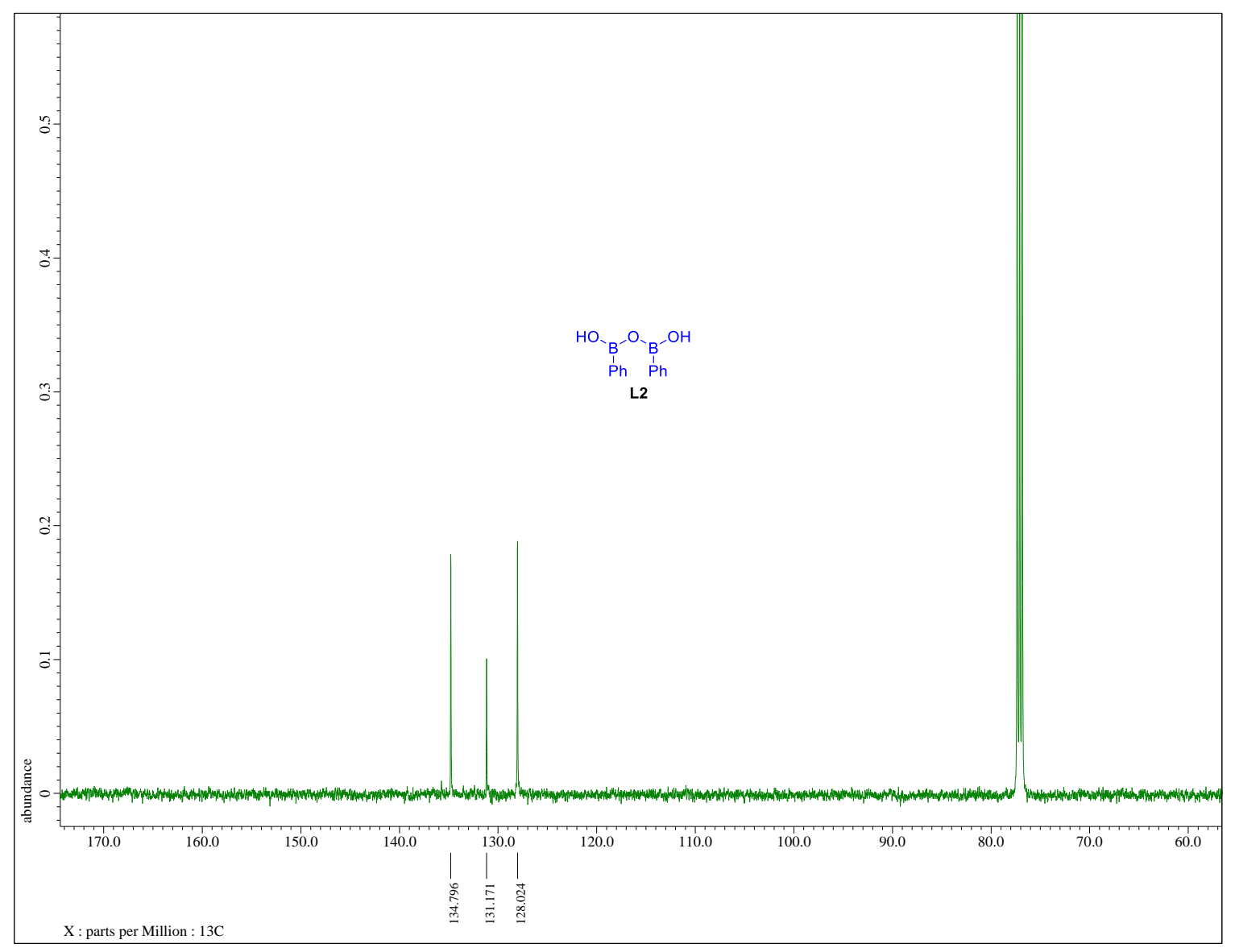

Figure S71-2. ${ }^{13} \mathrm{C}$ NMR spectrum of compound $\mathbf{L} 2\left(125 \mathrm{MHz}, \mathrm{CDCl}_{3}\right)$ 


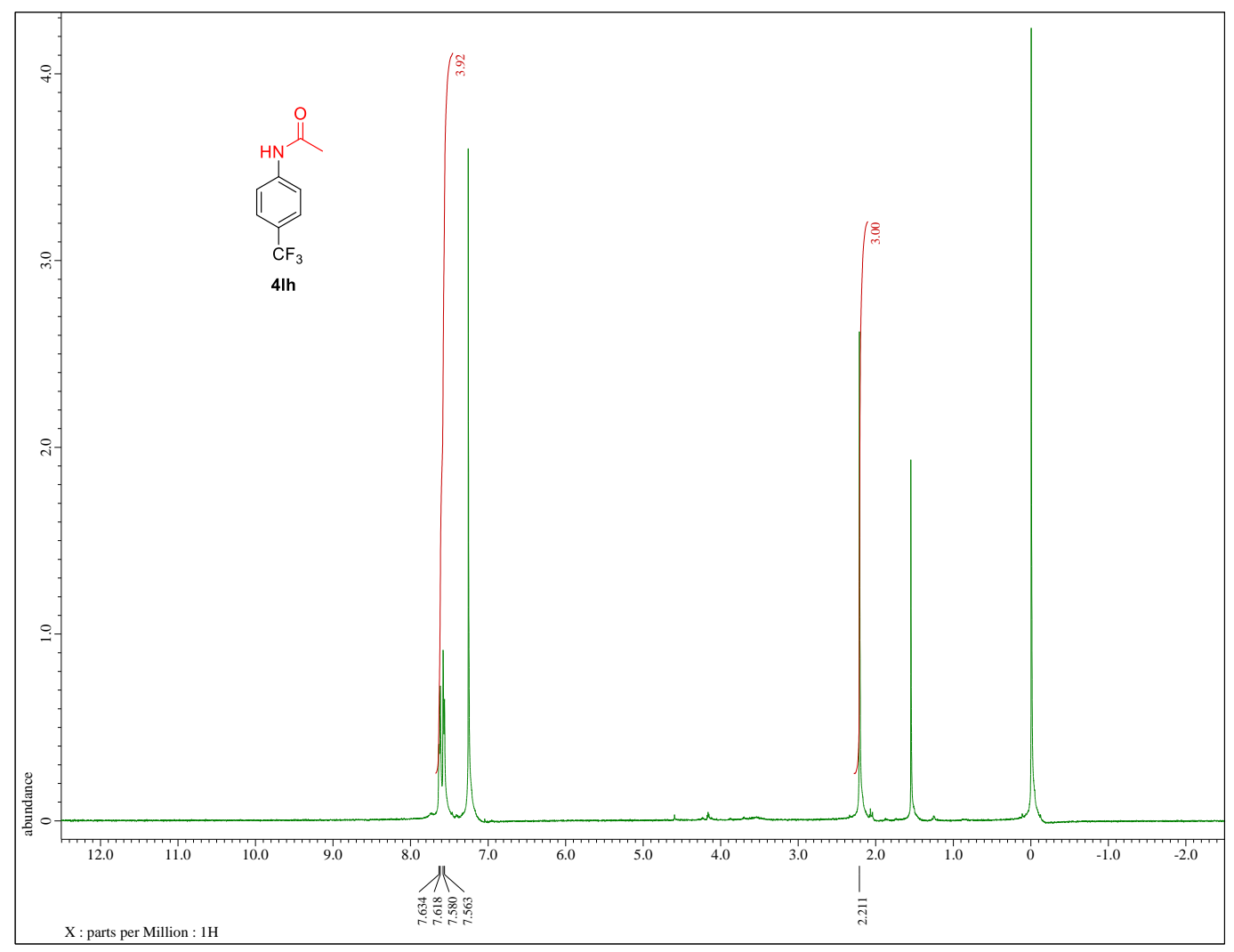

Figure S72-1. ${ }^{1} \mathrm{H}$ NMR spectrum of compound $4 \mathrm{lh}\left(500 \mathrm{MHz}, \mathrm{CDCl}_{3}\right)$

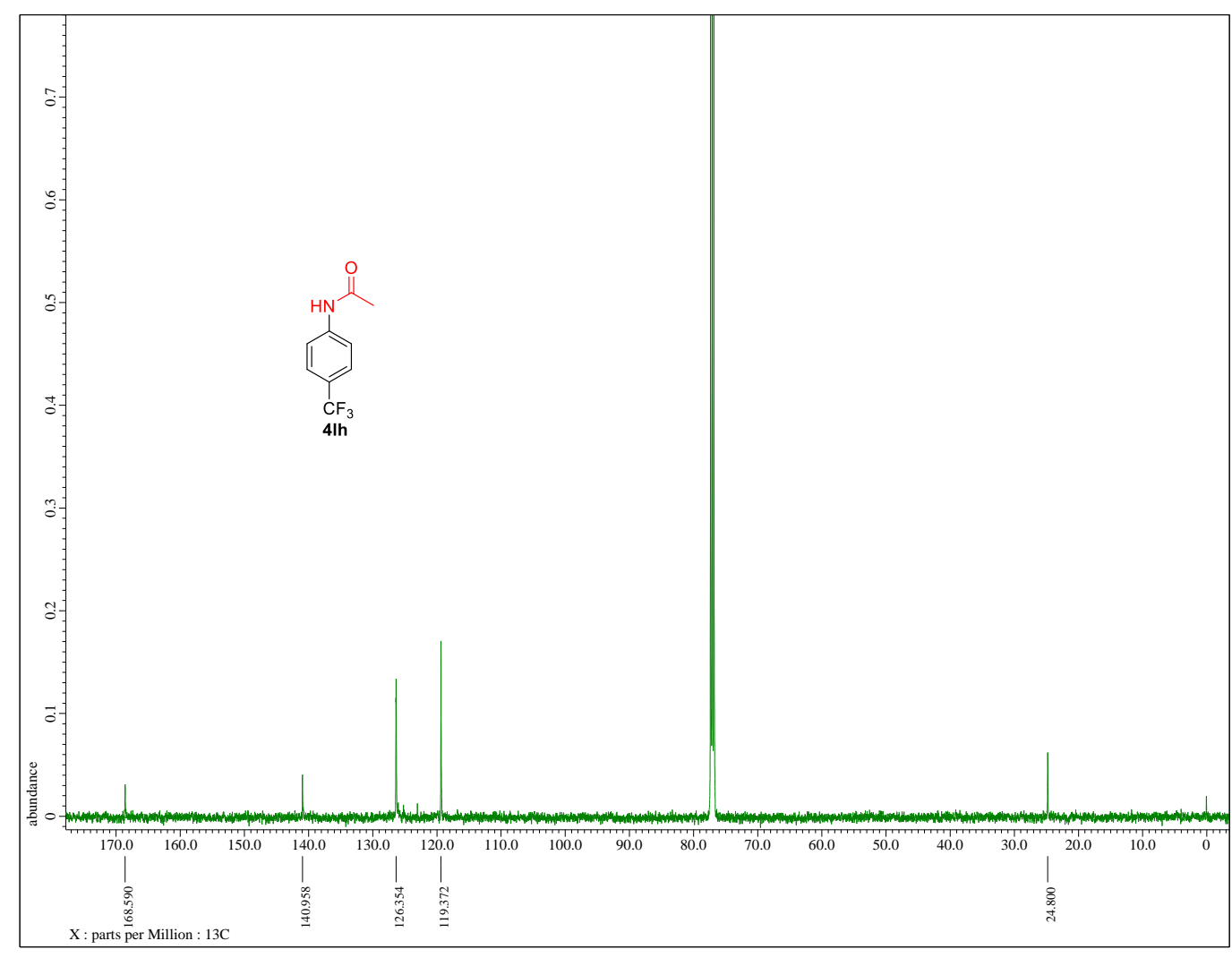

Figure S72-2. ${ }^{13} \mathrm{C}$ NMR spectrum of compound 4 lh $\left(125 \mathrm{MHz}, \mathrm{CDCl}_{3}\right)$ 


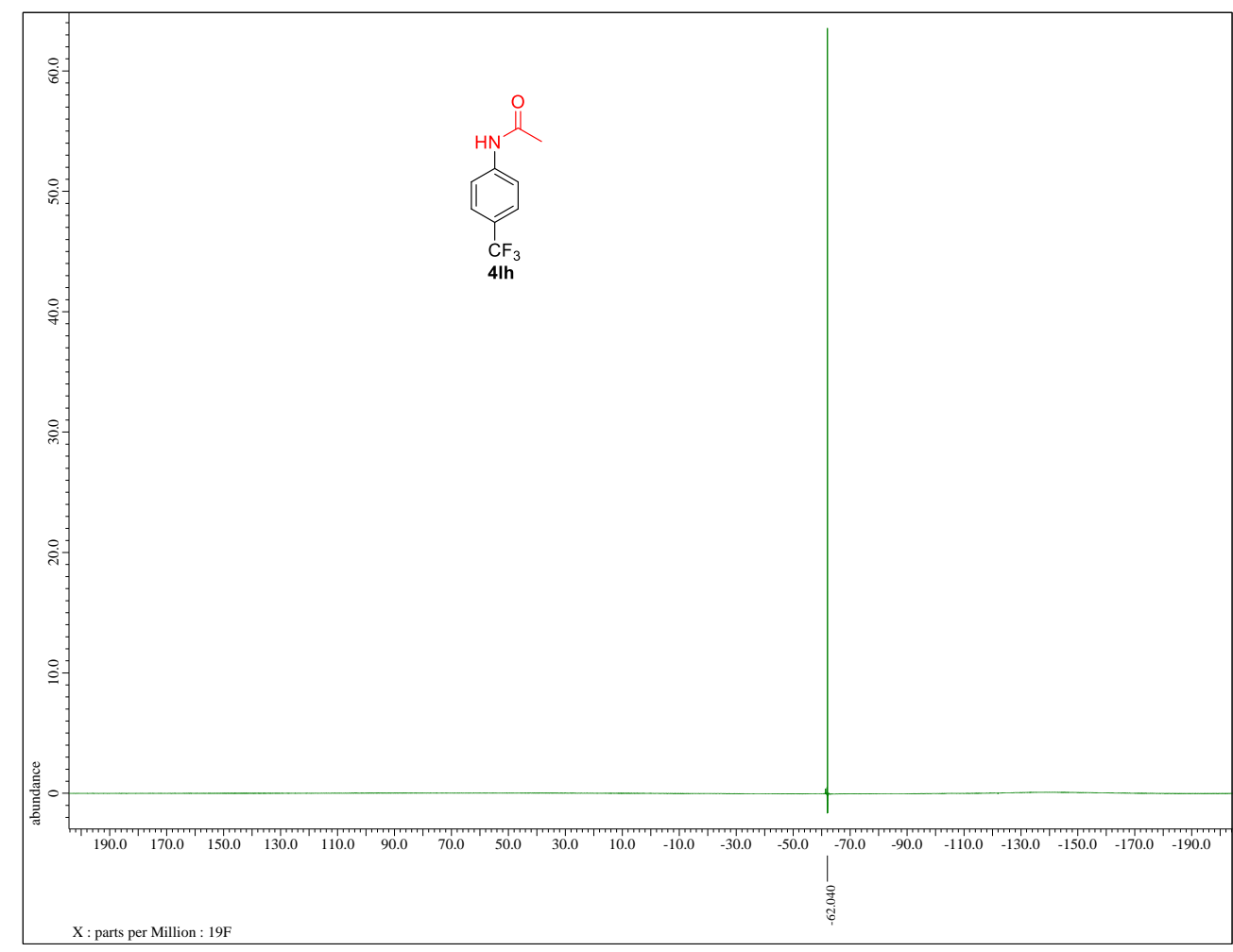

Figure S72-3. ${ }^{19} \mathrm{~F}$ NMR spectrum of compound 4lh $\left(470 \mathrm{MHz}, \mathrm{CDCl}_{3}\right)$ 


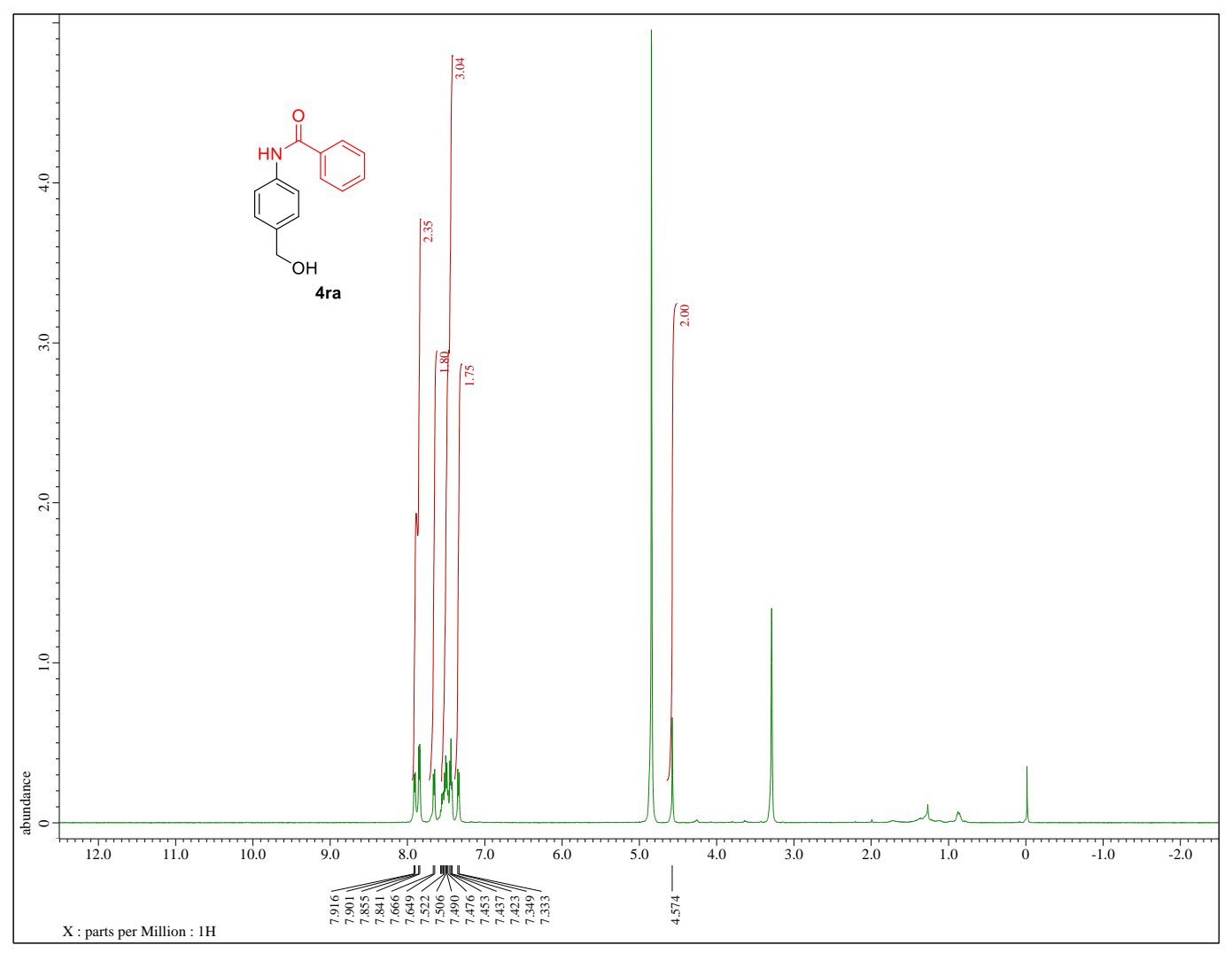

Figure S73-1. ${ }^{1} \mathrm{H}$ NMR spectrum of compound 4ra (500 MHz, MeOD)

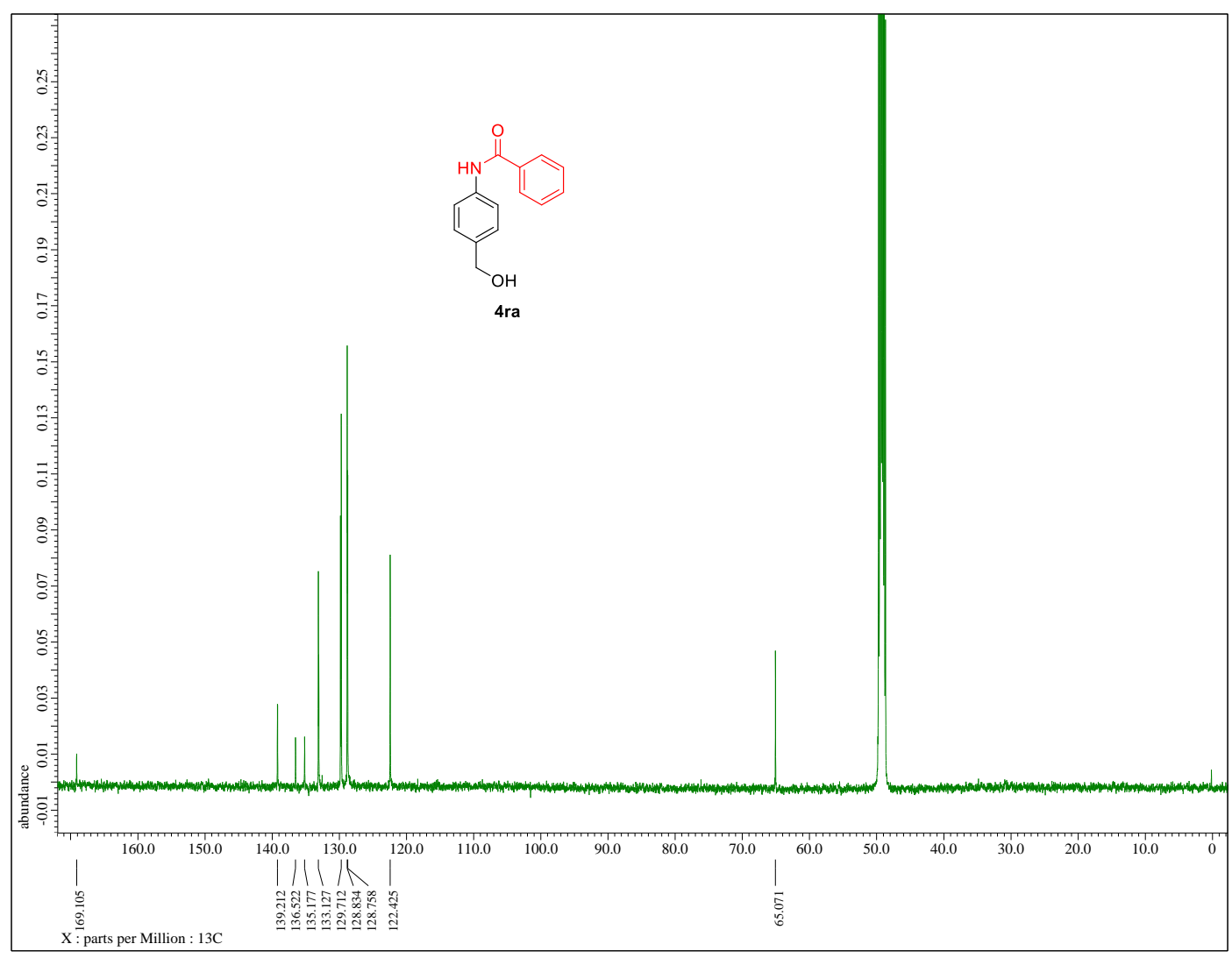

Figure S73-2. ${ }^{13} \mathrm{C}$ NMR spectrum of compound 4ra (125 MHz, MeOD) 


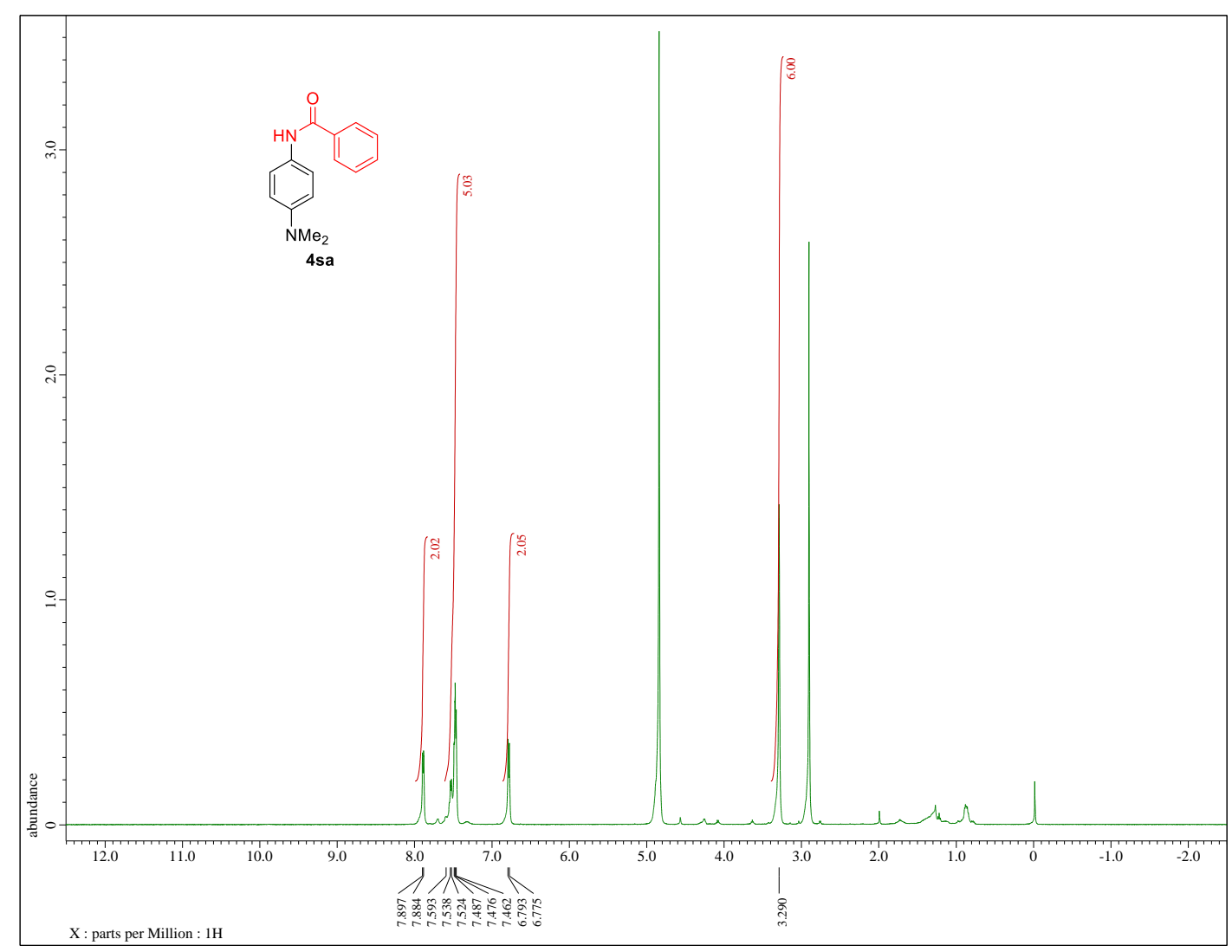

Figure S74-1. ${ }^{1} \mathrm{H}$ NMR spectrum of compound 4sa (500 MHz, MeOD)

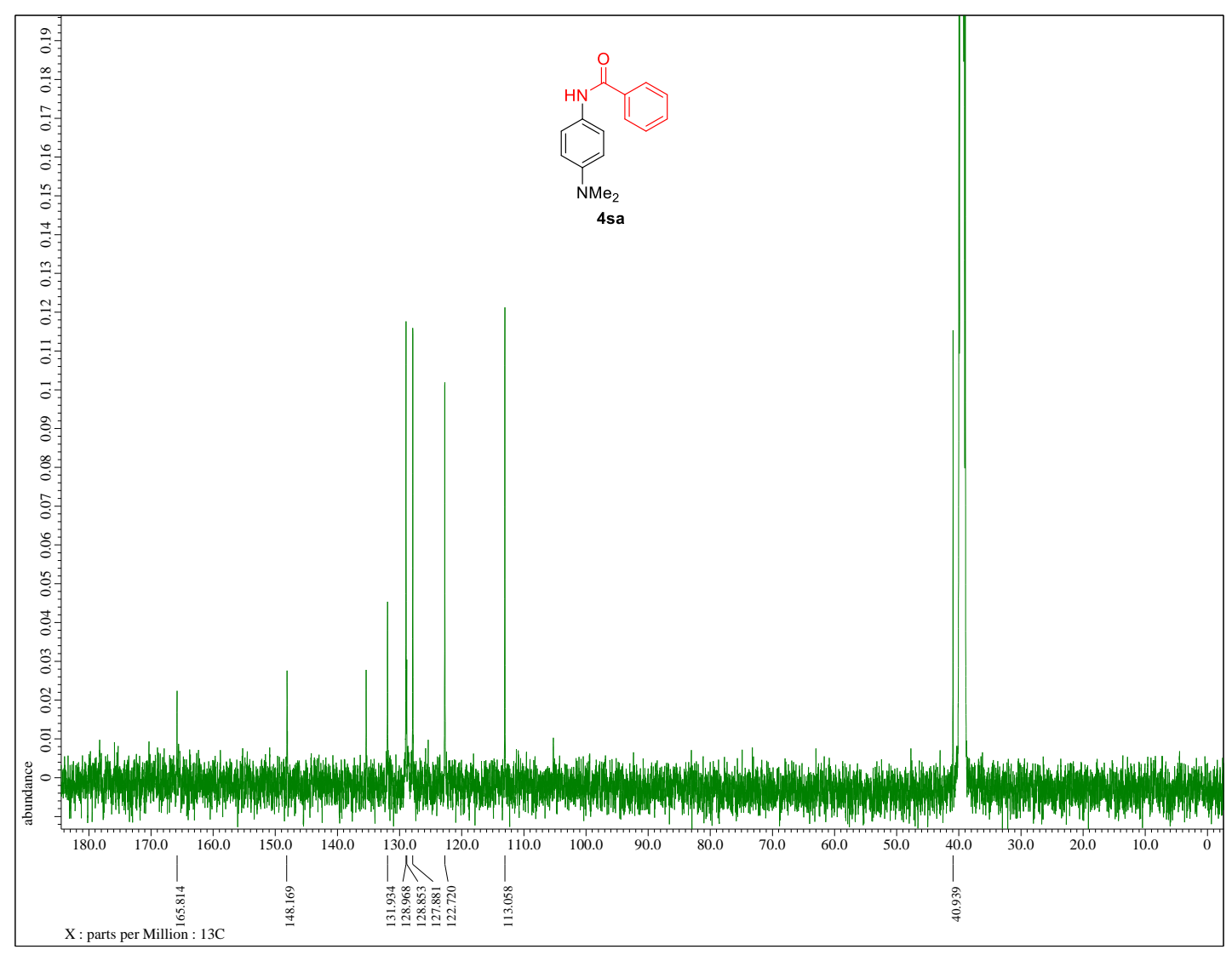

Figure S74-2. ${ }^{13} \mathrm{C}$ NMR spectrum of compound 4sa (125 MHz, DMSO- $\left.d_{6}\right)$ 